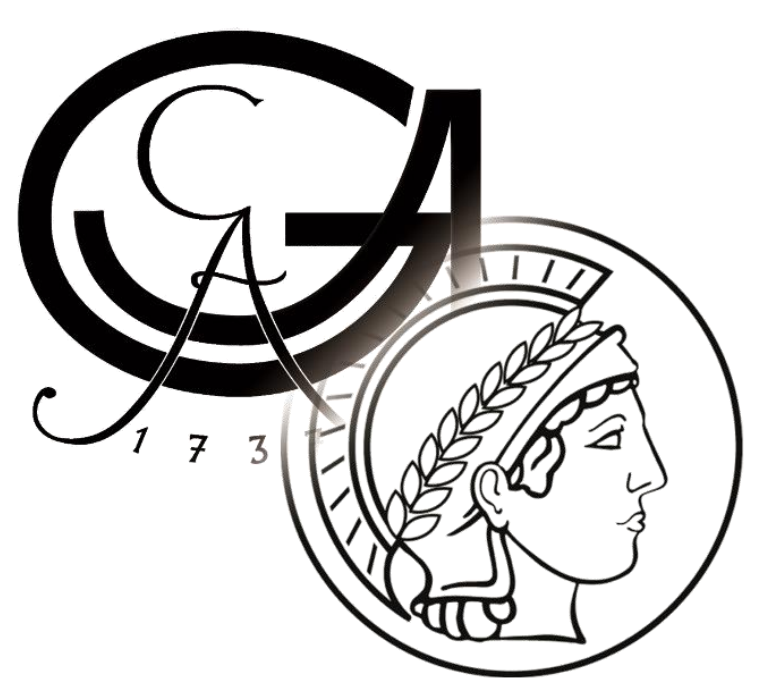

\title{
On the molecular basis of $\alpha$-synuclein aggregation on phospholipid membranes in the presence and absence of anle138b
}

\author{
Dissertation \\ for the award of the degree \\ "Doctor rerum naturalium" \\ of the Georg-August-Universität Göttingen \\ within the doctoral program \\ Biomolecules: Structure - Function - Dynamics \\ of the Georg-August University School of Science (GAUSS)
}

Submitted by

Leif Antonschmidt

from

Oldenburg

Göttingen, 2019 


\section{Thesis Committee}

Prof. Dr. Christian Griesinger

(Max-Planck-Institute for Biophysical Chemistry, Göttingen)

Prof. Dr. Claudia Steinem

(Institute of Organic and Biomolecular Chemistry, Göttingen)

Prof. Dr. Bert De Groot

(Max-Planck-Institute for Biophysical Chemistry, Göttingen)

\section{Members of the Examination Board}

Referee: Prof. Dr. Christian Griesinger

$2^{\text {nd }}$ Referee: Prof. Dr. Claudia Steinem

\section{Further members of the Examination Board}

Prof. Dr. Bert De Groot

(Max-Planck-Institute for Biophysical Chemistry, Göttingen)

Prof. Dr. Marina Bennati

(Max-Planck-Institute for Biophysical Chemistry, Göttingen)

Prof Dr. Markus Zweckstetter

(German Center for Neurodegenerative Diseases, Göttingen)

Prof. Dr. Henning Urlaub

(Max-Planck-Institute for Biophysical Chemistry, Göttingen)

Date of oral examination: November 28, 2019 


\section{Acknowledgement}

First of all, I'd like to thank Prof. Dr. Griesinger for the very interesting topic and the possibility to work under his supervision. Your door has always been open and I thank you for the constant interest in my work. I am forever grateful to you for giving me the opportunity to expand my knowledge abroad as a visiting scientist.

I'd like to express my gratitude to the members of my thesis committee, Prof. Dr. Steinem and Prof. De Groot, for the fruitful discussions and valuable scientific advice. The collaborations resulting from this interaction have proven invaluable to the progress of the project.

Special thanks go to Dr. Stefan Becker, Karin Giller and Melanie Wegstroth for investing countless hours into preparing high quality protein samples for me.

I'd like to thank my dear colleagues Kris Runge, Dr. Loren Andreas and Dr. Riza Dervişoğlu for the productive collaboration on this project. Without your work, this thesis wouldn't be what it is.

Further l'd like to thank Dirk Matthes for his remarkable work on MD-simulation, greatly contributing to this project.

I'd like to thank Prof. Dr. Ratnesh Lal for giving me the opportunity to work with his group at the University of California, San Diego. Working with your group has been inspiring.

Special thanks go to Dr. Joon Lee for teaching me the art of Atomic Force Microscopy and Dr. Christian Schmidt for teaching me the use of NMR spectrometers.

I'd like to thank Prof. Dr. Steinem and Prof. Dr. Janshoff for letting me use their lab space and instruments. In addition, l'd like to thank Ingo Mey for helpful discussions and technical support in AFM-experiments.

In addition, I'd like to thank Kuan-Ting Pan and Prof. Dr. Henning Urlaub for their collaboration in photoaffinity studies.

Further thanks go to Gudrun Heim and Dietmar Riedel for measuring countless TEM micrographs.

I'd also like to thank my master student Simon Kohlmann and my interns Hannes Witt and Gabriel Jose Villamil for their help with experiments.

I'd like to particularly mention my utmost gratitude to Dr. Nasrollah "Hessam" Rezaei-Ghaleh for sharing his limitless knowledge with me. You are a truly inspiring person and I have enjoyed every conversation we had, be it scientific or philosophical. 
Of course, l'd like to thank all of my colleagues over, many of whom have become friends over the years. I'd like to specially mention Dirk, Sebastian T., Christian, Michele, Pablo, Niels, Adriana, Sebastian F., Supriya and Eibe.

Special thanks also go to Petra Breiner and Dr. Dirk Bockelmann for administrative work and help in any situation.

I'd also like to thank Dr. Christian Bartling and Vrinda Sant for proofreading this thesis and providing valuable scientific input.

Words fail me in expressing my gratitude to my loving fiancé Olga. I am truly lucky to have found such an amazing person to share my life with. Your unconditional support in every situation of my life has made all of this possible. I love you with all my heart.

This work is dedicated to my parents for the loving and unconditional support in all of my life choices. 
"Wir haben ganz unten alles erkundet, all die schmutzigen Ecken Kampfkunstschüler: Schlagen, Ducken, Einstecken.

Fallen, wieder aufstehen, stolpern, gehen, laufen.

Die schwersten Stufen waren rückblickend die ersten Stufen, schmutzige Stufen.

Heute glänzt es da unten, denn jedes Mal wenn wir gefallen sind blieb Staub in unseren Wunden, an Kleidung haften.

Das alles sind nur Teile einer dicken Haut - liebe den Staub. Lerne ihn lieben, er geht nie wieder raus - liebe deinen Staub. "

Mach One

"Neue Sonne" 


\section{Table of Contents}

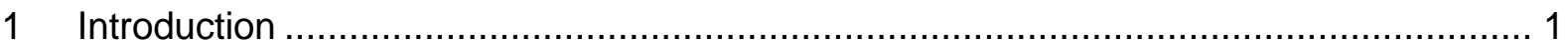

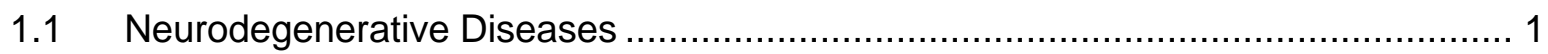

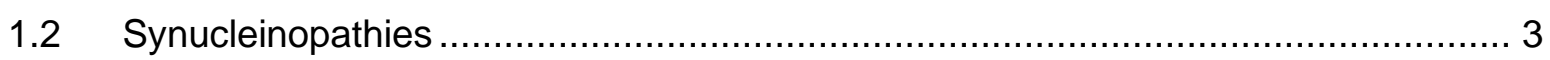

1.2.1 Pathologies, Clinical Symptoms and Treatment ...................................... 3

1.2.2 Etiology and pathogenesis ....................................................................... 5

1.2.3 The role of protein aggregation in pathogenesis ...................................... 6

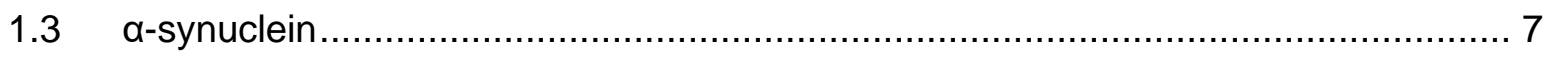

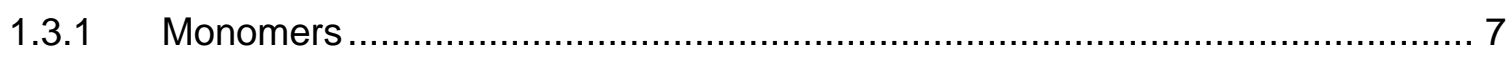

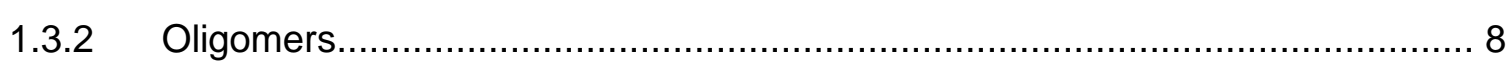

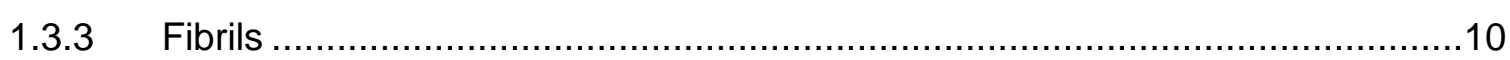

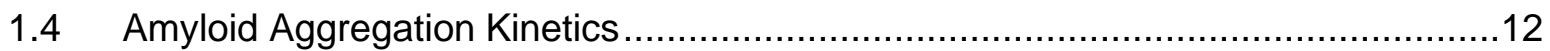

1.4.1 Interaction of $\alpha$-synuclein with phospholipid membranes ..............................12

1.4.2 Protein Misfolding Cyclic Amplification (PMCA) .........................................13

1.5 Anle138b as a potential disease modifying agent............................................15

1.6 Anle138b in Phospholipid Membranes ...........................................................16

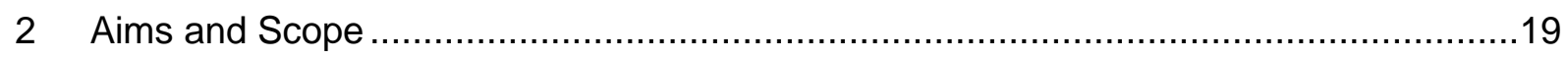

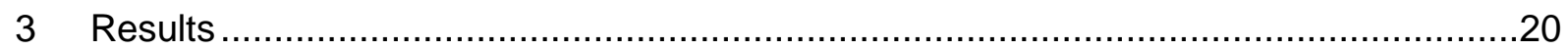

3.1 Oligomer formation in the presence of phospholipids by PMCA ….......................20

3.1.1 Kinetics of $\alpha S$ Aggregation under PMCA Conditions ...................................20

3.1.2 Quantification of monomeric aS binding to phospholipids.............................22

3.1.3 Evidence for $\alpha S$ oligomers and an Effort at Quantification ..............................23

3.1.4 Morphological Analysis of aS Oligomers by EM and AFM ............................28

3.1.5 Evolution of Secondary Structure during $\alpha S$ aggregation ...............................30

3.2 Characterization of $\alpha S$ fibrils and intermediates by sSNMR ...................................

3.2.1 Assignments of aS fibrils in the presence of phosplipids ..............................34

3.2.2 aS intermediates in the presence of phospholipids.....................................41

3.3 Interaction of anle138b with $\alpha S$ aggregates................................................. 45

3.3.1 Influence of anle138b on $\alpha$-synuclein aggregation kinetics ...........................45

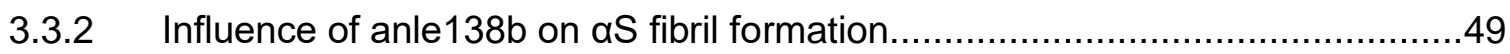

3.3.3 DNP-enhanced solid-state NMR experiments ..........................................51

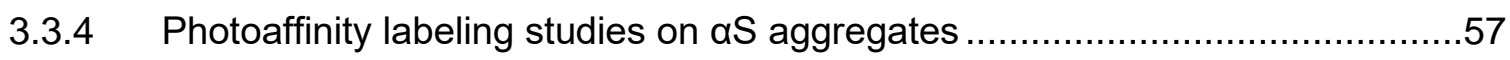

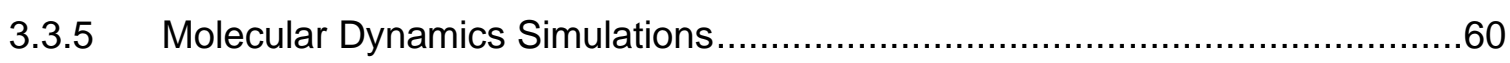

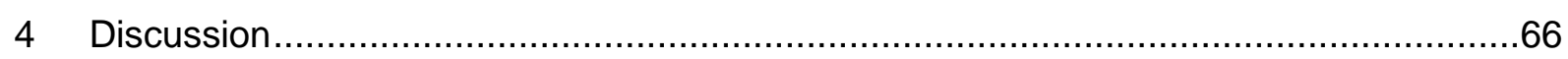

4.1 PMCA drives the formation of membrane bound $\alpha \mathrm{S}$ oligomers ...........................66

4.2 A new aS fibril polymorph or just Polymorph $2 \mathrm{c}$ ? ......................................... 70 
4.3 Structure of $\alpha S$ intermediates and insights into nucleation ................................72

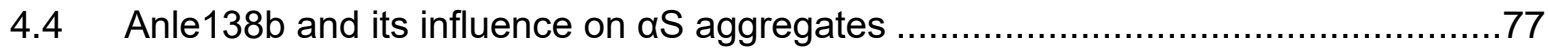

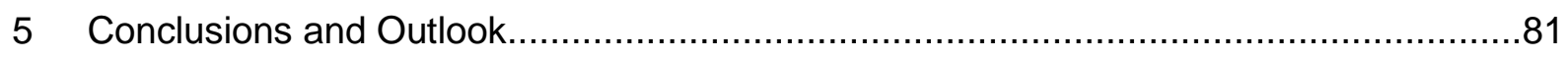

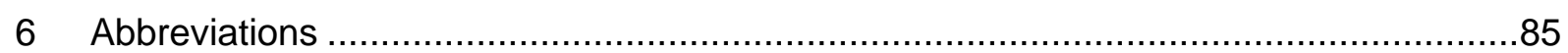

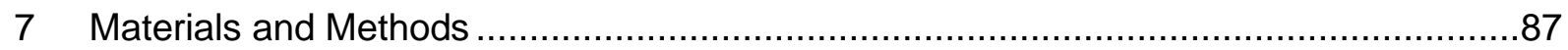

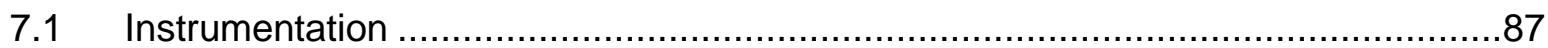

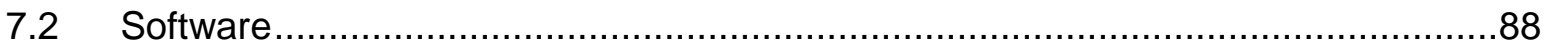

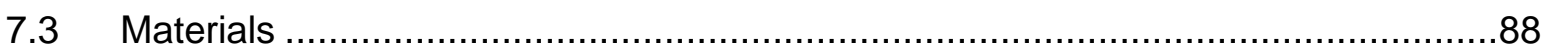

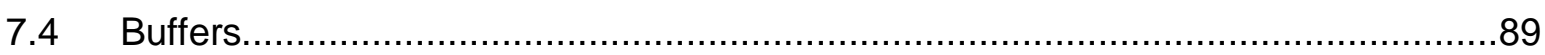

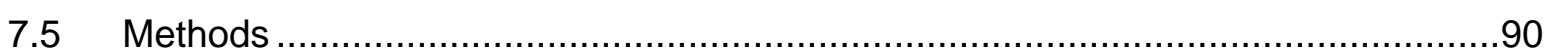

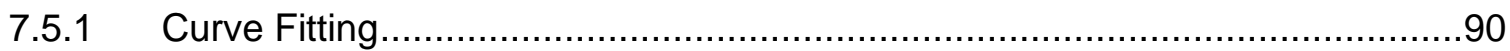

7.5.2 Preparation of Small Unilamellar Vesicles (SUVs) .......................................90

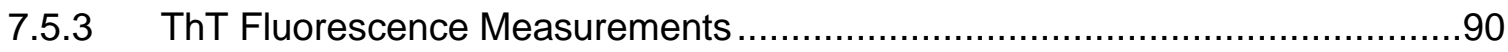

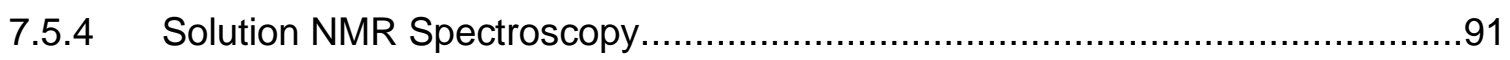

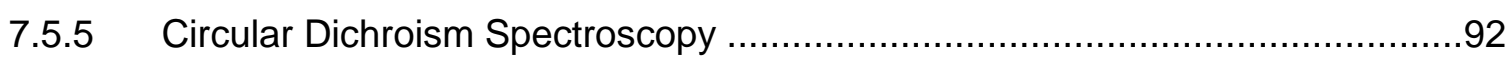

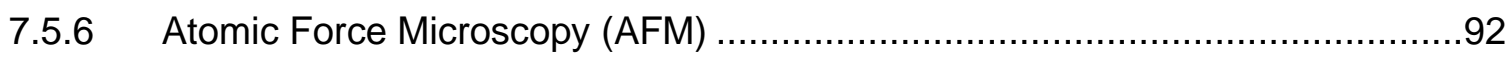

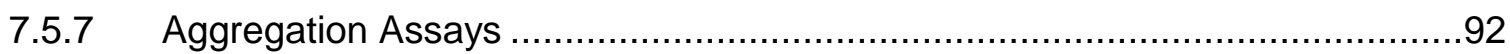

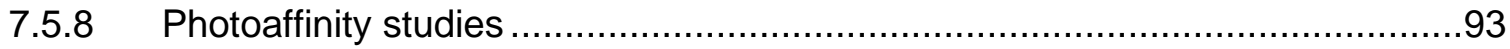

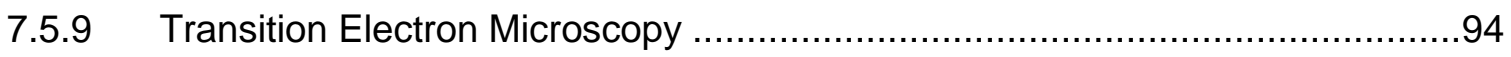

7.5.10 Estimation of oligomer concentration from kinetic data................................94

7.5.11 Secondary Structure Calculation by BeStSel.............................................96

7.5 .12 ssNMR

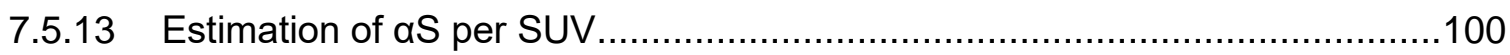

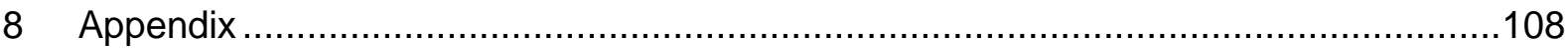





\section{Introduction}

\subsection{Neurodegenerative Diseases}

To date, neurodegenerative diseases (NDDs) remain one of the major challenges in academic as well as pharmaceutical research, especially in the light of growing life expectancies. ${ }^{[1,2,3]}$ Neurodegenerative diseases is an umbrella term ${ }^{[4]}$ for a broad array of conditions, which are characterized by the functional loss and death of neurons within the central nervous system (CNS) such as Alzheimer's Disease (AD), Parkinson's Disease (PD), Multiple System Atrophy (MSA), Amyotrophic Lateral Sclerosis (ALS), Frontotemporal Dementia (FTD) and Creutzfeldt-Jakob Disease (CJD). ${ }^{[5]}$ Other common features of these diseases are abnormalities in protein degradation, mitochondrial dysfunction and aberrant misfolding of proteins, ultimately leading to deposits in neurons and other cells and compartments. ${ }^{[6]}$ In many NDDs it is not entirely clear, to which extent genetic and environmental factors contribute to triggering these diseases. ${ }^{[7]}$ Treatment, so far, focuses mainly on the alleviation of symptoms (symptomatic treatment) rather than curing the underlying disease (disease modifying treatment). ${ }^{[8]}$ Crucially the development of therapeutic agents is often hampered by a poor understanding of disease mechanisms and promising therapies often fail in late stages of clinical trials. ${ }^{[9]}$ This problem is further aggravated by the lack of means for early and accurate diagnosis of patients and proper classification in clinical trials, although significant efforts have been made to alleviate these problems. ${ }^{[10]}$

\begin{tabular}{|c|c|c|c|c|}
\hline Disease & Microscopic lesion & Location & Aggregated protein & Figure 1.1.1: \\
\hline \multirow[t]{3}{*}{ Alzheimer's disease } & Amyloid plaque & Extracellular & Amyloid- $\beta(\mathrm{A} \beta)$ & \multirow{12}{*}{$\begin{array}{l}\text { Common neuro- } \\
\text { degenerative } \\
\text { diseases and the } \\
\text { associated } \\
\text { proteins leading } \\
\text { to proteinaceous } \\
\text { inclusion } \\
\text { bodies. }{ }^{[11]}\end{array}$} \\
\hline & Neurofibrillary tangle & Intracytoplasmic (neurons) & Tau & \\
\hline & $\begin{array}{l}\text { Lewy bodies (seen in } \\
\text { Lewy body variant) }\end{array}$ & Intracytoplasmic (neurons) & $\alpha$-synuclein & \\
\hline $\begin{array}{l}\text { Amyotrophic lateral } \\
\text { sclerosis }\end{array}$ & Hyaline inclusions & Intracytoplasmic (neurons) & Superoxide dismutase-1 (SOD1) & \\
\hline $\begin{array}{l}\text { Cortical basal } \\
\text { degeneration/ } \\
\text { progressive } \\
\text { supranuclear palsy } \\
\end{array}$ & Tau positive inclusions & $\begin{array}{l}\text { Intracytoplasmic (neurons, } \\
\text { oligodendroglia and astrocyes) }\end{array}$ & Tau & \\
\hline $\begin{array}{l}\text { Dementia with Lewy } \\
\text { bodies }\end{array}$ & Lewy bodies & Intracytoplasmic (neurons) & $\alpha$-synuclein & \\
\hline Huntington disease & Neuronal inclusions & Intranuclear (neurons) & $\begin{array}{l}\text { Huntington (containing } \\
\text { polyglutamine repeat expansion) }\end{array}$ & \\
\hline $\begin{array}{l}\text { Multiple system } \\
\text { atrophy }\end{array}$ & $\begin{array}{l}\text { Glial cytoplasmic } \\
\text { inclusions }\end{array}$ & Intracytoplasmic (oligodendroglia) & $\alpha$-synuclein & \\
\hline Parkinson's disease & Lewy bodies & Intracytoplasmic (neurons) & $\alpha$-synuclein & \\
\hline Pick's disease & Pick bodies & Intracytoplasmic (neurons) & Tau & \\
\hline Prion diseases & Prion plaques & Extracellular & $\begin{array}{l}\text { Protease-resistant prion protein } \\
(\operatorname{PrP})\end{array}$ & \\
\hline Spinocerebellar ataxia & Neuronal inclusions & Intranuclear (neurons) & $\begin{array}{l}\text { Ataxin (containing polyglutamine } \\
\text { repeat expansion) }\end{array}$ & \\
\hline
\end{tabular}


Moreover, in many disorders single molecular targets or pathways have yet to be identified, which might lead to success in drug discovery. ${ }^{[8]}$ Growing evidence hints to protein aggregation taking a key role in pathological progression ${ }^{[12,13]}$, although this observation is not entirely free of controversy. ${ }^{[14]}$ The native proteins involved in the pathogenesis of NDDs are usually depicted as completely unstructured, although this is often an oversimplified picture. ${ }^{[15]}$ Deviation from normal functionality of these proteins in most of the diseases leads to small oligomers or larger clusters of protein, which are of different specific toxicity. ${ }^{[16]}$ Figure 1.1.1 shows a selection of aggregation prone proteins along with the location of the protein deposits formed through their misfolding. ${ }^{[11]}$ Ultimately, these proteins form so-called amyloids, proteinaceous assemblies that are characterized by their cross- $\beta$ quaternary structure and can be found inside the hallmark inclusion bodies of the corresponding disease. ${ }^{[13]}$

The pathways leading to aggregation offer potential targets for the development of new therapeutic agents as shown in Figure 1.1.2. Even though a lot of different proteins are associated with a large variety of diseases, structure and formation of the aggregates are often very similar. ${ }^{[17]}$ Since the structural heterogeneity of the disease relevant species impedes rational drug design, many pharmaceutical companies have exited the field of NDD drug development. ${ }^{[18,19]}$ In this light the importance of academic research has often been pointed out. ${ }^{[20]}$

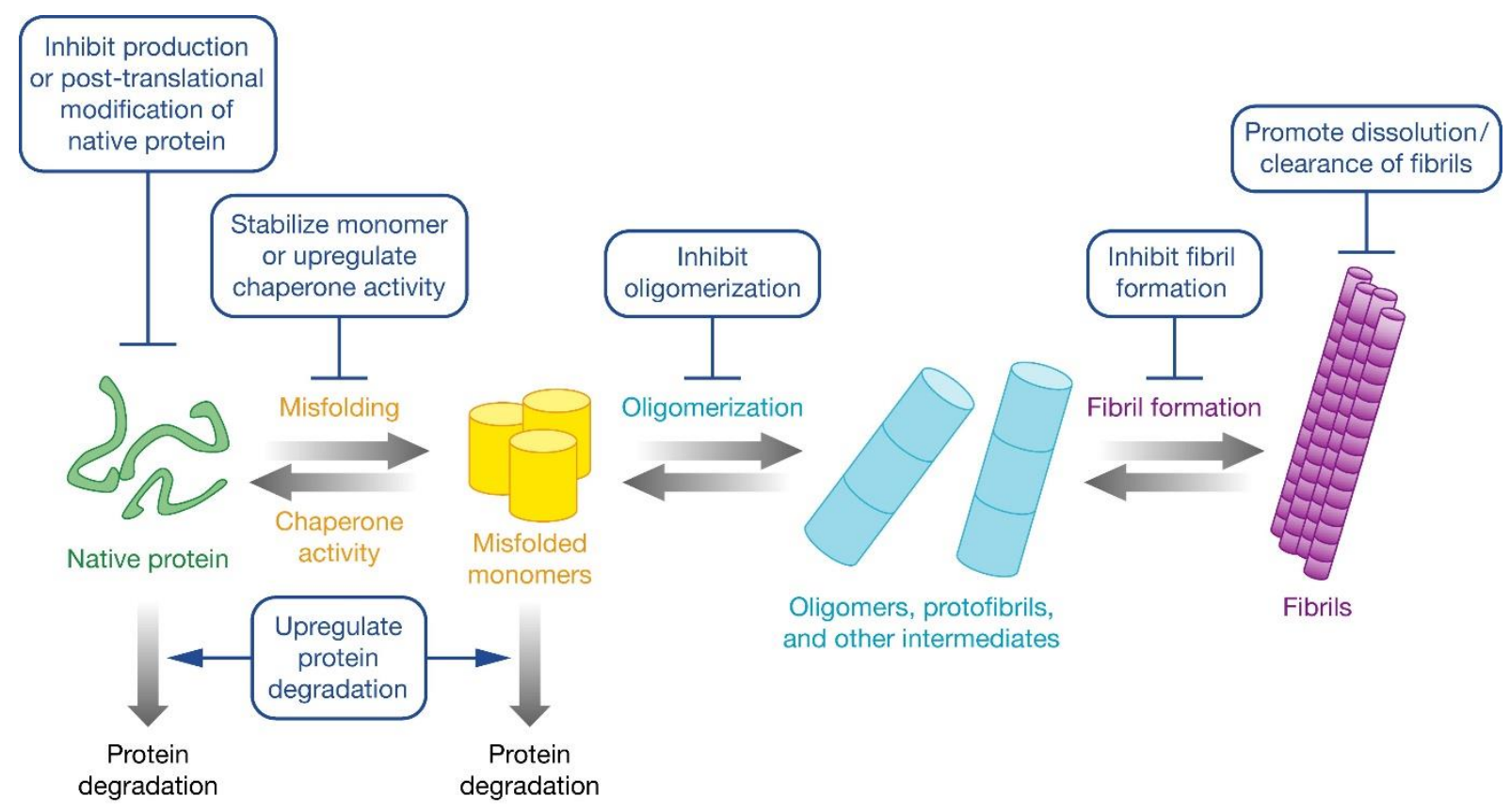

Figure 1.1.2: Protein aggregation pathways in NDDs and possible intervention points for therapeutic agents. 


\subsection{Synucleinopathies}

The neurodegenerative diseases displaying pathology along with an aberrant accumulation of misfolded $\alpha$-synuclein ( $\alpha S$ ) in neurons and glial cells are called synucleinopathies. This family of diseases encompasses mainly Parkinson's Disease (PD), Dementia with Lewy Bodies (DLB), and Multiple System atrophy (MSA). ${ }^{[1]}$

\subsubsection{Pathologies, Clinical Symptoms and Treatment}

Parkinson's Disease (PD) was first described by James Parkinson in 1817 and is the most prevalent among synucleopathies and the second most common neurodegenerative disorder affecting the brain. ${ }^{[22]}$ PD begins in mid to late life and common symptoms include motor impairment, autonomic dysfunction and often psychological and cognitive changes. ${ }^{[23]}$ The four main symptoms found in PD patients are bradykinesia along with resting tremor, rigidity and postural instability. ${ }^{[24]}$ However, not all of these symptoms are found in patients to the same extent and many more symptoms, especially nonmotor features, pose viable measures for early diagnosis. ${ }^{[25]}$ In general, PD itself is not fatal, however complications arising from symptoms can lead to death. ${ }^{[26]}$ The pathophysiological hallmarks of PD are the loss of dopaminergic neurons in the substantia nigra pars compacta and the presence of inclusion bodies, known as Lewy Bodies (LB) and Lewy Neurites (LN), consisting of aggregated $\alpha$ synuclein, alongside disrupted cell organelles and lipid membranes. ${ }^{27,28,29]}$

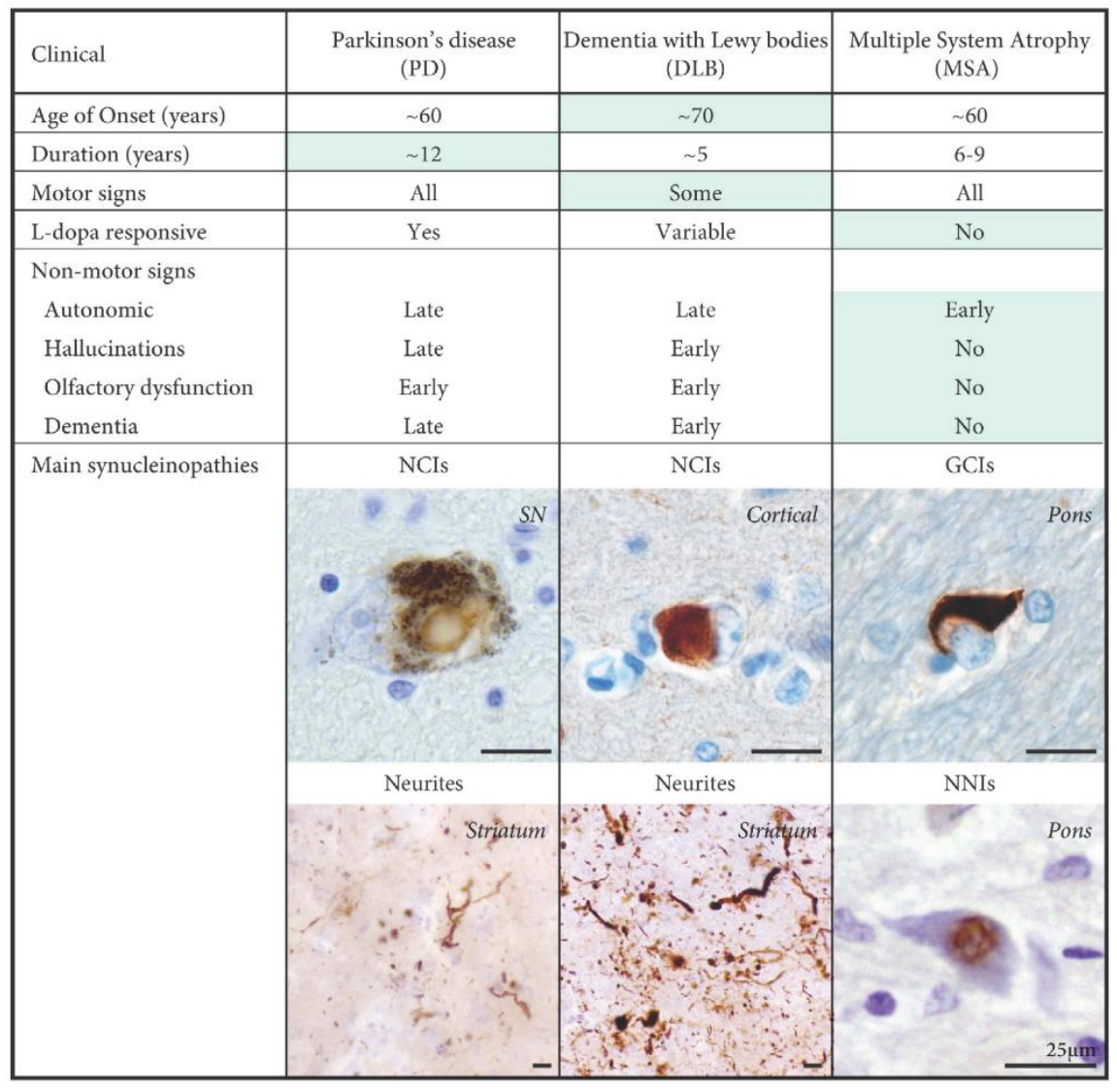

Figure 1.2.1:

Pathologies, symptoms and disease metrics shown in the common synucleinopathies.

Images show stain brain tissue of $\alpha$ synuclein inclusion bodies. ${ }^{[27]}$ 
Dementia with Lewy Bodies (DLB) is the second most common form of neurodegenerative dementia, accounting for up to $24 \%$ of the cases and affecting about $0.7 \%$ of the word population. ${ }^{[30]}$ DLB and PD share the occurrence of Lewy Bodies, but DLB shows a higher tendency towards cortical amyloid- $\beta(A \beta)$ plaques and neurofibrillary tangles much like in $A D$, albeit with less intensity. ${ }^{[31]}$ Patients suffering from DLB show a fluctuation in cognition, attention and alertness, alongside visual hallucinations and Parkinsonism. ${ }^{[31]} \mathrm{A}$ distinction between DLB and PD is made based on the onset of dementia in within the first year of the disease. If dementia occurs later patients are classified as Parkinson's Disease with Dementia (PDD). ${ }^{[32]}$ The substantial overlap in both neuropathological and clinical features of DLB and PDD make a distinction extremely challenging and calls for further research. ${ }^{\text {[3] }}$

MSA usually is diagnosed around the same age as PD, but is significantly more aggressive, with an average life expectancy from diagnosis of about 6-9 years (Figure 1.2.1). ${ }^{[27]}$ The histopathological hallmark of MSA are the so called glial cytoplasmic inclusions (GCls) located in the cytoplasm of oligodendrocytes consisting of $\alpha$-synuclein aggregates. ${ }^{[34]}$ These inclusions are specific to MSA, usually not occurring for PD or DLB. There are two major phenotypes of MSA, which are categorized by their predominant motor features: MSA-P (Parkinsonian subtype) and MSA-C (cerebellar subtype). The MSA-P variant shows features reminiscent of $P D$, such as bradykinesia, rigidity, tremor and postural instability. In MSA-C cerebellar ataxia is the main motor feature, often combined with speech and limb ataxia and cerebellar oculomotor dysfunction. ${ }^{[35,36]}$ Since various symptoms are shared between the respective diseases, PD and MSA are often misdiagnosed. ${ }^{[37]}$

To date, treatment of $\mathrm{PD}^{[38]}$, $\mathrm{DLB}^{[39]}$ as well as $\mathrm{MSA}^{[40]}$, is mainly symptomatic, due to the absence of any cure or disease modifying treatment. In PD, the most potent therapy is the administration of levodopa (L-dopa), which is converted by the body into dopamine and hence greatly ameliorates motor symptoms. However, ultimately all dopaminergic neurons die and treatment with L-dopa becomes ineffective. ${ }^{[41]}$ Another common treatment used for patients that no longer respond to pharmacological treatment is the so called deep brain stimulation (DBS). In this surgical procedure a small medical device, generating electrical impulses, is inserted into specific brain region. The electrical impulses then block abnormal electrical signals, thus alleviating PD motor symptoms. ${ }^{[42]}$ The exact mechanism of DBS is unclear and lack of technological evolution still limits its efficacy. ${ }^{[43]}$ For both DLB and MSA, treatment of parkinsonian symptoms usually follows similar protocols like in $\mathrm{PD}^{[39]}$, however response of MSA patients is generally poor and short-lived, making it a good marker for distinguishing MSA-P from PD. ${ }^{[44]}$ DLB patients show a cholinergic deficit, rendering admission of cholinesterase inhibitors a viable treatment for cognitive and behavioral impairments as well as for hallucinations. ${ }^{[31]}$ To date, there is no pharmacological treatment 
for the cerebellar ataxia in MSA-C and therapy is limited to physiotherapy and speech therapy. ${ }^{[40]}$ One of the crucial challenges in all of these cases is the fact that by the time of diagnosis all neurodegenerative diseases have advanced significantly, not allowing early treatment. This problematic has led to various efforts in recent years regarding the early diagnosis of Synucleinopathies, however with varying success. ${ }^{[45]}$

\subsubsection{Etiology and pathogenesis}

It is still a matter of discussion, which particular influence environmental factors have on the onset of synucleinopathies. ${ }^{[45,46,47]}$ Some of the environmental factors which have been studied and suggested include exposure to pesticides, metals, organic solvents, magnetic fields, smoking, alcohol, body mass index and dietary factors. ${ }^{[48]}$ As there are several mutations in a number of genes that are involved in the development of synucleinopathies, genetic predispositions have always been included in the discussion. A common assumption is that an upregulation of $\alpha$-synuclein expression leads to pathogenesis and neuronal degeneration. ${ }^{[49]}$ Yet, since a vast majority cases are sporadic and far fewer patients have a familial history, it is often presumed, that an interplay of environmental factors and genetic susceptibility on the background of an aging brain might trigger the onset of the disease. ${ }^{[46]}$ Nevertheless, studies on mutations especially on the SNCA gene of $\alpha$-synuclein have been used to gain insight into the pathogenic mechanisms of the disease. ${ }^{[41]}$ In addition to genetic factors, epigenetic alterations, such as DNA methylation, have recently gained attention. ${ }^{[50]}$

Even after several decades of research the initiating incident of pathogenesis in neither of the diseases has been identified. The considerable overlap between symptoms hints to similarities in disease causation and pathways, but the characteristic pathology and disease progression for the various types of synucleinopathies call for a more specific description of the underlying mechanisms. ${ }^{[38,39,41]}$

The pathogenesis of PD is a complex process in which several key factors have been outlined, including mitochondrial dysfunction, oxidative and nitrative stress, microglial activation, neuroinflammation, impaired protein degradation, proteasomal impairment, overexpression and aggregation of a-synuclein, and impaired autophagy. ${ }^{[23,}{ }^{43]}$ Mechanistically, it is very difficult to distinguish between PDD or DLB respectively, however, as $A \beta$ aggregation seems to be more prominent in DLB, the mechanistic relevance of the respective protein aggregates is still a matter of discussion. ${ }^{[51]}$ Furthermore, it has been suggested, that the difference in disease progression might be linked to different spreading patterns. ${ }^{[32]}$ 
In contrast to the Lewy body diseases, studies have shown a correlation between GCI load and neuronal loss in MSA. ${ }^{[52]}$ It is still a matter of discussion, whether the oligodendrocytes, that ultimately show the inclusions, can actually express aS. As experimental results are conflicting, multiple studies have been conducted on the uptake of various aS aggregates from the extracellular environment, proving the possibility of such a pathway. Accordingly, it has also been shown, that oligodendrocytes might internalize $\alpha$-synuclein from neighboring neurons. ${ }^{[53]}$

Accordingly, a consistent characteristic of synucleinopathies, as well as for all other neurodegenerative diseases, is their multifactorial nature, incorporating the simultaneous activation of several pathogenic pathways and the concomitant failure of cellular functions. ${ }^{[32,}$

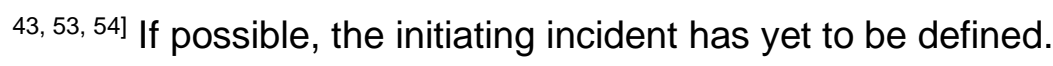

\subsubsection{The role of protein aggregation in pathogenesis}

Ever since the discovery of SNCA gene mutations in PD patients ${ }^{[42]}$ and the discovery of $\alpha S$ in $\mathrm{LB}^{[29]}$, aS-aggregation has been one of the most prominent and well-studied phenomena in pathogenesis. Over-expression of $\alpha S$ is known to drive aggregation and has thus been used in several animal models of synucleinopathies. ${ }^{[55]}$ The aggregation progresses from monomeric $\alpha S$, via transient oligomeric states, towards fibrils, that are ultimately found in the LBs, LNs or GCls. In this regard, it is specifically the aS oligomers that are believed to exert the main toxicity, leading to synaptic dysfunction and ultimately neuronal death. ${ }^{[56]}$ In addition to the toxicity of the transient protein aggregates, a prion-like spreading mechanism is proposed for synucleinopathies, where aggregated proteins act as templates, driving forth the aggregation in the infected patient. It has even been suggested, that there are specific $\alpha S$ strains, that cause the onset of MSA, DLB or PD respectively. ${ }^{[57]}$ Indeed, recent studies have shown, that such strains might exist for GCls and LBs, owing to different conformations and seeding activities the aggregates ${ }^{[58]}$ as well as different morphologies of the formed inclusions. ${ }^{[59]}$ For PD, a potential pathway of transmission has been identified in the uptake via the gastrointestinal tract and the vagus nerve towards the substantia nigra. ${ }^{[49,54,60]}$

Despite its popularity, the protein aggregation hypothesis has been challenged multiple times. One of the main arguments is the accumulation of LB pathology in AD patients ${ }^{[61]}$ and vice versa ${ }^{[62]}$ challenging the role of $\alpha S$ as a unique driver. In addition, in a study of patients showing LB pathology in their autopsy, about $23 \%$ showed neither PD, DLB or other neuronal disorders. ${ }^{[63]}$ In addition to this, brain tissue is only available post mortem, yielding information only after the initiating pathogenic events have taken place. ${ }^{[64]}$ Lastly, the recent failure of trials for aggregation modulating drugs like semagacestat (gamma-secretase inhibitor), Verubecestat (beta-secretase inhibitor) and aducanumab (monoclonal antibody 
targeting both soluble and insoluble protein) in $A D$ treatment, have raised doubts, whether protein aggregation is really a causal factor for disease. ${ }^{[65]}$

In a recent paper, Espay et al. list the aforementioned concerns and discuss three potential alternative models, that would account for non-pathogenic protein aggregates: ${ }^{[64]}$

- Protein aggregation is not pathogenic, but drives other pathogenic mechanisms

- Protein aggregates are an epiphenomenon and a neutral bystander

- Protein aggregation serves as a protective mechanism

Still, the pathogenic nature of aggregated protein is indicated by several familial mutations in the SNCA gene causing PD. ${ }^{[45]}$ Another strong argument in favor of the role of protein aggregation is the early onset of disease in case of SNCA duplication and triplication. ${ }^{[15]} \mathrm{A}$ potential protective function might explain the survival of old patients with LB pathology, assuming the protective mechanism has not been overwhelmed and rescue has been successful. ${ }^{[64]}$ In addition, this would explain the failure of drugs aiming at aggregation inhibition, as such an intervention might even have a detrimental effect. ${ }^{[64]}$

\section{3 a-synuclein}

a-Synuclein is an intrinsically disordered presynaptic 140 residue protein that is highly abundant in the human brain and enriched in the presynaptic nerve termini. ${ }^{[6]}$ The physiological concentration of was determined to be about $20 \mu \mathrm{M}$ in synaptic boutons ${ }^{[67]}$ and about $0.2 \mathrm{nM}$ in cerebrospinal fluid. ${ }^{[68]}$ Despite the great number of groups studying the protein, its exact biological function is still unknown. ${ }^{[69]}$ Because of its location and its preference for the binding to phospholipid membranes it has been linked to a regulatory role in synaptic vesicle regulation, brain lipid metabolism and neuronal survival. ${ }^{[55,70]}$ Several studies on aS knockout mice hint towards its involvement in dopamine release and control of dopamine levels. ${ }^{[71]}$ Furthermore, evidence suggests an involvement with SNARE complex formation, indicating a role in neurotransmitter release in general. ${ }^{[55]}$

\subsubsection{Monomers}

The primary structure of $\alpha$-synuclein is highly conserved in a variety of different organisms. ${ }^{[2]}$ Humans express three different isoforms: AS140, AS126 and AS112, of which the 140 residue long form is the best-known. ${ }^{[73]}$ The whole 140 residue transcript (Figure 1.3.1) is usually divided into three regions ${ }^{[74]}$ :

- The N-terminal region (residues 1-60), which is characterized by seven imperfect 11 mer repeats containing a KTKEGV consensus. The 11-mer repeat sequence is 
believed to form amphipathic helical lipid binding domains, due to its resemblance to apolipoproteins. ${ }^{[75]}$

- The hydrophobic central region (residues 61-95), often referred to as "non-amyloidcomponent" (NAC), which is strongly involved in the aggregation process.

- The C-terminal region (residues 96-140), which is highly enriched in negatively charged amino acid residues and prolines. The residues Y125, Y133, and Y136 are considered to be the signature for the $\alpha$ - and $\beta$-synuclein family.

Being a member of the intrinsically disordered protein (IDP) class, $\alpha$-synuclein is highly dynamic and flexible, lacking a defined structure. The intrinsically disordered structure is a feature often observed in proteins or protein parts involved in NDDs. ${ }^{[76]}$ NMR studies showed the lack of a secondary structure but the existence of a transient tertiary structure. ${ }^{[77]} \mathrm{A}$ very controversial topic is the existence of a native tetrameric structure, proposed by Bartels et al.. ${ }^{[78]}$. Results from various groups on this putative tetramer are conflicting ${ }^{[79]}$ and in-cell NMR recently failed to detect any evidence of a multimeric structure. ${ }^{[80]}$ Several groups however have reported, that the native helical tetramers could not be isolated from the brain tissue of mice, rats, humans or $\alpha$-syn transgenic animals. ${ }^{[81]}$ Regardless of its physiological structure, the dependence of $\alpha$-synuclein conformation on a variety of conditions has been probed. Globular forms have been found to be promoted by low $\mathrm{pH}$ and high temperature as well as various other conditions. ${ }^{\left[{ }^{82]}\right.}$ At low $\mathrm{pH}$ a-synuclein was observed to adopt a conformation in which aggregation is promoted. ${ }^{[83]}$

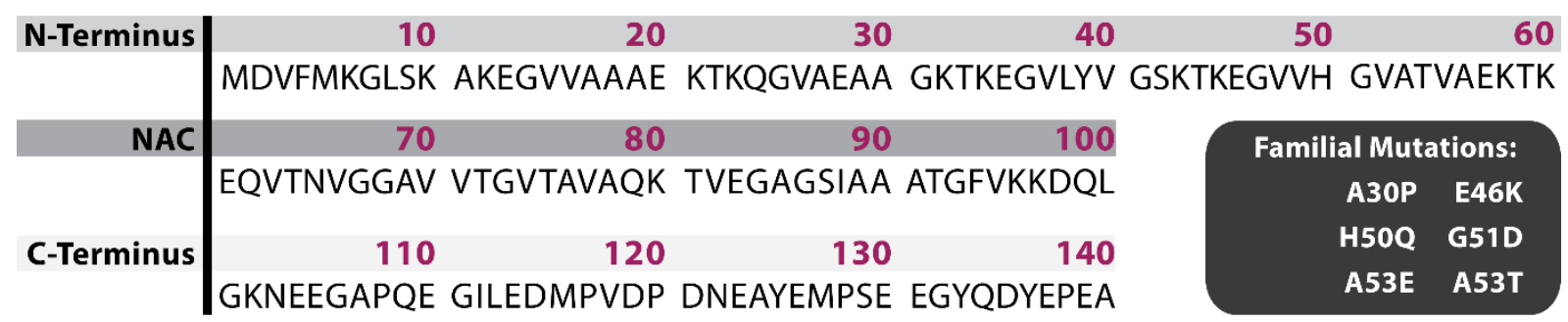

Figure 1.3.1: Primary Sequence of aS with its three characteristic domains highlighted. Bottom right: Familial Mutations related to early onset of disease.

\subsubsection{Oligomers}

Current theories on the involvement of $\alpha \mathrm{S}$ aggregation in disease progression, assume that protein oligomers exert the major toxicity. ${ }^{[84]}$ These oligomers consist of two and more monomers and form diverse structures that highly depend on the conditions under which they are formed. ${ }^{[85]}$ Besides the putative $\alpha$-helical tetramer outlined earlier, most oligomers 
are rich in $\beta$-sheet content and display a high degree of heterogeneity. ${ }^{[86]}$ Although oligomers assemble spontaneously during fibril formation, their formation can be induced by various agents, such as fatty acids, metals and various compounds, including Dopamine. ${ }^{[87]}$

Due to their transient nature and low population levels during all stages of the aggregation process, structural studies on aS oligomers are limited. Using Size Exclusion Chromatography, Lorenzen et al. succeeded in the isolation of two distinct oligomeric species, consisting of 18 and 29 monomers respectively, that inhibit fibril formation. ${ }^{[88]}$ Despite their size difference, these oligomers share the same content in antiparallel $\beta$-sheet content and were later shown to adopt a cylinder-like shape with a hollow core. ${ }^{[89]}$ The calcein release and neuronal cell toxicity observed for these aggregates was attributed to insertion of their structured region into phospholipid bilayers, as detected by use of paramagnetic labelled phospholipids (Figure 1.3.2 A). ${ }^{[00]}$ Such an insertion had been confirmed earlier by Comellas et al. on aS oligomers prepared in the presence of phospholipid bilayers through ${ }^{1} \mathrm{H}$ spin diffusion from lipids to protein in solid-state NMR (ssNMR). ${ }^{[11]}$ The core region of oligomers is resistant to changes in temperature and $\mathrm{pH}$ and only disintegrates slowly at high concentrations of urea. ${ }^{\left[{ }^{92]}\right.}$ At low temperatures fibrils of $\alpha S$ release on-pathway oligomers, that induce pore formation in planar lipid bilayers (Figure 1.3.2 B), displaying the dynamic nature of the aggregation process. ${ }^{\text {[93] }}$

A

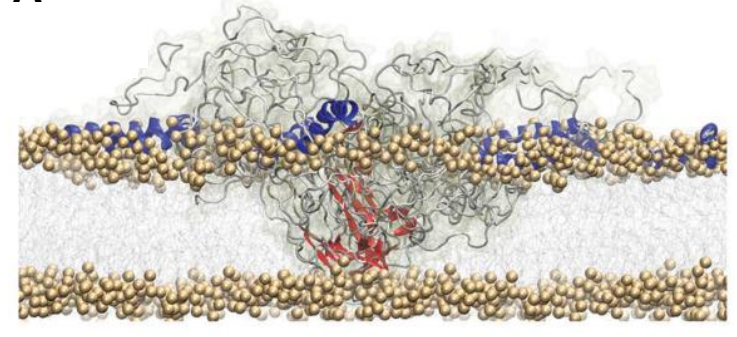

\section{B}

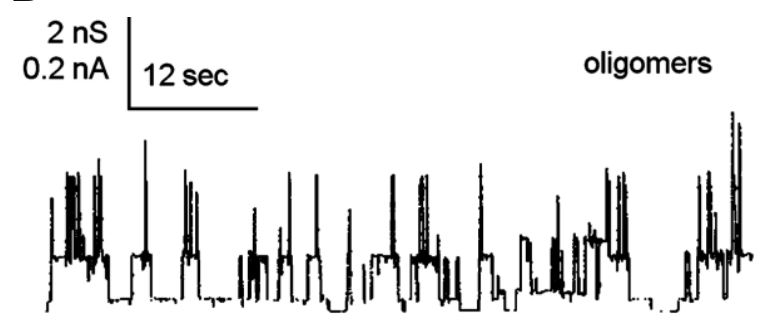

Figure 1.3.2: A: Insertion of an aS oligomer into a phospholipid bilayer as suggested by Fusco et al. ${ }^{[90]}$; B) Pore forming activity displayed by aS oligomers demonstrated by black lipid membrane (BLM) measurements. ${ }^{[93]}$

Pore formation is believed to play a major role in the toxicity of aS oligomers, alongside a thinning of cell membranes and a potential detergent-like mechanism, all leading to membrane disruption. ${ }^{94,}{ }^{95]}$ Other possible cytotoxic mechanisms encompass the generation of reactive oxygen species, mitochondrial dysfunction and dysregulation of calcium homeostasis. ${ }^{[86]}$ 


\subsubsection{Fibrils}

Eventually, aS oligomers restructure, elongate and form amyloid fibrils, as depicted in Figure 1.1.2. This process likely occurs through internal rearrangement and subsequent recruitment of monomers, although the exact mechanism is unclear as heterogeneity of the possible pathways makes analysis difficult. ${ }^{[15]}$ In the amyloid state, the protein obtains a highly ordered, tightly packed, rigid cross $\beta$-structure. ${ }^{[96]}$ Depending on the aggregation conditions and due to the multiple pathways, $\alpha \mathrm{S}$ fibrils show significant polymorphism. ${ }^{[15]}$

In recent years, significant progress has been made regarding the determination of fibril architecture, leading to the publication of structures solved by ssNMR ${ }^{[97]}$, and cryo-electron microscopy (cryo-EM). ${ }^{[98,99,100,101]}$ As depicted in Figure 1.3.3 the structured region is uniformly limited to the NAC region and parts of the $\mathrm{N}$-terminus, while the $\mathrm{C}$-terminus remains unstructured. Despite several reported differences in monomer arrangement within the filament sections, three key motifs were identified in the depicted structures. The first motif to be identified by ssNMR (PDB Entry 2N0A, not shown) was dubbed the "Greek-key", referring to the characteristic fold of the monomers. ${ }^{[97]}$ This fold was later confirmed by cryoEM (PDB 6H6B, 6A6B), albeit with a dimeric structure instead of the previously reported single strand. ${ }^{[98,101]}$ Expanding on this, $\mathrm{Li}$ et al. described two distinct polymorphs, one of which carrying a Greek-key arrangement with a longer pitch of about $9.2 \AA$ (PDB 6CU7) and the other with a different monomer arrangement and a shorter pitch of about $4.6 \AA$ (PDB 6CU8). ${ }^{[99]}$
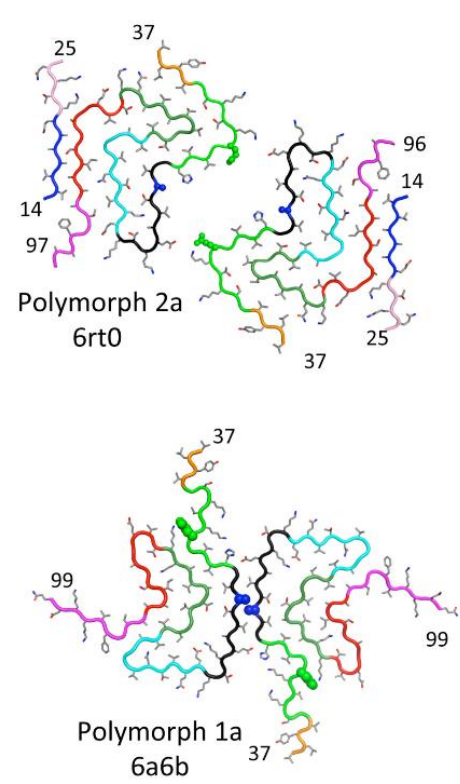
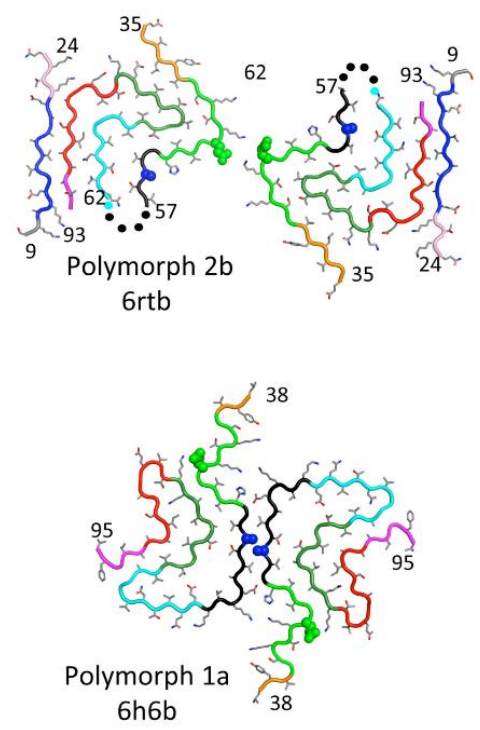
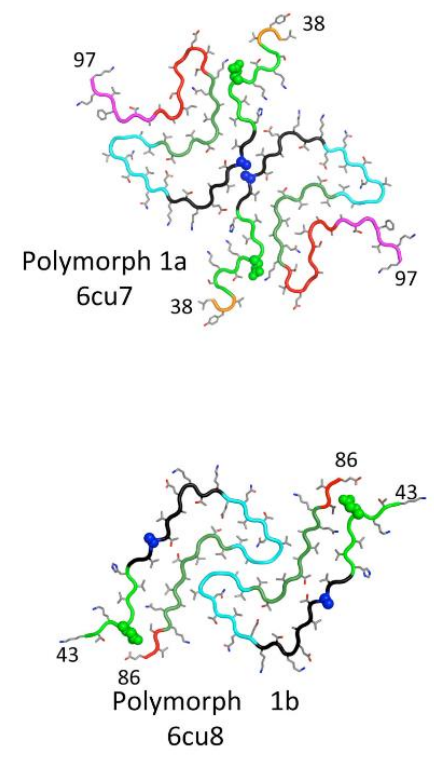

Figure 1.3.3 Published structures of aS polymorphs; adopted from Guerrero-Ferreira et al. 2019. ${ }^{[100]}$ 
Recently, another set of polymorphs was characterized, showing significant contrast in filament attachment. ${ }^{[100]}$ The newly proposed polymorphs bear connective salt bridges, whereas the Greek key and twister polymorphs form a broad interface of interlocking hydrophobic amino acid sidechains. Noticeably, in all structures charged amino acid residues face outwards and hydrophobic residues tend to face the inside of the filament core.

It remains elusive, however, whether the described polymorphism has a direct correlation to disease progression. In the case of Pick's and Alzheimer's Disease two distinct folds were observed for tau-fibrils from brain derived patient material, although it has to be considered that tau differs in the number of repeats depending on the disease. ${ }^{[102,103]}$ As mentioned earlier, such disease specific characteristics have been indicated in studies of MSA and PD material as well. ${ }^{[58,59]}$ So far, structures of aS fibrils derived from patient material have not been reported. Fibrils have been shown to seed and certain animal studies that support a prion like behavior, however mechanisms like aggregate propagation and cell uptake need to be elucidated, before strains such as in Prion Diseases (PrDs) can be accepted as a given. ${ }^{[12,57]}$ A contribution by Karpinar et al. showed in a series of animal models that fibrils are not necessary for $\alpha$-Synuclein toxicity corroborating the toxicity of oligomers. ${ }^{[104]}$

Several factors drive fibril formation. The tight packing of monomers results in the release of water molecules, resulting in an entropy gain. This entropy gain is combined with the attractive van der Waals energy between the interlocked residues and a mutual polarization of the hydrogen bonds between the filament sheets. ${ }^{[105]}$ As can be seen in Figure 1.3.4 side chains along the fibril axis can form ladders, further adding to the stability of the filaments.
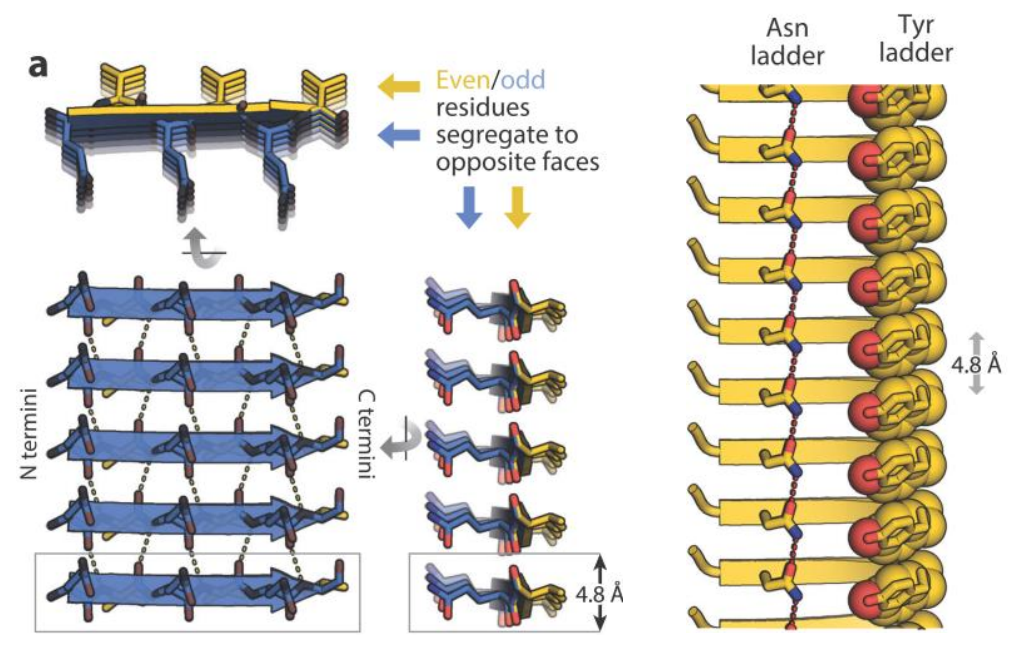

Figure 1.3.4: Stabilizing interactions between sheets in a cross $\beta$-structure. Left: hydrogen bonding between fibrils sheets resulting in the alignment of residues to ladders. Right: Hydrogen bonding and $\pi-\pi$-stacking along ladders, adding to fibril stability. ${ }^{[105]}$ 


\subsection{Amyloid Aggregation Kinetics}

Like for any other amyloidogenic protein, aS aggregation follows the principles of nucleated self-assembly, as outlined in Chapter 1.1. ${ }^{[106]}$ The simplest model for nucleated polymerization assumes direct formation of fibril-like aggregates from monomers, which then elongates. Alternatively, monomers form less structured aggregates, such as oligomers, which eventually restructure to form nuclei in a process called nucleated conformational conversion. ${ }^{[15]}$ Both of these processes outlined above proceed through energetically unfavorable transition states, resulting in a slow initial formation of aggregates, reflected in the so called "lag phase" (Figure 1.4.1). ${ }^{[17]}$ Once a certain nucleus concentration is achieved, elongation of these nuclei into fibrils progresses rapidly until slowing down after an equilibrium is reached. This order of events is reflected in the characteristic sigmoidal growth kinetics. ${ }^{[107]}$ In the presence of preformed fibrils, so called seeds, the lag phase is reduced and with growing seed concentration eventually starts with the growth phase. ${ }^{[108]}$
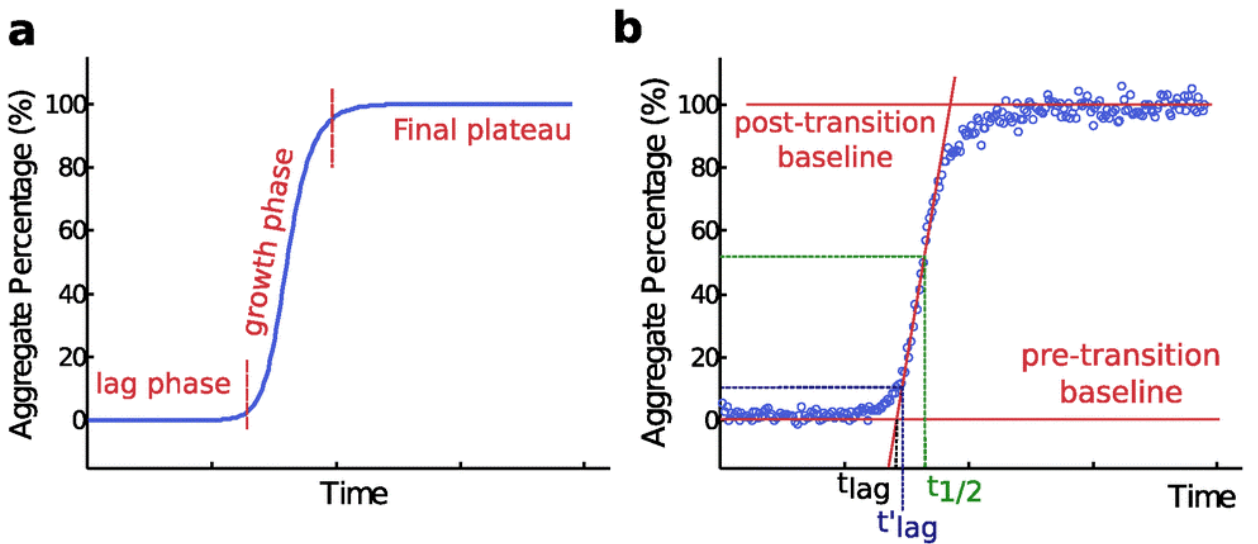

Figure 1.4.1: Characteristic aggregation curve for amyloid fibril formation: a: phases of aggregation b: definition of $\mathrm{t}_{\text {lag, }}$, tag' $\left(10 \%\right.$ of final aggregate) and $t_{1 / 2}(50 \% \text { of final aggregation })^{[17]}$

The parameters of the kinetic process are strongly influenced by the conditions under which the aggregation takes place. Relevant parameters include protein concentration, buffer conditions, $\mathrm{pH}$ values, temperature, agitation speed and type and presence of solution-air interfaces. ${ }^{[106]}$ Recently, the influence of phospholipids on $\alpha S$ aggregation kinetics has gained growing attention, identifying lipid to protein (L/P) ratio, head group charge and acyl chain length as important influences. ${ }^{[109,110]}$

\subsubsection{Interaction of $\alpha$-synuclein with phospholipid membranes}

Due to its high affinity for negatively charged phospholipids, aS is found in a free and membrane bound state in synaptic termini. ${ }^{[111]}$ Upon membrane binding the first $100 \mathrm{~N}$ terminal residues of the protein adopt an amphipathic helix conformation, while the 
C-terminus remains unstructured. ${ }^{[112]}$ Deletion of the first $20 \mathrm{~N}$-terminal residues highlighted their necessity for membrane binding. ${ }^{[113]}$ Mutation of lysines $\mathrm{K} 10$ and $\mathrm{K} 12$ as well as K21 and K23 to glutamic acid confirmed these results and further showed the importance of the positively charged lysine residues for membrane binding. ${ }^{[114]}$ In a combined approach of ssNMR and solution NMR, Fusco et al. showed, that residues 1-25 have an anchoring function, while the rest of the membrane binding domain exhibits a more dynamic interaction (Figure 1.4.2). ${ }^{[115]}$ The exact structure of the membrane bound helix is not clear. Whereas some studies report two anti-parallel $\alpha$-helices with a short kink $^{[116]}$, others favored an extended helix. ${ }^{[117]} A$ recent NMR spectroscopic study on $\alpha S$ in the presence of nanodiscs provided arguments for both conformations. ${ }^{[118]}$

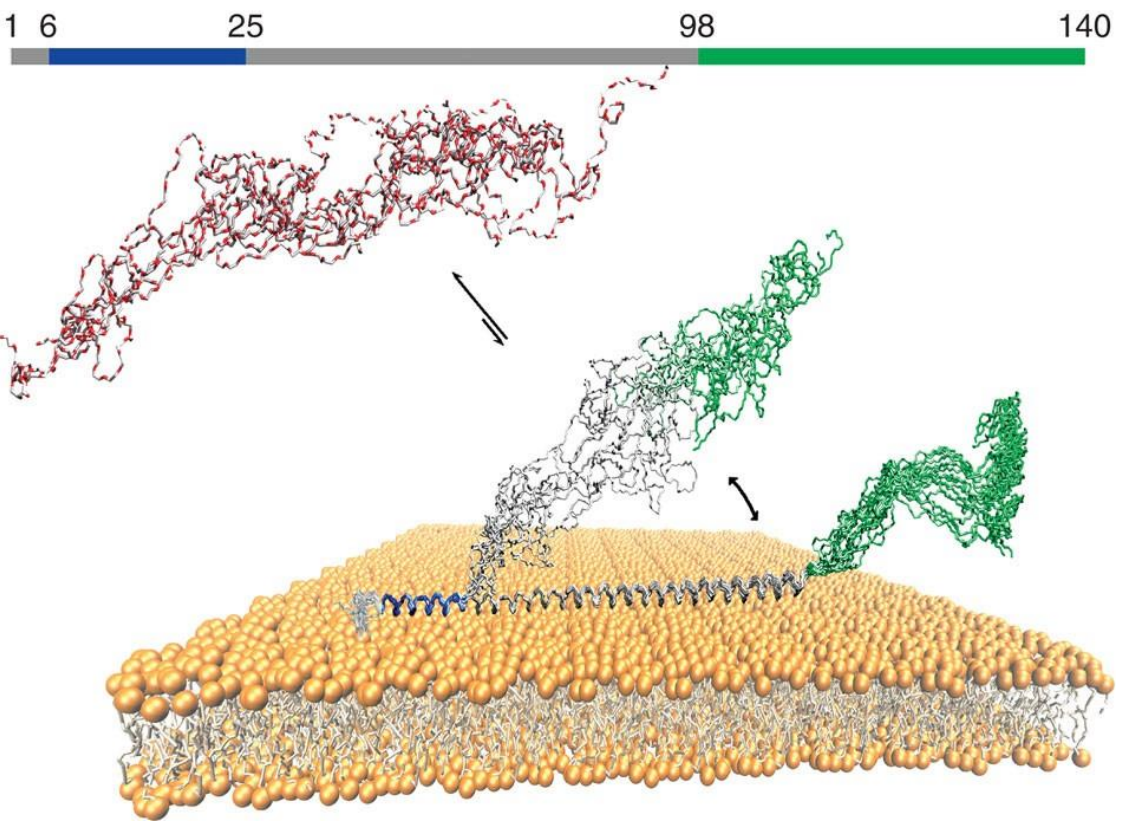

Figure 1.4.2: Schematic illustration of the different roles of the three regions of $\alpha$-synuclein regarding its binding to lipid membranes. The first 25 $\mathrm{N}$-terminal residues exhibit a tight anchor function, while the NAC sequence binds dynamically. The Cterminus remains unbound and unstructured. ${ }^{[15]}$

\subsubsection{Protein Misfolding Cyclic Amplification (PMCA)}

Protein misfolding cyclic amplification was initially reported by Soto and coworkers as a useful way to rapidly convert cellular Prion Protein $\operatorname{PrP}^{\mathrm{C}}$ into the disease relevant scrapie form PrPSc by addition of small amounts of brain derived PrPSc. ${ }^{[119]}$ Although this method was developed for Prion Protein, it has since been used for the amplification of seed material from various other NDD related proteins, such as $A \beta 1-42^{[120]}$, Tau ${ }^{[121]}$ and $\alpha$-synuclein ${ }^{[122]}$.

The procedure is generally the same and involves incubation of a template, the protein aggregate, together with a substrate, the protein monomer, which are then subjected to repeated cycles of short sonication, each followed by a long quiescent phase. ${ }^{[123]}$ During the resting periods the templates are elongated by addition of monomeric protein at the nucleation sites. In the subsequent sonication phase the aggregates are broken down, to create multiple small template fragments. These cycles of fragmentation of elongation finally 
yield high amounts of the amplified seed material. ${ }^{[124]}$ One of the rate limiting factors in protein aggregation is the availability of nucleation sites ${ }^{[125]}$, which is circumvent by the generation of a multitude of sites through sonication, resulting in a significantly increased aggregation rate. The resulting advantages of the technique include significantly shortened reaction times, lower sample demand and high reproducibility. ${ }^{[122]}$

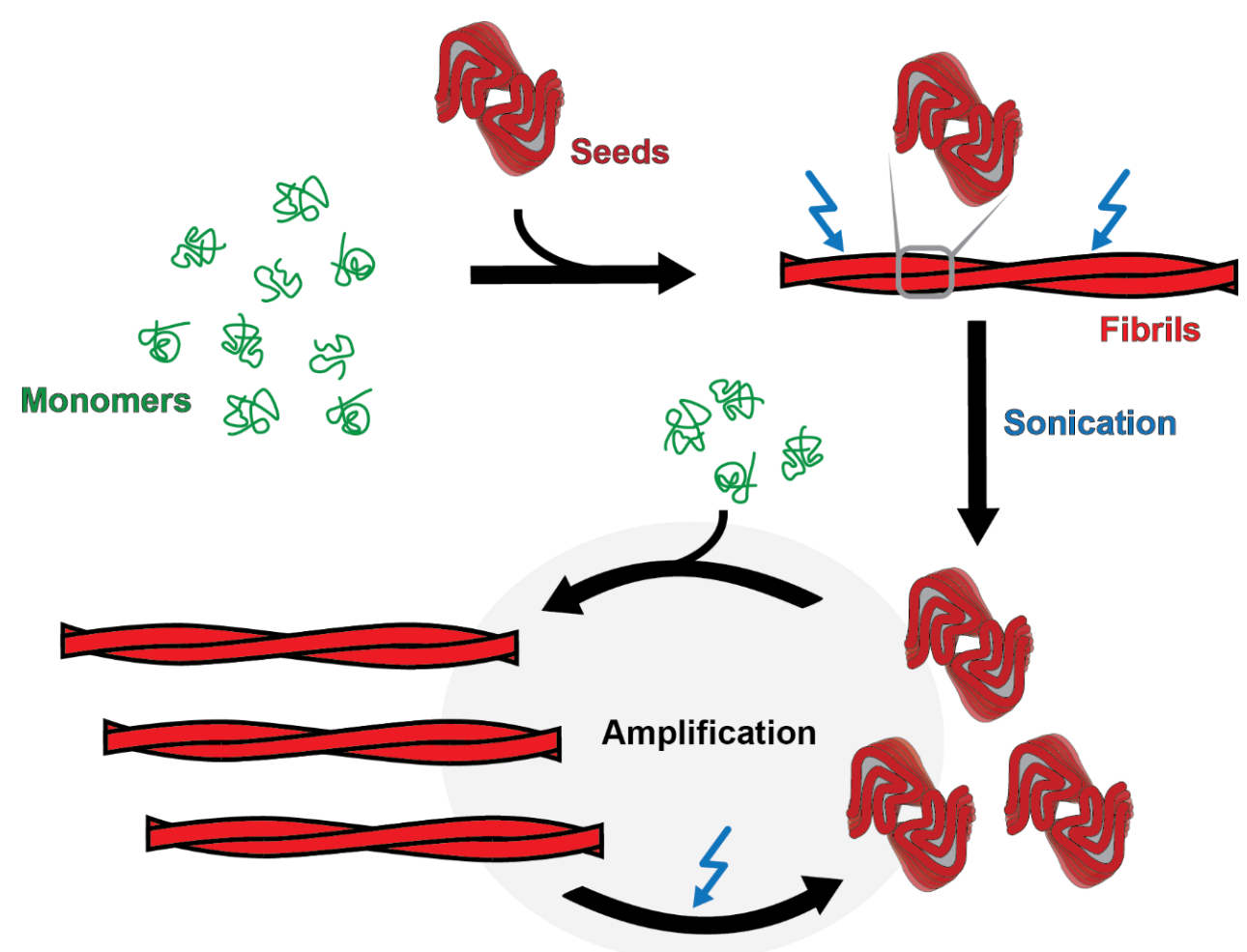

Figure 1.4.3: Schematic representation of the processes taking place during PMCA. The incubated seeds act as a template for aggregate growth. Upon sonication new smaller fragments are generated, allowing faster aggregation due to an increase in nucleation sites. Repetition of this cycle allows rapid amplification of the initial seed. Adopted from [119].

This phenomenon still holds true in the absence of seeding material (Figure 1.4.4). ${ }^{[122]}$ The resting periods however seem to be as crucial as the sonication periods, as it was found, that an alternation of agitation and quiescence results in a shorter lag phase, than uninterrupted

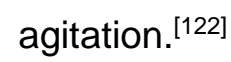

Several studies have been conducted, aiming at the clinical application of PMCA for rapid diagnosis of protein aggregates, using various sources of patient material, such as gastrointestinal biopsies ${ }^{[126]}$ and cerebrospinal fluid. ${ }^{[127]}$ The successful application of PMCA has led to the development of modified versions ${ }^{[128]}$ and new techniques such as real-time quaking-induced conversion (RT-QUIC). ${ }^{[129]}$ Recently, the biotechnology company Amprion confirmed acceptance of their PMCA technology in the FDA Breakthrough Devices Program, aiming at the market rollout of new technologies. ${ }^{[130]}$ 


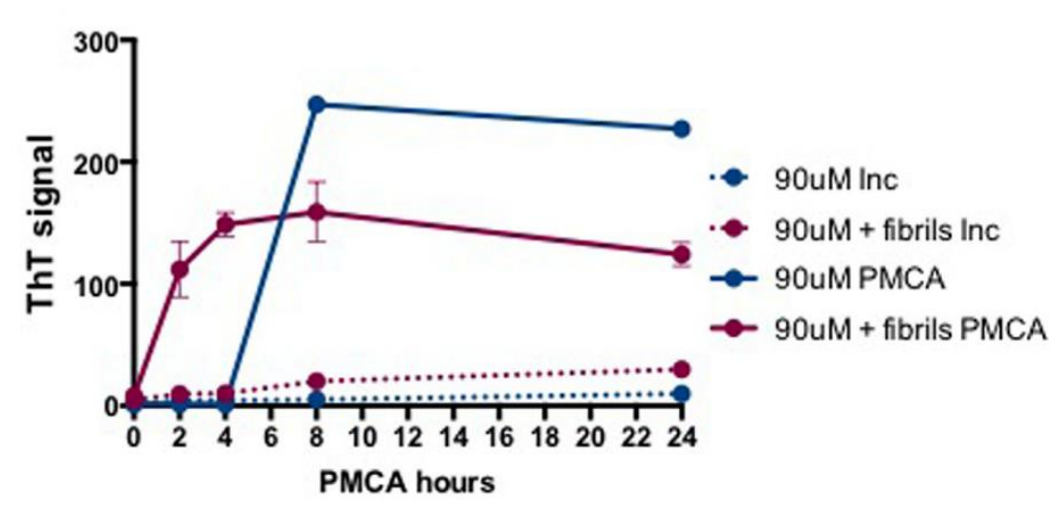

Figure 1.4.4: Aggregation kinetics of reactions carried under PMCA-conditions and regular incubation/shaking both in the presence and absence of seeds. Aggregation proceeds far more quickly under PMCA conditions even when no seeds are present. ${ }^{[122]}$

\subsection{Anle138b as a potential disease modifying agent}

The lack of disease modifying treatments in NDDs calls for the development of drugs, which are able to modify the toxic processes in these diseases. In a screening of 20.000 drug-like compounds using a molecular SIFT assay and in an anti-prion cell culture assay, 3,5diphenyl-pyrazole (DPP) was found as a lead structure for the inhibition of prion protein aggregation. ${ }^{[131]}$ Among a library of about 150 DPP-derivatives, anle138b (Figure 1.5.1) displayed the highest efficacy in inhibition of protein aggregation in both prion disease and PD mouse models.<smiles>Brc1cccc(-c2cc(-c3ccc4c(c3)OCO4)[nH]n2)c1</smiles>

Figure 1.5.1: Chemical structure of anle138b

Further studies on mouse models of $\mathrm{MSA}^{[132,133]}, \mathrm{PD}^{[134]}, \mathrm{AD}^{[135]}$ and tauopathies ${ }^{[136]}$ underlined the potential of anle138b as an aggregation modulator in NDDs. Using the autofluorescence of the compound it was shown that anle138b binds to aS fibrils with high affinity (Figure 1.5.2). ${ }^{[137,138]}$ By means of atomistic MD simulations Matthes et al. showed that anle138b binds with high affinity to the backbone of $\beta$-sheet rich oligomers of decapeptide segments from aggregation prone regions of $\mathrm{hTau} 40, \mathrm{~A} \beta$, hIAPP , and Sup35N (Figure 1.5.2). ${ }^{[139]}$ 

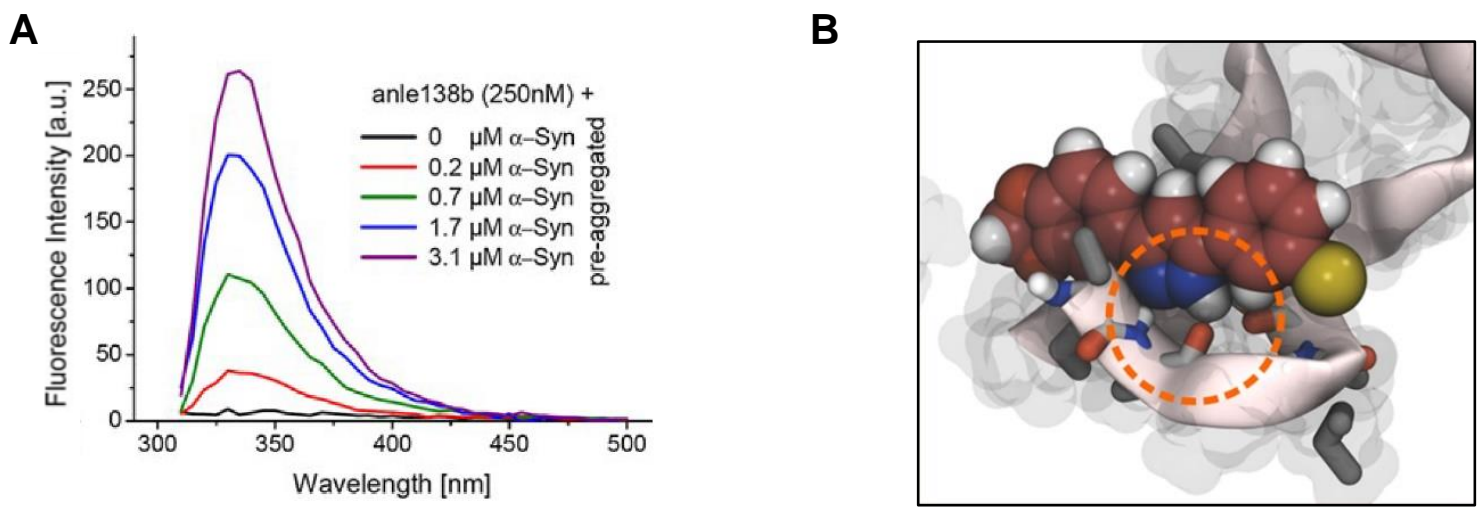

Figure 1.5.2: Interaction of anle138b with protein aggregates revealed from biophysical studies; A: auto-fluorescence of anle138b when binding to aS-fibrils ${ }^{[137]}$; B: Binding of anle138b to the backbone of $\mathrm{hTau} 305-314$ oligomers as found in MD-simulations. ${ }^{[139]}$

One of the key mechanisms postulated for anle138b is the modulation of toxic oligomers, especially pore forming oligomers. ${ }^{[131]}$ When membranes of 1,2-Diphytanoyl-sn-Glycero-3Phosphatidylcholine were doped with anle138b, pore formation by aS oligomers was inhibited, leading to an abrupt stopping of conductance in black lipid membrane (BLM) measurements (Figure 1.5.3). A similar effect was reported for $A \beta_{1-42}$ oligomers. ${ }^{[135]}$
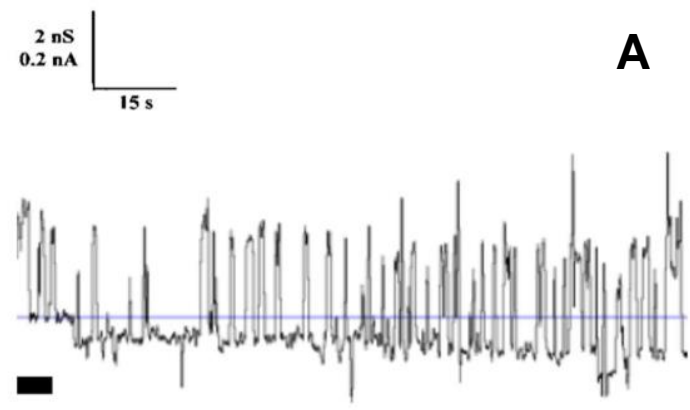

A

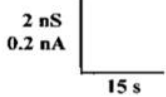

$1 \mathrm{~min}$

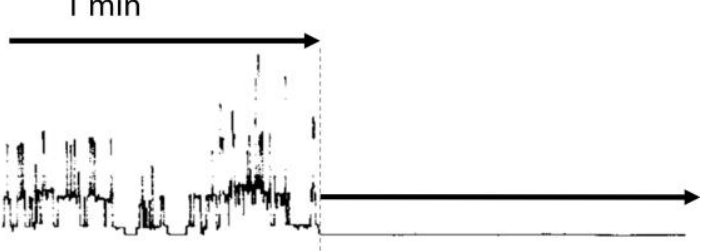

Figure 1.5.3: A): Pore formation of aS oligomers on 1,2-Diphytanoyl-sn-Glycero-3-Phosphatidylcholine bilayers as measured by BLM shows vigorous pore formation; B) Complete inhibition of aS pore formation in the presence of anle138b, approx. 1 min. after addition of protein (measured by Roland Benz, Jacobs University Bremen, unpublished results).

\subsection{Anle138b in Phospholipid Membranes}

Due to its high hydrophobicity anle138b is almost insoluble in water, resulting in concentrations in the nanomolar range. ${ }^{[137]}$ To achieve high compound to protein ratios, the use of phospholipids as carriers of anle138b is imperative. In the course of my master thesis in the lab of Prof. Griesinger at the MPIBPC, the behavior of the compound in vesicles was studied by means of NMR Spectroscopy. NOESY spectra using very short mixing times of $\mathbf{T}_{\mathbf{m}}=3 \mathrm{~ms}$ reveal a preferred conformation of anle138b inside the bilayer. When investigating the cross correlation of proton $\mathrm{H}_{4}$ of the central pyrazole ring to its neighboring nuclei, it can 
be seen that the cross peak intensity resulting from coupling to $\mathrm{H}_{5}$ is much greater than the intensity of the cross peak to $\mathrm{H}_{6}$ and similarly the intensity of the cross peak to $\mathrm{H}_{2}$ is much greater than that to $\mathrm{H}_{1}$. This difference must arise from closer proximity of the nuclei, brought about by conformational rigidity of the molecule. Rotation about the bond vectors seems to be restricted in the lipid environment, so that energy barriers are increased.

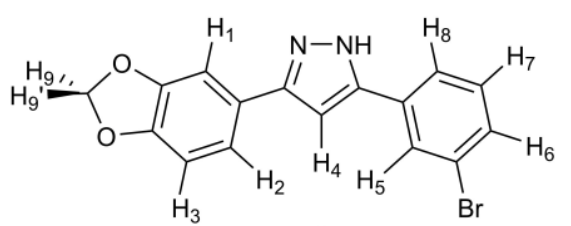

A

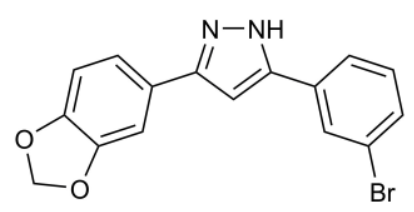

C<smiles>Brc1cccc(-c2cc(-c3ccc4c(c3)OCO4)n[nH]2)c1</smiles>

B

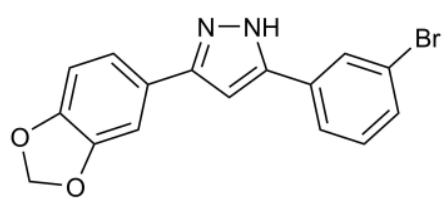

D

Figure 1.6.1: The four possible rotamers of anle138b assuming coplanarity of the aromatic rings; NOESY measurements reveal a predominant population of conformation A. Numeration of protons chosen in this work depicted for this conformer.

Figure 1.6.1 depicts the four possible rotamers of anle138b, assuming coplanarity of the aromatic rings with the central pyrazole. The assumption of planarity is favored by alignment of the $\pi$-systems, but should only be seen as a rough approximation, as the rings can also tilt out-of-plane. ${ }^{[140]}$ From the NOESY cross peak intensities ratios of $\mathrm{I}_{4-2} / \mathrm{l}_{4-1}=4.6 \pm 0.8$ and of $\mathrm{I}_{4-5} / \mathrm{I}_{4-6}=4.0 \pm 0.8$ were determined. These ratios are interpreted as the populations of the respective rotamers of the two aromatic rings. This leads to $A$ as the main conformer inside the lipid bilayer, with minor populations of conformers $B$ and $C$ and presumably very low populations of $D$ as the apparently least favorable conformation.

To assess the localization and orientation of anle138b inside the bilayer of the SUVs, the relative NOE transfer rate between phospholipid and compound resonances were approximated at $300 \mathrm{~ms}$ on the assumption of an isolated spin pair model. ${ }^{[141]}$ Since spin diffusion is highly active in the spins of the bilayer system, the profiles of the rates for the individual protons of the compound are very similar. However, globally the highest relaxation rates are found for correlation with $\alpha$ - and $\beta-\mathrm{CH}_{2}$ groups of the acyl chains of the lipids. Slightly lower rates are observed for the protons adjacent to the olefinic carbon atoms and the neighboring $\mathrm{CH}_{2}$ groups as well as the glycerol backbone. Very low rates are observed for cross relaxation rates from the terminal $\omega-\mathrm{CH}_{3}$ group and the various $\mathrm{CH}_{2}$ groups of the carbon chain along with thee protons of the choline head group of POPC. 
This leads to the conclusion, that anle138b is located closely below the lipid-water-interface, rather than deeply incorporated in the membrane center. The compound appears to favor interaction with the polar carbonyl groups of the fatty acid residues, in agreement with the observed solubility of the compound in polar solvents and the complete insolubility in apolar solvents. A single preferred orientation of the compound relative to the bilayer normal is difficult to pinpoint, given the homogeneity of the cross-relaxation profiles. However, based on its preferred conformation, it seems likely that the compound forms hydrogen bonds to the water molecules, while burying the large hydrophobic bromine atom inside the bilayer.
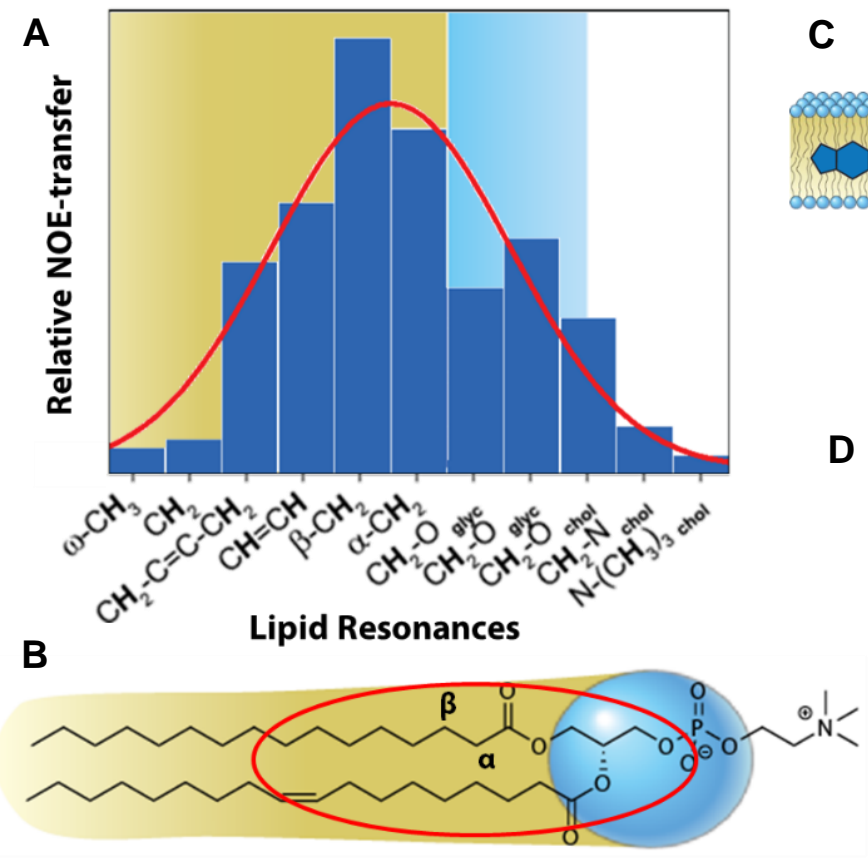

Figure 1.6.2: A) Relative NOE-transfer rates from lipid proteins to anle138b measured by NOESY at $\mathrm{Tm}=300 \mathrm{~ms}$; the background was color coded by the location of the interaction site in the lipid structure (B). C): Preferred conformation of anle138b in phospholipid bilayers as revealed by NOESY at $\mathrm{T}_{\mathrm{m}}=3 \mathrm{~ms}(\mathrm{D})$. 


\section{Aims and Scope}

The success of anle138b in vivo calls for an elucidation of the underlying mechanism in vitro. Biophysical studies hint towards a modulation of oligomeric protein aggregates in the disease process. An in-depth understanding of the molecular interactions of anel138b with these species will not only help in the improvement of lead structures, but also further our understanding of the formation and the disease relevant properties of protein aggregates. Hence, the aim of this thesis is the identification and characterization of interactions between anle138b and $\alpha S$ oligomers and fibrils.

For this purpose, a protocol based on PMCA methodology was developed, allowing the enrichment of $\alpha S$ oligomers in the presence of phospholipids. The aggregation of $\alpha S$ under these conditions was studied thoroughly using a variety of methods, including solution NMR, Fluorescence and Circular Dichroism (CD) -spectroscopy as well as Atomic Force Microscopy (AFM) and Transmission Electron Microscopy (EM).

In collaboration with Loren Andreas and Riza Dervişoğlu solid-state NMR was employed for the structural characterization of aS intermediates as well as fibrils. Sequence specific assignments were performed on the basis of proton detected 3D spectroscopic experiments. The fibril structure was discussed on the basis of published data. Assignments on aS allowed structural conclusions about the on-pathway intermediates formed by PMCA.

Lastly the interaction of the previously mentioned aggregates with anle138b was investigated by solid-state NMR. DNP-enhanced ssNMR at $100 \mathrm{~K}$ was used to identify binding sites in fibrils and oligomers. In collaboration with Kris Runge, photoactive derivatives of anle138b were used in photoaffinity studies, complementing the results from ssNMR. Based on these experimental findings, MD-simulations were carried out by Dirk Matthes, allowing atomistic insights into the binding mode of the compound. 


\section{Results}

\subsection{Oligomer formation in the presence of phospholipids by PMCA}

\subsubsection{Kinetics of aS Aggregation under PMCA Conditions}

In order to be able to enrich oligomeric species and to fully understand the aggregation process, the kinetics of $\alpha$-synuclein aggregation were investigated under several conditions. Owing to the high hydrophobicity of anle138b, phospholipids are necessary to achieve high compound to protein ratios in aggregation assays. Using vesicles of DPPA and DPPC, Zhu et al. showed that phospholipids enhance aggregation of $\alpha S$ at low L/P ratios and slow down until eventually inhibiting the process at high L/P. ${ }^{[142]}$ However, since this influence is dependent on numerous factors, such as head group charge, acyl chain length and buffer composition, the kinetics have to be investigated for each system individually. ${ }^{[110]}$

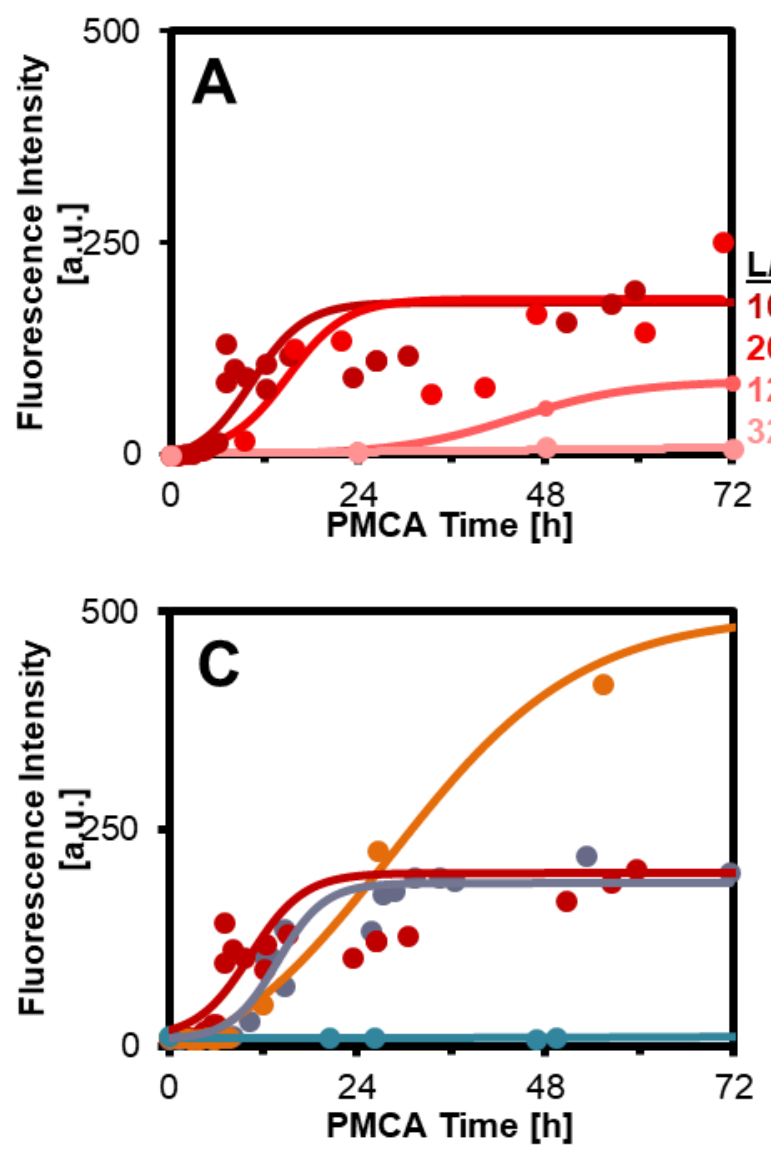

$\mathbf{B}$\begin{tabular}{|c|cc|}
\hline L/P & $\mathbf{t}_{\text {lag }}$ [h] & Th $_{\max }$ [a.u.] \\
\hline 0 & 8.8 & 218 \\
10 & 4.4 & 212 \\
20 & 9.4 & 184 \\
120 & 28.1 & 88 \\
320 & $\sim 96$ & 37 \\
\hline
\end{tabular}

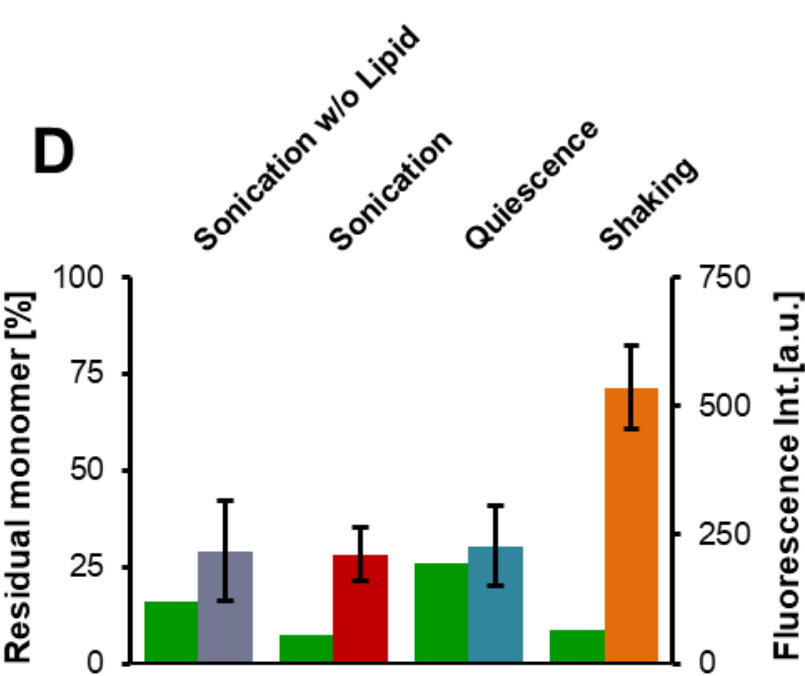

Figure 3.1.1: A) Aggregation of $\alpha S$ in the presence of increasing amounts of phospholipids, ; B) Lag times and maximum ThT values obtained from fitting sigmoidal function to kinetic data; C) Influence of agitation of the aggregation kinetics of aS aggregation: sonication without lipids (purple), sonication (red), quiescence (teal) and shaking (orange) in the presence of phospholipids at $\mathrm{L} / \mathrm{P}=10 ; \mathrm{D}$ ) Comparison of residual monomer (green) with the maximum observed ThT values for aggregation data in $\mathrm{C}$. 
The aggregation of $\alpha$-synuclein was investigated in the presence of SUVs consisting of POPA and POPC (1:1) at various L/P ratios under PMCA conditions at $37^{\circ} \mathrm{C}$ (Figure 3.1.1 A). The lag time of about $9 \mathrm{~h}$ in the absence of lipids was significantly shortened in the presence of $L / P$ ratio lower than 20 , while high $L / P$ increased the lag time to several days (Figure 3.1.1 B). At a L/P ratio of 320 it takes more than a week to show even minor levels fluorescence intensity, however complete inhibition as reported in several other publication was never achieved. ${ }^{[109,142]}$

Since, so far, there are no studies on a-synuclein aggregation in the presence of pure phospholipids under PMCA conditions, aggregation kinetics were compared to other agitation methods such as orbital shaking and quiescence. (Figure 3.1.1 B). When comparing orbital shaking at $300 \mathrm{rpm}$ to PMCA conditions at a L/P ratio of 10 , it is evident, that the lag time is significantly shorter under sonication while the maximum fluorescence intensity is decreased. Under quiescent conditions it takes approximately one week to show measurable amounts of ThT fluorescence and almost two weeks to reach plateau values similar to those obtained under sonicating conditions. Interestingly, the value of the maximum ThT fluorescence does not correlate with the monomeric protein found by NMR spectroscopy at the end of the aggregation. As can be seen in Figure 3.1.1 C for shaking as well as PMCA conditions in the presence of lipids, the final ThT fluorescence enhancement varies considerably and cannot be explained with the amount of fibrilization estimated by quantification of the residual monomer content. For PMCA conditions, residual monomeric protein is found to be approximately $7 \%$ of the initial concentration, whereas the ThT values in the case of shaking are more than twice as high, despite $9 \%$ of residual monomer. For quiescent conditions, these findings are reversed, with ThT values being comparable to those for PMCA conditions, however residual monomer is approximately $25 \%$. As ThT fluorescence does not seem to be quantitative, values are subsequently taken as an indicator for relative, rather than absolute concentrations. 


\subsubsection{Quantification of monomeric aS binding to phospholipids}

In order to fully describe the kinetics of aS aggregation in the presence of lipids under PMCA conditions, ThT fluorescence assays where complemented by solution-state NMR. 2D correlation spectra recorded by solution-state NMR were used to quantify monomeric protein, similar to studies previously carried out by $1 \mathrm{D}^{1} \mathrm{H}$ NMR. ${ }^{[143]}$

In the presence of phospholipids special care must be taken when interpreting spectra, since binding of aS to the lipid bilayers changes their appearance. Figure 3.1.3 A shows the intensities per residue along the aS sequence as derived from the backbone ${ }^{1} \mathrm{H}-{ }^{15} \mathrm{~N}$ crosspeaks in a ${ }^{1} \mathrm{H}^{-15} \mathrm{~N} \mathrm{HSQC}$ spectrum of uniformly ${ }^{15} \mathrm{~N}$-labelled aS in the presence of increasing amounts of SUVs of POPA and POPC (1:1). Upon addition of lipids, the protein signals along the N-terminal and NAC-region of aS are attenuated, while the C-terminal domain remains relatively unchanged. Only at high L/P ratios the $\mathrm{C}$-terminus shows minor attenuation, while signals form the $\mathrm{N}$-terminal domain start to drop below the detection limit. The reason for this behavior is formation of a slowly tumbling $\alpha$-helical structure by residues 1-100 of the monomeric protein on the negatively charged membrane. Due to its negative charge the Cterminal domain remains unbound and dynamic and is therefore still detected. The fact that there are neither chemical shift changes nor line broadening indicates a slow exchange between the bound and the unbound states for those first 100 residues of the aS sequence. This behavior has previously been described by Bodner et al... ${ }^{[144]}$ At L/P $=10$ the intensities of residues 101-140 are very close to the free monomer in solution and monomer bound to phospholipid. Therefore, these residues were used for derivation of the monomer concentration, as intensities from residues 1-100 originate from the unbound species only and subsequently a large portion of the protein in solution would be missed. Therefore, hereafter, the monomer concentration is taken to be proportional to the relative intensities of the C-terminal domain.

Upon aggregation, signal intensities in the ${ }^{1} \mathrm{H}-{ }^{15} \mathrm{~N}$ HSQC spectra decrease, as large aggregates tumble too slowly to be detected by solution-state NMR (Figure 3.1.2 B). In the beginning $(0 \mathrm{~h})$ the intensities show an attenuation profile as outlined earlier, however once initial aggregation occurs (4 h), the intensities of residues $101-140$ decrease to about $90 \%$ of their initial values, while the rest of the spectrum seems unchanged. Once aggregation progresses further $(6 \mathrm{~h})$, the profile characteristic to lipid binding is lost completely and and the signal intensities of the $\mathrm{C}$-terminal domain drop to the level of the intensities observed for the NAC-region. This development progresses and finally reaches a steady state after about $13 \mathrm{~h}$, with a final residual monomer concentration of about $7 \%$. Judging from this 
observation, it appears that the bound aS species is consumed into a larger aggregate, with the conversion of newly bound protein being too fast to be detected by NMR.
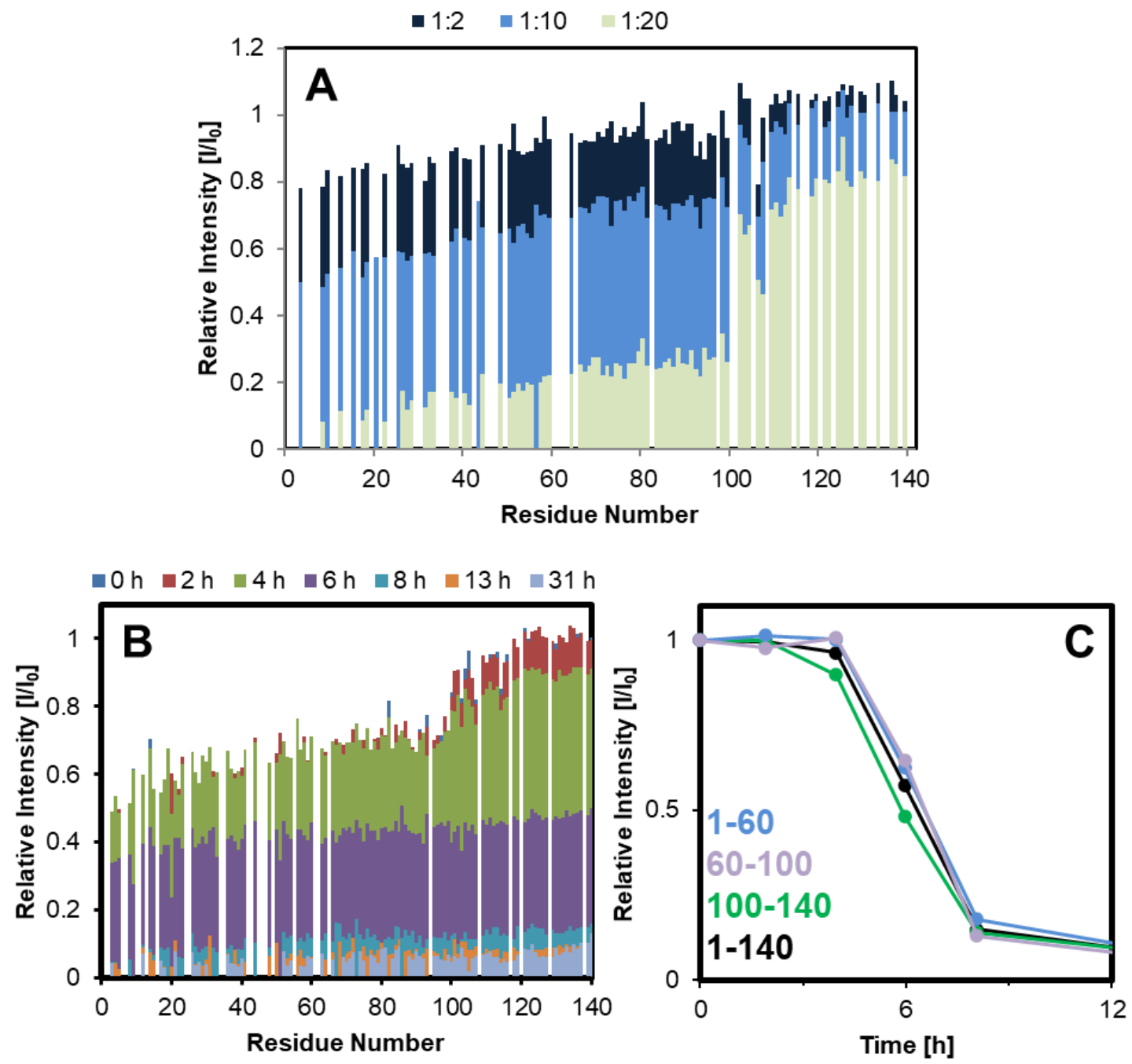

Figure 3.1.2: A) Titration of ${ }^{15} \mathrm{~N}$ - $\alpha$ S with SUVs of POPA and POPC (1:1) followed by ${ }^{1} \mathrm{H}-{ }^{15} \mathrm{~N}-\mathrm{HSQC}$; B) Intensities per residue of aS resonances in ${ }^{1} \mathrm{H}-{ }^{15} \mathrm{~N}-\mathrm{HSQC}$ during the aggregation of $\alpha S$ at $L / P=10$ under PMCA conditions; C) Development of relative Intensities ( $\left./ / l_{0}\right)$ derived from different parts of the $\alpha S$ sequence during aggregation.

It has to be emphasized, that unless one utilizes HSQC spectroscopy to resolve different domains of membrane bound $\alpha \mathrm{S}$, a slightly different curve for the development of monomer signal over time is obtained (Figure 3.1.2 C). As a result, the difference between monomer content derived from either C-terminus or full-length protein can be as much as 10 percentage points.

\subsubsection{Evidence for aS oligomers and an Effort at Quantification}

The relative intensities of the C-terminal domain obtained from ${ }^{1} \mathrm{H}-{ }^{15} \mathrm{~N}-\mathrm{HSQC}$ spectra were then plotted over time together with ThT intensities. For this purpose, fluorescence intensities were subjected to min-max normalization and adjusted by residual monomer concentrations 
preas outlined in chapter 7.5.9 of the methods section. The fundamental assumption was made, that at long aggregation times oligomers are non-existent and all unobservable monomers have been converted into fibrils. Since subtle disturbances originating from sample preparation and the stochasticity of the nucleation process largely influence the lag times $t_{\text {lag }}$ of amyloid aggregation, comparison of different experimental sets can be challenging. ${ }^{[145,146]}$ For this reason, the recorded kinetic data were referenced to an estimate of the lag time for the respective experiment comparable to previously reported procedures used for Lysozyme ${ }^{[147]}$ and microtubules. ${ }^{[61]}$ A plot of the monomer and fibril fractions over $t / t_{\text {lag }}$ from three independent aggregation experiments carried out under PMCA conditions is shown in Figure 3.1.3 A. Lag time estimation was performed on fluorescence data, so that experimental variation shows in the monomer data, while fibril data overlap reasonably well.

At $t /$ tlag $=0.8$, the monomer signal decreases sharply, dropping to about $50 \%$ of its initial

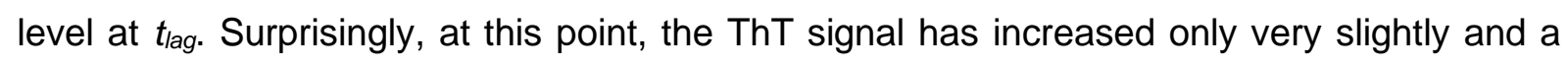
significant increase is only observed after tlag. Contrastingly, in the absence of lipids, fibril concentration mirrors monomer concentration (Figure 3.1.3 B), as decrease of the monomer signal at $t_{\text {lag }}$ is met with a proportional increase in ThT fluorescence. The decrease in monomer concentration without a concurrent increase in ThT fluorescence, suggests the formation of an aggregate species, such as oligomers or prefibrils, that can neither be detected by NMR due to its size or membrane association and is not yet sensitive to ThT, meaning that it has not yet fully formed the extended cross- $\beta$ structures characteristic to amyloid fibrils. A similar finding was made in a study by Iljina et al., when they investigated aS labeled with fluorophores by FRET analysis, detecting various oligomeric structures, lacking significant persistent structure and not binding to ThT. ${ }^{[148]}$ However, the observed levels of oligomer concentrations were much lower and the preparation method lacked phospholipids.

To probe, whether this precursive decrease in monomer concentration is specific to the presence of phospholipids, the experiment was repeated under orbital shaking conditions at $300 \mathrm{rpm}$ (Figure 3.1.3 C). However, both the decrease of monomer and the increase in ThT fluorescence start at $t_{\text {lag. }}$. It seems therefore, as if the observed behavior is unique to aggregation of aS aggregation in the presence of negatively charged phospholipids under PMCA conditions.

With fractions of both monomeric and fibrillary aS at hand, it was possible to derive the fraction of the oligomeric protein. Sigmoidal Dose Response fits were applied and restraints were implemented to achieve a good agreement with the experimental data in the initial phase of the aggregation process, to be able to derive any oligomer formation before 
formation of fibrils. During fitting, the top asymptote was fixed to the maximum fluorescence intensity, to assure, that fibril formation was not underestimated.
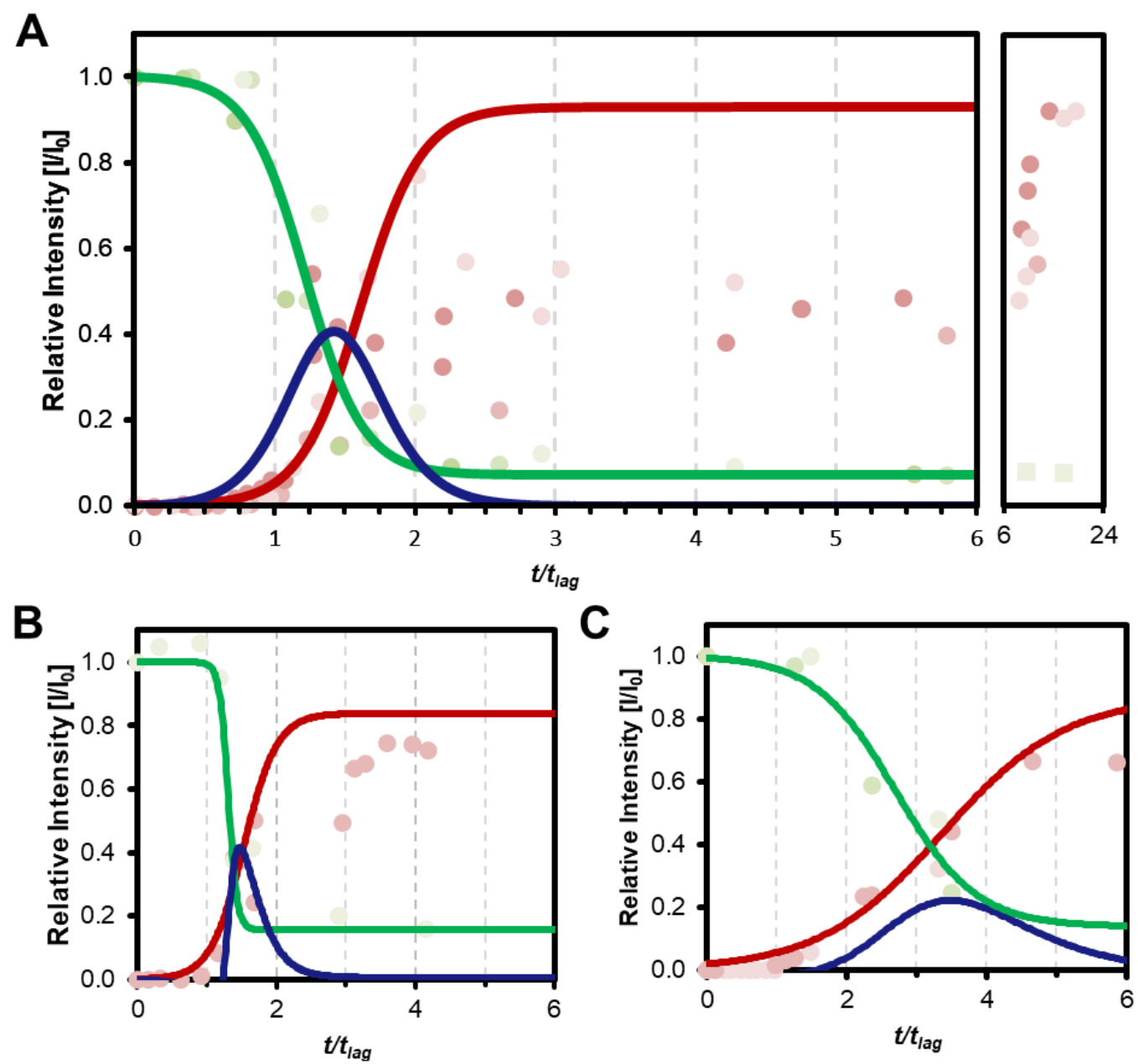

Figure 3.1.3: Aggregation of aS in the presence (A \& C) and absence (B) of phospholipids. A) \& B) PMCA, C) Shaking. ThT fluorescence (red) tracing fibrils and NMR spectroscopy tracing monomers (green - Res. 100-140 in ${ }^{1} \mathrm{H}-{ }^{15} \mathrm{~N}-\mathrm{HSQC}$ ); estimation of oligomer fraction (blue) was achieved by subtraction of the fitted curves.

As dose response fitting can only describe a flat plateau, fitting in the later stages of the aggregation process lacks quality, since the ThT fluorescence continues to rise. This is accepted in favor of a more accurate estimation of the oligomer fraction. The fraction of the oligomeric protein was derived as the difference of the fitted curves for monomers and fibrils. It should be stressed that these values should not be interpreted as absolute concentrations, but rather as qualitative evidence. The oligomer content is estimated to make up $20 \%$ of the total protein by $t_{\text {lag, so }}$ the point at which fibrillization starts to dominate. The maximum content at the intersection of monomer and fibril curve at $t / t_{\text {lag }}=1.5$ is estimated to be $40 \%$. In the absence of phospholipids, the oligomer content similarly reaches a maximum value of about $40 \%$, however no significant oligomer formation preceding fibril formation is detected. 
An analogous observation can be made in the case of shaking, although the estimated oligomer concentration peaks at $20 \%$.

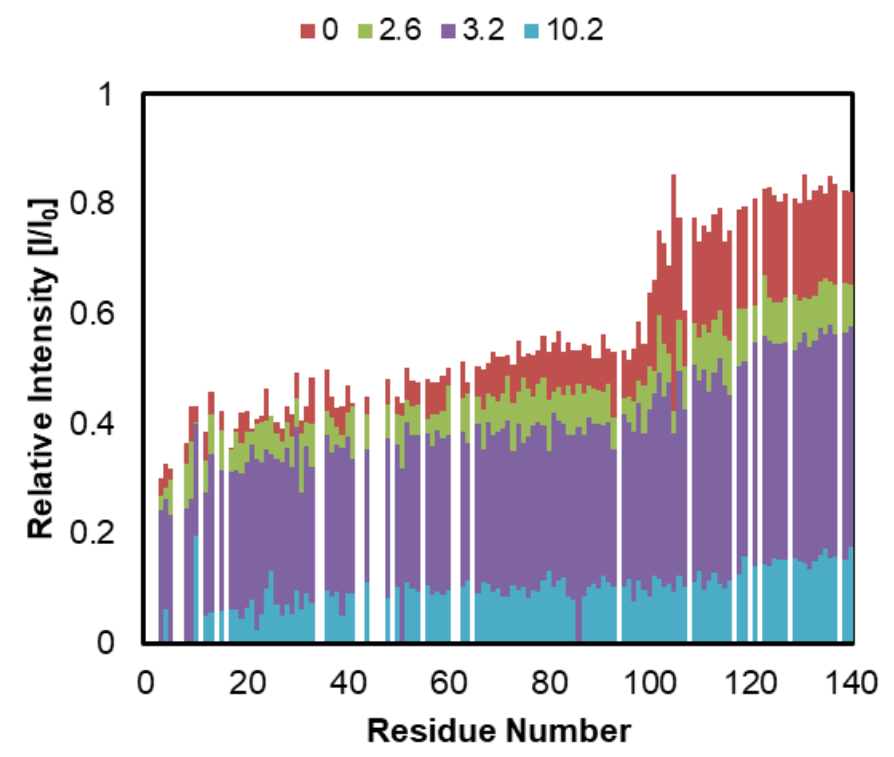

Figure 3.1.4: Intensities per residue of aS resonances in ${ }^{1} \mathrm{H}-{ }^{15} \mathrm{~N}-\mathrm{HSQC}$ during the aggregation of $\alpha \mathrm{S}$ at $L / P=10$ under orbital shaking conditions. Experiments are given in $t /$ tiag.

An inspection of the relative intensities derived from ${ }^{1} \mathrm{H}-{ }^{15} \mathrm{~N}-\mathrm{HQSC}$ spectra during $\alpha \mathrm{S}$ aggregation enhanced by orbital shaking, reveals retention of the attenuation profile characteristic to membrane binding until $t / t$ lag $\approx 3$ (Figure 3.1.4). This means that unlike under PMCA conditions, where this profile is lost after $t /$ tlag $\approx 1$, not all of the membrane associated $\alpha S$ has been converted into larger aggregates by the time fibrillization starts.

Further evidence of oligomer formation that precedes fibril formation is given by the development of the phospholipid resonances during aggregation. It was found for vesicles of POPA and POPC that at $t /$ tlag $=0.75$ the resonance intensity of the $\mathrm{CH}_{2}$ groups of the acyl chains had dropped to about $50 \%$ (Figure 3.1.5), while there was no increase in ThT fluorescence intensity. This finding is best described by the formation of a large membrane bound aggregate, that slows the tumbling of its associated vesicle, causing fast $\mathrm{T}_{2}$ relaxation. The same experiment performed in the presence of the purely zwitterionic POPC results in almost stable signals for the lipid resonances. Unless charge is present, aS does not associate with the vesicles so that aggregation proceeds like in the absence of lipids. At first the decrease in phospholipid signal intensity is accompanied by only a minor decrease in intensity of aS monomer signal, making this measurement highly sensitive to initial stage aggregation. 

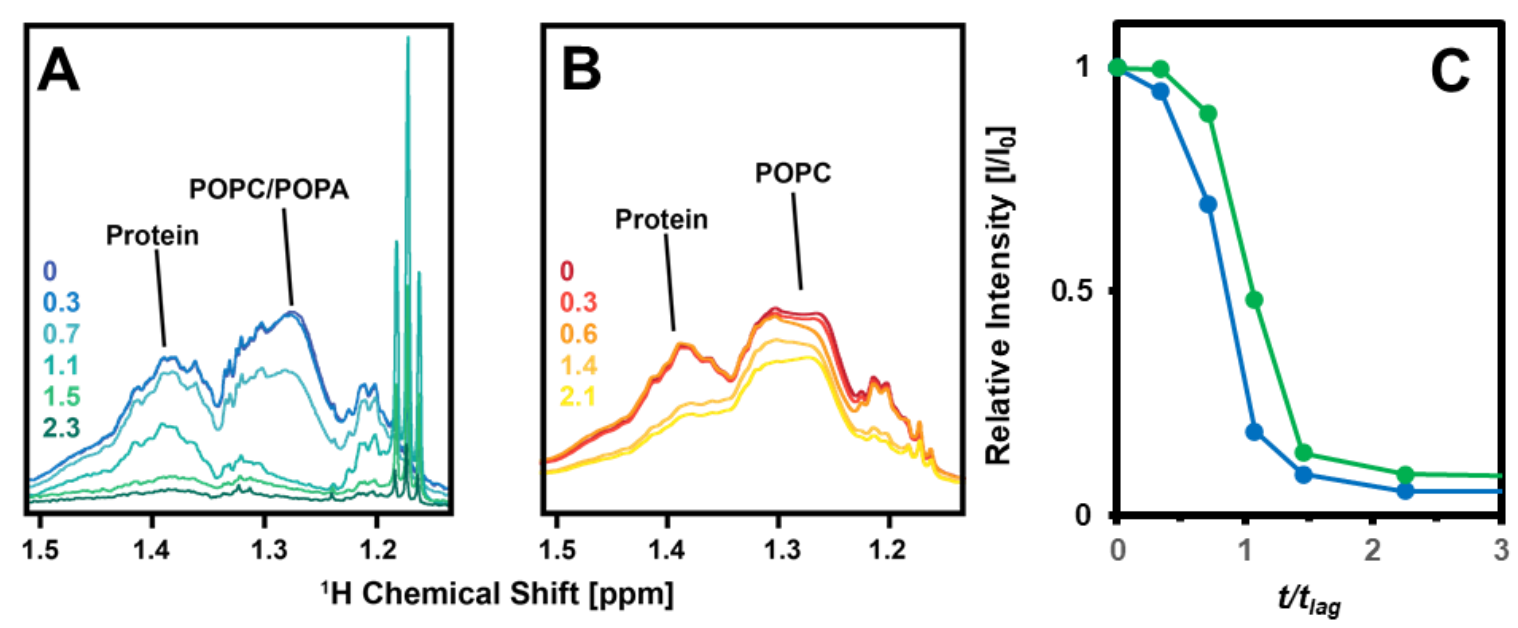

Figure 3.1.5: A) Decrease of resonances of POPC/POPA vesicles in a $1 \mathrm{D}{ }^{1} \mathrm{H}-\mathrm{NMR}$ spectrum during aggregation of aS Under the same conditions resonances of POPC remain mostly unchanged since aS does not interact with zwitterionic lipids; numbers indicate $t / t_{\text {lag }}$ at time of acquisistion. C) Development of relative intensities of POPC/POPA vesicles (blue) derived from 1D ${ }^{1} \mathrm{H}-\mathrm{NMR}$ and $\mathrm{aS}$ (green, $\left.{ }^{1} \mathrm{H}-{ }^{15} \mathrm{~N}-\mathrm{HSQC}\right)$. 


\subsubsection{Morphological Analysis of aS Oligomers by EM and AFM}

In order to complement the quantitative findings for oligomers with structural evidence, additional methods were employed to probe structural features of the species present during the individual stages of the aggregation process of aS in the presence of phospholipids under PMCA conditions.

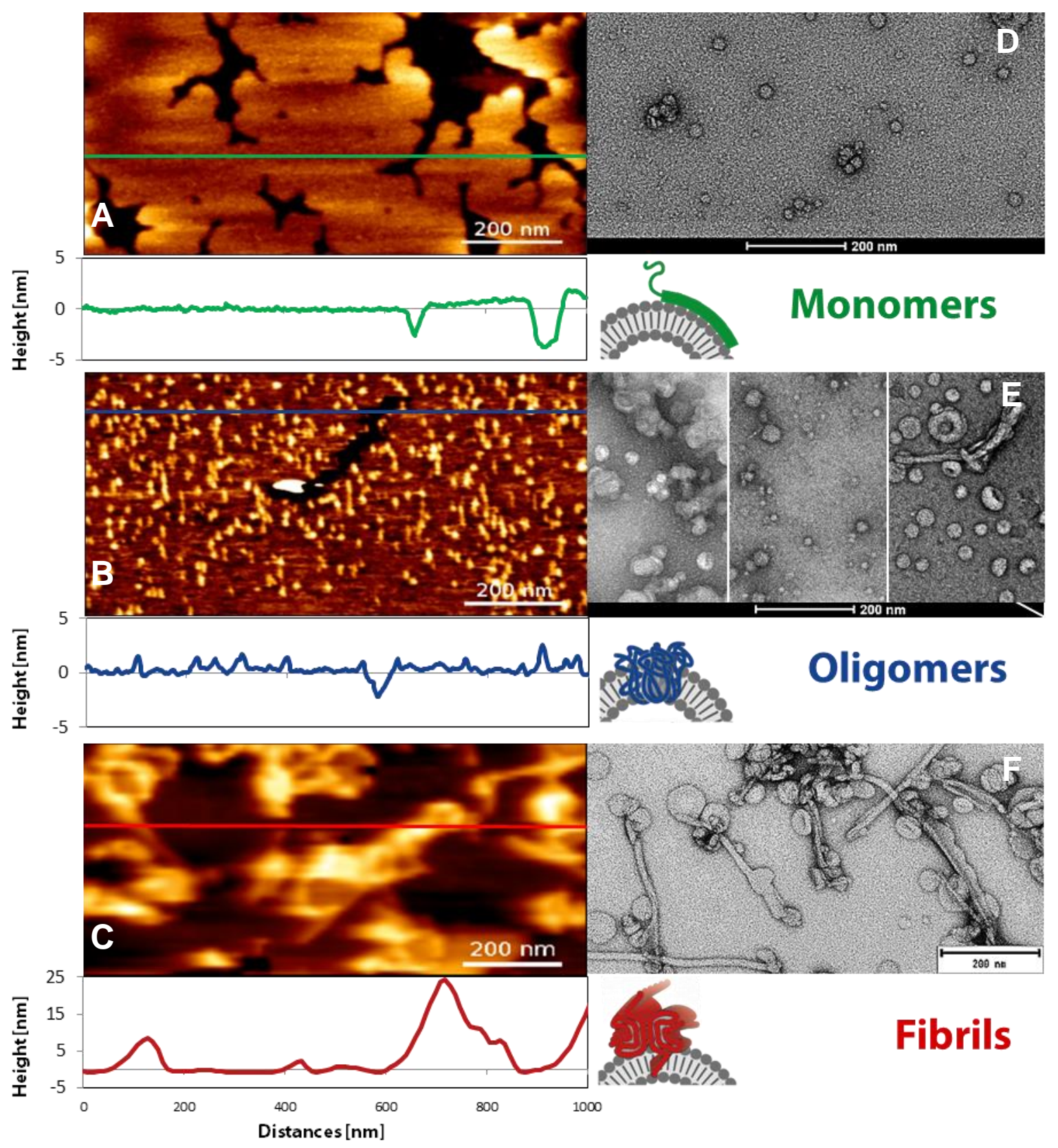

Figure 3.1.6: Species of aS observed during aggregation in presence of phospholipids Bottom AFM images with cross sections (left column) and EM images (right column) of samples at t/tlag $=0(\mathrm{~A}, \mathrm{D}), 1(\mathrm{~B}, \mathrm{E})$ and $\sim 3(\mathrm{C}, \mathrm{F})$. 
For morphological analysis by AFM, aliquots of aggregating samples were taken over time and incubated on mica and measured in liquid medium. Figure 3.1.6 shows height images of samples taken at characteristic points in time. Cross-sections through the height images confirm the formation of a supported lipid bilayer (SLB) on the mica support, indicated by characteristic height differences of $4-5 \mathrm{~nm}$. In the initial predominantly monomeric sample very few spherical shapes are detected, while most of the surface is covered in lipid (Figure 3.1.6 A). After about $5 \mathrm{~h}$ (Figure 3.1.6 B), very close to $t / t_{\text {lag }}=1$, a multitude of spherical and ellipsoidal shapes densely cover the SLB. These presumably oligomeric species exhibit diameters of $10-20 \mathrm{~nm}$ and heights of about $1-3 \mathrm{~nm}$, in agreement with larger aggregates embedded in the SLB previously reported. ${ }^{[149]}$ Images also show oligomers binding to patches of mica next to patches of bilayer analogous to results by Chadhaury et al.. ${ }^{[95]}$ Eventually, AFM images confirmed fibril formation (Figure 3.1.6 C), displaying short fragments of fibrils ranging from $100-200 \mathrm{~nm}$ in length and $5-25 \mathrm{~nm}$ in height, which is in line with previous reports. ${ }^{[89]}$ Fibril fragments often appear conglomerated together with lipid material, as structures are rather broad and globular patches of lipids seem to be spread on the mica surface alongside the protein structures.

These findings are corroborated by micrographs recorded by EM (Figure 3.1.6 D), showing SUVs of $20-50 \mathrm{~nm}$ in diameter as the dominant initial species. The size and shape of the oligomeric species found by AFM is confirmed by the appearance of spherical particles with a diameter below $20 \mathrm{~nm}$ (Figure 3.1.6 E), which is not justified by micelle formation, as the lipids utilized here have been shown to exhibit a minimum vesicle diameter larger than this. ${ }^{[150,151]}$ Alongside these smaller particles vesicles often clot and seem to be disrupted. Additionally, micrographs display minor amounts of short fibrils of mostly 100-200 nm, but up to $400 \mathrm{~nm}$ in length, which are bound to lipid vesicles along their whole surface. Upon long PMCA incubation times these lipids appear to rupture and eventually cover the fibril surface in a fashion resembling the processes during SLB formation (Figure 3.1.6 F). ${ }^{[152]}$ Vesicle rupturing might be augmented due to repeated sonication cycles during PMCA and might in return explain the slightly higher fibril thickness of about $15 \mathrm{~nm}$. 


\subsubsection{Evolution of Secondary Structure during aS aggregation}

Secondary structure information was gathered by CD-Spectroscopy over the whole course of the aggregation process, as has been done for NMR-Spectroscopy. CD-Spectra of aliquots taken from the reaction mixture are displayed in Figure 3.1.7 for samples in both the presence and absence of phospholipids. Due to a drop off in light flux from the Xenon lamp, ellipticities acquired in the range from $180 \mathrm{~nm}$ to $190 \mathrm{~nm}$ are not included. This problem is commonly known for commercial CD instruments and causes the values in this range to be invalid. ${ }^{[153]}$ This problem is amplified by residual $\mathrm{NaCl}$ from the reaction mixture and an increased turbidity upon aggregation. Before the start of aggregation, a decreased ellipticity at $222 \mathrm{~nm}$ in the presence of phospholipids hints to an a-helical membrane bound state. ${ }^{[109]}$ Nevertheless, the large negative peak at $200 \mathrm{~nm}$ indicates, the structure of the protein in both cases is predominantly disordered. ${ }^{[153]}$ Upon aggregation this peak aggregates and eventually disappears, as the more ordered aggregate become the dominant species in the reaction mixture.

In the presence of lipids, at $t_{\text {lag }}$ the peak at $200 \mathrm{~nm}$ decreases and shifts to about $202 \mathrm{~nm}$, while simultaneously the ellipticity shows a decrease at $217 \mathrm{~nm}$. This behavior is typical for $\beta$-sheet formation and agrees with the increase of ThT fluorescence from this point on. The spectra of samples after $t / t_{\text {lag }}$ show drastically different features, displaying a broad peak centered at $217 \mathrm{~nm}$. This spectrum is characteristic to the parallel $\beta$-sheet of amyloid fibrils. ${ }^{[154]}$ When comparing this spectrum to the one at $31 \mathrm{~h}$, it is obvious, that the structural rearrangement is still incomplete, indicated by a shoulder at $205 \mathrm{~nm}$.
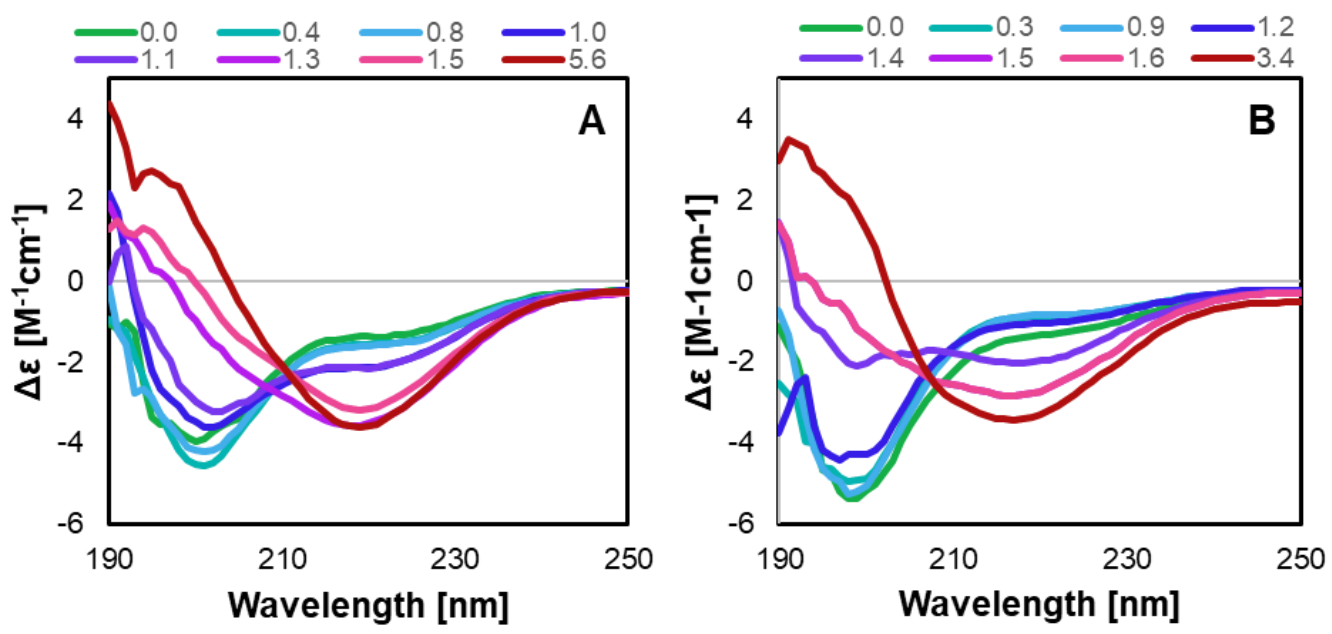

Figure 3.1.7 CD Spectra of $\alpha S$ in the presence $(A)$ and absence $(B)$ of phospholipids during the aggregation under PMCA conditions 
In the absence of lipids, at $t_{\text {lag }}$ only a slight decrease of the peak at $200 \mathrm{~nm}$ has appeared, with little change at $215 \mathrm{~nm}$. This spectrum stands in stark contrast to the one at $12 \mathrm{~h}$, which features a small negative peak, both at 217 and $202 \mathrm{~nm}$, showing the rapid accumulation of $\beta$-sheet content. The transition from disordered to ordered hence seems less gradual, albeit for a missing spectrum at $11 \mathrm{~h}$, which was not recorded and seems to withhold crucial piece of information on the actual process. After this major structural arrangement, spectra are comparable to those in the presence of lipids, showing a broad negative peak at $217 \mathrm{~nm}$.

Because CD-spectra are linear combinations of the basis spectra, the analysis of the CDspectra yields only a general idea of the contents of $\alpha$-helical, $\beta$-sheet and disordered regions. Modern algorithms however are able to deconvolute such spectra and give a quantification of the structural elements in the species under investigation. ${ }^{[155]}$ For this purpose, the BeStSel algorithm (Beta Structure Selection) was used, which is accessible as an online tool by courtesy of the group of Jósef Kardos. ${ }^{[156]}$ The potency of this algorithm has been shown in earlier publications. ${ }^{[154,155]}$
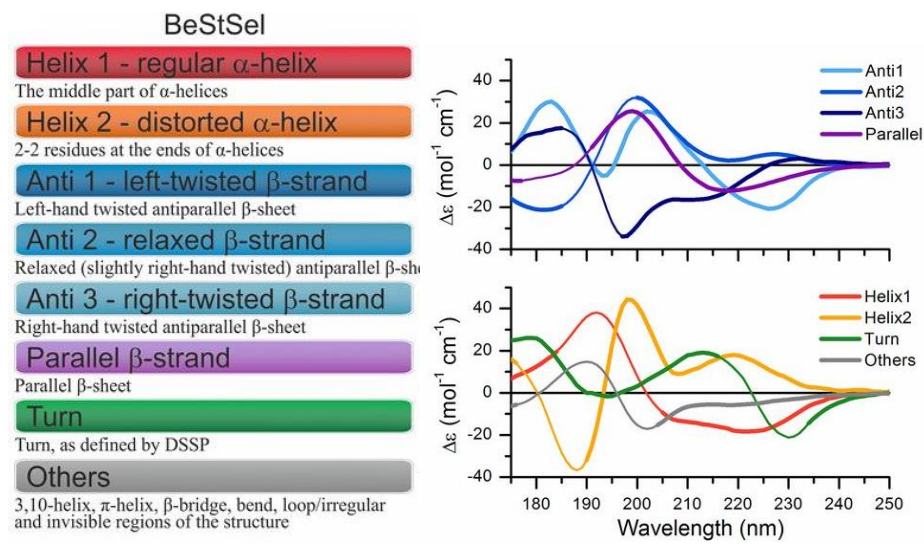

Figure 3.1.8: A) Classes of secondary structures defined by BeStSel for CD Data deconvolution, B) Typical CD spectra for the secondary structures listed in A) [adopted from Micsonai et al. PNAS 2015]

Apart from just quantifying the secondary structure, it also classifies the structural component. Helical parts are distinguished into regular and distorted helix. The unique feature however is the ability to distinguish not only the orientation of neighboring $\beta$-strands (parallel vs antiparallel), but also the twist of antiparallel $\beta$-sheets. The structural classes used by the algorithm are shown in Figure 3.1.8 A. The characteristic curves of these structural elements exhibit significant differences in the in the region between 175-190 nm, so that the data used here will be inherently susceptible to bias for reasons outlined above.

Secondary Structure calculation was performed on the individual spectra in Figure 3.1.7 in the region 190-250 nm, yielding a time course of the development of the structural features. Besides a content of about $50 \%$ disordered structure, the initial monomeric samples in the 
absence of phospholipids (Figure 3.1.5) are predicted to contain about $17 \%$ hydrogen bonded turns, about $6 \%$ helical elements and most prominently $28 \%$ of anti-parallel $\beta$-sheet.
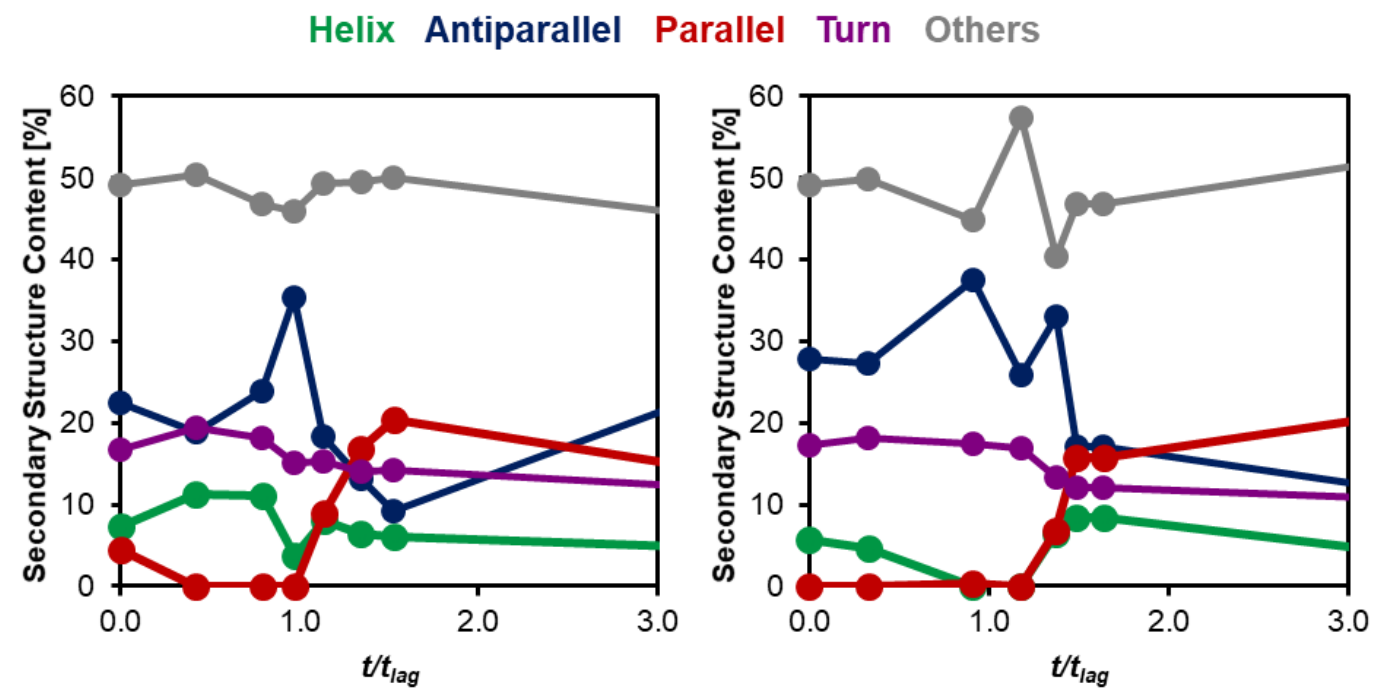

Figure 3.1.9 : Development of secondary structure content of $\alpha S$ in the presence $(A)$ and absence (B) of lipids during the aggregation under PMCA conditions; Secondary Structure was calculated by BeStSel (http://bestsel.elte.hu/).

This amount of secondary structure is quite remarkable for an IDP, although it is very likely due to minor differences in the regime between $190-200 \mathrm{~nm}$ and the close resemblance of spectra from right twisted $\beta$-strand, to those of disordered proteins. Nevertheless, $\alpha$-synuclein has been shown to possess a tertiary structure and exhibit long range contacts between $\mathrm{N}$ and C-terminus, that might at least account for a small amount of the observed structural

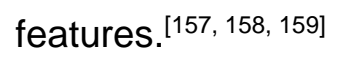

In the presence of phospholipids the helical content of the sample is increased to about $8 \%$, which seems small, considering that attenuation of signals in the $\mathrm{N}$-terminal domain recorded by ${ }^{1} \mathrm{H}-{ }^{15} \mathrm{~N}-\mathrm{HSQC}$ ranges from $30-40 \%$, for the present L/P ratio of 10 (Figure 3.1.2). In the same sample a small amount of $\beta$-sheet is detected (Figure 3.1.9 A), in contrast to ThT experiments.

Most notably, at $t_{\text {lag, }}$ in the presence of phospholipids, a sharp increase in anti-parallel $\beta$ sheet content up to $35 \%$ is detected. The sudden decrease at the same time for lipid free $\alpha \mathrm{S}$ is caused by poor fitting, due to an artifact in the spectrum. This finding supports the idea of an oligomer formed during the aggregation under PMCA conditions, as ThT is only sensitive to parallel in register $\beta$-sheets. Such $\alpha \mathrm{S}$ oligomers containing anti-parallel $\beta$-sheets have been previously reported by use of FTIR-spectroscopy. ${ }^{[89,160]}$ Upon full aggregation, a high parallel $\beta$-sheet content would be expected, however fitting severely hampered by artifacts originating from increased turbidity in those samples. This problem is only partially accounted for by reducing the input data to the range $200-250 \mathrm{~nm}$. 
Since CD spectra were acquired on unpurified mixtures of various protein species, it is crucial to ask the question, whether spectra of the presumed anti-parallel oligomer are simply a superposition of the initial monomer and final fibril spectra. Averaging of the latter spectra in the presence of lipids yields a spectrum that does not overlap with the spectrum at $t_{\text {lag }}$ (Figure 3.1.10 A), showing a broad peak around 217, with a shoulder around $205 \mathrm{~nm}$, similar to spectra observed at later stages in the aggregation process. Deconvolution of these spectra yields a parallel $\beta$-sheet content of about $14 \%$ and a helical content of about $5 \%$, which confirms the conformations that are expected from the input spectra.
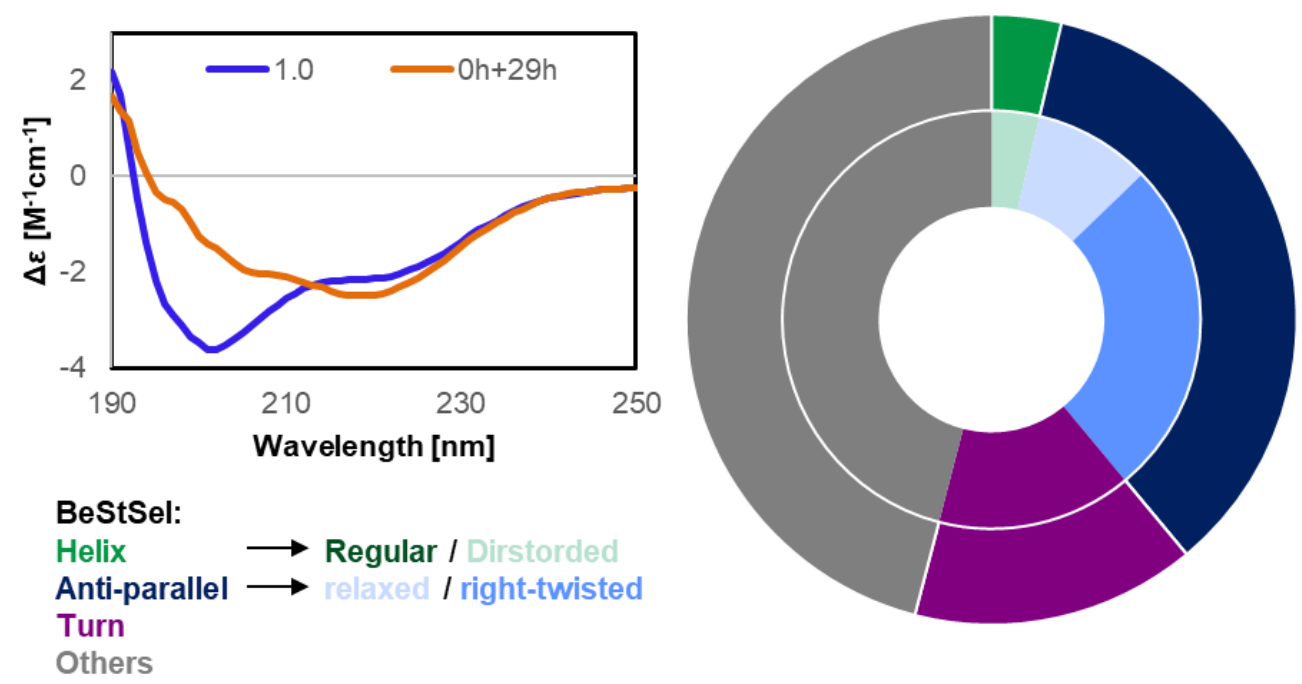

Figure 3.1.10: A) Comparison of the CD-spectrum at $t_{\text {lag }}$ (35\% anti-parallel $\beta$-sheet) and a superposition of the first sample (monomer) and last sample (fibril); B) Donut diagram of the secondary structure contents of the sample at tag; outer shell: Secondary structure content, inner shell: subtypes as found by BeStSel.

Exploiting the full potential of the BeStSel algorithm, its ability to calculate the twist of $\beta$-sheet and characterize the type of helix was used (Figure 3.1.10 B). The helical part is estimated to be predominantly distorted, with a ratio of $4.5: 1$ over a regular $\alpha$-helix. A possible explanation might be the interaction with the highly curved membrane of the SUVs in the sample, which causes the deviation from regular helix spectra. The anti-parallel $\beta$-sheet is composed of $9 \%$ relaxed (twist angle of $0^{\circ}$ ) and $26 \%$ right-twisted strands. It has to be emphasized, that both these components are non-zero at the start of the aggregation process and it is the increase in the anti-parallel $\beta$-sheet content of 15 percentage points that should be considered as the potential oligomer component. As outlined earlier, there will be contributions from other species to these spectra, especially from monomer, which has decreased to $50 \%$ at this stage (as traced by NMR). The structural implications and potential topologies that can be derived from these findings are discussed at a later point. 


\subsection{Characterization of aS fibrils and intermediates by ssNMR}

\subsubsection{Assignments of $\alpha \mathrm{S}$ fibrils in the presence of phosplipids}

Building on the previously acquired knowledge on the kinetics of aS aggregation, samples of aggregates prepared by PMCA were characterized by solid-state NMR. Despite recent advances in structural characterization of aS fibrils and oligomers, studies on aggregates grown in the presence of phospholipids are very limited. ${ }^{[91]}$ The clear indication of a membrane association of the aggregation relevant species led to the choice of ultracentrifugation as means of enriching the respective aggregate species. Samples were not lyophilized to avoid potential disturbances or changes of lipid protein interactions through removal of water molecules. Furthermore, Comellas et al. found, that hydrated aggregate samples are preferable to their dry counterparts, owing to improved linewidths and increased sensitivity, which outweigh decrease in CP enhancement. ${ }^{[161]}$

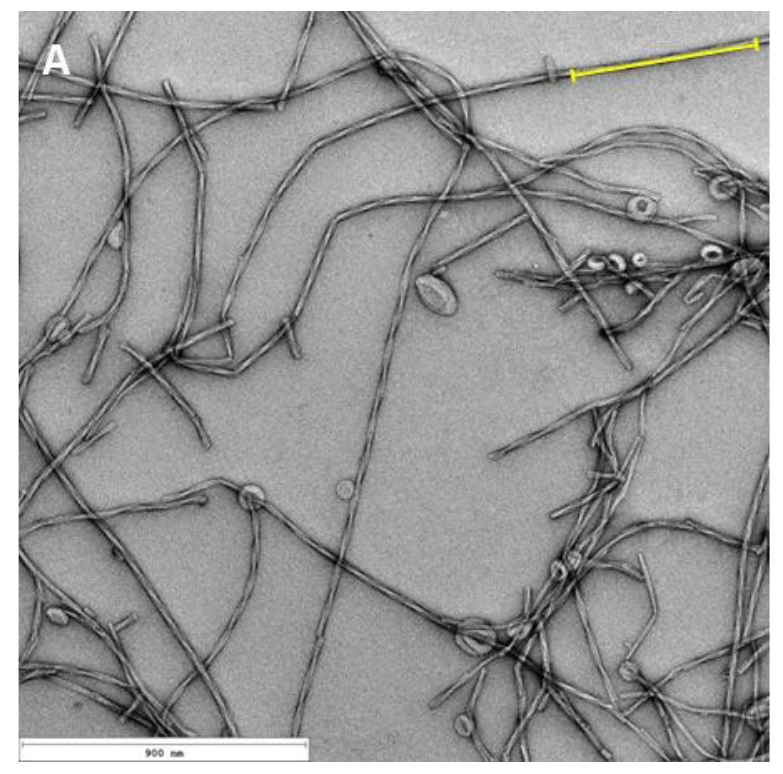

B

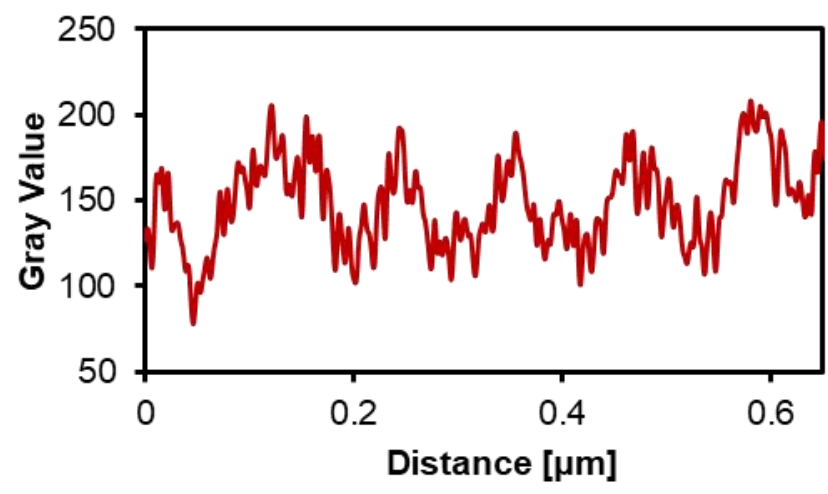

\begin{tabular}{|l|l|l|l|l|}
\hline Appearance & Length & Helical pitch & Thickness & Prevalence \\
\hline Highly Twisted & $50-100 \mathrm{~nm}$ up to 1 $\mathrm{m}$ & $80-120 \mathrm{~nm}$ & $10-15 \mathrm{~nm}$ & Dominant \\
\hline Long twist & $0.5-1 \mu \mathrm{m}$ & $150-160 \mathrm{~nm}$ & $10-15 \mathrm{~nm}$ & Rare \\
\hline $\begin{array}{l}\text { Single } \\
\text { stranded }\end{array}$ & $\begin{array}{l}\text { Mostly short, below } \\
0.5 \mu \mathrm{m}\end{array}$ & & $5-7 \mathrm{~nm}$ & $\begin{array}{l}\text { Higher abundance in } \\
\text { late stage }\end{array}$ \\
\hline
\end{tabular}

Figure 3.2.1: A) TEM images aS fibrils in the presence of phospholipids prepared by PMCA. B) Profile plot across a representative fibril indicated by the yellow line in A. Table: structural properties of fibrils as observed in TEM images 
Characterization of the fibril samples by TEM yielded three major types of fibril as identified by their coarse structural features:

1. High twist two strand fibrils

2. Low twist two strand fibrils

3. Single strand fibrils

The observed helical pitch of $80-100 \mathrm{~nm}$ is in good agreement with literature. ${ }^{[101]}$ Due to the high order of symmetry of the structure, a fibril composed of two filaments can most likely not be distinguished by NMR spectroscopic methods from a single filament. Further, the minor changes in the local atomic environment due to a difference in helical pitch are not expected to produce major chemical shift changes, as was confirmed earlier. ${ }^{[162]}$ The repeated sonication in the fibril preparation breaks them into short fragments, so that little information can be derived from their length.

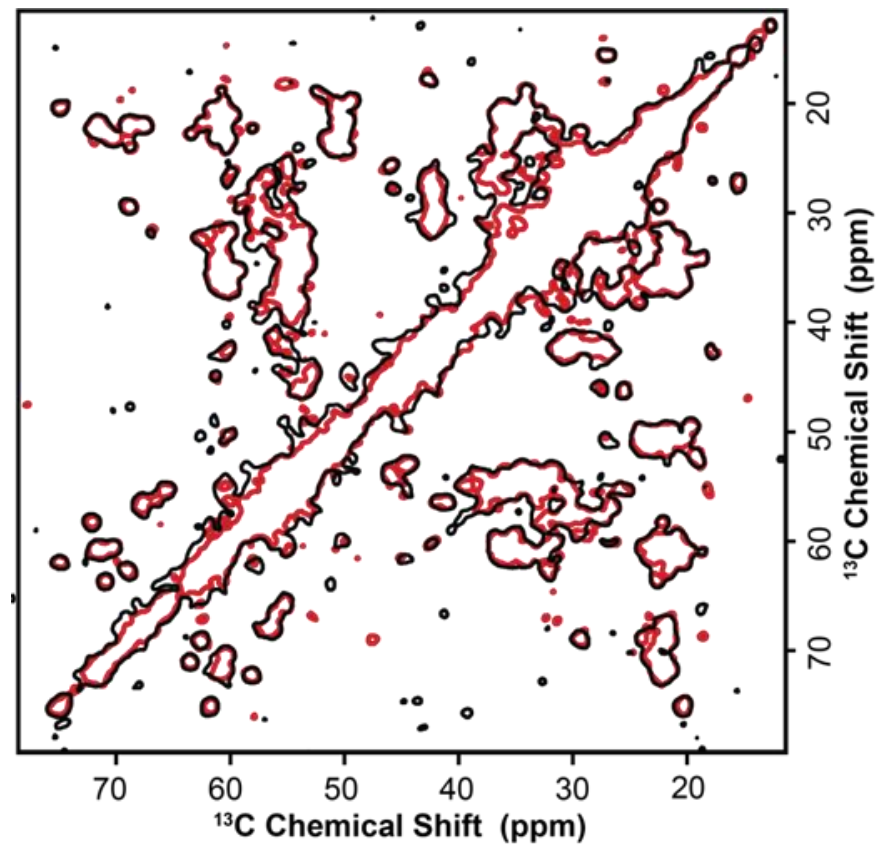

Figure 3.2.2: ${ }^{13} \mathrm{C}-{ }^{13} \mathrm{C}$-DARR Spectra with $20 \mathrm{~ms}$ mixing time of two identical batches of $U{ }^{13} \mathrm{C}$ aS fibrils in the presence of phospholipids.

Since fibrils were generated de novo without the use of seed material, reproducibility is a major concern, as there is no apparent template effect in the preparation at hand. Nevertheless, ${ }^{13} \mathrm{C}-{ }^{13} \mathrm{C} 2 \mathrm{D}$ correlation spectra using dipolar assisted rotational resonance (DARR) mixing on uniform $(U){ }^{13} \mathrm{C},{ }^{15} \mathrm{~N}$ aS fibrils in the presence of POPA and POPC displayed no obvious differences between the batches prepared in this work (Figure 3.2.2).

In order to gain insight on the conformational landscape of the fibrils, sequence specific assignment of $\alpha \mathrm{S}$ fibrils in the presence of phospholipids was conducted. For this purpose, we recorded proton detected hCANH, hcoCAcoNH, hcaCBcaNH, hcaCBcacoNH, hcaCONH 
and hCONH ssNMR spectra( Figure 3.2.4: 13C-13C correlation spectrum with DARR mixing of $20 \mathrm{~ms}$ of $\mathrm{U}-13 \mathrm{C}-15 \mathrm{~N}$ - $\alpha \mathrm{S}$ fibrils with assignments. Low confidence assignments are labelled in gray.). Experimental details and explanations of the spectra are listed in Chapter 7.5.12. Resonance assignments, based on these spectra were started, but could not be finished in the course of this work. Nevertrheless assignments for residues 50-94 were obtained (Figure 3.2.4).

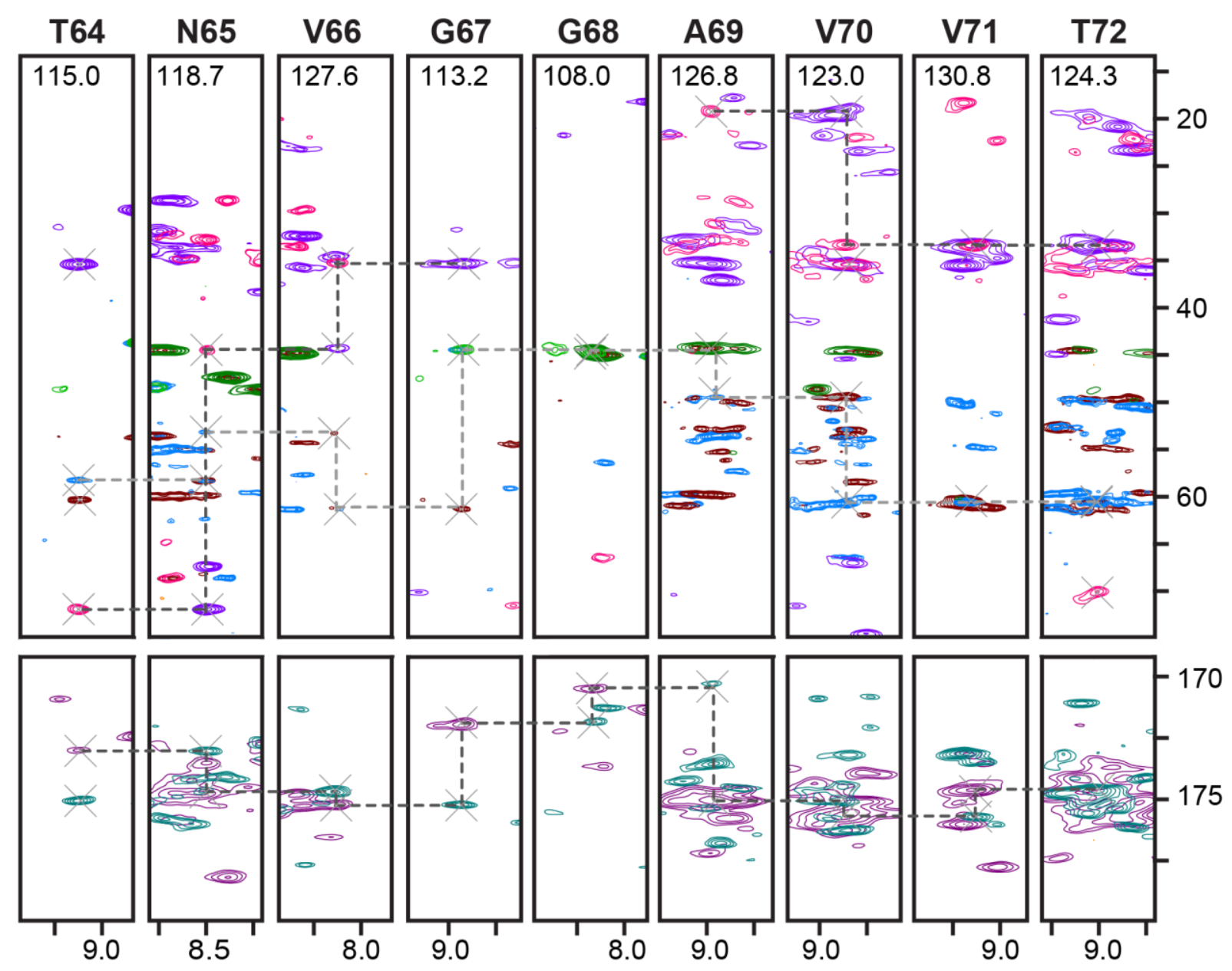

Figure 3.2.3: Strip plot for a generic sequence walk along ${ }_{64} \mathrm{TNVGGAVVT}_{72}$ in proton-detected $3 \mathrm{D}$ Spectra. Chemical shift differences between BMRB 18860 and the polymorph prepared under PMCA conditions in the presence of lipids start at residue 70. Top: Aliphatic Carbons: blue: hCANH, brown: hcoCAcoNH, red/light green: hcaCBcaNH; purple/green: hcaCBcacoNH; Bottom: Carbonyl Carbons: purple: hcaCONH, teal: hCONH.

Analysis of chemical shift data by TALOS + revealed several stretches of interrupted $\beta$ sheets, as previously found in other studies. ${ }^{[163,164]}$ Furthermore, two sharp turns are found, for residues V74 and T75 as well as K80 and T81, indicated by their helical propensity. A loop is found for residues the sequence ${ }_{58} \mathrm{KTKE}_{61}$, although with low confidence values. The turns are confirmed by CSI 3.0, complemented by a putative $\beta$-hairpin within residues $50-77$. The suggested hairpin supports the idea of a turn at residues T59 and K60. 


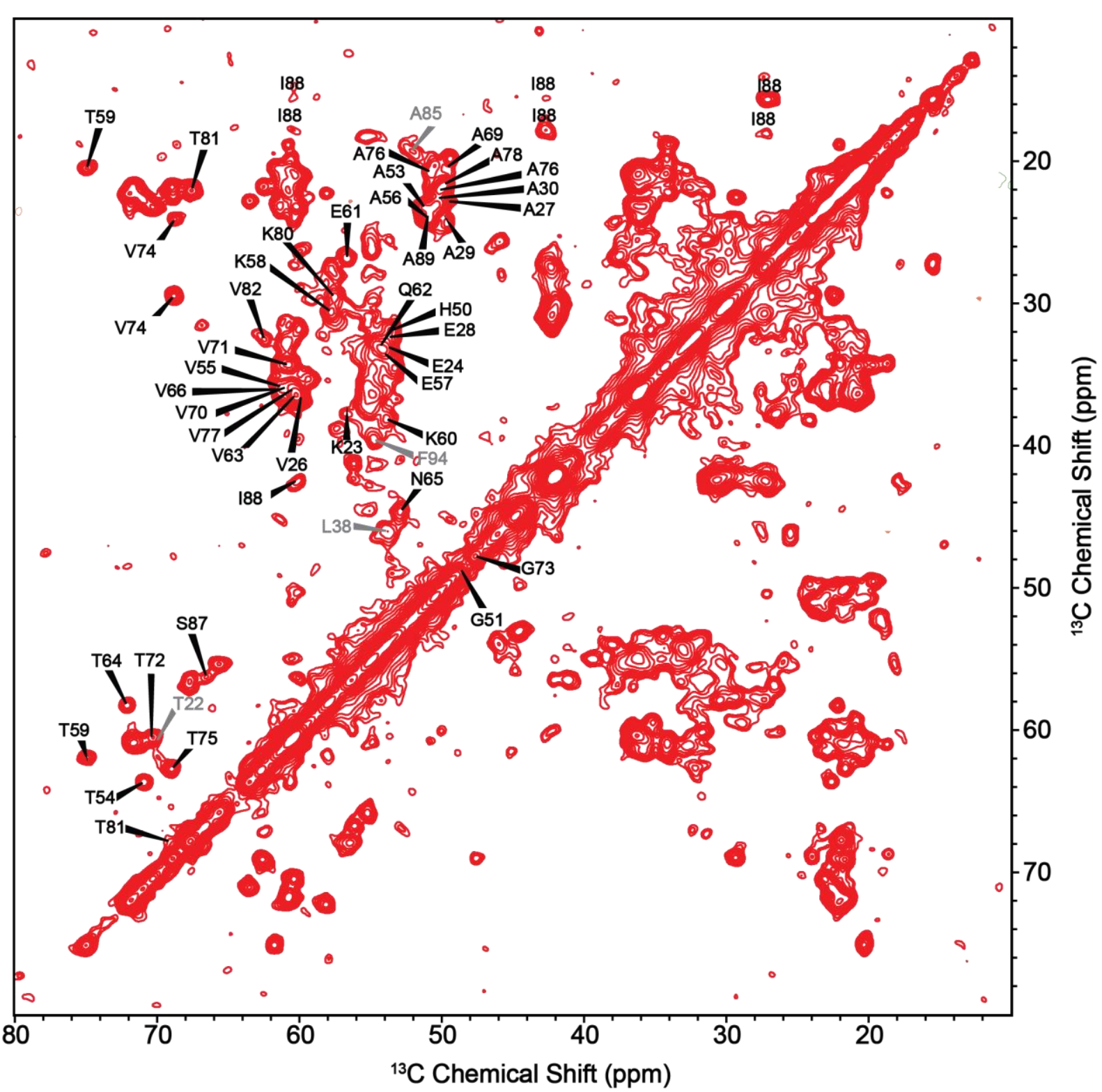

Figure 3.2.4: ${ }^{13} \mathrm{C}-{ }^{13} \mathrm{C}$ correlation spectrum with DARR mixing of $20 \mathrm{~ms}$ of $\mathrm{U}-{ }^{13} \mathrm{C}-{ }^{15} \mathrm{~N}-\alpha \mathrm{S}$ fibrils with assignments. Low confidence assignments are labelled in gray.

Due to the lack of detailed atomic resolution structural information, the recorded 2D spectra were compared to spectra published on two of the three structurally characterized polymorphs. Adopting the nomenclature from Guerrero-Ferreira et al. these polymorphs are referred to as polymorph 2 and Polymorph 1a, as depicted in Figure 1.3.3. ${ }^{[100]}$ According to the authors polymorphs $2 \mathrm{a}$ and $2 \mathrm{~b}$ are indistinguishable by ssNMR, so that they are treated as a single species in the following analysis. Up to this date there are no published NMR spectroscopic data on polymorph $1 \mathrm{~b}$, so that they are excluded from the discussion. Despite this caveat, the structural similarities of large parts of the structure of polymorphs $1 \mathrm{a}$ and $1 \mathrm{~b}$ are expected to produce at least comparable spectra. ${ }^{[99]}$ For the sake of simplicity they are hence referred to as polymorph 1. 


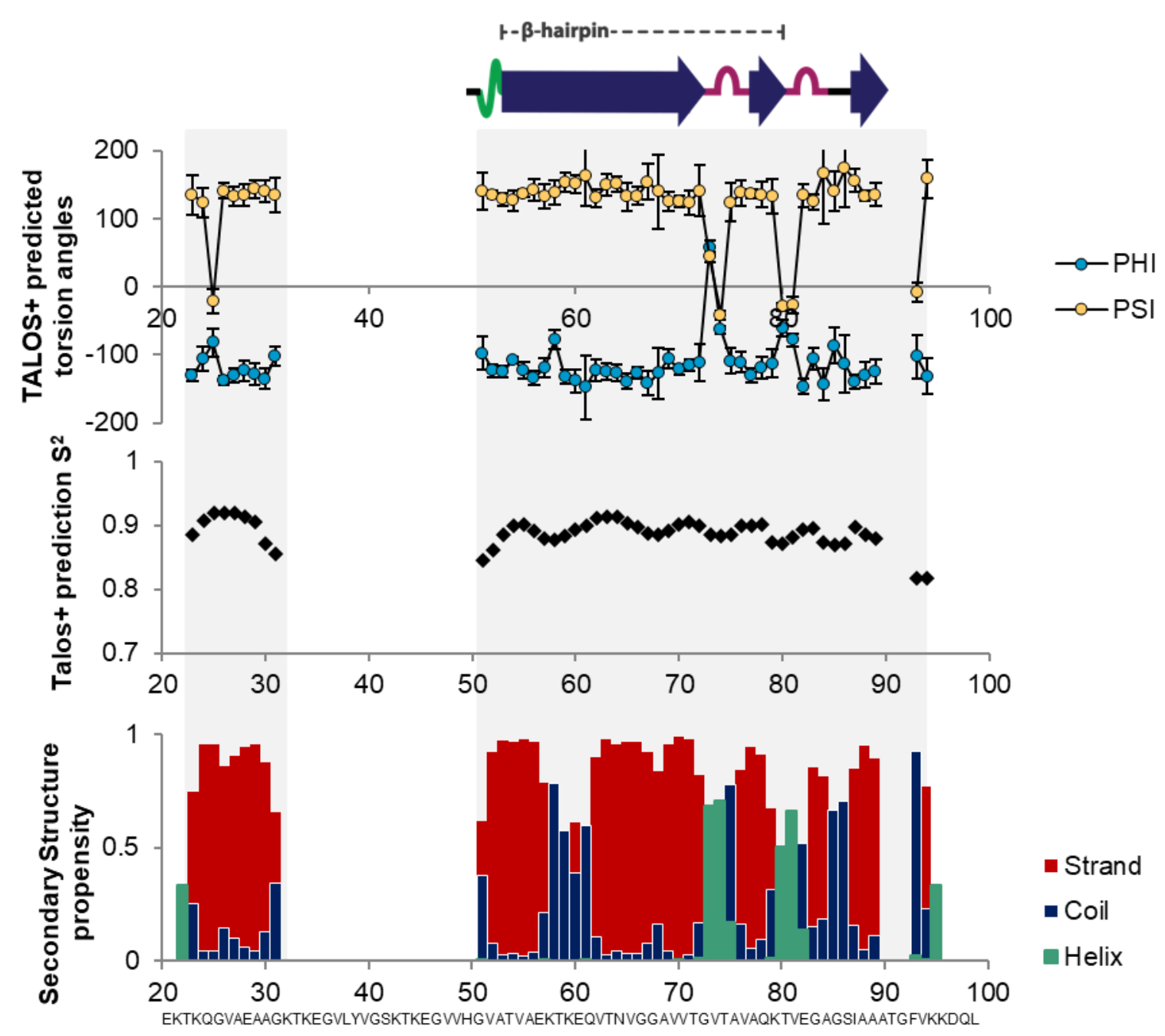

Figure 3.2.5: Torsion angles (top), prediction $S^{2}$ (center) and secondary structure propensity (bottom) derived by TALOS+ from $C_{\alpha}, C_{\beta}$ and $C^{\prime}$ chemical shifts of aS fibrils; Cartoon: Secondary structure prediction by CSI 3.0 for the region $51-87$.

Experimental data were overlaid with the published ${ }^{13} \mathrm{C}-{ }^{13} \mathrm{C} 2 \mathrm{D}$ spectra with DARR mixing for polymorph $1^{[165]}$ and polymorph $2^{[163]}$ (Figure 3.2.6).. In the analysis of several literature spectra alongside the ones reported here, the Threonine residues, especially the $C_{\alpha}-C_{\beta}$ correlation were found to be very characteristic to polymorphs and can almost be treated as a fingerprint. Chemical shift perturbations of $C_{\alpha}$ and $C_{\beta}$ were subtracted from the published data of polymorph 2 (BMRB Entry 18860), to quantify potential similarities (Figure 3.2.6). The comparison revealed the sequence from V70-K80 with the turn at V74 and T75 as a common motif of the two structures. Since assignments of residues H50-K32 was not achieved, little can be said about their geometry, however, given the expected loop for K70$\mathrm{K} 80$, they are likely to resemble their counterparts in polymorph 2. Major chemical shift differences were found for the loop region formed by T54-A69, especially for residues K58, T59, K60 and E61, which are likely to form the kink in between two straight segments. 

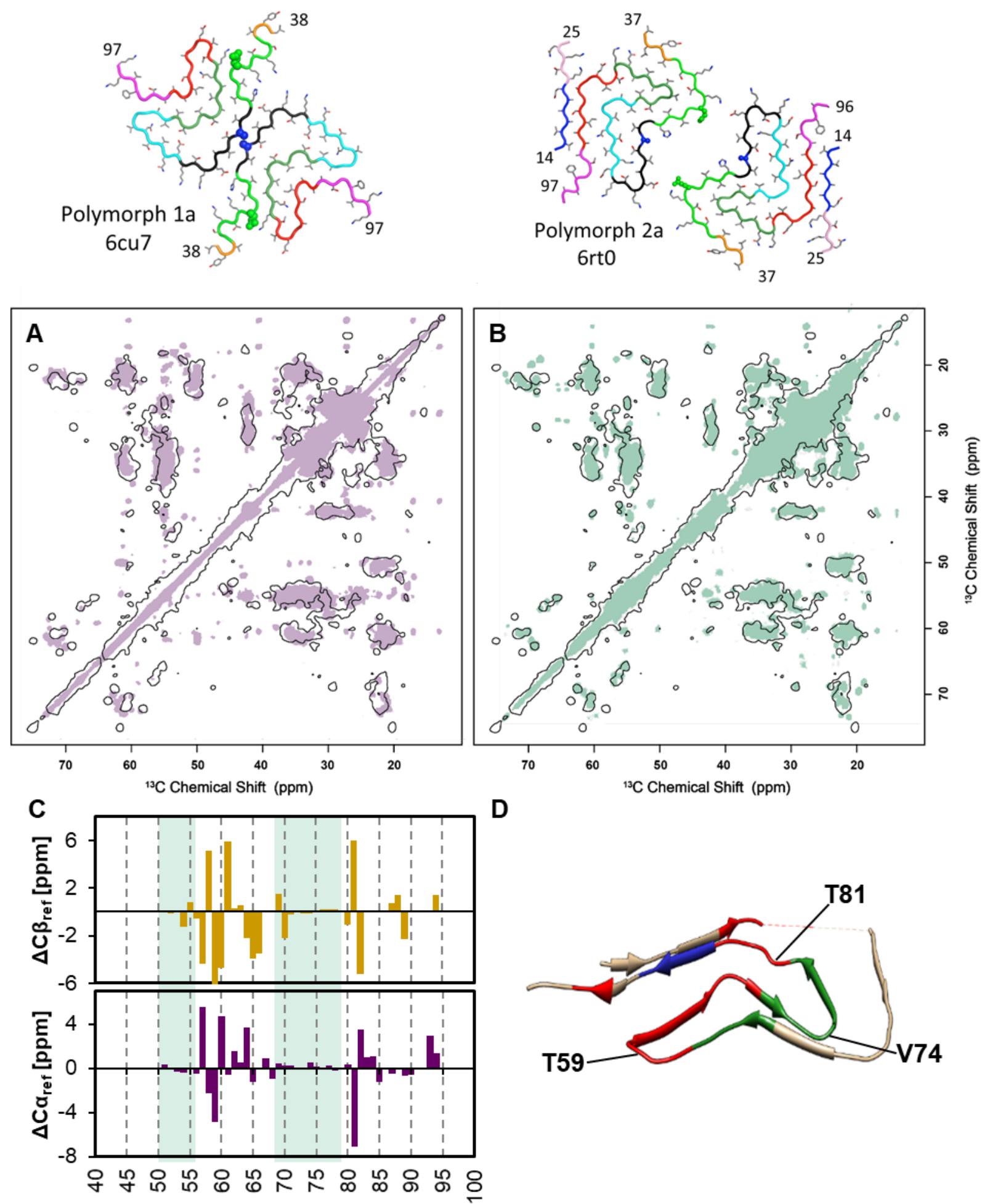

D

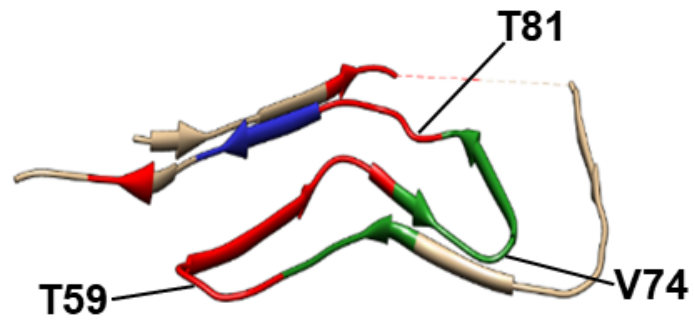

Figure 3.2.6: A) Comparison DARR Spectra of aS fibrils in this work (black outline) with polymorph 1 (A, purple - BMRB 25520) and polymorph 2 (B, green - BMRB 18860), C) Chemical shift differences compared to BMRB 18860; D) Chemical shift differences mapped onto PDB structure 6rt0 of polymorph 2, green areas show agreement, red areas differences to 18860 , blue area shows partial agreement.

Further distinctions are seen for residues T81-F94, for which the sequence was not continuously assigned, but particularly T81 and V82 seem to adopt a markedly different conformation than in the published polymorph, showing a sharp turn predicted by TALOS+. 
Surprisingly, resonances for an extended stretch of residues from T22-G31 are observed, which are unstructured in the two structures characterized by cryo-EM. This region was also identified by Gath et al. in an earlier study using ssNMR, albeit with different chemical shifts. ${ }^{[166]}$ In their initial NMR-spectroscopic study on polymorph 2 the authors observed a significant amount of unassignable peaks, which they attributed to residues 1-37, although this was only partially supported by further studies. ${ }^{[163]}$

In conclusion the polymorph prepared by PMCA in the presence of phospholipids shares major structural features with the previously published polymorph 2 , however certain arrangements of residues, especially in the turns at T59 and T81 seem to be significantly different. 


\subsection{2 $\alpha \mathrm{S}$ intermediates in the presence of phospholipids}

In order to gain structural information on the oligomers observed in chapter 3.1 and to identify a possible structural influence of anle138b, samples at various characteristic points in time of the aggregation were characterized by ssNMR. For this purpose, ${ }^{13} \mathrm{C}-{ }^{13} \mathrm{C} 2 \mathrm{D}$ correlation spectra with DARR mixing of $U{ }^{13} \mathrm{C}$ aS incubated under PMCA conditions in the presence of vesicles of POPA and POPC containing anle138b were acquired (Figure 3.2.7 A).
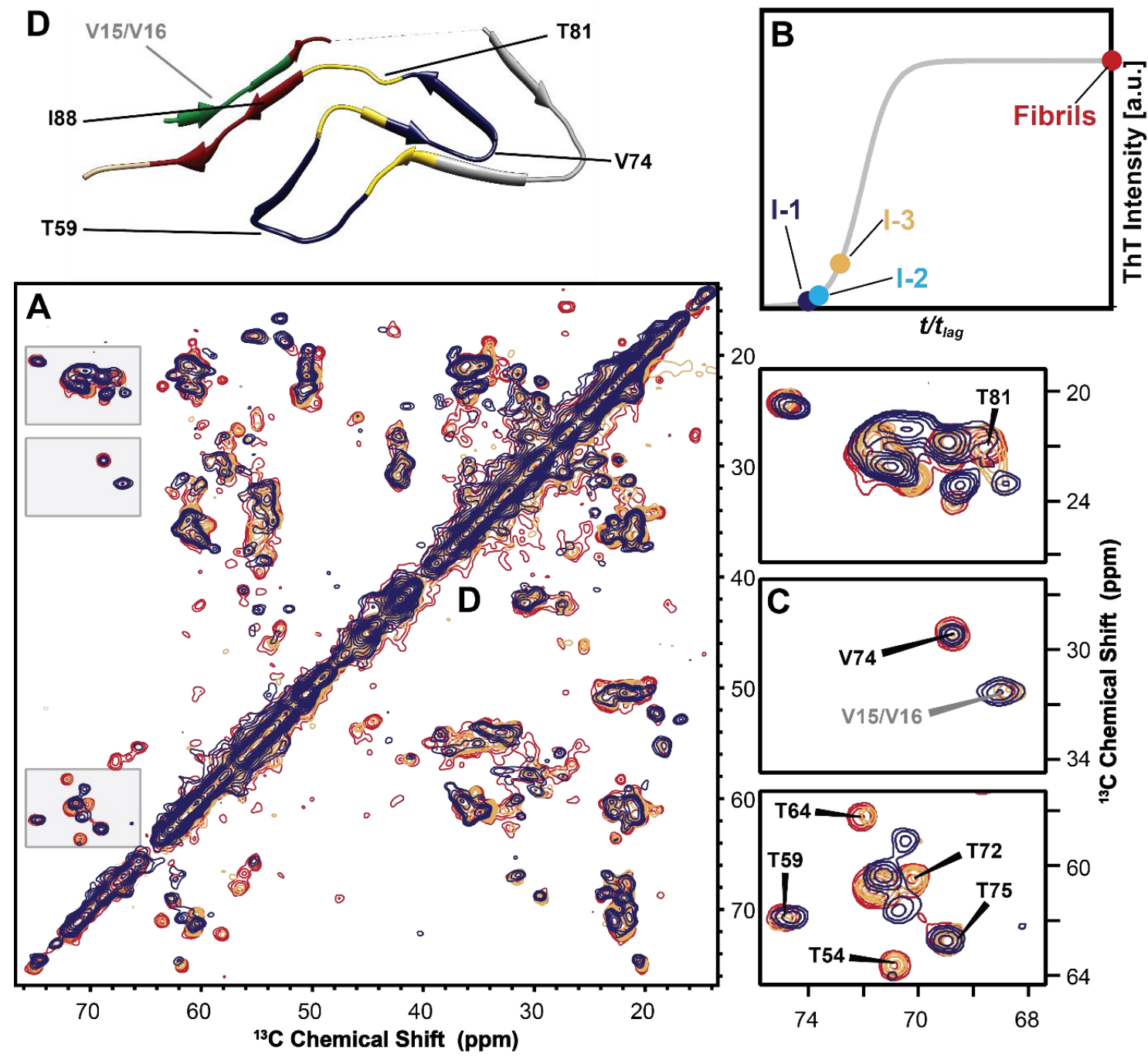

Figure 3.2.7:A) ${ }^{13} \mathrm{C}-{ }^{13} \mathrm{C}$ DARR spectra with $20 \mathrm{~ms}$ of aS intermediates I-1 (dark blue) and I-3 (yellow) prepared in the presence of phospholipids under PMCA conditions to fibrils (red). B) Chronology of the formation of the species based on the ThT values observed at the time of their preparation. C) Cutout regions from $A$ (indicated by gray boxes), comparing key isolated cross-peaks of intermediates with assigned fibril resonances. Most notably, T59, V74 and 75 exhibit the same chemical shift as in fibrils, while the rest of the residues still undergoes chemical shift changes; D) Structured regions observed in 13C-13C DARR spectra of intermediates in fibrils; color coding projected onto PDB entry 6RT0 (polymorph 2) ${ }^{[100]}$. Initial structured regions are centered around the loops at T59, K60 as well as V74 and T75 in intermediate I-1 (dark blue). Intermediate I-3, isolated at a later stage shows fibril-like structure for residues G51-K80 (yellow). Residues T81-F94 obtain their final structure at a later stage, towards full final formation (red). The $\mathrm{N}$-terminal domain of the protein obtains decreasing helical content through the process of aggregation (green). 
Although the full aggregation process was not tracked individually, Figure 3.2.7 A shows the estimated chronology of the observed species. Intermediate 1 (I-1) and Intermediate (I-2) were harvested right before and at $t_{\text {lag, }}$ while Intermediate 3 (I-3) was harvested after significant fibril formation had been detected. Since I-1 and I-2 share an almost identical set of peaks, only I-1 will be discussed in the following. Similarities in I-1 and I-2 ensue from the close temporal proximity on the aggregation time scale.

Despite the lack of sequence assignments, several structural considerations can be made from the spectrum of $\mathrm{I}-1$ and $\mathrm{I}-3$, when comparing them to fibril and monomer spectra. As monomer spectra were not recorded in the course of this work, published data by Comellas et al. as well as Fusco et al. were considered. ${ }^{[91,115]}$ Figure 3.2 .7 shows ${ }^{13} \mathrm{C}-{ }^{13} \mathrm{C}$ DARR spectra of $\mathrm{I}-1$ and I-3 compared to fibril. Several resonances for $\mathrm{I}-1$ can be assigned unambiguously from comparison to fibril (Figure 3.2.8), due to their distinct chemical shifts. These structured residues are T75, V74 and T59, which are featured in the loops between beta sheets in the fibril structure. The observation, that the loops form first, albeit in a different fibril polymorph, agrees with the findings of Comellas et al... ${ }^{[1]}$ Tentatively assigned resonances of neighboring residues (Figure 3.2.8), such as T54, K60 and T64 or A69 and T72, do not yet exhibit the same chemical shifts as in fibrils. Since these resonances exhibit a characteristic $\beta$-sheet chemical shift, conclusion would be, that the sequences V55-T64 and V70-K80 antiparallel $\beta$-sheets with with turns at T59 and V74. This conclusion is supported by the observed antiparallel $\beta$-sheet content, derived from CD-spectroscopy. Several signals of residues connecting these structural elements (N65-V69) are not yet observed, so that the antiparallel $\beta$-sheets, or $\beta$-hairpins are assumed to be independent.

Especially C-terminal residues are not observed in the spectrum of $\mathrm{I}-1$, including S87, 188 and F94, leading to the assumption, that they are still unstructured. Tentantive assignment of T81 suggests, that this residue is already structured, but still undergoes a structural stransition towards the fibril fold. Together with $\mathrm{K} 80$ it forms a turn in the fibril and the observation, that residues V82-F94 are not yet structured, indicates formation of this turn at a later stage in the aggregation process. In conclusion, the intermediate $1-1$ contains two putative $\beta$-hairpins, adopting a structure on pathway towards fibrils, though not sharing key elements of the final fibril fold. 

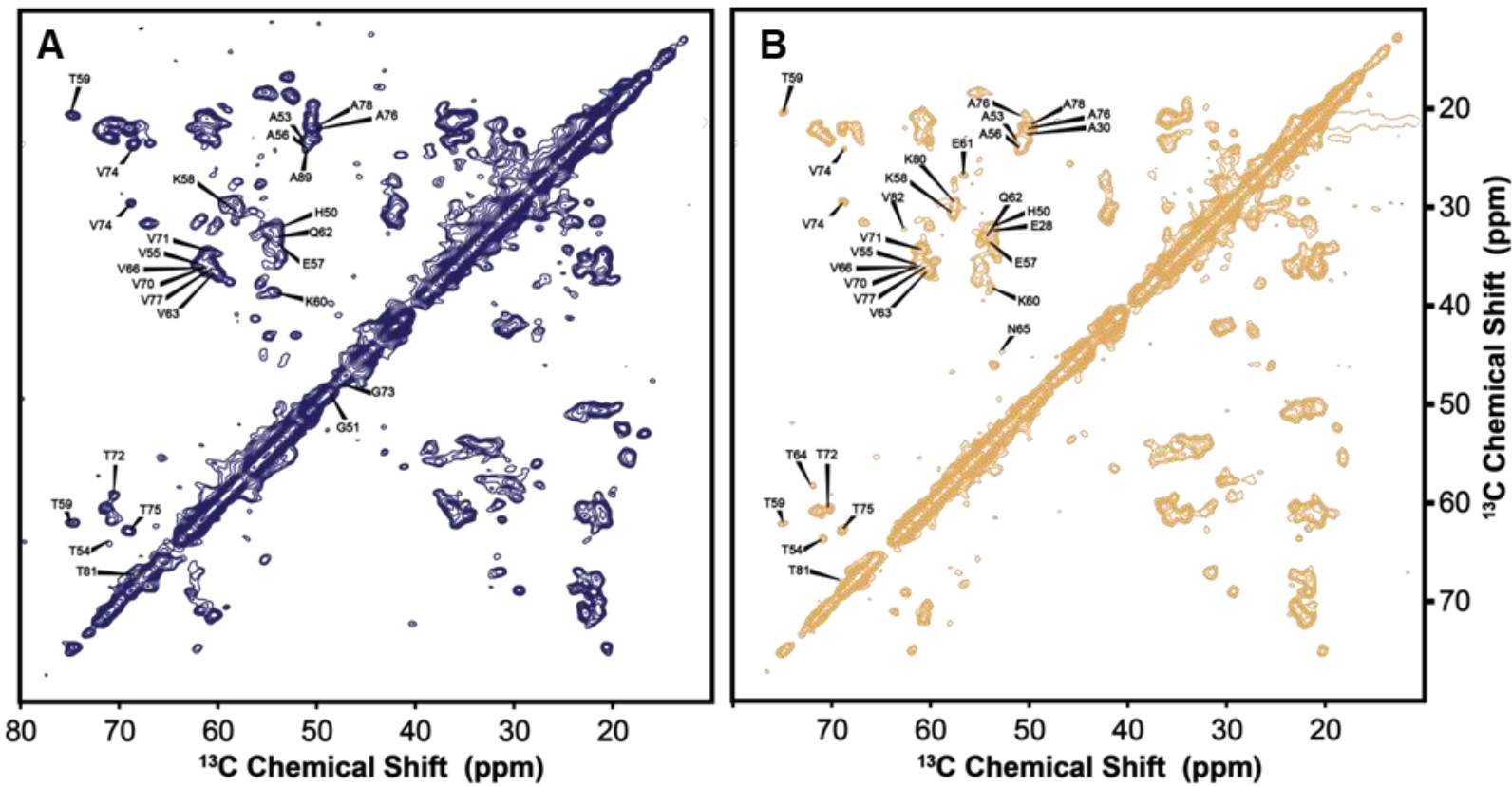

Figure 3.2.8: $2 \mathrm{D}{ }^{13} \mathrm{C}^{13} \mathrm{C}$ Spectra of $\mathrm{A}$ ) $\mathrm{I}-1$ (blue) and $\mathrm{B}$ ) I-3 (yellow) with tentative assignments. Spectra were acquired at $850 \mathrm{MHz}$ with $20 \mathrm{~ms}$ of DARR mixing.

Upon transition of I-1 to $\mathrm{I}-3$ residues $52-82$ adopt chemical shifts very similar to the final structure (Figure 3.2.8). Fibril formation at the point of preparation for I-3 is confirmed by ThT fluorescence and CD, which contrasts with I-3. The conversion of I-1 to I-3 takes approximately 1-2 $\mathrm{h}$ under the conditions at hand. Nevertheless, minor conformational changes still seem to occur on the way to the fibril, as S87, I88 and F94 are observed, but only with minute intensity. Adding to this, A29 is not observed, leading to the conclusion that only the central part of the fibril has yet assembled, whereas both the C-terminal part of the NAC region as well as the $\mathrm{N}$-terminus adopt a disordered structure. Figure 3.2.7 D depicts a tentative chronology of the process of fibril formation. Formation of I-3 is coupled to a pronounced ThT response and aggregation rate, suggesting that the conversion of I-1 to I-3 is a primary nucleation event.

In accordance with the structural insights of Fusco et al on aS oligomers in the presence of phospholipids, the N-terminal domain of Intermediates I- 1 and I-3 adopts an $\alpha$-helical conformation. ${ }^{[00]}$ This finding is derived from a comparison to ${ }^{13} \mathrm{C}-{ }^{13} \mathrm{C}$ DARR spectra of monomers bound to phospholipids, where residues V15 and V16 display a characteristic cross-peak at $67.2 / 31.5$ ppm. A similar peak was found for intermediates by Comellas et al., although without being included in the analysis. ${ }^{[91]}$ Alongside this signal, three distinct crosspeaks in the range of $55-52 \mathrm{ppm}$ and $19-17 \mathrm{ppm}$ hint towards Alanine residues in a helical conformation. It is therefore reasonable to assume an $\alpha$-helical conformation for the sequence ${ }_{11} A K E G V V A A A_{19}$. in the intermediate species. Membrane binding of oligomers via this helical N-terminal domain was previously reported by Fusco et. al.. ${ }^{[90]}$ The presence of monomers is excluded, as these cross-peaks are still featured in fibril spectra, albeit with 
minor intensities. Moreover, solution NMR showed, that conversion of membrane bound monomers to aggregates is fast, resulting in low levels of membrane bound monomer.

The observation of an N-terminal domain is similarly made for fibrils. This is in accordance with the strong tendency of fibrils to bind to SUVs and the finding, that lipids can cover whole fibrils (Figure 3.1.6). Anchoring of aS to phospholipid membranes by the $\mathrm{N}$-terminal domain hence persists through the whole aggregation process.
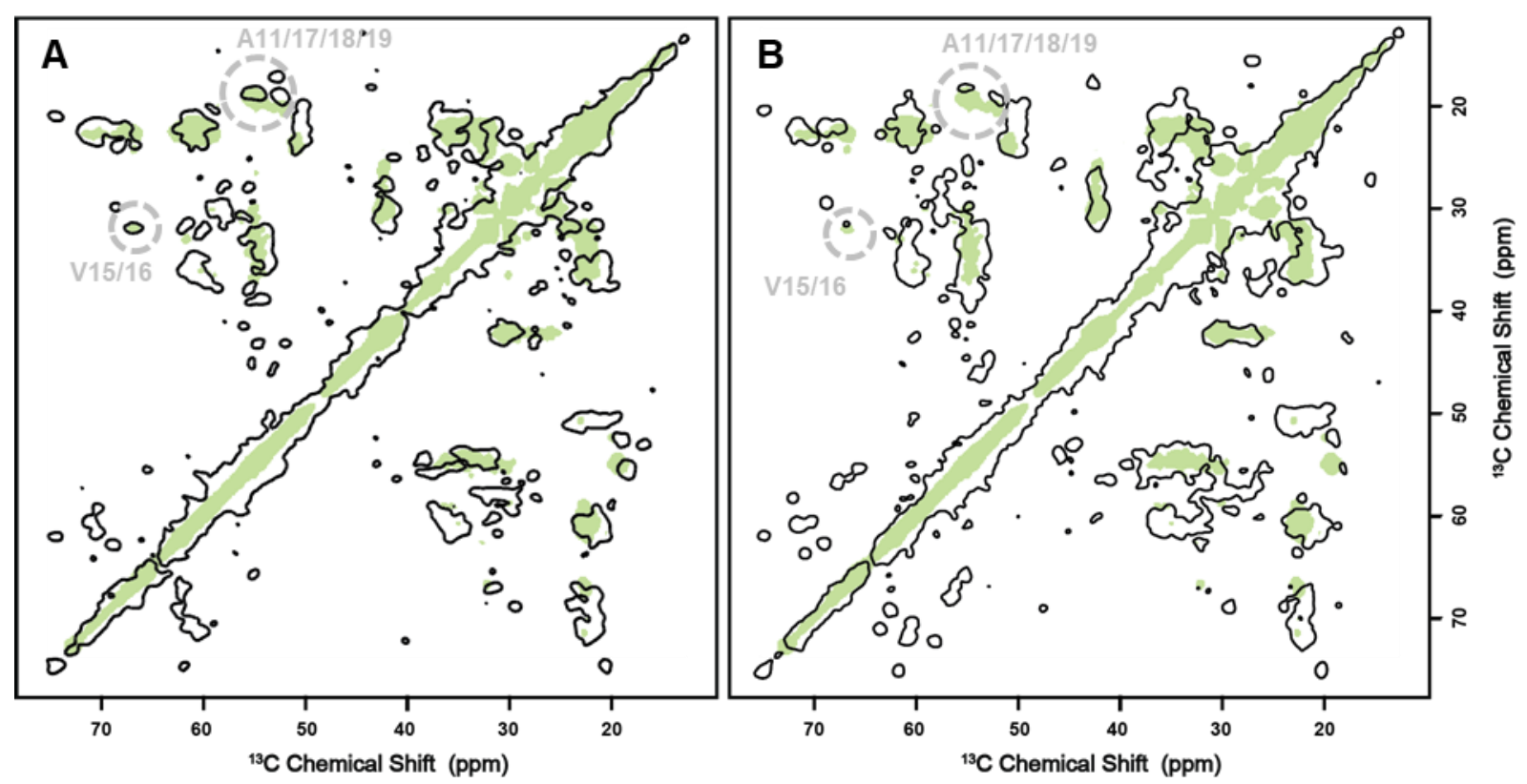

Figure 3.2.9: Comparison of $\alpha \mathrm{S}$ oligomers $(\mathrm{A})$ and fibrils $(\mathrm{B})$ in this work (black outline) with monomer spectra in the presence of phospholipids (green) published by Comellas et al. JACS 2012. A: Both fibrils and oligomers display chemical shifts, characteristic for a helical conformation in residues V15/V16. 


\subsection{Interaction of anle138b with $\alpha S$ aggregates}

\subsubsection{Influence of anle138b on $\alpha$-synuclein aggregation kinetics}

One of the major goals of this thesis is the identification of a mechanistic influence of anle138b on disease relevant protein species. In order to probe its influence on the aggregation of $\alpha S$, PMCA studies were carried out using the same methods as described above.

A

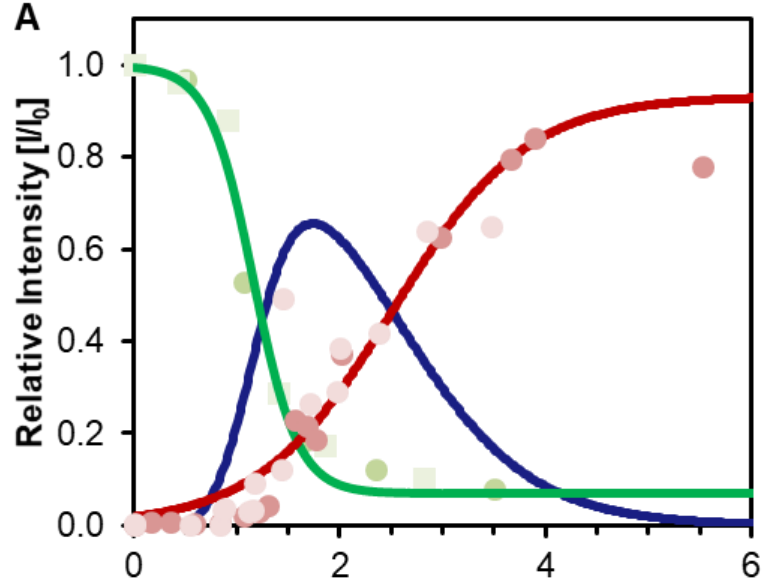

B

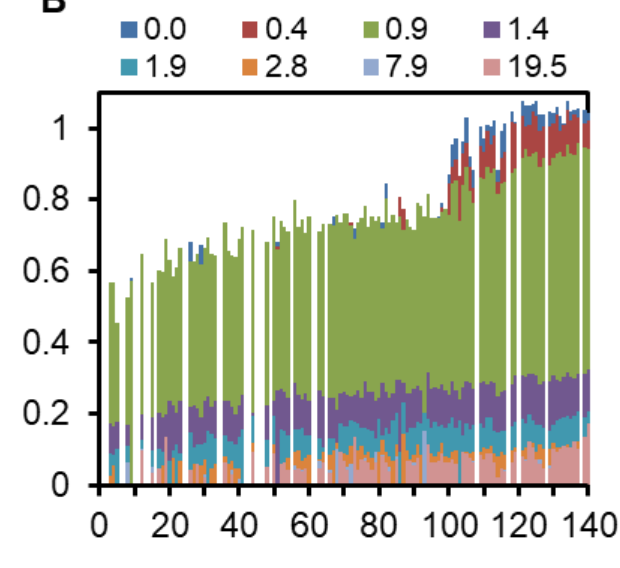

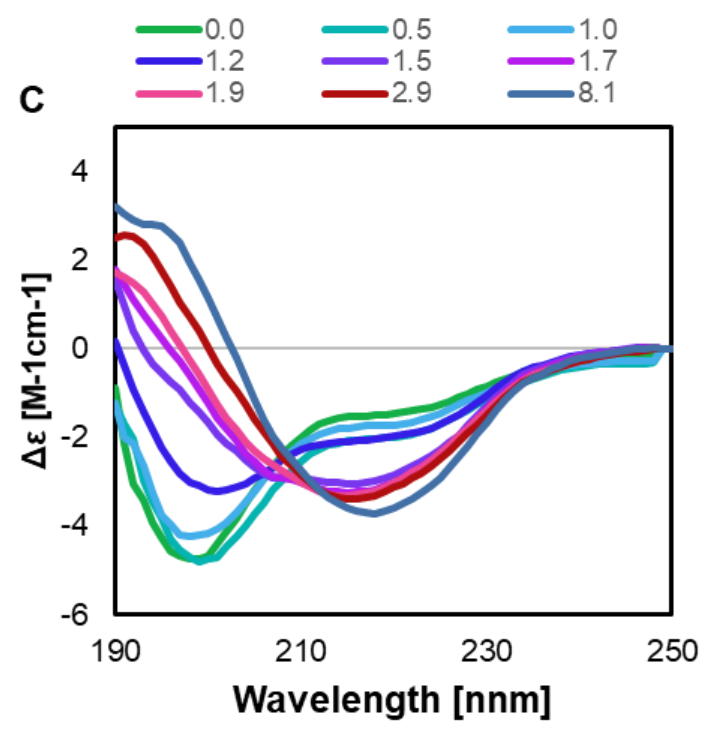

Helix Antiparallel Parallel Turn Others

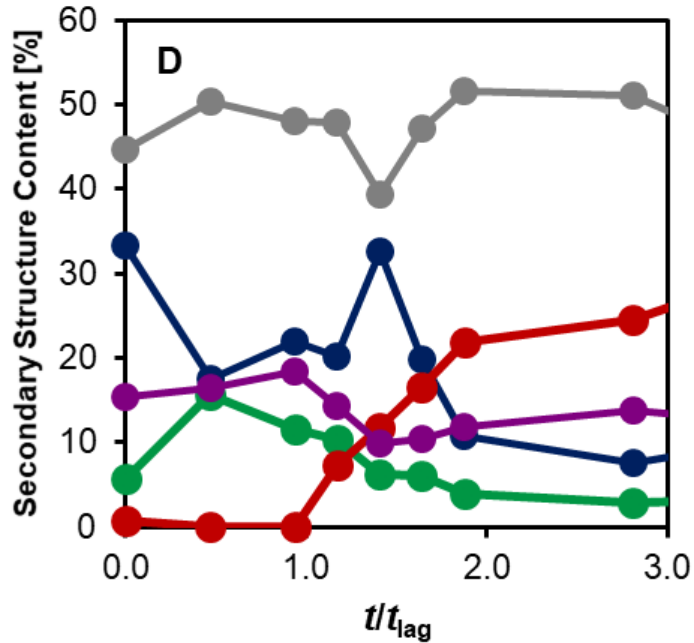

Figure 3.3.1: Kinetics of $\alpha S$ in the presence of anle138b containing vesicles: A) Oligomer concentration (blue) as estimated from ThT fluorescence (red) and NMR intensity (green); B) Development of Intensities in ${ }^{1} \mathrm{H}-{ }^{15} \mathrm{~N}-\mathrm{HSQC}$; C) CD-Spectra; D) secondary structure content derived by BeStSel

The differences in lag times determined from ThT fluorescence observed for anle138b compared to control samples are in accordance with the usual fluctuations. A kinetic influence on fibril formation under the conditions used here can therefore be excluded. As 
illustrated by the disappearance of the attenuation profile characteristic to membrane binding (Figure 3.3.1 B), aggregation proceeds in a membrane bound state. This is accompanied by a decrease in lipid signal intensity in NMR spectra. In the presence of anle138b the fraction of oligomers is estimated to make up more than $60 \%$ of the protein species, much higher than in its absence. At equilibrium conditions after fibril formation, maximum ThT fluorescence intensities reach values comparable to those in the absence of compound. This finding is independent of the agitation method, as an inhibition or reduction of fibril formation was observer neither under shaking, nor under quiescent conditions.

Secondary structure calculation from CD during aggregation in the presence of anle138b reveals no increase in anti-parallel $\beta$-sheet before fibril formation. If this is correct, it would mean that anle138b inhibits anti-parallel $\beta$-sheet and drives formation of parallel $\beta$-sheet directly. Such a mechanism is however difficult to imagine, given the results by ssNMR on intermediates, as will be discussed later on. Nevertheless, all of the secondary structure contents should be analyzed with care, given the experimental limitations mentioned above. It is not clear at this point, how much influence the experimental limitations influence the secondary structure calculation. The application of FITR or synchrotron radiation CD might overcome these limitations and help in the confirmation of anti-parallel $\beta$-sheet containing intermediates. ${ }^{[153,160]}$

The absence of an inhibitory function of anle138b on aS fibril formation contradicts the reduced load of deposits found in PD mouse models. ${ }^{[131]}$ Taking into account the complexity of the physiological environment, in vitro assays might be lacking a transporter for anle138b, resulting in failure to bind to aggregates in a quantitative manner required for inhibition. ${ }^{[167]} \mathrm{A}$ readily available transporter protein is Serum Albumin, which has been shown to be involved in the transport of hydrophobic drug molecules, such as ibuprofen and aspirin. ${ }^{[168,169]}$ Furthermore, it is involved in the transport of fatty acids and their regulation in cellular environments. ${ }^{[170]}$ Here, BSA was used as an affordable model protein. BSA has multiple binding sites for hydrophobic molecules and can reportedly mobilize drug molecules from a micellar environment. ${ }^{[171]}$

A complex of anle138b and BSA was prepared by addition of a solution of the compound in DMSO to the protein in buffer. Upon binding to BSA the compound UV spectrum experiences a small blue shift from $270 \mathrm{~nm}$ to $263 \mathrm{~nm}$, when compared to DMSO, suggesting a slightly more hydrophobic environment (Figure 3.3.2 A). At saturation levels, the ratio of anle138b to BSA was estimated to be $3: 2$, which is comparable to the ratios observed for other hydrophobic molecules and indicates that more than one binding site in BSA is occupied. ${ }^{[169]}$ 

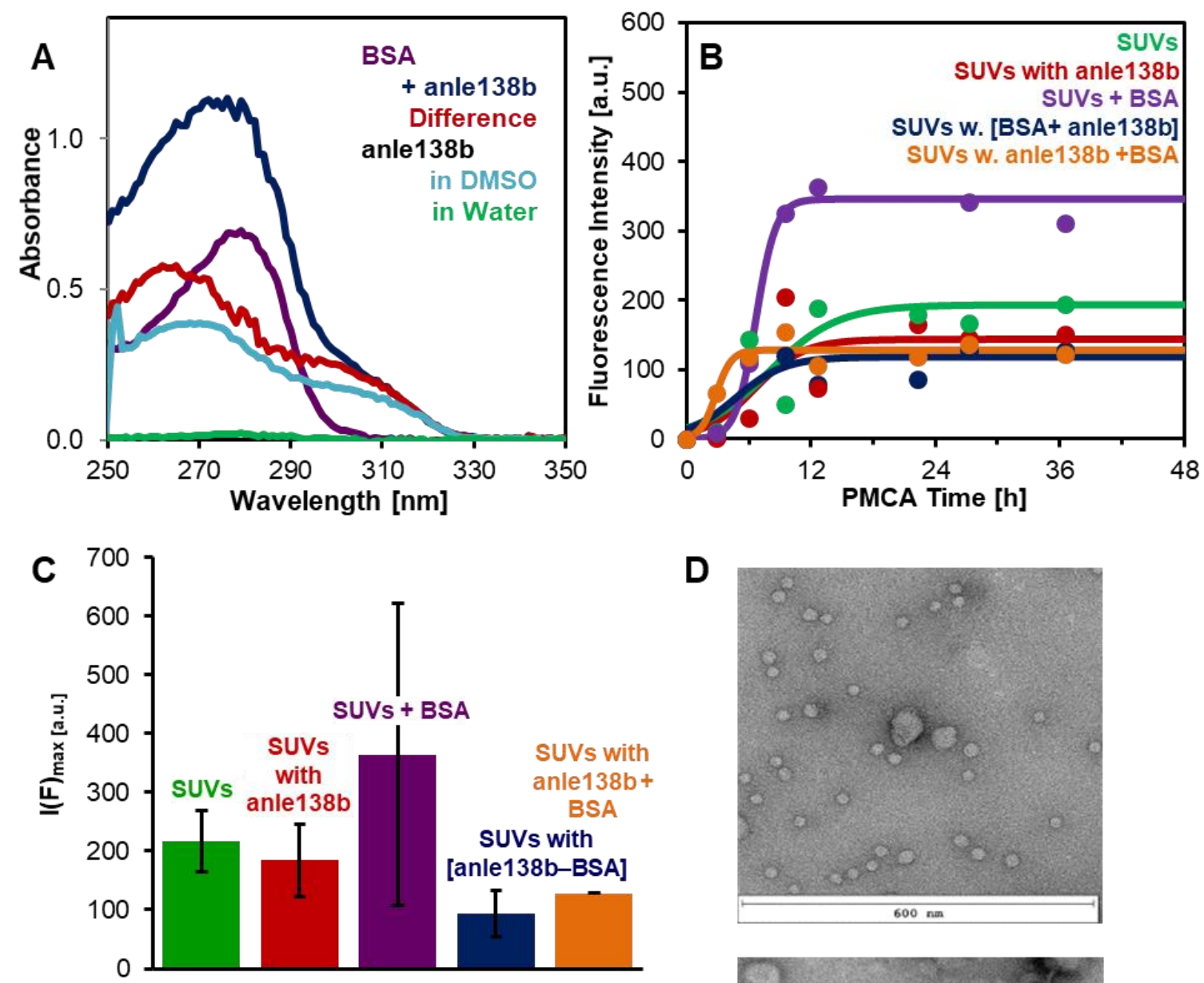

E
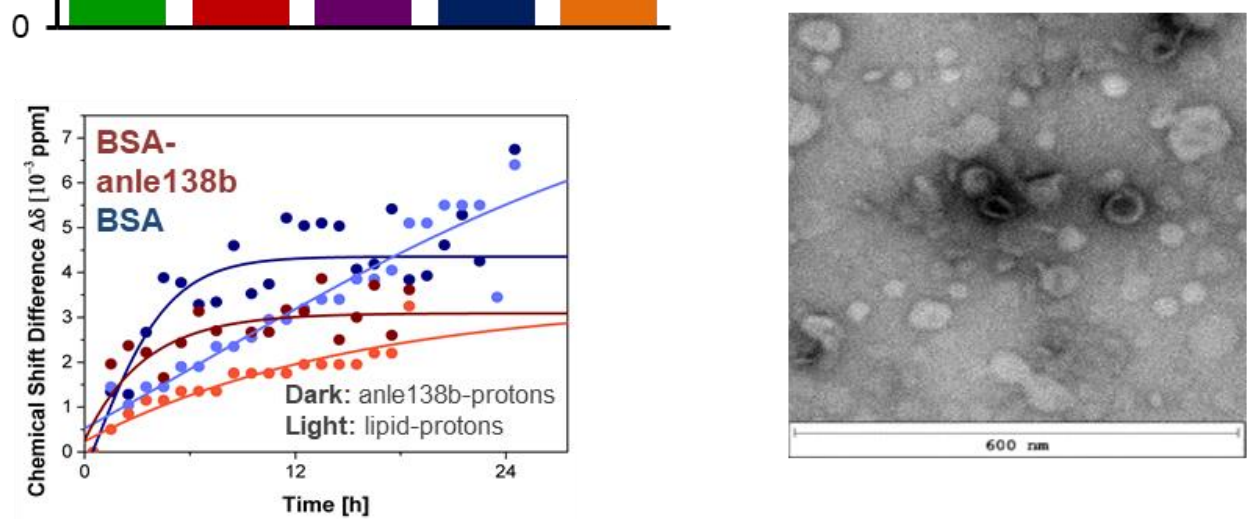

Figure 3.3.2: Experiments on the influence of anle138b on $\alpha S$ aggregation using $B S A$ as a mediator. A: UV spectra confirming complexation of anle $138 \mathrm{~b}$ by BSA; B: ThT assays on the effect of [anle138b-BSA] complex of aS aggregation in comparison to controls; C: Quantification of aS aggregation under the influence of anle138b and BSA compared to controls, maximum ThT values were calculated from fitting a sigmoidal function to kinetic data; D: Electron micrographs of SUVs of POPA and POPC before (left) and after (right) incubation with BSA over night; E: Influence of BSA on SUVs tracked by ${ }^{1} \mathrm{H}$ 1D NMR, in the presence (red) and absence (blue) of anle138b. Resonances of both lipid (light) and anle138b (dark) experience chemical shift changes due to increase in vesicle diameter by vesicle fusion.

Using these conditions, the achievable compound concentrations were similar to when phospholipids are used as a carrier. The complex shows remarkable stability, resisting dialysis against a 1000-fold volume for over $24 \mathrm{~h}$ with minor losses in compound. Indeed, the use of the anle138b-BSA complex in PMCA aggregation experiments resulted in a decrease 
in the maximum ThT fluorescence (Figure 3.3.2 B). A significant reduction in the lag time of aS aggregation was not observed. Surprisingly, the addition of compound-free BSA resulted in an increase in the maximum ThT fluorescence. An enhancement of aS aggregation in the presence of BSA has previously been attributed to molecular crowding, however the study used concentrations almost 20 times higher $(900 \mu \mathrm{M})$ than in this work $(50 \mu \mathrm{M}) .{ }^{[172]}$ When investigating phospholipid vesicles in the presence of BSA, Dynamic Light Scattering (DLS) revealed an increase of the vesicle diameter from $40 \mathrm{~nm}$ to $70 \mathrm{~nm}$ within $24 \mathrm{~h}$. Investigation of the same samples by EM corroborated this finding (Figure 3.3.2 D). Under similar conditions Schenkman et al. reported fusion of SUVs induced by BSA, resulting in an increase of the diameter PC-vesicles from $50 \mathrm{~nm}$ to $100 \mathrm{~nm}$ within 14 h. ${ }^{[173]}$ In another study BSA was shown to adsorb on negatively charged lipids, resulting in disruption of the bilayer and dye leakage for concentrations relevant to this work. ${ }^{[174]}$ Such a behavior is not reproduced in the absence of BSA, as vesicles are stable for days if not perturbed. Although it was not tested, whether vesicle fusion takes place under PMCA conditions, the disruption of membranes might very well cause stronger aggregation due to the exposure of hydrophobic surfaces. This might add an additional stress during the resting phases in the PMCA assay, causing amplification of the total aggregate.

The fusion of vesicles is reduced in the presence of the anle138b-BSA complex, probably due to partial blocking of the relevant binding site for BSA-lipid binding (Figure 3.3.2 E). In $1 \mathrm{D}{ }^{1} \mathrm{H}-\mathrm{NMR}$ spectra the fusion causes a broadening of the resonances from anle138b as well as from the phospholipids, which tumble slower due to the increased size of the vesicles. Judging from the chemical shift change vesicle fusion is reduced by about $25 \%$, but further studies are needed to obtain an actual quantification.

Considering, that BSA alone causes an increase in aggregation, it is even more remarkable that a complex of BSA and anle138b reduces aS aggregation. It seems that on top of a potential inhibitory function on fibril formation, anle138b has a membrane protective function, although studies under milder aggregation conditions are needed to support both statements. 


\subsubsection{Influence of anle138b on aS fibril formation}

The absence of a kinetic inhibitory mechanism of anle138b of aS aggregation makes it all the more likely that its efficacy in various disease related models might be best explained by an interference of structural nature. Structurally related compounds had been shown to bind to fibrillar aS by means of crosslinking and computational docking models. ${ }^{[175]}$

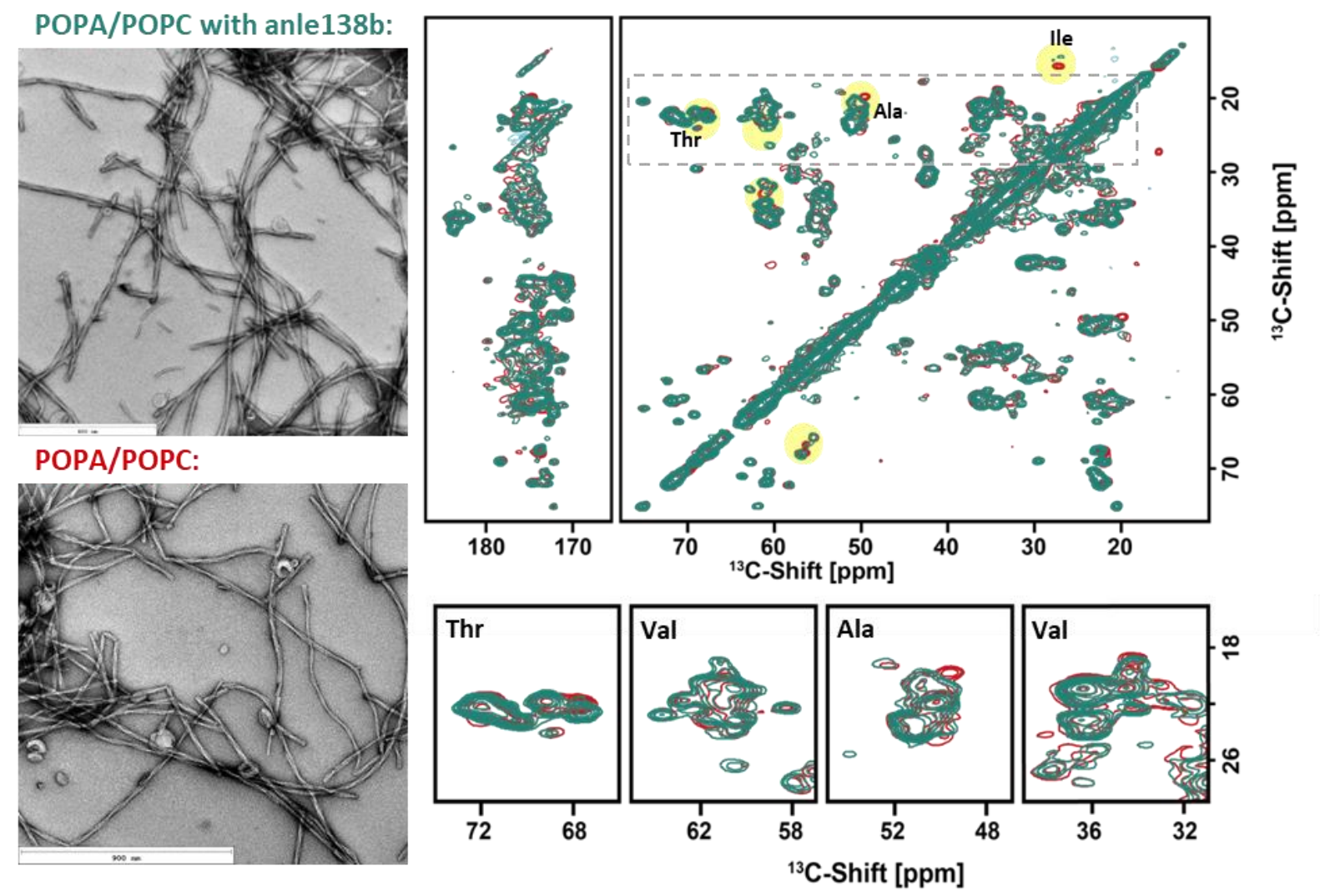

Figure 3.3.3: aS fibrils grown in the of SUVs of POPA/POPC under PMCA conditions; Left: TEM micrographs of fibrils grown in the presence (top) and absence (bottom); Right: ${ }^{13} \mathrm{C}-{ }^{13} \mathrm{C}$ DARR spectra of fibrils grown in the presence (green) and absence (red) of anle138b.

Fibrils of $(\mathrm{U}){ }^{13} \mathrm{C},{ }^{15} \mathrm{~N}$ aS were grown in the presence of anle138b containing vesicles of POPC and POPA and compared to fibrils grown in the absence of compound. Morphological analysis by TEM does not reveal major differences in either helical twist, width or abundance of observed structures. Images in the presence of anle138b more frequently featured single filaments, yet statistical relevance is to be doubted.

Accordingly, ${ }^{13} \mathrm{C}-{ }^{13} \mathrm{C}$ DARR spectra revealed minor chemical shift changes caused by the compound, while the structural core of the fibrils appears unaltered (Figure 3.3.3). Analysis of these changes is complicated, due to the crowded appearance on the spectra, however isolated cross/peaks that are influenced, were assigned to T81, S87 and 188. These peaks experience a chemical shift change, along with a significantly decreased signal intensity, 
indicating a more dynamic or disordered structure when bound to anle138b. Other crosspeaks could not be assigned unambiguously, however the residue types include Alanine and Valine.

The perturbances in structure apparently affect the region of the fibril structure, for which significant differences between the polymorph prepared by PMCA and polymorph 2 are observed (see chapter 3.2.1). Since alignment and orientation of the residues in this region is not known, exact structural influences of anle138b are difficult to predict. Assuming that arrangement of the chain in the fibril cross section is not substantially different, S87 and 188 are located at the edge of the structured part at G93/F94/V95. Residue T81 is located in a turn right before this C-terminal part of the structured core. However, in polymorph 2 the aforementioned sequence is capped by part of the $\mathrm{N}$-terminal chain, decreasing its accessibility. It has to be clarified, whether a similar alignment of the chain is present in the polymorph prepared under PMCA in the presence of lipids. A potential interaction of anle138b might be the binding to residues in the sequence ${ }_{81}$ TVEGAGSI ${ }_{88}$ during fibril formation, which induces the observed perturbances. Upon final formation of the structured core however, the compound will most likely bind to more disordered regions, due to the increased accessibility of the protein backbone, as suggested by MD-simulations (see below). ${ }^{[139]}$ 


\subsubsection{DNP-enhanced solid-state NMR experiments}

Oligomers of $\alpha S$ are reportedly transient in nature and rapidly interconvert to eventually form fibrils, a finding clearly corroborated by our own experimental observations. One of the options to slow down such rapid processes is freezing of samples, arresting intermediate species in their current state. DNP-enhanced ssNMR operates at temperatures of around $100 \mathrm{~K}$ and therefore allows the measurement of spectra of such frozen samples. ${ }^{[62]}$ This technique utilizes the microwave driven transfer of polarization from the electron spins of a biradical to the ${ }^{1} \mathrm{H}$ nuclei of the molecule of interest, leading to an enhancement in sensitivity. ${ }^{[176]}$

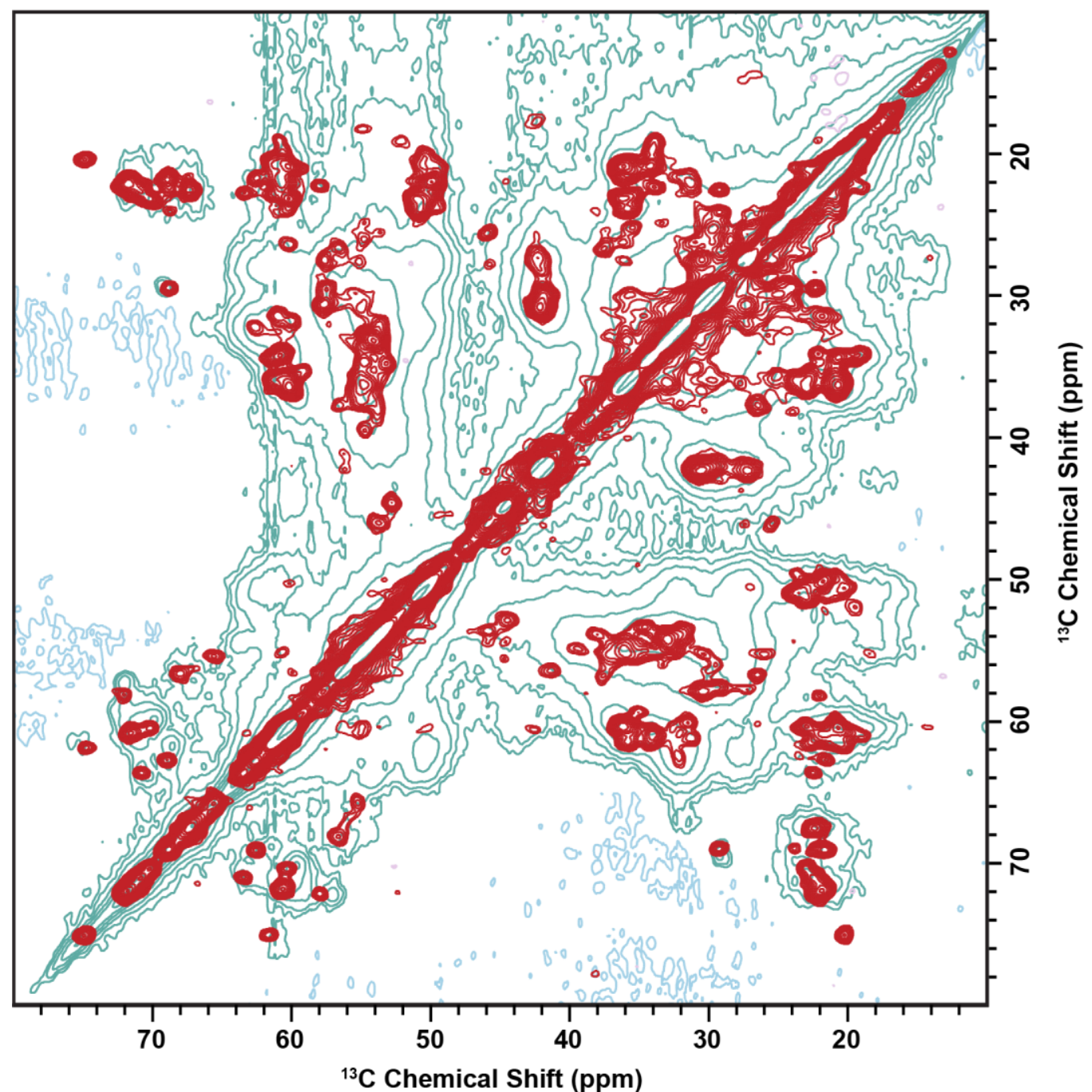

Figure 3.3.4: Comparison of $13 \mathrm{C} 13 \mathrm{C}$ RFDR spectrum of fibrils acquired at $100 \mathrm{~K}$ (green) with ${ }^{13} \mathrm{C}-{ }^{13} \mathrm{C}$ DARR at RT.

To study oligomers and fibrils under these conditions, the biradical TEMTRIPOL was used for enhancement. Aggregate samples were prepared identically to other ssNMR samples until the point of rotor packing. The mixture of lipids, anle138b and aggregates was treated 
with a solution of ${ }^{13} \mathrm{C}$-depleted $d_{8}$-glycerol, to avoid water crystallization during freezing. In addition to this, $d_{64}$-POPC and $d_{64}$-POPA were used, to improve sensitivity of the membrane associated molecules. The achieved enhancement factor on these samples was in the order of $\sim 6-7$.

A comparison of DNP-enhanced ${ }^{13} \mathrm{C}-{ }^{13} \mathrm{C}$ correlation spectra with radiofrequency driven dipolar recoupling (RFDR) of aS fibrils measured at $100 \mathrm{~K}$ with ${ }^{13} \mathrm{C}-{ }^{13} \mathrm{C}$ DARR spectra of similar samples at RT showcases the drastic difference in linewidth (Figure 3.3.4). At $100 \mathrm{~K}$ most of the conformations of the molecules under investigation are frozen out. This includes residues that are located in more dynamic regions, leading to heterogeneity. ${ }^{[62]}$ As a result, the small structural differences between oligomeric and fibrillary aggregates are indistinguishable. Nevertheless, it is possible to identify several characteristic peaks in the low temperature spectrum, with only minor chemical shift differences from data at $300 \mathrm{~K}$.

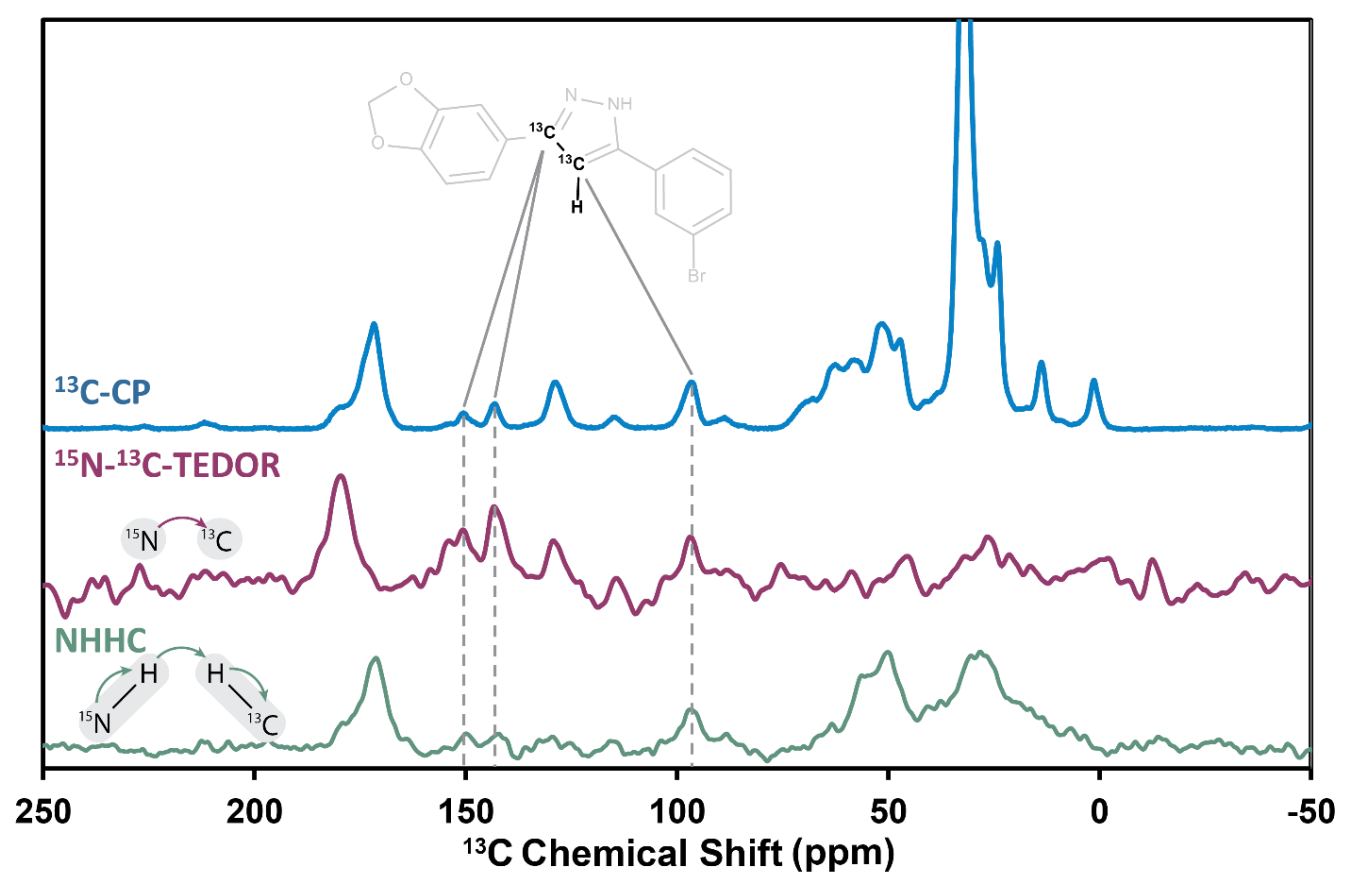

Figure 3.3.5: DNP-enhanced ssNMR on ${ }^{15} \mathrm{~N}-\alpha \mathrm{S}$ aggregates in the presence of $3,4-{ }^{13} \mathrm{C}$-anle $138 \mathrm{~b}$ containing phospholipids; Top: ${ }^{1} \mathrm{H}-{ }^{13} \mathrm{C}-\mathrm{CP}$, Center: ${ }^{15} \mathrm{~N}-{ }^{13} \mathrm{C}-\mathrm{TEDOR}$, Bottom: hNHHC.

In order to probe the interaction of the small molecule anle138b with the respective aggregates, several labelling schemes were employed. Samples of $U-15 N-\alpha S$ in the presence of $3,4-{ }^{13} \mathrm{C}$-anle $138 \mathrm{~b}$ allowed the measurement of ${ }^{15} \mathrm{~N}-{ }^{13} \mathrm{C}$-TEDOR, as well as hNHHC spectra (Figure 3.3.5). Both of these experiments allow transfer of magnetization via dipolar coupling of between the ${ }^{15} \mathrm{~N}$ and ${ }^{13} \mathrm{C}$ nuclei or their respective attached protons. Assignments of compound signals is readily achieved in ${ }^{13} \mathrm{C}-{ }^{13} \mathrm{C}$ RFDR spectra (Figure 3.3.6 A). The observability of anle138b resonances in DNP spectra is in contrast to experiments without enhancement at RT. This hints to anle138b being dynamic in the bilayer 
as well as in its protein bound states. A set of two signals is obtained for the C-3 of the compound, as the exchange between the tautomers of the pyrazole ring is very slow at $100 \mathrm{~K}$. In a $1 \mathrm{D}{ }^{15} \mathrm{~N}-{ }^{13} \mathrm{C}$-TEDOR with $25 \mathrm{~ms}$ of mixing time, transfer from ${ }^{15} \mathrm{~N}$ of the protein, to both labelled ${ }^{13} \mathrm{C}$ of anle138b is observed.

A

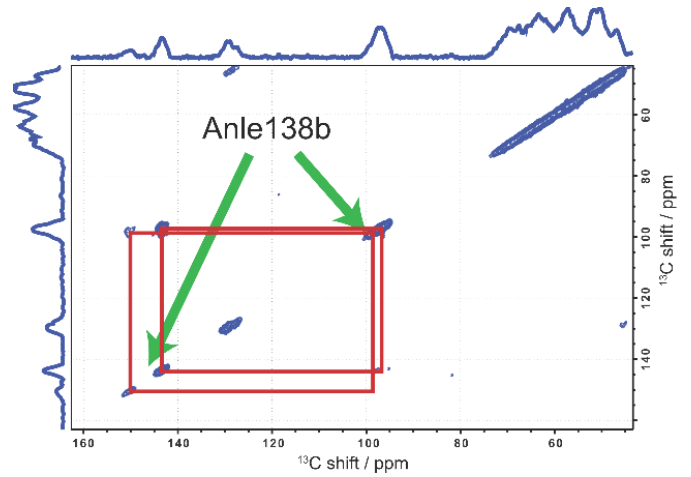

B

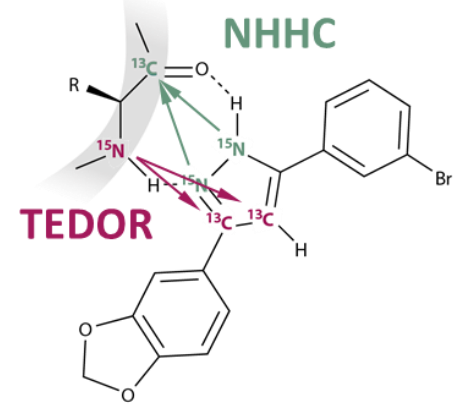

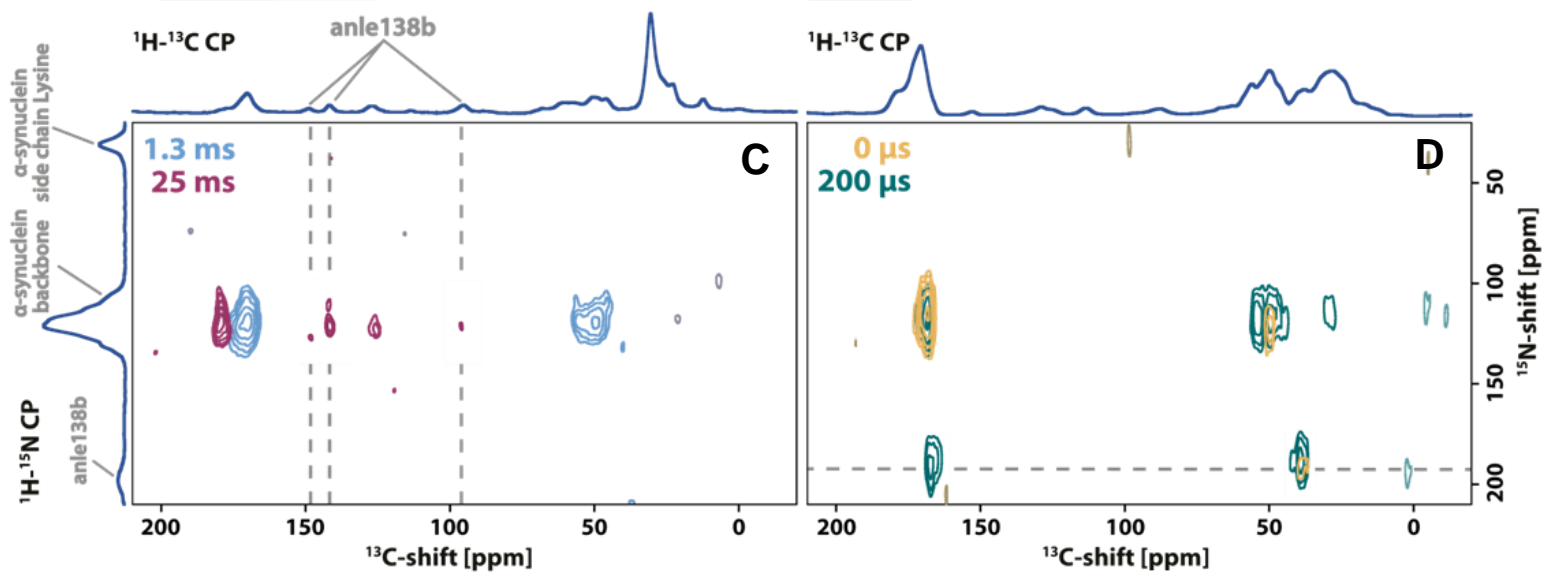

Figure 3.3.6: A) RFDR Spectrum of anle138b in lipids with $2.56 \mathrm{~ms}$ mixing time; B) tentative binding mode of anle to backbone showing the transfer pathways for ${ }^{15} \mathrm{~N}-{ }^{13} \mathrm{C}$-TEDOR (magenta) and hNHHC (green); C) TEDOR on 15N oligomers with 13C anle D) NHHC on 13C fibrils with $15 \mathrm{~N}$ anle138b. Mixing times for TEDOR and hNHHC spectra are displayed in the top left.

This observation means that the distance of the compound is likely in a range of $5 \AA$. This is consistent with the signal at $180 \mathrm{ppm}$, which most likely originates from protein side-chain C' nuclei. A similar result is obtained, when recording a 1D hNHHC spectrum with $200 \mu \mathrm{s}$ mixing time on the same sample.

The observation made by MD-simulations, that anle138b binds to the backbone of misfolded proteins, is confirmed by $2 \mathrm{D}-{ }^{15} \mathrm{~N}-{ }^{13} \mathrm{C}-\mathrm{TEDOR}$ spectra (Figure 3.3.6), by transfer of magnetization from protein ${ }^{15} \mathrm{~N}$ around $120 \mathrm{ppm} \cdot{ }^{[139]}$ Even though individual protein resonances are not resolved, transfer from Lysine $N_{\varepsilon}$, expected at $37 \mathrm{ppm}$, can be excluded. Figure 3.3.6 shows a schematic representation of a potential binding mode of the compound to the backbone. Distances in this tentative conformation are approximately $4.5 \AA$ and $5 \AA$ 
between the ${ }^{15} \mathrm{~N}$ of the amide and C-3 and C-4 of anle138b respectively and therefore well in range of the expected values for a TEDOR experiment.

To further elucidate the nature of this interaction isotope labelling was reversed, yielding aggregates of $\mathrm{U}-{ }^{13} \mathrm{C}-\alpha \mathrm{S}$ in the presence of $1,2-{ }^{15} \mathrm{~N}$-anle138b. This sample composition allowed the measurement of $2 \mathrm{D}$ hNHHC experiments, probing the correlation of the ${ }^{1} \mathrm{H}$ bearing nitrogen of the pyrazole, exhibiting a distinct chemical shift at $193 \mathrm{ppm}$, with ${ }^{13} \mathrm{C}$ of the protein. At a mixing time of $200 \mu \mathrm{s}$, transfer from compound to the C'-resonances of the protein is observed. Further cross-peaks are listed in Figure 3.3.8, displaying chemical shifts typical for $\mathrm{C}_{\alpha^{-}}$and $\mathrm{C}_{\beta}$-resonances.

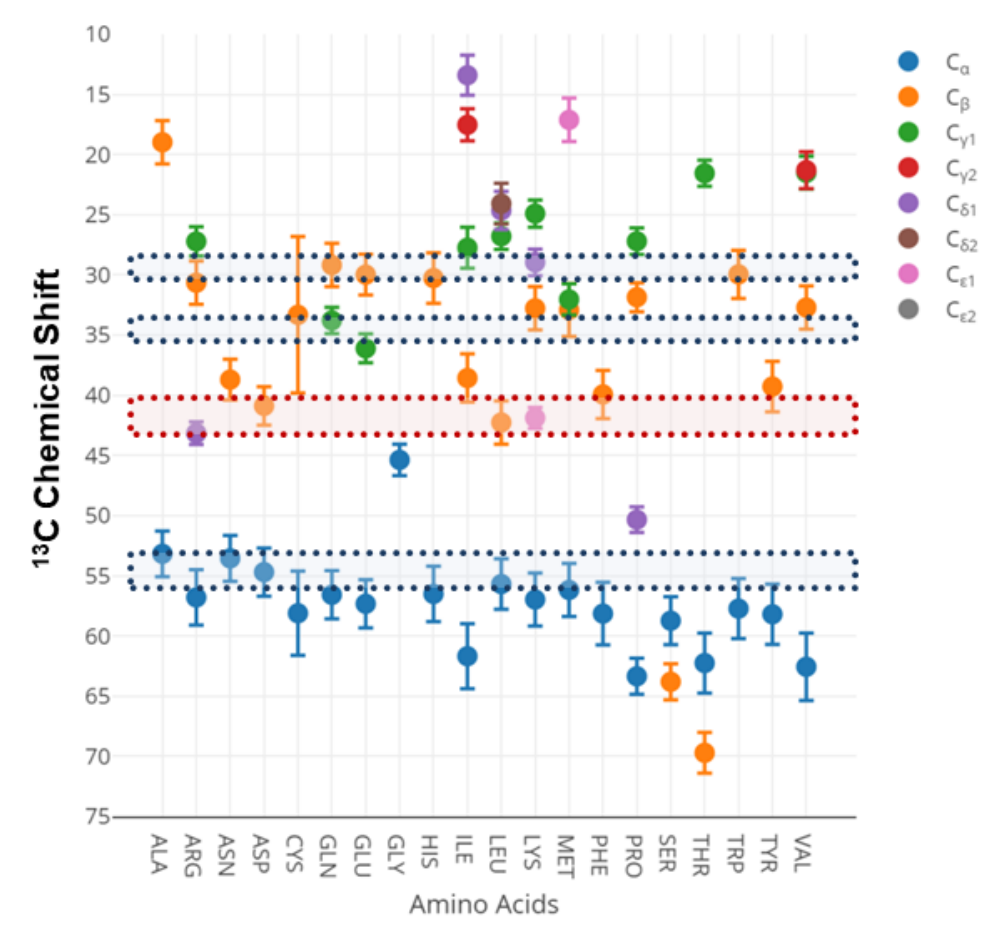

Figure 3.3.7: Characteristic chemical shifts of aliphatic carbons for all 20 amino acids. Reproduced from (http://bionmrcore.umich.edu/index.php/category/statistics/); Highlighted areas represent the observed correlations from anl138b to oligomer (blue) and fibrils (red) in $\mathrm{NHHC}$ spectra.

When the mixing delay in the $\mathrm{hNHHC}$ experiment is omitted ( $\left.\mathrm{T}_{\mathrm{mix}}=0 \mu \mathrm{s}\right)$, a cross-peak is observed at $42.4 \mathrm{ppm}$, as for longer mixing times. Without proton mixing, ${ }^{1} \mathrm{H}$ to ${ }^{13} \mathrm{C}$ magnetization transfer is achieved during cross polarization, meaning that correlations of only the most proximate nuclei will be detected. Comparison with statistical chemical shift values (Figure 3.3.7), shows a variety of potential amino acid residue, that might be involved in the interaction with anle138b. A study on fibrils formed by the short peptide GNNQQNY utilizing DNP-enhanced solid-state NMR showed that chemical shifts recorded at $100 \mathrm{~K}$ can display deviations of $\Delta \delta\left({ }^{13} \mathrm{C}\right)= \pm 1 \mathrm{ppm}$, when compared to room temperature. ${ }^{[62]}$ Considering these differences, Asp, Asn, Leu, Ile, Phe and Tyr are identified by their 
characteristic $\mathrm{C}_{\beta}$-shift, as well as Lys $\left(\mathrm{C}_{\bar{\delta}}\right)$ and $\mathrm{Gly}\left(\mathrm{C}_{\alpha}\right)$. This broad array of potential binding leaves considerable ambiguity, however in combination with complementary techniques a conclusion regarding a binding site is facilitated, as will be discussed later on.

When hNHHC experiments were performed on oligomers of $\mathrm{U}-{ }^{13} \mathrm{C}-\alpha \mathrm{S}$ in the presence of $1,2-{ }^{15} \mathrm{~N}$-anle138b, the set of cross-peaks obtained at $200 \mu \mathrm{s}$ of mixing time differs from the one for fibrils (Figure 3.3.8 A). All compound to protein correlations from hNHHC spectra are listed in (Figure 3.3.8 C). The resonances in the presence of oligomers are characteristic for Lys, GIn and Glu, which are all highly abundant in the aS sequence. Inspection of the ${ }^{13} \mathrm{C}$ ${ }^{13} \mathrm{C}$ - DARR spectrum of $\mathrm{I}-1$, which was prepared at similar $t / t_{\text {lag }}$ values, shows that the region of interest $\left(\delta_{\mathrm{obs}}= \pm 1 \mathrm{ppm}\right)$ does not bear any cross-peak. In contrast to this fibril spectra do carry a set of peaks in this area, which is observed for neither of the intermediate spectra. Anle138b hence seems to bind to a region in aS oligomers, which is dynamic and disordered and only forms much later in the aggregation process. This is in line with the fact, that observation by ${ }^{13} \mathrm{C}-{ }^{13} \mathrm{C}$-RFDR experiments at RT was not possible. The cross-peaks for this potential interaction site belong to the residues that have not yet been assigned. Gath et al. found similar peaks and assigned them to E20, K43, K58. K96 and K97. The first and the last two residues belong to residues that were not yet assigned in the fibrils studied here, whereas K58 obtains a distinctly different chemical shift and is part of a loop, that is assumed to adopt a different conformation than polymorph 2. Completion of the sequence assignments of $\alpha \mathrm{S}$ fibrils will reveal, whether these residues are involved in the interaction with anle138b. 


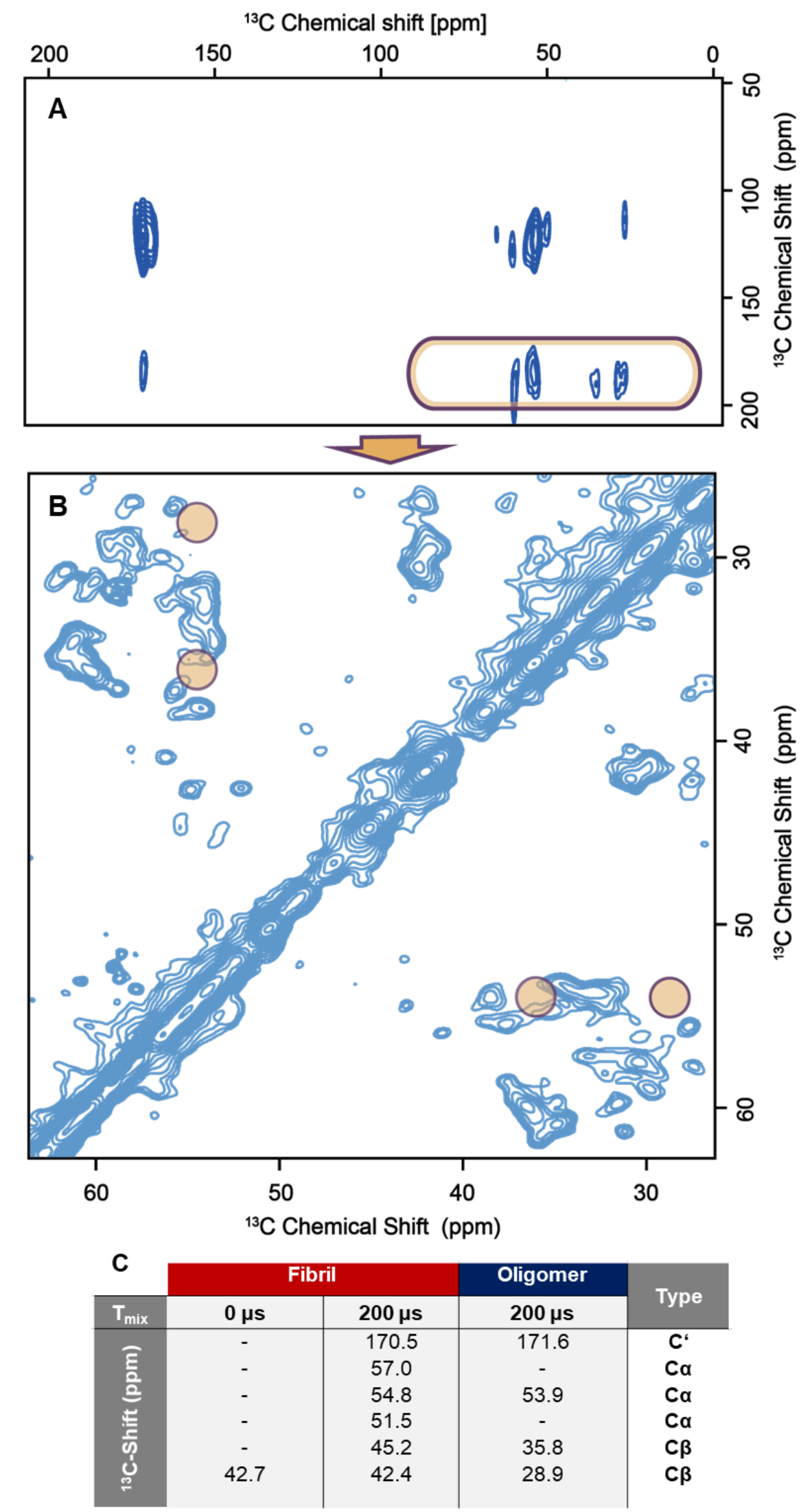

Figure 3.3.8 A) $\mathrm{NHHC}$ of ${ }^{13} \mathrm{C}$ oligomers with ${ }^{15} \mathrm{~N}$ anle138b: B) DARR spectrum of I-1 with marked regions for the observed correlations in NHHC C) Chemical shifts of observed correlations in $\mathrm{hNHHC}$ between $15 \mathrm{~N}$ of anle and $13 \mathrm{C}$ of fibrils and oligomers; 


\subsubsection{Photoaffinity labeling studies on aS aggregates}

Despite the successful characterization of the binding of anle138b to $a S$ aggregates by ssNMR, site-specific information on the interaction was not obtained. In order to achieve this, crosslinking experiments on derivatives of anle138b were carried out. For this purpose, several derivatives carrying azide functionalities (Figure 3.3.9 were synthesized by Kris Runge (MPI for Biophysical Chemistry, Göttingen, Germany).<smiles>Brc1cccc(-c2cc(-c3ccc4c(c3)OCO4)[nH]n2)c1</smiles>

anle138b<smiles>Nc1cc(Br)cc(-c2cc(-c3ccc4c(c3)OCO4)[nH]n2)c1</smiles>

krru90<smiles></smiles>

krru133

Figure 3.3.9: Chemical structures of crosslinking derivatives and anle138b for comparison

The photosensitive nature of the azide groups allows their activation by UV-radiation, followed by a reaction with suitable groups within the protein species of interest. Such photoaffinity studies have been carried out on a multitude of targets, usually for target and binding site identification. ${ }^{[177]}$ Typically mass spectrometric analysis of the labelled protein after proteolytic digestion yields residue specific information of the interaction of protein and ligand. A study on structurally similar compounds was recently carried out on aS fibrils. ${ }^{[175]}$ The workflow adapting these principles used in this work are shown in Figure 3.3.10. ${ }^{[178]}$
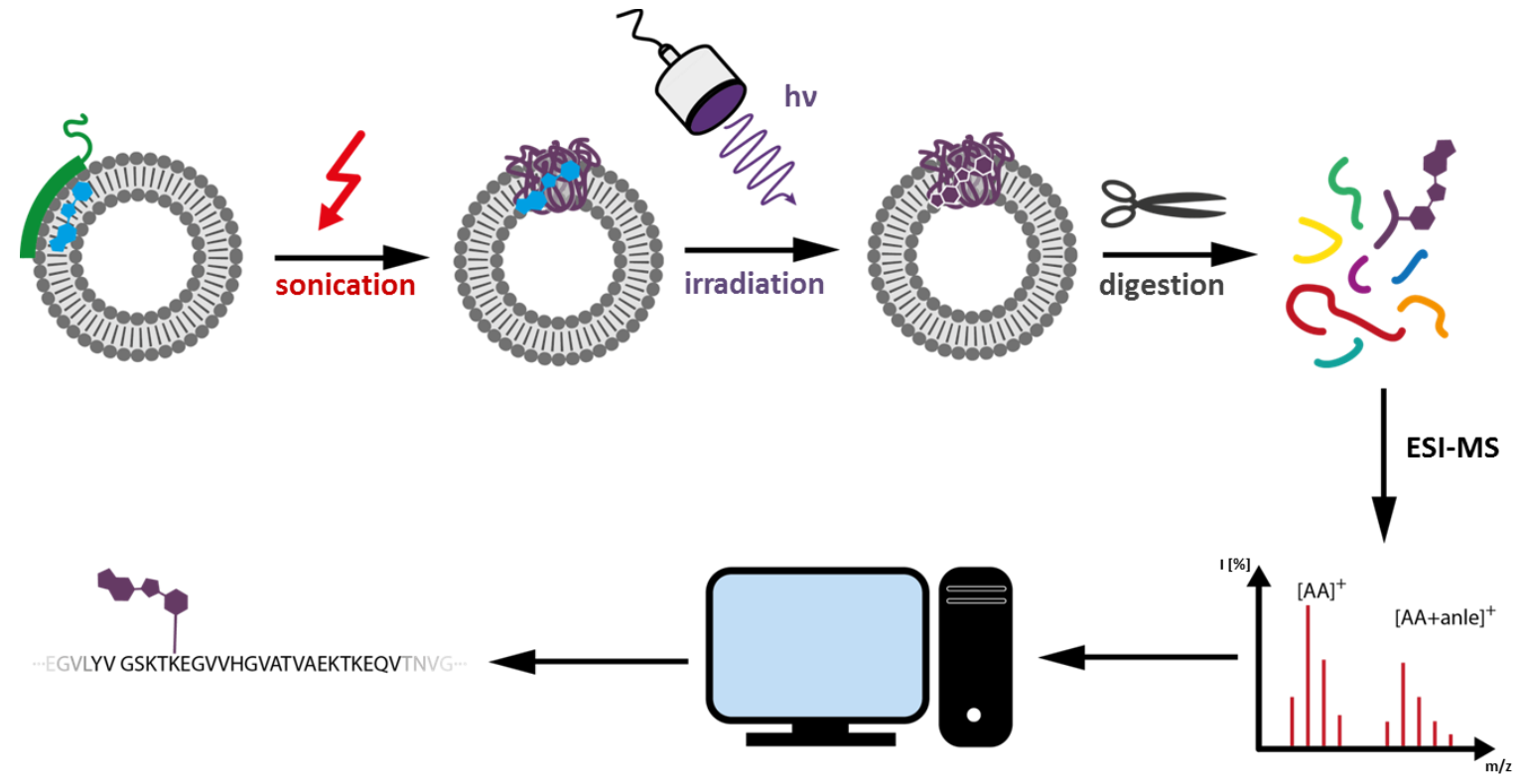

Figure 3.3.10: Strategy applied for photoaffinity studies (figure inspired by Tran et al., BBA 2016).[178] Monomers of aS were incubated with SUVs containing cross-linking derivatives of anle138b under PMCA conditions. At several points in time, samples were taken and irradiated by UV-light, followed by tryptic digestion. Protein fragments were analyzed by ESI-MS. Data evaluation was done by open search. 
Vesicles of DOPC and DOPC (7:3) containing thre crosslinking compounds were incubated with aS under PMCA conditions and aggregation was tracked by ThT fluorescence. For reasons outlined earlier, various L/P ratios were tried in preparation for the crosslinking assays, to asses the reaction kinetics (Figure 3.3.11). At a L/P ratio of 80 , aggregation of $\alpha \mathrm{S}$ is slowed down significantly, while at a ratio of 160 it is apparently stopped in the observed time window. Since at a L/P ratio of 40 binding to the lipid bilayer is expected to be strong, while aggregation is still enhanced, displaying lag times of under $12 \mathrm{~h}$, it was chosen for further studies. As the compounds are embedded in the membrane, strong protein-lipid interaction is imperative to achieving sufficient cross-linking.

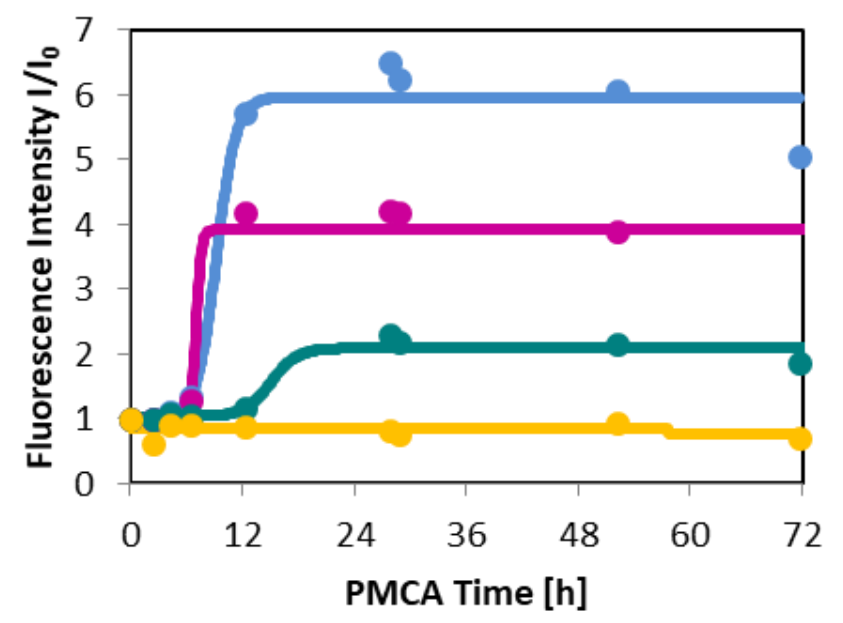

Figure 3.3.11: Kinetics under conditions for crosslinking; LP = 20 (blue), 40 (orange), 80 (green), 120 (yellow)

For photoaffinity studies aliquots were taken from aggregation mixtures at various points in time and subjected to UV radiation. The irradiated samples were proteolytically digested by Trypsin after precipitation with Acetone and analyzed by ESI Mass spectrometry in the group of Henning Urlaub (MPI for Biophysical Chemistry, Göttingen, Germany). Experiments on monomeric protein were carried out in the presence of both krru90 and krru133, while experiments on aggregated protein included krru133 exclusively.

In the analysis of the MS data two mass adducts, are justified by rearrangement of the photoactive compounds and subsequent reaction with protein sidechain, show up at $m / z=277.09$ and $m / z=263.07$. The ratio of the corresponding peak intensities from the modified to the unmodified protein yields an estimate of the affinity of the compounds for the modified sequences. Open search analysis resulted in the identification of several labelled Lysine residues along the sequence of aS (Table 3.3.1). The only exclusion from this is the labeling of H50. This apparent preference of krru90 and krru133 for nucleopilic reactants indicates a reaction mechanism that progresses via a ketenimine intermediate. ${ }^{[179,180]}$ The 
most likely reason for a preference of this mechanism is the stabilization of intermediates via the extended $\pi$-system between the aromatic rings of the compounds. ${ }^{[181,182]}$

The highest labeling efficiency in monomeric aS is observed for $K 12, K 21, H 50$ and $K 60$, while minor affinities are observed for the pairs K32/K34, K43/K45 and K96/K97 and residue $K 80$. Due to their proximity, neighboring Lysines are not efficiently distinguished, so that K23 and $\mathrm{K} 58$ are treated as labeled residues in the following. Upon aggregation labeling efficiences apparently drop, as the measured intensity ratios decrease across all identified labeling sites. In the case of oligomeric species, the most notable difference to monomeric aS is the increase in affinity towards $\mathrm{K} 32 / \mathrm{K} 34$. In addition labeling of $\mathrm{K} 43 / \mathrm{K} 45, \mathrm{~K} 80$ and K96/K97 is no longer observed. Taking into account the low affinity in monomer and the decreased ratios for aggregates in general, it might be, that labeling of these residues is simply not detected. Upon fibrilization the increased affinity for K32/K34 seems conserved, while labeling of K21/K23 is not observed at all in contrast to oligomer and monomer. The absence of labeling for $\mathrm{K} 80$ is consistent with the results on oligomers. Unlike for oligomeric aS however, fibrils are labeled at residue K43/45 and K96/K97 respectively.

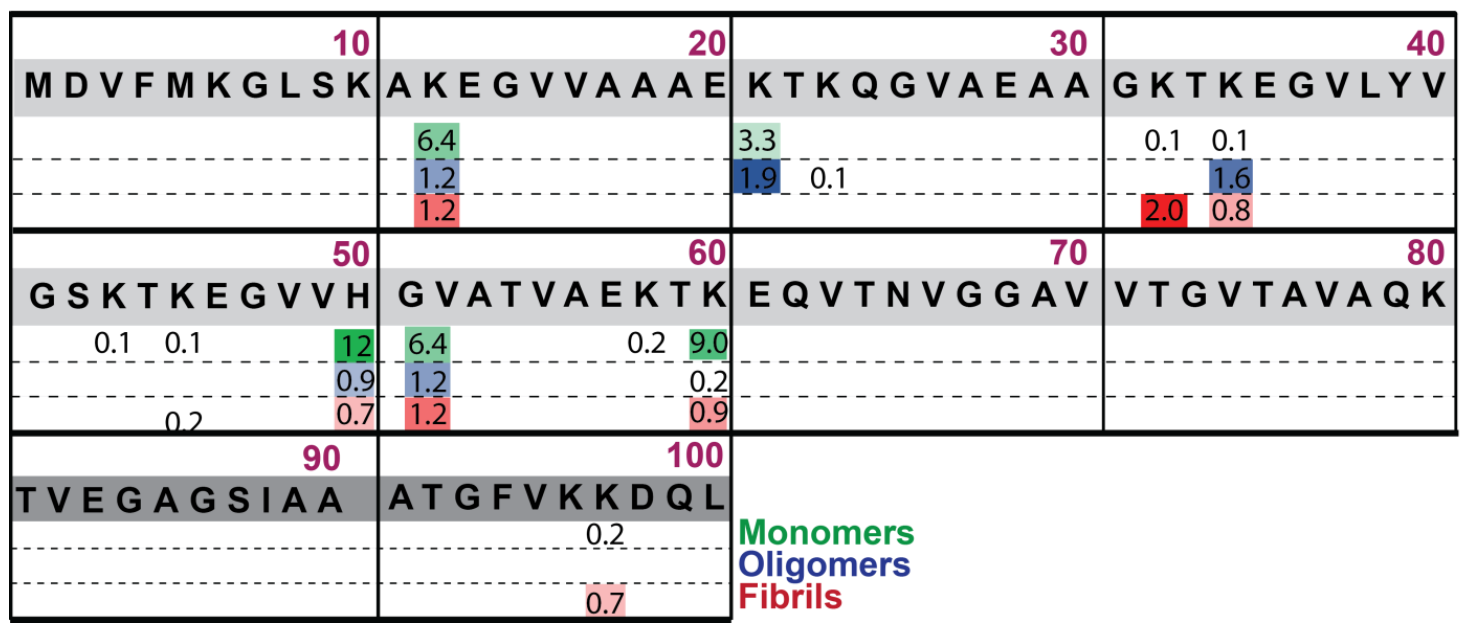

Table 3.3.1: Contacts found in open search using MaxQuant on ESI-MS data from photoaffinity experiments with krru133 on monomers (green), oligomers (blue) and fibrils (red); values represent the ratio of the mass fragment $\Delta m / z=277.09$ between modified and unmodified protein averaged over all experiments (if a contact was not found, the ratio was included as 0 ). High values indicate strong modification; low values indicate weak modification or statistically less relevant modifications. 


\subsubsection{Molecular Dynamics Simulations}

Building on the successful identification of potential binding sites in NMR, MD simulations were carried out by Dirk Matthes (MPIBPC, Göttingen, Germany) to further the understanding of the atomistic details of anle138b interactions with aS aggregates. For this purpose, several models were derived from the X-ray structure coordinates of various aS fibril conformations deposited in the Protein Data Bank (PDB) (Figure 3.3.12). These models include the cryo-EM and NMR-derived "Greek-key" containing polymorph 1 (6CU7, 2NOA) $)^{[97}$, ${ }^{101]}$ and polymorph $2(6 \mathrm{RTO})^{[100]}$, as well as a basket-shaped nonamer consisting of three sets of three identical $\beta$-hairpins of the peptide $\alpha S_{33-58}(5 \mathrm{~F} 1 \mathrm{~T})$ (Figure 3.3.17). The latter structure was found by Salveson et al. through crystallographic methods. ${ }^{[183]}$ For models of fibril structures, stacks of five cross $\beta$-strand layers were simulated either as single (5mer) or paired strands (10mer).
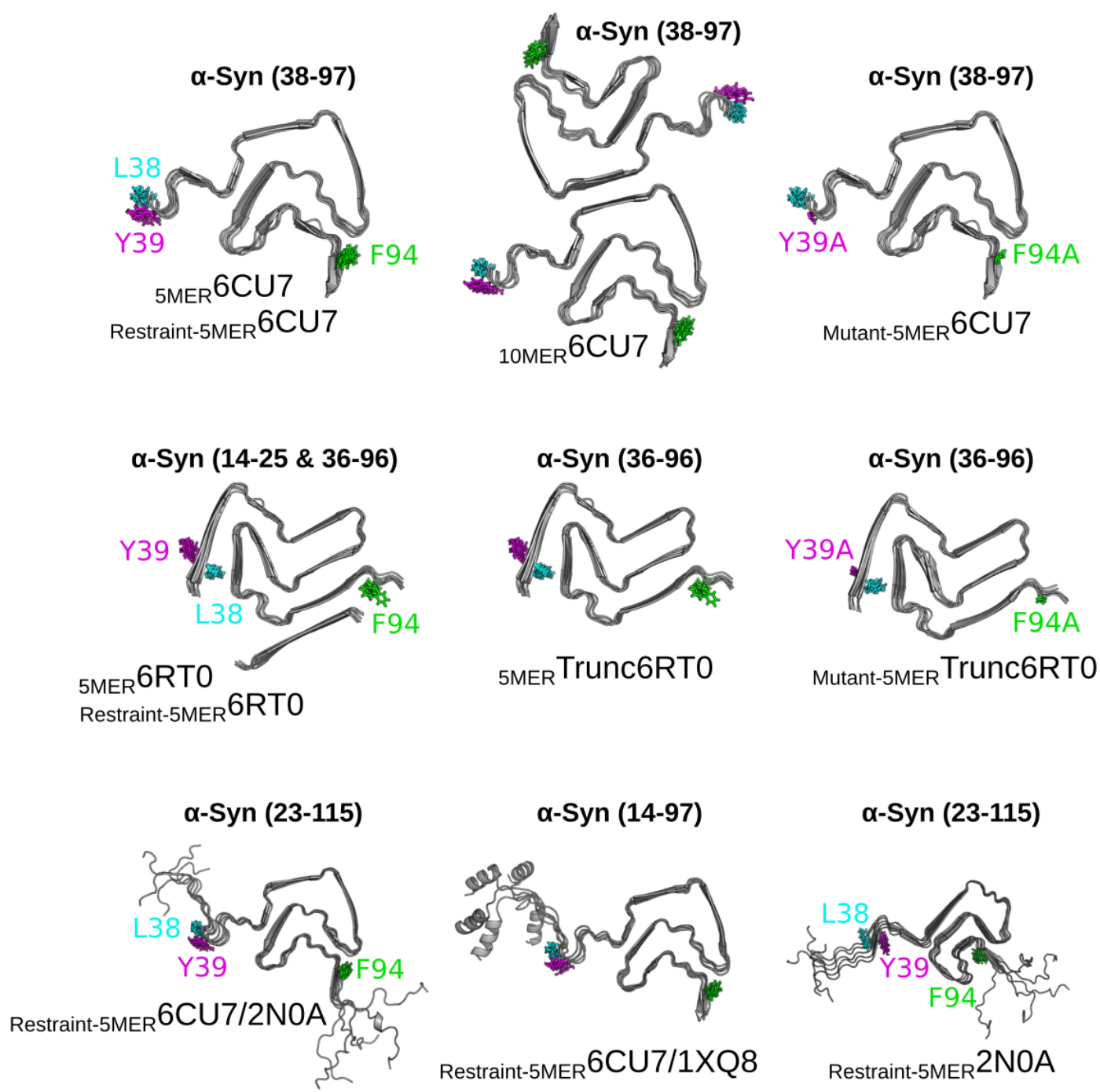

Figure 3.3.12: Models of $\alpha S$ fibrils used for MD simulations on anle138b interaction; structures were derived from PDB deposited data determined by cryo-EM (6CU7, 6RT0), ssNMR (2NOA) and solution NMR (1XQ8). 
For each model, 50 independent simulations were carried out in order to get a statistical quantification of the binding process. Within the simulated time scale of $250 \mathrm{~ns}$ anle $138 \mathrm{~b}$ is able to undergo multiple unbiased binding events. However, as expected for this highly hydrophobic molecule, once anle138b gets into proper contact with the protein assemblies, unbinding events are rarely observed.

A
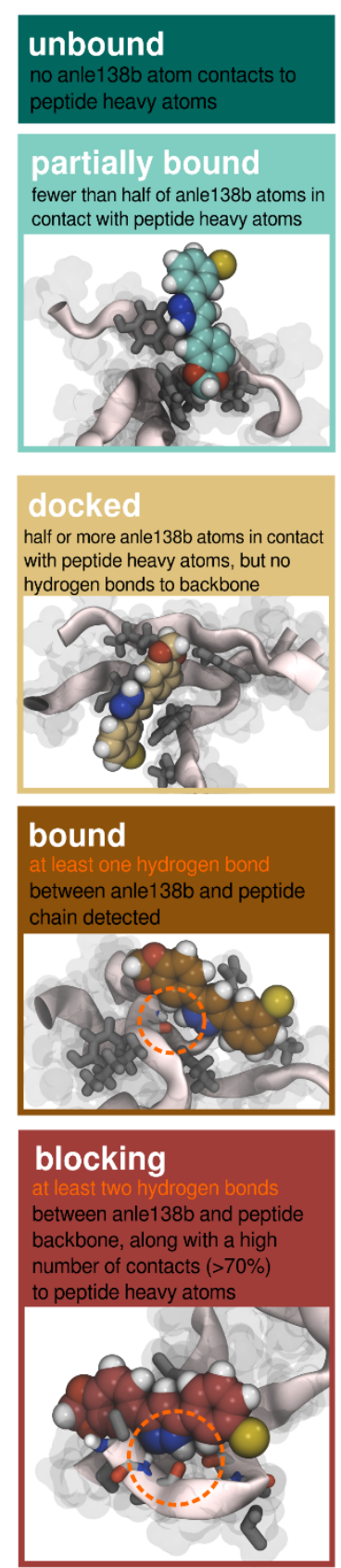
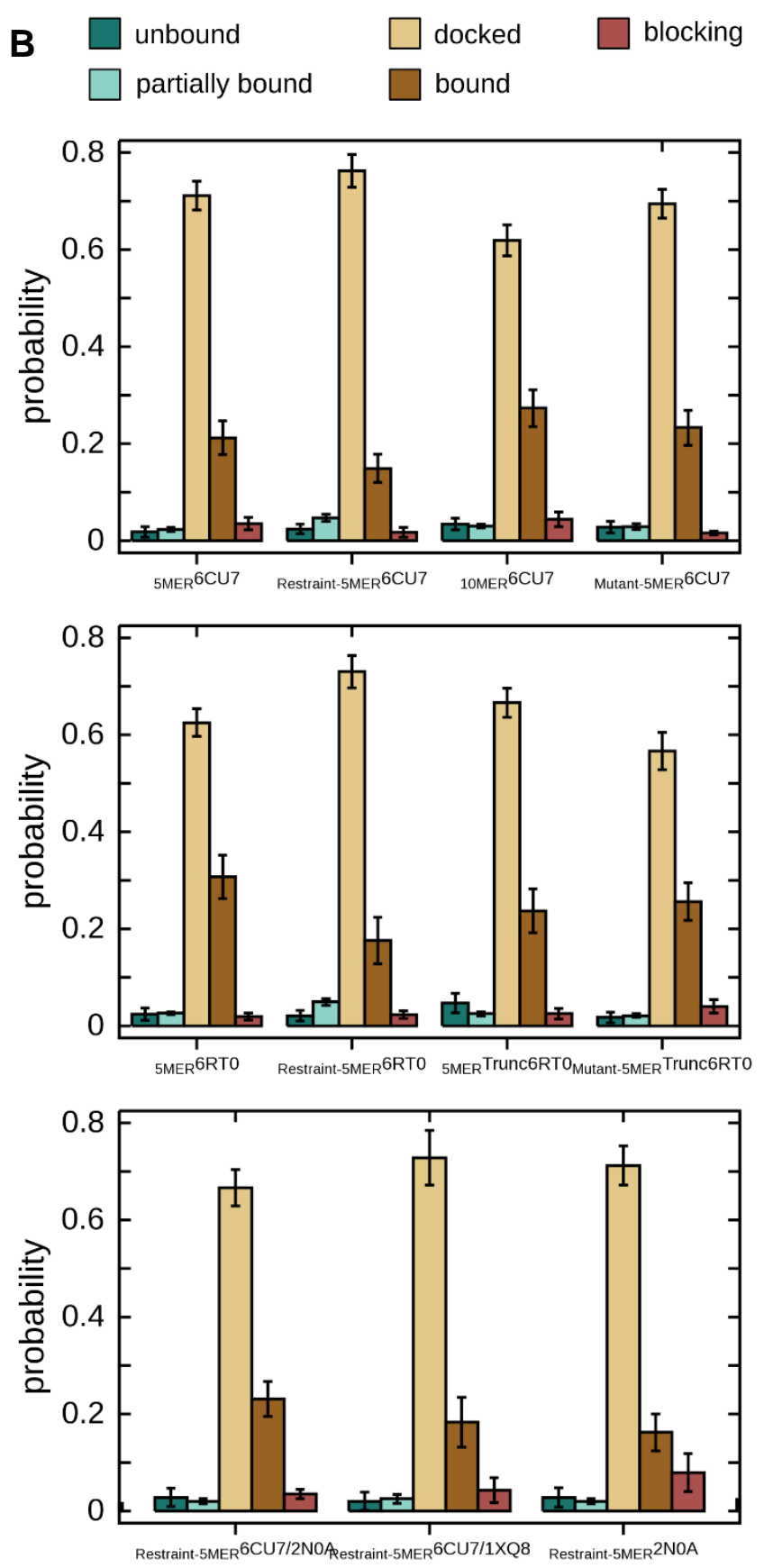

Figure 3.3.13: Contact probabilities of anle138b binding to aS fibrils broken down into binding modes; A) Representative simulation structures analogous to Matthes et al. Chem. Neurosci. 2017[139]; B) Population of the individual binding modes in A. 
This general tendency is reflected in the small portion of unbound anle138b molecules (Figure 3.3.13) seen in the second half of the trajectories (as used for all subsequent analyses) consistently across all simulated models.

The contact probabilities of anle138b for each residue of the simulated aS multimers are depicted in Figure 3.3.14. Across all fibril models, a high contact probability for residues Y39 and F94, as well as neighboring residues are found. In the model for 6CU7, the elevated probabilities also include V66 and G67, both of which are in close spatial proximity to F94. Phenylalanine and Tyrosine feature characteristic $\mathrm{C}_{\beta}$-chemical shifts that would justify the observed correlation at $42 \mathrm{ppm}$ in the hNHHC experiments in Figure 3.3.6. These residues are located at the edges of the ordered cross- $\beta$ core of the fibril filaments to the more disordered $\mathrm{N}$ - and $\mathrm{C}$-terminal regions.

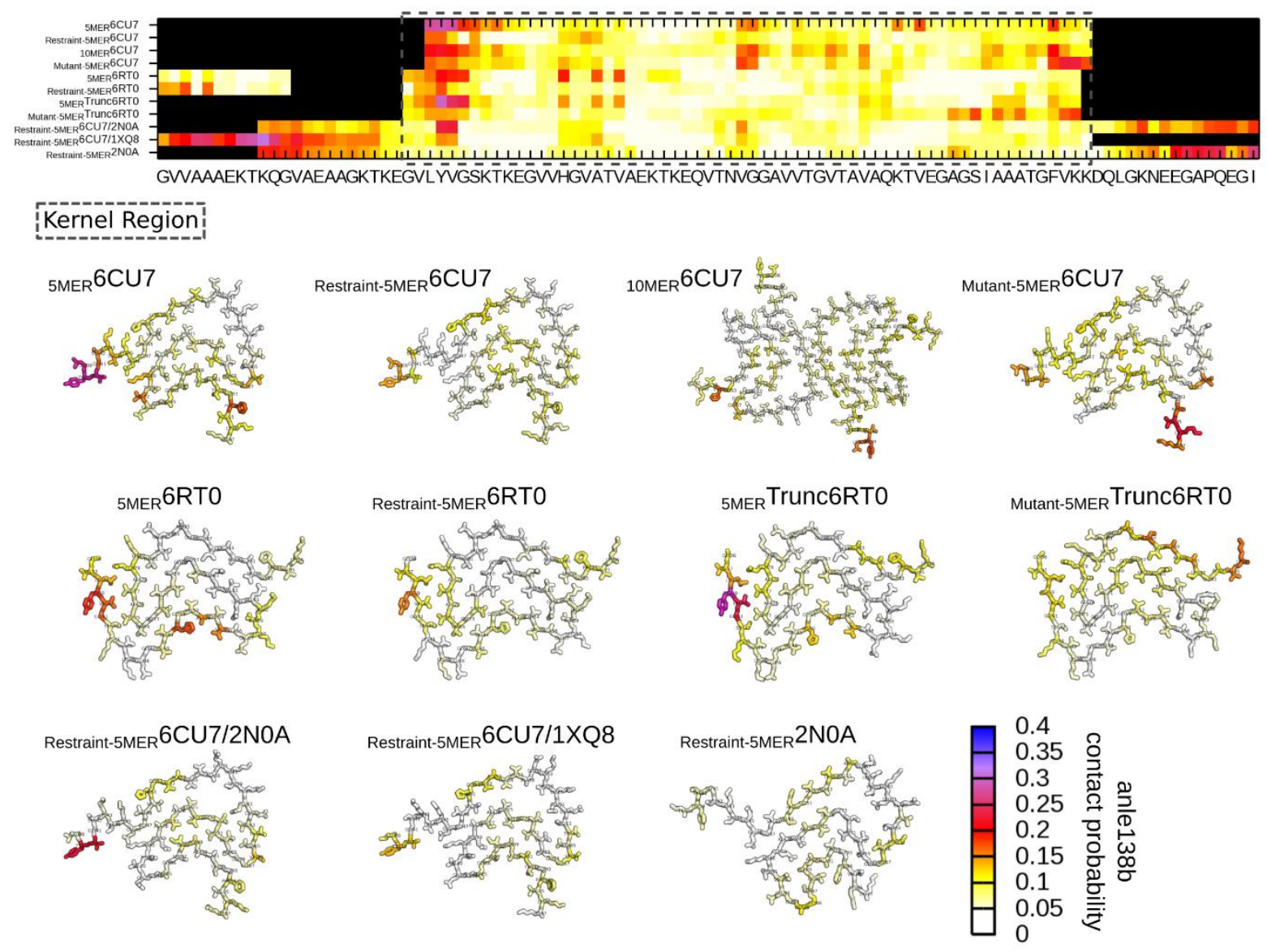

Figure 3.3.14: Contact probabilities for interaction of anle138b with residues along the aS sequence for the models Figure 3.3.12; Probabilites are averaged over 50 simulations, a contact probability of 1 is achieved if anle138b is in contact $(\mathrm{d} \leq 0.4 \mathrm{~nm})$ with all 5 similar residues in a fibril stack over all simulations. Contact probabilities were color coded and displayed on a single folded monomer for each model.

Comparing anle138b contact probabilities for the edge layers of the fibril stacks, exemplary of fibril ends, with respect to the 3 central layers, unspecific binding towards hydrophobic 
residues, especially in the sequences ${ }_{68}$ GAVVTGVTAVAQ ${ }_{79}$ and ${ }_{86}$ GSIAAAT $_{92}$, are observed (Figure 3.3.15). In the paired filament model of $6 \mathrm{CU} 7$, increased anle138b binding is also additionally observed to the hydrophobic residue sequence ${ }_{51} G_{V A T V A E}$, which forms the interface in the decamer.
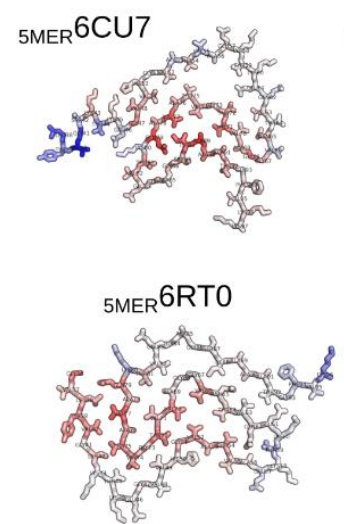

Restraint-5MER $6 \mathrm{CU} 7 / 2 \mathrm{NOA}$

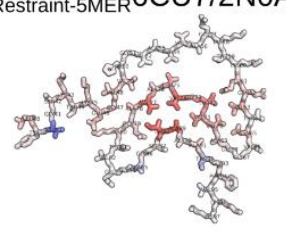

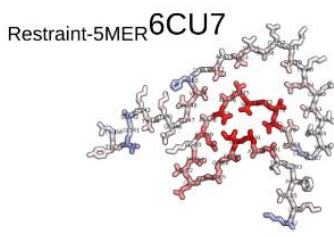

Restraint-5MER 6RT0

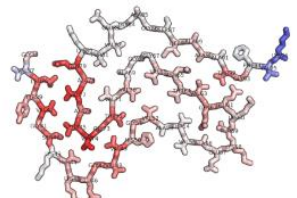

Restraint-5MER 6CU7/1XQ8

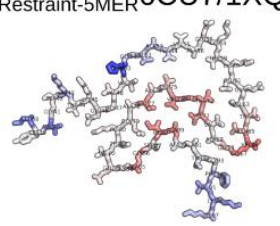

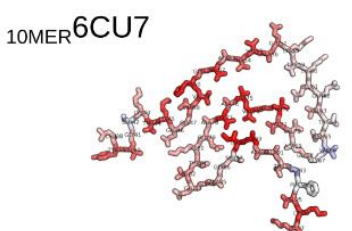

5MERTrunc6RT0
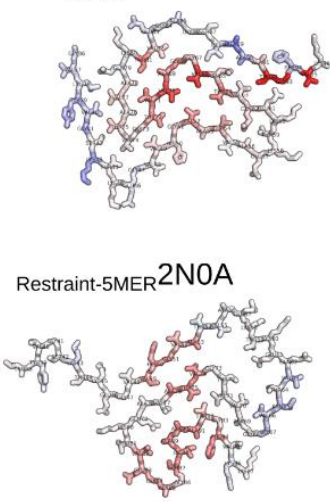

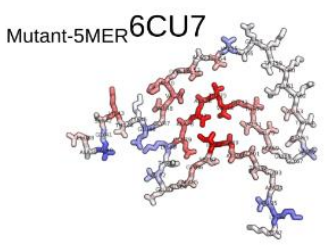

Mutant-5MER Trunc6RT0
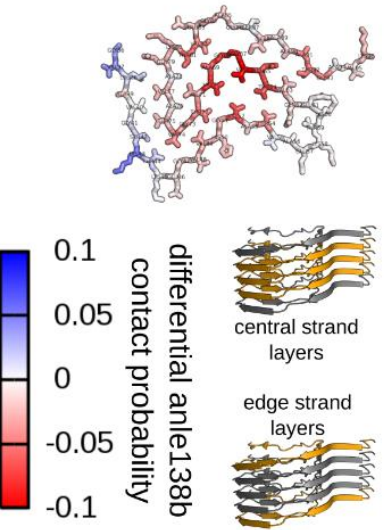

Figure 3.3.15: Differential binding of anle138b to central and edge layers of the simulated fibril stacks; blue: high contact probability in central layers; red: high contact probability in edge layers.

The obvious preference of anle138b for residues located at the edges of the fibril core, led to the use of extended models, to probe whether the compound binds unspecifically to the disordered or solvent exposed protein backbone. The 5mer of 6CU7 was therefore extended by 15 residues in random coil conformation taken from the NMR aS fibril structure 2NOA towards both the $\mathrm{N}$ - and $\mathrm{C}$-terminus to yield a model spanning residues $23-112$. Additionally the 5 mer of $6 \mathrm{CU} 7$ was extended by 24 residues taken from the mostly helical membrane bound aS conformation $(1 \mathrm{XQ8})^{[184]}$ towards the $\mathrm{N}$-terminus to yield a model spanning residues 14-97. Indeed, contact probabilities were lowered in favor of residues 24-33, 101112 (6CU7/2NOA) and 14-33 (1XQ8), however values for the binding site around Y39 and F94 was still elevated compared to the rest of the sequence.

In addition to the two structures determined by cryo-EM, simulations also included the full NMR structure 2NA0. Although the overall geometry of this structure is in agreement with 6CU7, several residues feature a different orientation. These difference lead to a hydrophobic pocket, comprising the residues ${ }_{86}$ GSIAAATGFV $_{95}$, located in an outside kink of the Greek key motif. Simulations show, that there is no preferred binding to this site, probably 
due to lack of solvent accessibility, even though an opening of this dense pocket is not expected. To preserve the respective fibril conformation of the elongated models over the course of the simulations, the kernel region of the fibril structures was subject to position restraints in all three cases.

To further probe the influence of protein dynamics on the compound interactions, several simulations with fully position restrained fibril stacks of original 6RT0 and 6CU7 models were carried out. In these models, lacking the extensions, contacts to the fibril ends were found to spread more evenly across the sequence (Figure 3.3.15), with less specific binding to $Y 39$ (Figure 3.3.14). In 6RTO the sequence ${ }_{50} \mathrm{HGVATV}_{55}$ forming a kink shows a decreased binding, while in 6CU7 also a minute preference for F94 is found.

The preference of anle138b for the aromatic residues Y39 and F94, especially when offered interactions with more flexible backbone conformations is quite remarkable, but ties together with the observed preference for the disordered termini in the elongated models (Figure 3.3.16). The high residue solvent accessibility (SASA) and exposed peptide backbones of disordered conformations are suitable for deep burial of anle138b and help explain the favorable binding in the simulation ensembles (Figure 3.3.16).

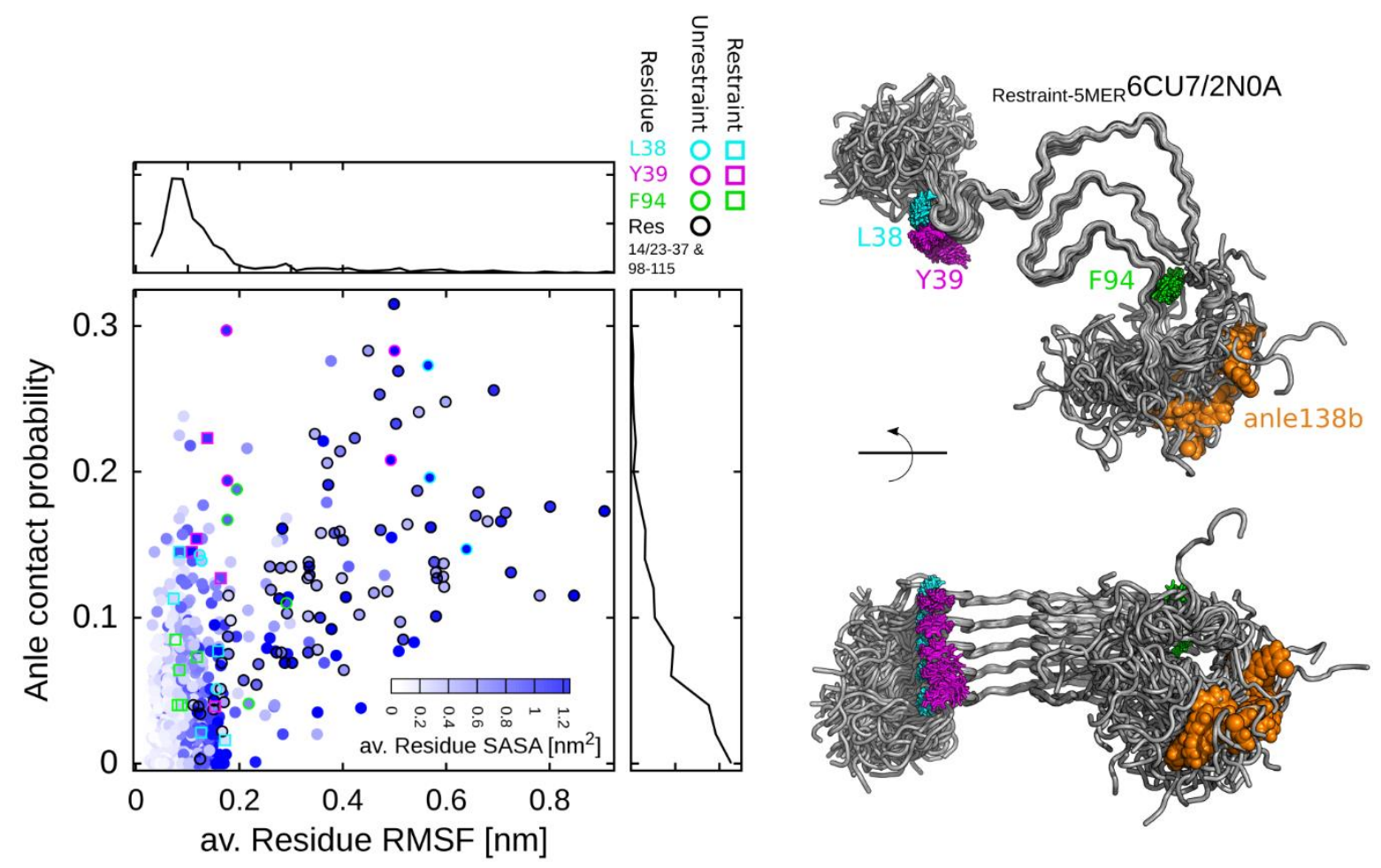

Figure 3.3.16: Influence of position restrains on the binding of anle138b to fibril models. Contact probabilities were plotted against the RMSF, indicating fluctuations in structure. Contacts were color coded for solvent accessibility (SASA) of the residue in contact. Contacts to L38 (cyan), Y39 (pink) and Y94 (green), were highlighted for unrestrained (circle) and restrained (square) models. 
To examine, whether also aromaticity is imperative to explain the regiospecific binding of anle138b, both residues were mutated to alanine in the 6CU7 and truncated 6RT0 model. In both instances strong binding to exactly these sites is lost, giving rise to the hypothesis, that $\pi-\Pi$ stacking has a major contribution in the binding interaction of anle138b to protein aggregates.

MD simulations of the basket-shaped nonamer yield a less conclusive set of regiospecific contacts, with probabilities spread over the whole sequence. A reason for this is found in the small size of the highly homologous and non-fibrillar oligomer. The lack of the repeated residue packing symmetry, as found in mature fibril conformations, is causing anle138b to come in close proximity to many residues simultaneously, upon binding to its preferred site. Elevated probabilities are seen for L38, V40 and V49, whereas Y39 which is buried in the oligomer core is least accessible to anle138b contacts (Figure 3.3.17).
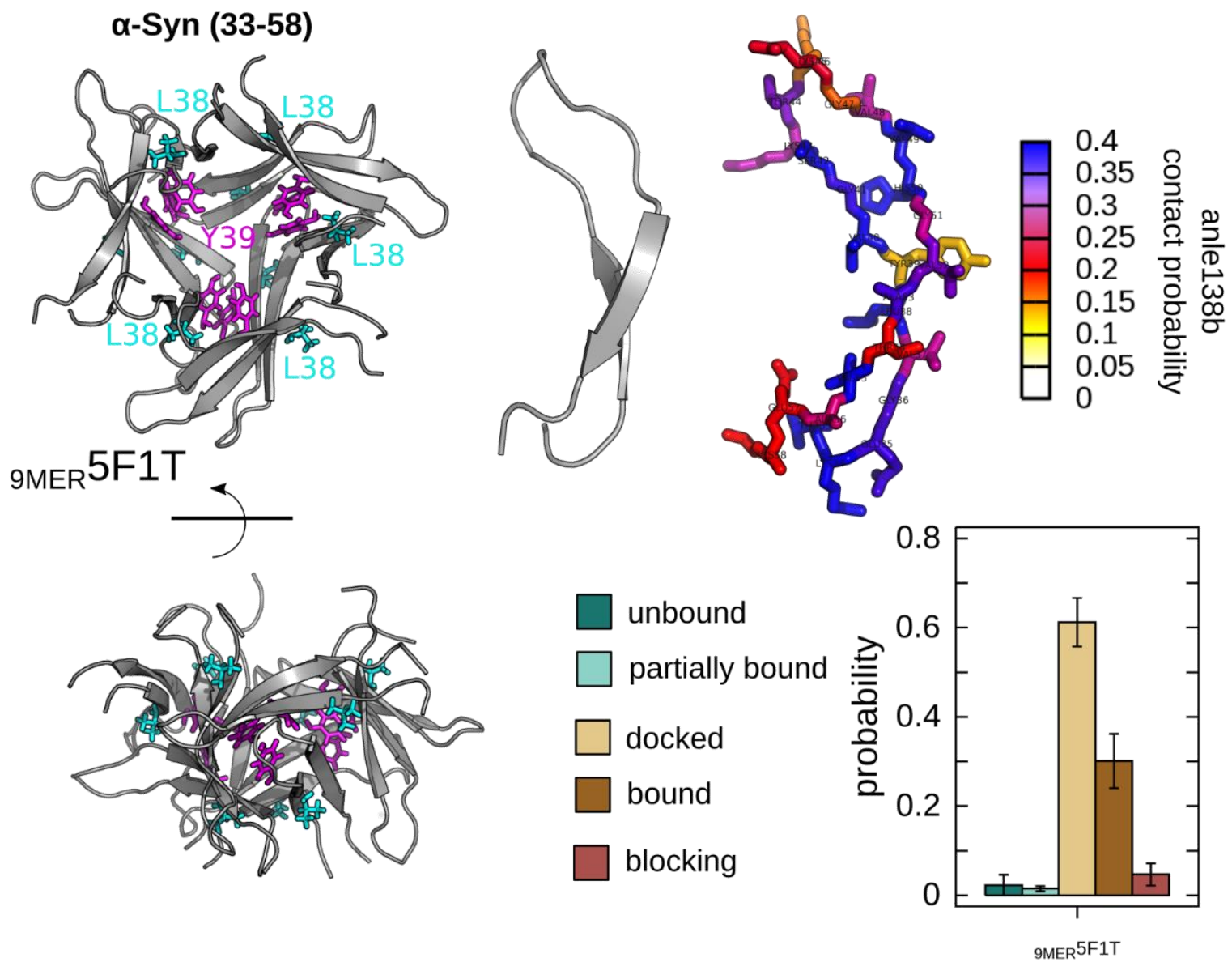

Figure 3.3.17: MD-simulations on the interaction of anle138b with the basket shaped oligomer by Salveson et al.[183] consisting of 9 identical $\alpha \mathrm{S}_{33-58}$ antiparallel $\beta$-hairpins. Top right: Color coded contact probabilities projected on a single monomer fold; Bottom right: Binding modes of anle138b to the peptide analogous to Figure 3.3.13. 


\section{Discussion}

\subsection{PMCA drives the formation of membrane bound aS oligomers}

To the best of my knowledge, the present study is the first account of biophysical studies on the aggregation of $\alpha S$ in the presence of phospholipids under PMCA conditions. It has recently been shown, that de novo aggregated seeds produced by PMCA from M83 mouse brain, cause accelerated disease progression after cerebral inoculation into young mice. ${ }^{[185]}$ M83 express the human aS carrying the A53T mutation, which has been shown to have a high propensity to form oligomers in vitro. ${ }^{[186]}$ An earlier study on PrP showed the production of toxic $\beta$-sheet rich oligomers by PMCA, leading to the apoptosis in cortical neuron cells. ${ }^{[187]}$ The increased interest in PMCA regarding detection and amplification of $\alpha S$ aggregates from patient material such as brain homogenates, makes the understanding of lipid bound aggregation under these conditions relevant. ${ }^{[123]}$

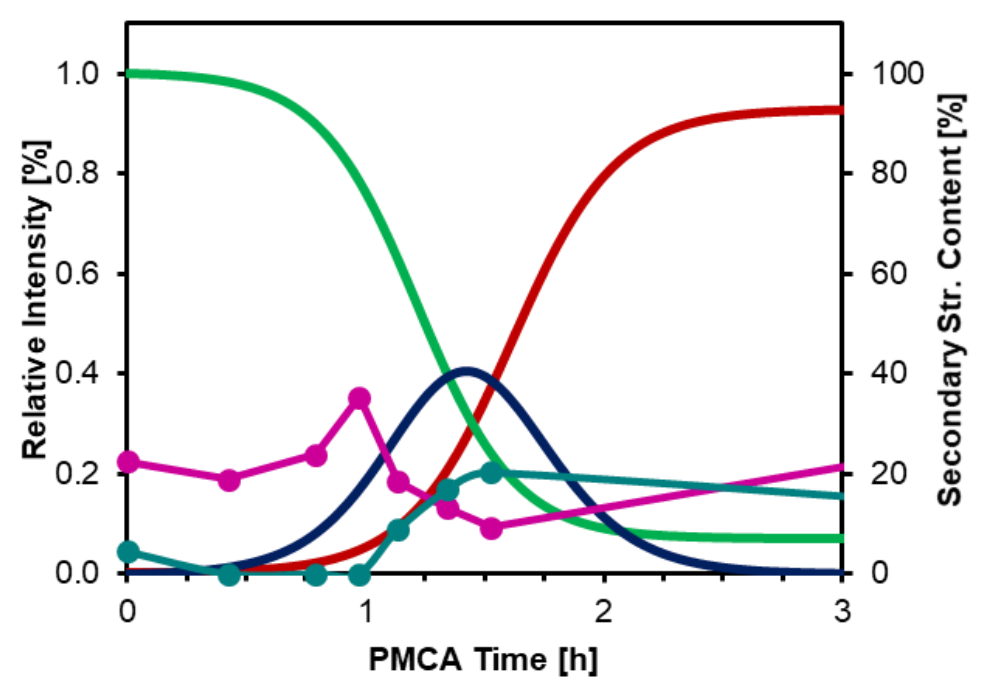

Figure 4.1.1: Combination of kinetic data. Monomer (green) and fibril (red) and estimated oligomer concentration, derived from solution NMR and ThT as well as anti-parallel (pink) and parallel (teal) $\beta$-sheet content of $\alpha S$ during aggregation under PMCA conditions in the presence of phospholipids.

A striking finding of the use of PMCA in this context is the large amount of oligomers that builds up in the aggregation process (Figure 4.1.1). The levels of oligomeric species estimated from NMR and ThT peak at $40-60 \%$, which is significantly higher than the commonly perceived levels, which are below $10 \%$ of the initial monomeric solution. ${ }^{[17]} \mathrm{A}$ study using small angle X-Ray scattering (SAXS) found similar levels of oligomers to the ones reported here $(30-40 \%)$, albeit in the absence of phospholipids. ${ }^{[188]}$ In contrast, Cremades et al. found oligomer fractions of $<5 \%$, using sm-FRET. ${ }^{[189]}$ By means of gel filtration, Lashuel et al. determined a maximum amount of $15 \%$ for oligomeric aS bearing 
various familial mutations. ${ }^{[190]}$ It has been argued however, that the use of gel-filtration or size-exclusion, aiming at separation of oligomers from monomers might cause a shift in equilibrium leading to the dissociation of aggregates. ${ }^{[191]}$ This phenomenon has been shown to proceed under super-cooled conditions for fibrils ${ }^{[93]}$ and was later confirmed under resting conditions at $37^{\circ} \mathrm{C} \cdot{ }^{[189]}$

Comparing the time traces with those obtained from CD (Figure 4.1.1), it can be seen, that anti-parallel $\beta$-sheet content rises just before fibril formation. By this time ThT fluorescence is negligible and monomer concentration has dropped to about $25 \%$ and the estimated oligomer concentration is about $15 \%$. The disparity originates from poor fitting of the ThT data. Assuming, that the maximum in anti-parallel $\beta$-sheet content also marks the maximum oligomer concentration, which is reasonable given the sharp rise in fibril formation from here on out, the levels in oligomer are still higher than in most previously reported protocols.

The significant difference to the published levels of oligomeric aS demands an explanation of the underlying mechanisms during PMCA. Since the accumulation of intermediates prior to fibrillization were neither reproduced in the absence of phospholipids, nor under other agitation conditions, the influence of sonication on the phospholipid membranes must be considered.

Ultrasonic waves cause the rapid expansion and collapse of gas bubbles in water, a process called acoustic cavitation. ${ }^{[192]}$ The hydrophobic surface of these gas bubbles is thought to adsorb protein molecules and the increase in local concentration, paired with the large pressure and temperature changes ensuing cavitation, trigger amyloid formation. ${ }^{[193]}$ This process is significantly enhanced in the presence of phospholipids.

As a result of the membrane affinity, the local concentration of $\alpha S$ is strongly enhanced, leading to increased aggregation rates at low L/P ratios even in quiescent conditions. ${ }^{[109]}$ At $\mathrm{L} / \mathrm{P}$ ratios of 10 , which was used in our aggregation assays, approximately 500 aS molecules bind to one SUV (see materials and methods section for calculation). Studies on GFPlabelled aS estimated the amount of monomer per vesicle to about 70 under physiological conditions. ${ }^{[194]}$ Increasing the lipid content increases the number of SUVs while at the same time the number of $\alpha S$ molecules per SUVs does not increase. Rather, aS seems to populate the vesicle surface until free monomer is depleted. At a L/P ratio of about 20 this still results in strong aggregation, but the amount of $\alpha S$ in solution has been reduced to about $20 \%$ of the total protein, so that aggregation is slower than at $L / P=10$. Increasing the lipid content eventually dilutes monomers on the membrane surface and free monomer so much, that fibril formation is slowed down or inhibited. Tensiometry derived data showed that an amphipathic helix takes up about $0.15 \mathrm{~nm}^{2}$ per residue, resulting in an area of $15 \mathrm{~nm}^{2}$ for aS on 
phospholipids. ${ }^{[195]}$ This would mean, that under the conditions presented here, vesicles are fully covered by aS.

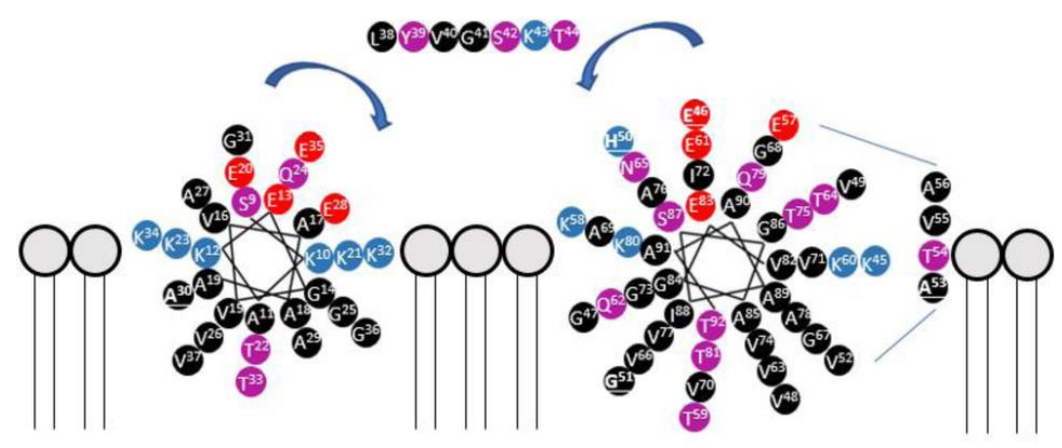

Figure 4.1.2: Helical Wheel of aS monomer embedded in phospholipid membranes, showing the orientation of hydrophobic residues towards the bilayer core. ${ }^{[196]}$

The availability of hydrophobic surfaces alone still does not fully explain the observed tendency to form oligomers, given the absence under shaking conditions. The pressure changes and cavitation during sonication cause disruption of lipid bilayers, a process called sonoporation. ${ }^{[197]}$ This effect has been successfully studied regarding its potential in drug delivery through membranes. ${ }^{[198]}$ Binding of aS has been shown to be dependent on the presence of membrane defects, as it binds to SUVs but not LUVs or GUVs of zwitterionic lipids. ${ }^{[199]}$ It has been hypothesized that this behavior arises from the insertion of the amphipathic helix of the protein into the defect, modulating the lateral pressure of the outer leaflet. ${ }^{[200]}$ In this context, aS has been linked to membrane thinning. ${ }^{[201]}$ These factors make it very likely to be in the vicinity of a sonication-induced pore. The exposure of hydrophobic lipid tails to the membrane interior could be a driving force for misfolding of aS (Figure 4.1.2).

Probing the effect of focused ultrasound on lipid membranes Man et al. employed MDsimulations, revealing that pores in the bilayer formed under high local pressure changes are stable on a $\mu$ s time scale, albeit for a non-curved bilayer. ${ }^{[202]}$ Such a pore would be open long enough to allow formation and insertion of disordered loops $(0.1-0.3 \mu \mathrm{s})$, although not for the formation of $\beta$-sheets $(5-20 \mu \mathrm{s}) .{ }^{[203]}$ This is in line with our observation, that the first observed structural features in $\alpha S$ intermediates are two loops around T59 and V74/T75 respectively. Misfolded proteins formed at the edges of the pores are likely to recruit other close by aS molecules, resulting in amorphous aggregates. An in-depth discussion of the ensuing structural implications follows in chapter 4.3.

This mechanism is supported by the complete conversion of membrane bound aS into ThTnegative aggregates by $t_{\text {lag }}$ as detected by ${ }^{1} \mathrm{H}-{ }^{15} \mathrm{~N}-\mathrm{HSQC}$ (Figure 3.1.3), which is not seen during orbital shaking (Figure 3.1.4). Despite the lag time being twice as long under the latter 
conditions, the rate of fibril formation is comparable to that under PMCA and final ThT fluorescence is even higher. It seems therefore that elongation of the initially formed nuclei is unchanged. The reduced ThT response might be due to breaking of the fibrils through sonication and subsequent entanglement of the small fragments.

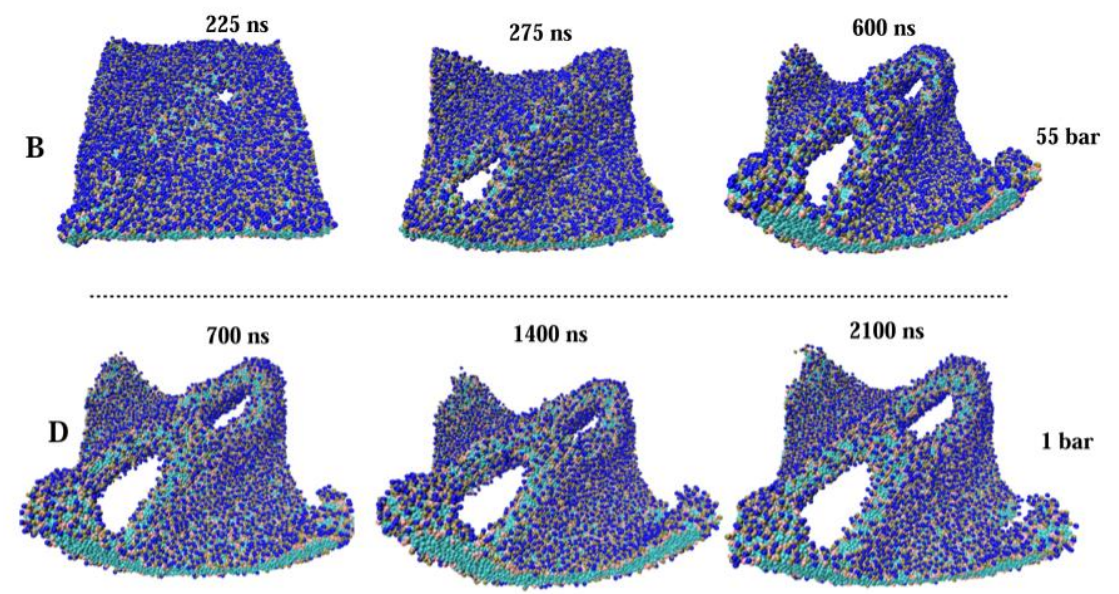

Figure 4.1.3: Snapshots of lipid membranes simulated by Man et al.; A: pore formation at high focused ultrasound intensity; B: equilibrium simulation on pores at ambient pressure.[202]

Furthermore, the substantial oligomer build-up has a kinetic component. Conversion of monomers into oligomers and subsequent nucleation involves several energetically unfavorable steps, resulting in a slow reaction reflected in the lag time. ${ }^{17]}$ Oligomer populations are hence low throughout the reaction, since once they are formed, their conversion into larger aggregates is usually faster than their formation. In the preparation presented here, misfolded oligomers are formed in bulk, followed by subsequent fibril formation, an observation clearly in favor of nucleated conformational conversion. ${ }^{[204]}$ The successive formation of structured regions between $\mathrm{I}-1$ and the final fibril supports this idea. In a nucleated polymerization model, in which monomers form independent nuclei, the structural features of intermediates are expected to resemble that of fibrils. ${ }^{[15]}$

A fundamental observation made during the observation of aS under various conditions, is that the monomer concentration of $\alpha S$ is non-zero in the majority of cases. It is often assumed that fibril formation is irreversible and that the pleateau region represent complete conversion. ${ }^{[205]}$ However other studies have similarly shown, that upon fibril formation, residual monomer can be found. ${ }^{[206]}$ This in in line with the general observation that fibrils can exist in equilibrium with other protein species. ${ }^{[207]}$ Neglecting monomer concentration and simplifying the analysis to a fibril formation only is therefore highly problematic and neglects the thermodynamics of the process. 


\subsection{A new aS fibril polymorph or just Polymorph 2c?}

The aS fibrils grown in this study show several extended $\beta$-sheets with distinct turns at residues V74 and T81 as well as T59. Comparison of chemical shifts to data deposited in the BMRB revealed partial structural similarities with polymorph 2 (BMRB Entry 18860), reported by Guerrero-Ferreira et. al.. ${ }^{[100,163]}$ These similarities encompass a loop region formed by residues V70-K80 and an extended $\beta$-sheet from G51-V55. However, the structures also show distinct dissimilarities, so that it is safe to say, that the structure under examination is not polymorph $2 \mathrm{a}$ or $2 \mathrm{~b}$. Nevertheless, without further studies we cannot conclude whether the present differences are enough to label this structure as a new polymorph on its own.

The recently published polymorphs $2 \mathrm{a}$ and $2 \mathrm{~b}$ only display notable differences regarding the putative salt bridges connecting their virtually identical filaments. ${ }^{[100]}$ NMR spectra of both forms were reported to be indistinguishable. In contrast, the structures of polymorphs $1 \mathrm{a}$ and $1 \mathrm{~b}$ exhibit more prominent structural peculiarities. ${ }^{[99]} \mathrm{Li}$ et al. showed, that the two polymorphs share a conserved Kernel between residues V49 and A78, and that the two polymorphs mainly differ in the interface between the filaments. Unlike polymorph 2 , the known structures for polymorph 1 feature a steric-zipper type interface.

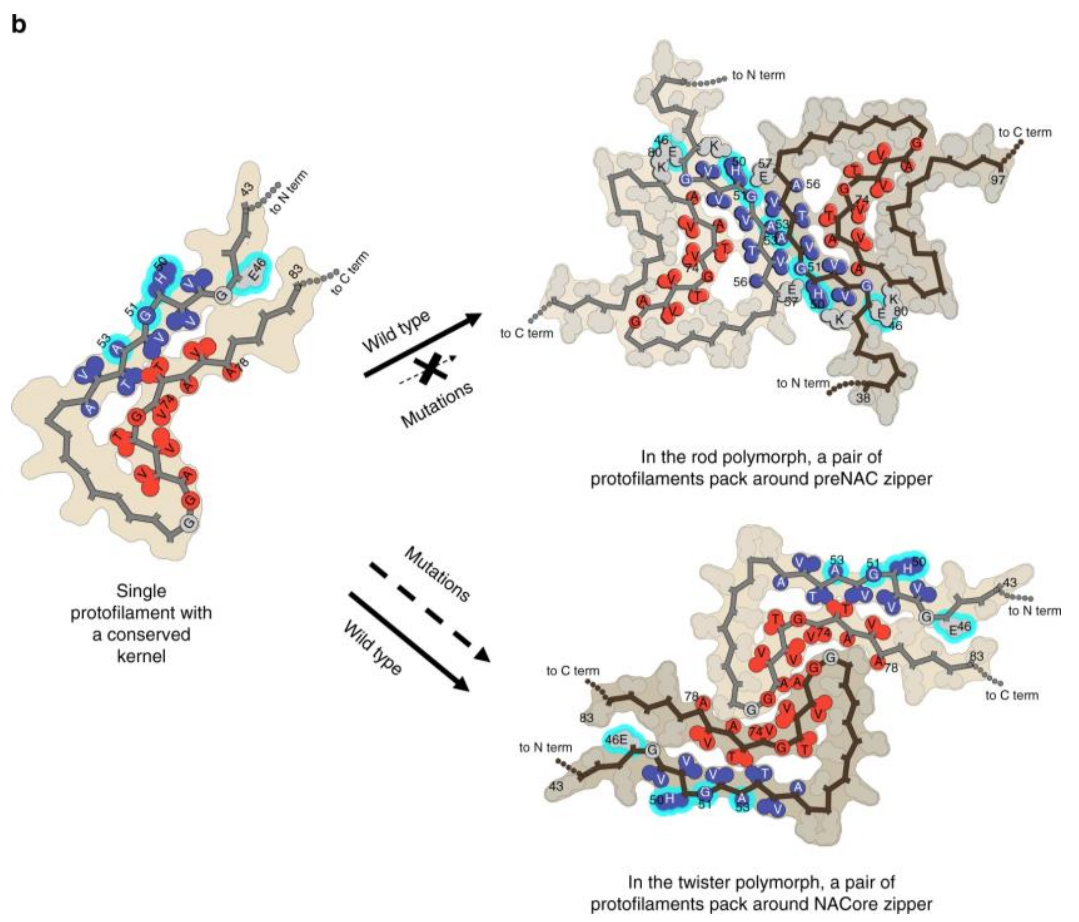

Figure 4.2.1: Comparison of polymorph 1a (rod-polymorph, top) and polymorph $1 \mathrm{~b}$ (twisterpolymorph, bottom) revealing a conserved structural kernel (left).

The relationship of the fibrils in this study to polymorph 2 is more akin to that of $1 \mathrm{a}$ to $1 \mathrm{~b}$ since the monomer folds are only partly similar. A reasonable nomenclature could hence be polymorph 2c. As was outlined earlier, NMR spectroscopy is incapable of discerning single or 
paired filaments, however EM data suggests a double stranded structure for the most prevalent fibrils. A conclusion about the filament interface is hence not possible. Unfortunately, Guerreiro-Ferreira et al. do not report on the morphology of their fibrils, impeding a macroscopic comparison.

Besides the two main motifs of polymorph 1 and 2 characterized by cryo-EM, there have been further studies by ssNMR, that suggest even further polymorphs. ${ }^{[164,166,208]}$ The current study is another example, that the conformational space of $\alpha$-synuclein polymorphism is far from exhausted. Discussion of the influence of familial mutations has so far focused mainly on the inter-filament interactions. ${ }^{[196]}$ Yet, the driving factors behind the formation of the structural elements in the cross-sectional fold remain elusive. It has been argued, that polyanions like phosphate and azide and chaotropes like bromide drive the formation of polymorph 1. ${ }^{[100]}$ This assumption is however contradicted by a study by Barclay et al., who were able to produce the latter structure in the absence of all of these components. ${ }^{[165]}$

In 2012 Comellas et al. argued that phospholipids have only limited influence on the fibril core, reporting minor changes in the $\mathrm{N}$-terminal domain of fibrils with lipids. ${ }^{[91]}$ Upon identifying the greek-key motif in a later study, the group implied that this motif is also found in the presence of lipid bilayers, albeit without giving a direct comparison. ${ }^{[209]}$ Since fibrils were not grown under lipid-free conditions in the course of this study, the absence of an influence on fibril structure cannot be excluded.

The observation of strong lipid-binding by fibrils is in line with previous studies. ${ }^{[210]}$ This behavior is probably due to helical N-termini along the fibril exterior, as indicated by ssNMR (Figure 3.2.9). This distinct membrane binding domain explains, why fibrils exhibit the potential to permeabilize membranes and might account for a certain amount of their toxicity. ${ }^{[84]}$ Accordingly, Comellas et al found minor structural perturbations of the N-terminal resonances in their structures, along with the same cross-peak for V15 and V16. ${ }^{[11]}$ The fact, that the main difference between both preparations is the method of agitation, makes the considerations in chapter 4.1 all the more relevant. 


\subsection{Structure of $\alpha S$ intermediates and insights into nucleation}

To date, the structure and formation of amyloid oligomers and nuclei remains poorly understood. ${ }^{[86]}$ This is to a large extent owed to their transient nature. ${ }^{[15]}$ The protocol introduced here, allowed the enrichment of membrane associated aggregates within hours and their subsequent study in-situ.

The ssNMR spectra of the isolated intermediates I-1 and I-3 feature highly characteristic resonances, allowing conclusions regarding the mechanistic process of fibril formation. The significant amount of structure inside intermediate I-1 suggests, that it is more downstream in the fibril formation process than initial oligomers. This is in line the estimated of $t /$ tlag of $\sim 0.8$, which means it was isolated just before the start of fibril formation. The rapid transition towards I-3 finally initiates nucleation and sets off the fibrillization cascade. The transition from I-1 to $\mathrm{I}-3$ is facilitated because many residues are pre-organized.

The pre-organized residues around T59 and K60 as well as V74 and T75 found in I-1 are located in turns in fibrils(Figure 3.2.5), suggesting the formation of $\beta$-turns as early structural elements during fibrillogenesis. This is corroborated by the sharp increase of anti-parallel $\beta$ sheet content right before fibril formation detected by CD (Figure 4.1.1).

Some of the residues in these putative $\beta$-turns were tentatively assigned by their close proximity of their cross-peaks to fibril peaks. Unlike the T59, K60, V74 and T75, none of these residues exhibits the same chemical shift as in fibril. These chemical shift changes between I-1 and the more fibril-like I-3 suggest that the pre-structured anti-parallel $\beta$-hairpins in $\mathrm{I}-1$ associate and subsequently form parallel $\beta$-sheets characteristic to fibrils.Such a mechanism would require alignment of the $\beta$-turns followed by rotation of the individual strands by $90^{\circ}$ to form cross- $\beta$ sheet hydrogen bonds.

The fact that formation of an anti-parallel hairpin comprising residues V70-K80 is involved in the initial aggregation process, is in line with the finding, that $\Delta 71-82$ mutants of aS show significant reduction in aggregation propensity. ${ }^{[211]} \mathrm{A}$ dodecapeptide of the sequence 71-82 has even been shown to form intermolecular $03 \mathrm{~b} 2-$ sheets $^{[212]}$ and eventually fibrils on its own. ${ }^{[213]}$ The lack of these residues in $\beta$-synuclein in its otherwise predominantly conserved sequence has been linked to its reduced aggregation compared to $\alpha$-synuclein. ${ }^{[214]}$ The sequence $\mathrm{V} 70-\mathrm{K} 80$ is very hydrophobic and inclusion of hydrophobic parts of the protein are a driving force of fibril formation. ${ }^{[15]}$ This yields an additional explanation, why this stretch of residues might start the aggregation process.

Upon comparison of the structures of polymorphs 1 and 2, it is obvious that the formation of turns at V74 and T59 in intermediate structures inherently excludes the formation of 
polymorph 1, where sharp turns are observed at G67 and E83. It is quite remarkable, that Comellas et as found the first structured regions in intermediates to consist of V74-A78 and A90-A91 and therefore residues that form an entirely different architecture in polymorph 1, suggesting a different fibrillization pathway. ${ }^{[91]}$

The role of the interface between to filaments in guiding monomer fold has been discussed at length, especially since the familial mutations H50Q, G51D, A53E and A53T all involve residues directly in the interface. ${ }^{[196]}$ The discovery of polymorph 2 , which seems to readily form for wild-type, does not harbor any steric clashes that would arise from these mutations. Given the incomplete assignments of the fibril spectra at this point, we cannot exclude the influence of the interface in nucleation with certainty.

The apparent tendency of synuclein to form $\beta$-hairpin structures in the presence of phospholipids follows a similar line of argumentation as for oligomerization in chapter 4.1. The binding of $\alpha S$ to membranes is driven by its KTKEGV motif, which inherently forms an amphipathic helix by binding of the Lysine residues to negatively charged lipid head groups (Figure 4.3.1 A). The resulting helix is inserted into packing defects in the membrane. ${ }^{[215]}$ The KTKEGV motif is however disturbed by the sequence G68-A78 (Figure 4.3.1 B, repeat 6) and V74 is oriented towards the lipid core in the helical state (Figure 4.3.1 A, wheel on the right). ${ }^{[196]}$ The membrane disruption by sonication hence facilitates the membrane penetration by V70-K80, by increasing the accessibility of the hydrophobic bilayer core (Figure 4.3.3 C).

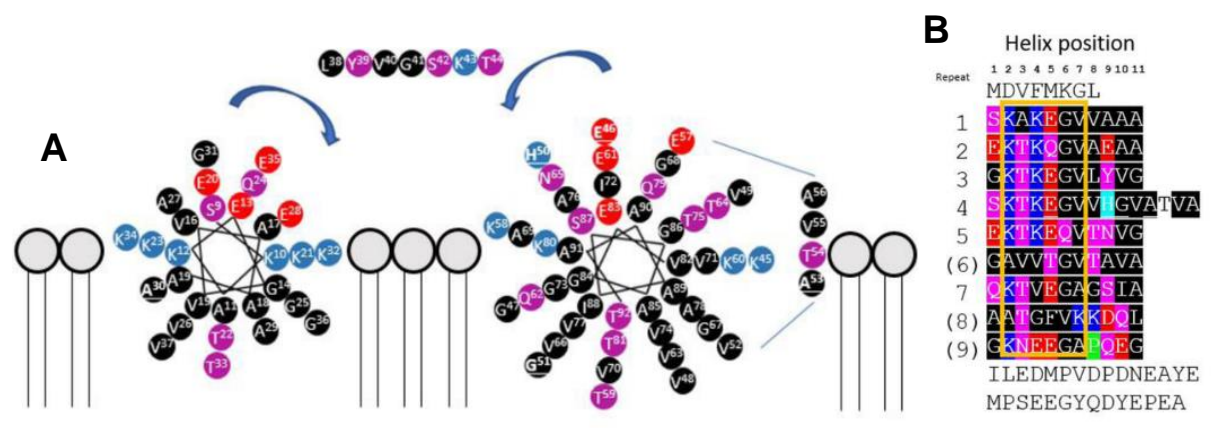

Figure 4.3.1: A) Helical wheel of the membrane associated amphipathic helix (left wheel, residues 1-37, right wheel: residues 45-92); B) Arrangement of aS into KTKEGV imperfect repeats. ${ }^{[196]}$

The insertion of parts of the aS sequence into phospholipid bilayers has been proposed before. A study of Tsigelny et al. regarding the penetration of bilayers by aS by MD simulations, residue N65 was found to be the driving force in spanning a POPC bilayer. ${ }^{[216]}$ Hydrogen bonding of $\mathrm{N} 65$ with water molecules on the other side of the bilayer stabilized a turn encompassing residues 59-72. In polymorph 1 N65 is located in a turn, making it a potential nucleation mechanism. In the center of another turn, residue Q83 offers a sidechain 
potentially capable of a similar mechanism. The gentler agitation methods used by Comellas et al. might hence favor this mechanism, generating polymorph 1. ${ }^{[91]}$
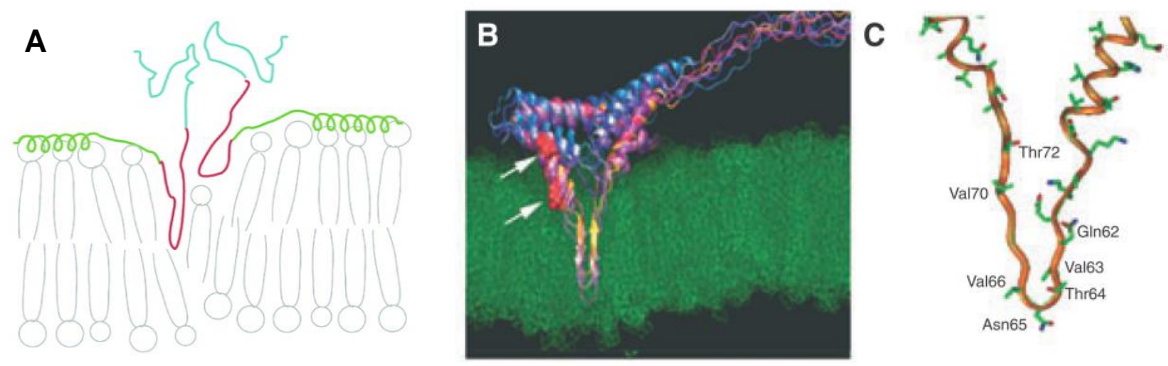

Figure 4.3.2: Insertion of aS into phospholipid bilayers. A) Model by Comellas et a. based on spin diffusion ssNMR. ${ }^{[91]}$ B) MD-simulations carried out by Tsigelny et al., showing the penetration of membranes guided by N65.[216]

Fusco et. al found by paramagnetic tagging of phospholipid tails, that in aS oligomers prepared by lyophilization and subsequent SEC, residues 70-88 experienced paramagnetic relaxation enhancement upon binding to membranes. ${ }^{[00]}$ The authors interpreted this to be the cause of an insertion of a rigid $\beta$-sheet core into the membrane. According to DARR spectra of these oligomers, the structured region is limited to theses residues. Simultaneously residues $10-24$ of the $\mathrm{N}$ terminal domain of the protein adopt an a-helical conformation, when the aggregate is added to phospholipids. Their observations are in surprisingly good agreement with our results, despite the more artificial nature of the oligomer preparation.

Another potential origin for loop formation might be kinks in the membrane bound monomer conformation (Figure 4.3.3 B). EPR studies suggested either a broken "horse-shoe" conformation, containing two anti-parallel helices, ${ }^{[116]}$ as well as a single extended helix. ${ }^{[117]}$ The hypothetic position of the kink between residues V37-T44 was derived from surfactant micelle bound $\alpha S{ }^{[184]} \mathrm{A}$ recent study found a putative kink starting at $\mathrm{K} 60$ for aS monomers binding to nanodiscs. ${ }^{[118]}$ The differences in the observed breaking points of the helix are presumably dependent on the size of the binding partner, as in both cases aS spans the full diameter of the associated particles. Although the broken helix conformation is controversial ${ }^{[117]}$, membrane bound aS might switch between antiparallel and extended helices (Figure 4.3.3 A). Singh et al. used FTIR on artificial peptide helix bundles to show that anti-parallel $\beta$-sheet might form by conversion of neighboring $\alpha$-helices via formation of $3_{10}$-helices. ${ }^{[217]}$ The observed $\beta$-hairpin at 59 could hence originate from an already preformed turn between two anti-parallel helices, which convert into anti-parallel $\beta$-sheets via the aforementioned mechanism. In this case membrane penetration would not be necessary. 


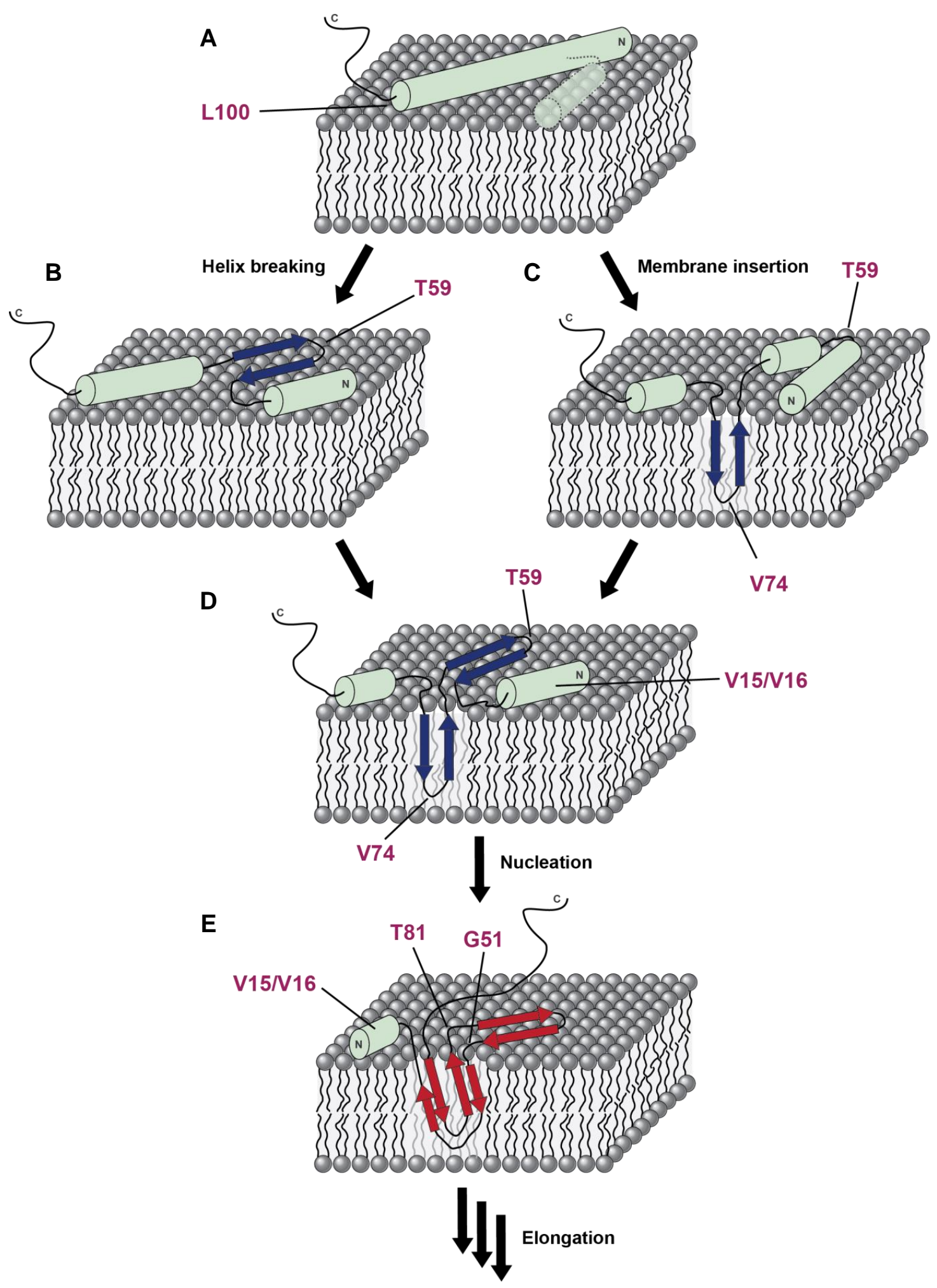

Figure 4.3.3: Schematic model of membrane associated aS aggregation showing two potential pathways of $\beta$-hairpin formation. Folding of a single monomer in an oligomer assembly is shown for simplicity. A) Helical conformation of $\alpha \mathrm{S}$ monomer on membrane and potential switching between anti-parallel and extended helix ${ }^{[18]}$; B) Hairpin formation from anti-parallel helix on membrane (Left) or insertion into membrane (Right), C) Schematic representation of intermediate I-1, comprising to characteristic loops at T59 and V74, as well as a membrane anchoring $\mathrm{N}$-terminal domain (centered at V15/V16). D) Schematic representation of intermediate I-3, formation of a nucleus with a central fibril-like fold, N-terminal domain retains helical conformation, C-terminal domain from T81 on still not with fibril conformation, species subsequently elongates to fibril. 
A

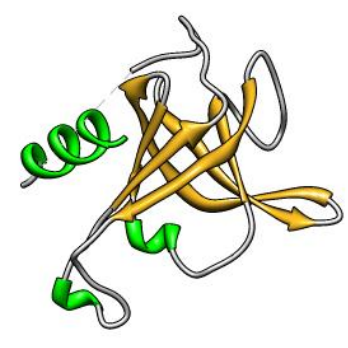

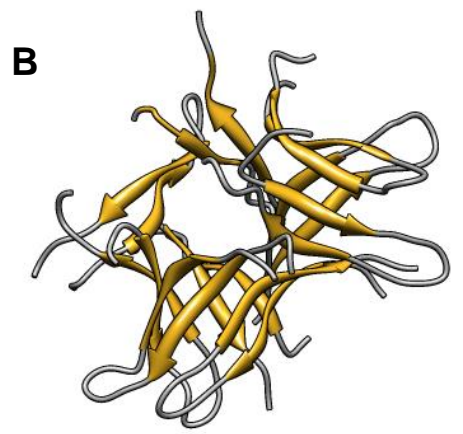

Figure 4.3.4: $\quad$ A) Thrombin Subunit H (CATH code 2.40.10) B) Crystallographically based model of the $\alpha-S_{3} n_{33-58}$ nonamer, showing a basket-shaped structure formed by antiparallel $\beta$-sheets ${ }^{[183]}$

Besides secondary structure calculation from the CD-spectra, BeStSel offers fold recognition. In this approach, the secondary structure content is compared to a database of CATH (Class, Architecture, Topology, Homology) fold categories. Fold recognition of the secondary structure of samples taken at the same time as for the isolation of I-1 (Figure 3.1.10), finds the CATH class 2.40.10 as the most probable fold (Figure 4.3.4 A). This fold carries $\beta$-barrel architecture and the topology of Thrombin Subunit $\mathrm{H}$. Although this fold is highly speculative it carries some resemblance to previously crystallized structures of nonamers from $\alpha \mathrm{S}_{33-58}$ (Figure 4.3.4 B). ${ }^{[183]}$ The sequence of the latter peptide was chosen from micelle bound aS monomers for aforementioned reasons. Based on our results it is possible that such structures are formed by the sequences ${ }_{55}$ VAEKTKEQVTN $_{65}$ and ${ }_{70}$ VVGTVTAVAQK $_{80}$, rationalizing the highly controversial amyloid pores ${ }^{[94]}$ Nevertheless, these results need to be treated with utmost care, as CD spectra were recorded on unpurified samples and further due to experimental limitations. Lastly, these structures are formed by proteins much smaller than aS oligomers, which are usually of the size of $10-30$ monomers. ${ }^{[189,218,219]}$ 


\subsection{Anle138b and its influence on aS aggregates}

Several potential influences of anl138b on protein aggregation have been studied in the course of this thesis. Despite a minor reduction of fibril formation by anle138b, when averaging over several assays, the obtained values are within the margin of error and susceptible to the fluctuations common to ThT fluorescence assays. Nevertheless, the successful decrease of aggregate formation by a [BSA-anle138b] complex, suggests that in order to exert an inhibitory function, anle138b might need a mediator which in the cellular environment might be cytosolic hydrophobic proteins. This finding might explain the disparity between the reduced plaque load found in several PD mouse models and the in vitro studies on anle138b in vesicles presented here. ${ }^{[134,220]}$ Since the strong simplification in the present assays abolishes fibril inhibition, studies on more complex systems, such as neuronal cell cultures might prove more insightful. ${ }^{[221]}$ Nevertheless, since all aggregate forms especially oligomers interact strongly with membranes the chosen systems are relevant for the membrane embedded oligomers. Since serum albumins are not found in the brain, more suitable transporters could be identified to set up in vitro assays that more efficiently simulate a neuronal cell environment. In the light of recent failures inhibitors in phase III clinical studies, it is doubtful, that stopping protein aggregation at the level of fibrils is a suitable strategy in disease prevention. ${ }^{[65]}$ The concept of fibrils being a non-toxic end product of a toxic process, supports this idea. It has even been proposed, that fibrils might act as reservoirs for membrane binding species, such as oligomers. ${ }^{[222]}$

The structural influence of anle138b on fibril formation as detected by ${ }^{13} \mathrm{C}-{ }^{13} \mathrm{C}$-DARR experiments is limited to the C-terminal end of the structured fibril core, comprising perturbations in residues T81, S87 and 188. The studies on intermediates indicate, that this region is among the last to fold into the fibril structure. It is not clear at this point, as to which influence of these structural changes in the fibrils will have, regarding toxicity. Several resonances, that are influenced by the presence of anle138b during fibril growth, could not yet be assigned, so that a completed assignment will help in understanding the influence of the compound.

While the correlations between anle138b and fibrils, observed in DNP-enhanced hNHHC spectra indicate several residue types, the comparison to photoaffinity and MD-simulation results helps in narrowing down the potential binding partners (Figure 4.4.1). MS analysis of the protein in the photoaffinity studies revealed several modified Lysine residues as well as a modified H50 (Figure 4.4.1, green). The MD-simulations, however, indicate binding sites around the aromatic residues Y39 and F94 as well as the aliphatic residue L38 which are all in accordance with the correlation seen at short mixing times in the hNHHC-spectra due to 
their characteristic $C \beta$-shift. The modified residues found by MS are all in very close proximity to these residues, with the exception of $\mathrm{H} 50$. The distance of these labelled sites to the true binding site might be explained by migration of the photoactivated compound, due to stable intermediate states. ${ }^{[180]}$

The observed interaction of anle138b seems to correlate with hydrophobicity of the residues (Figure 4.4.1 B). The exposure of the hydrophobic core in amyloid aggregates has been linked to their toxicity. ${ }^{[223]}$ The small pockets around L38 and Y39 as well as F94 and V95 are among the few residual exposed hydrophobic surfaces of fibril structures. The hydrophobicity of the binding pocket was already predicted by fluorescence spectroscopy studies on anle138b in the presence of $\alpha S \cdot{ }^{[137]} \mathrm{X}$

The published cryo-EM derived structure of polymorph 2 shows residue lle88 enclosed by the $\mathrm{N}$-terminal part of the core (residues 11-20). The absence of cross-linking contacts to $\mathrm{K} 21$ and $\mathrm{K} 23$ as well as K80, are in favor of such a conformation, as the cryo-EM model shows all of these residues facing the inside. The lack of a contact to $\mathrm{K} 80$ also supports the idea, that anle138b does not permanently bind to the sequence around T81, which is structurally altered in the fibril, but rather bind while the structure is in the process of its formation. It was not possible to measure spectra of ${ }^{13} \mathrm{C}$ - or ${ }^{15} \mathrm{~N}$-labelled anle138b at RT, suggesting a more dynamic behavior. The auto-fluorescence of the compound in the presence of fibrils in the absence of lipids, however, is a direct result of the rigidity of its aromatic rings. ${ }^{[137]}$ This seemingly conflicting observation might be explained by the fact, that in NMR experiments in the presence of lipids, large amounts of anle138b partition into the more dynamic lipid environment. In water, anle138b is much more unlikely to unbind due to its hydrophobicity, as was observed in MD-simulations and in fluorescence spectroscopy ${ }^{[137}$, ${ }^{138]}$, while in the presence of phospholipids unbinding is much more probable.
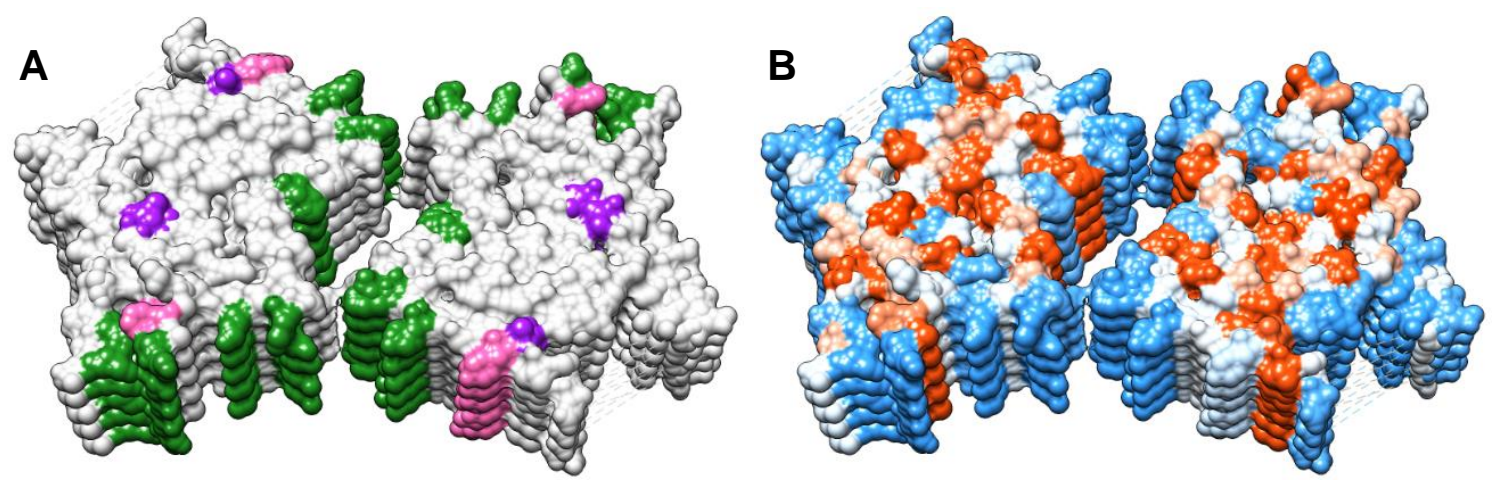

Figure 4.4.1: Solvent excluded surfaces of polymorph 2; PDB models $6 r t 0$ and $6 r t 0$ were interlaced to include as many structured residues as possible; A) Contact map including results from DNP-enhanced NMR (pink: Y39, F94; purple: L38, L88) and photoaffinity studies (green), B) hydrophobicity surface (orange: hydrophobic residues; blue: polar residues). 
Both MD-simulations as well as DNP-enhanced ssNMR experiments support the concept, that anle138b binds to more flexible regions in the aS sequence. Anle138b does not seem to influence the fold of the fibril core itself, so that it must have another mechanism regarding toxicity. The influence on the final fold of the structure, is limited to the C-terminal part of the fibril core, which forms at a later stage. The initial stage of aggregation, hence does not seem to be influence, but potential toxic species arising from initial aggregation might be modulated by anle138b.

In this regard, the influence of the presence of anle138b on the chemical shift of T81 is particularly intriguing (Figure 4.4.2). This residue is located in a turn at the transition to the C-terminal region in fibrils, as determined by TALOS+. This region undergoes structural rearrangement from intermediates towards fibrils. As outlined previously, one of the initial structural elements forming during aS fibrillization is the sequence V70-K80, which might insert into the membrane. The preferred conformation of anle138b in phospholipids, with the bromine facing the inside of the bilayer and the nitrogen atoms forming hydrogen bonds with the solvents (Figure 1.6.2), makes it predisposed for binding to the protein. This way, anle138b binds to the edge of the membrane inserted part of oligomers at T81, stabilizing the membrane. Loop formation by membrane insertion does not seem to be influenced but binding to the adjacent residues might prevent further proliferation of the inserted structure and reduce its membrane disrupting effect. Other residues, that might be influence in this way are $\mathrm{K} 96$ or $\mathrm{K} 97$, as indicated from DNP-enhanced hNHHC-experiments. Once fibril formation starts, the C-terminal region bound by anle138b adopts a different conformation. Folding is thus influenced by binding of the compound to the protein backbone leading to a different arrangement in anle138b-treated fibrils and membrane insertion is reduced, leading to the observation that membranes are less conductive in the presence of fibrils.

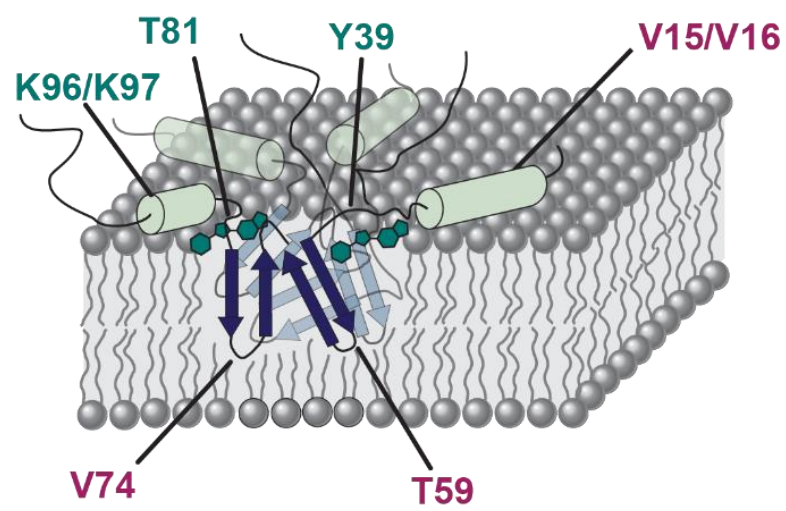

Figure 4.4.2: Putative influence of anle138b on oligomer insertion into phospholipid membranes. The compound binds to the edges of membrane inserted structures due to its already preorganized orientation at the lipid-water interface, reducing the depth of insertion, protecting the membrane from disruption. The initially formed structural elements and hence the core fold are not influence, but the C-terminal domain, which forms much later, is bound and hence perturbed in the fibril. 
Crosslinking experiments hint to an interaction with $\mathrm{N}$-terminal residues (Table 3.3.1). A potential interaction site in oligomers, assuming similarity in binding sites to fibrils, might be located at Y39 as identified by MD-simulations (Figure 3.3.16).

Such a behavior might explain, why amyloid pore induced membrane conductance is observed to a much lesser extent in the presence of anle138b. ${ }^{[224]}$ The binding to aS oligomers in such a manner might prevent full insertion of the toxic species, by stabilizing the edges of the damaged membrane. In the master thesis of Simon Kohlmann, carried out under my supervision, aS oligomers on SLBs on a mica support were studied by AFM (see Figure 3.1.6 for comparison). Oligomers in the presence of membranes containing anle138b displayed an increased averaged height of $1.1 \pm 0.4 \mathrm{~nm}$, compared to an average height of $0.85 \pm 0.2 \mathrm{~nm}$ for control samples. The increase in height was accompanied by an increased width of $9.3 \pm 3.4 \mathrm{~nm}$, compared to $7.6 \pm 2.6 \mathrm{~nm}$ for controls. This result further supports the hypothesis, that insertion of toxic oligomers into lipid membranes is hampered by anle138b.

Following this argumentation anle138b could act as a safeguard during the aggregation process, keeping intermediates from exerting membrane related toxicity and chaperoning the aggregation process until $\alpha S$ in converted into more benign species. 


\section{Conclusions and Outlook}

A key finding of this study is the strong enrichment of aS oligomers under PMCA conditions in the presence of phospholipids. The protocol based is easy to use and yields aggregates in mere hours. Due to this methodology it was possible to prepare intermediate aS species in high enough quantities to study them by ssNMR.

Fibrils prepared under the same conditions displayed extended parallel $\beta$-sheets, containing turns at residues T59, V74 and T81. Comparison to published fibril structure reveals a new polymorph, which is best described as polymorph 2c, due to its structural relationship to polymorph 2, published by Guerrero-Ferreira et al... ${ }^{[100]}$ Fibrils display strong binding to phospholipids, which is attributed to partial conservation of a helical N-terminus, which binds analogous to monomers.

aS oligomers were found to be of $10-20 \mathrm{~nm}$ in size with a globular shape, containing antiparallel $\beta$-sheet and are hence comparable to previously identified oligomers. ${ }^{[92,160]}$ Isolation of on-pathway intermediates was confirmed by ssNMR, displaying distinct ${ }^{13} \mathrm{C}-{ }^{13} \mathrm{C}$ correlation spectra. Comparison of spectra from intermediates and fibrils revealed, that residues T59 and V74 adopt the same chemical shifts as in the fibril. Their location in turns in the fibril structure suggests the conclusion, that the anti-parallel $\beta$-sheet content determined from $C D$, results from formation of $\beta$-hairpins in oligomeric intermediates including the aforementioned residues in the loops. The nature of this folding is guided by the unique combination of PMCA and phospholipids. Sonication disrupts the phospholipid bilayers allowing the insertion of hydrophobic parts of aS in quantitative amounts. In the membrane bound helical aS monomer, both T59 and V74 are oriented towards the core of the bilayer, making them predisposed for membrane penetration.

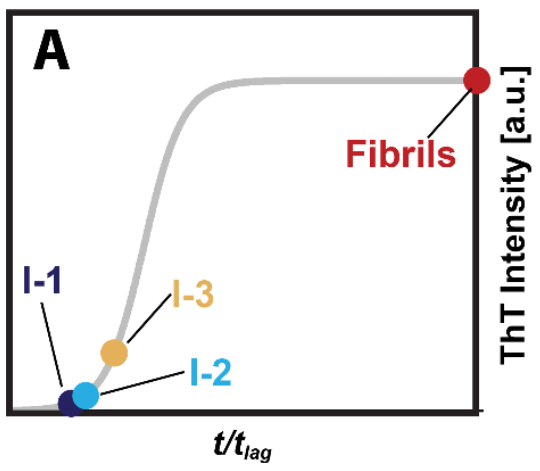

B

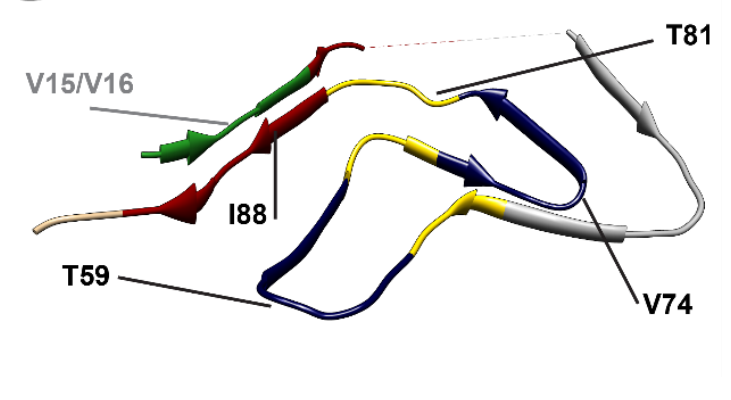

Figure 4.4.1: A) Chronology of the formation of the species based on the ThT values observed at the time of their preparation. B) Development of the fibril structure derived form ${ }^{13} \mathrm{C}-{ }^{13} \mathrm{C}$ DARR spectra of intermediates in fibrils; color coding projected onto PDB entry 6RT0 (polymorph 2)[100]. Initial structured regions are centered around the loops at T59, K60 as well as V74 and T75 in intermediate I-1 (dark blue). Intermediate I-3, isolated at a later stage shows fibril-like structure for residues G51-K80 (yellow). Residues T81-F94 obtain their final structure at a later stage, towards full final formation (red). ain of the protein obtains decreasing helical content through the process of aggregation (green). 
Investigation of intermediates at later stages of the aggregates by ssNMR revealed a more fibril like structure. The formation of oligomers with distinct structural features, which then restructure to form fibrils most probably by converting anti-parallel $\beta$-strands into parallel ones by $90^{\circ}$ rotation is in agreement with a nucleated conformational conversion mechanism. ${ }^{[204]}$ Further insight is expected, since due to time restrictions not all spectra could be evaluated to the full extent. The quality of those spectra however is good enough to expect further assignments to be obtained without further measurement. The use of ${ }^{13} \mathrm{C}-{ }^{13} \mathrm{C}$ PAR NMR might help in final determination of the folding of the aS monomer within the cross-section of the fibrils, as has been demonstrated previously. ${ }^{[100]}$

The transient and on-pathway nature of $\alpha S$ oligomers makes it all the more remarkable, that ssNMR studies at RT revealed high peak intensities and reasonable stability. These results give confidence, that sequence specific assignments, as it has been done for fibrils will also be feasible for oligomers. Through space correlation experiments, such as ${ }^{13} \mathrm{C}-{ }^{15} \mathrm{~N}$-TEDOR might give novel insight into the structure of the so far poorly characterized species.

Further mechanistic insights into fibril folding can be gained from oligomers prepared at even earlier $t / t_{\text {lag }}$ times, as the intermediates presented in this work are very close to and even past nucleation. Functional studies, such as BLM or cell viability assays, need to be performed to allow conclusions about their disease related properties and toxicity. The identification of structural elements in oligomers has a direct application in guiding MD simulations, which have often been based on monomer and fibril states. ${ }^{[96,225]}$ Although oligomer formation of oligomers containing $\beta$-hairpins has been studied before, the location of the loop has usually been derived from the structure of micelle bound monomer. ${ }^{[183,226]}$

Two binding sites for aS on fibrils could be identified in Y39 and F94, located at the edges of the structured core independent of polymorph structure. Further it was revealed that anle138b favors dynamic hydrophobic domains, in accordance with previously published results. ${ }^{[139]}$ At this point it is unclear, which effect this binding has on disease progression. Further studies are needed to clarify the influence of anle138b on fibril formation. The successful reduction of aggregate formation without a change in lag time in the presence of BSA suggests, that anle138b might have a thermodynamic, rather than a kinetic effect. This might explain the observation, that aS aggregates are reduced in their density in PD mouse models. ${ }^{[134]}$ The fibrils might be destabilized or modulated in such a way, that their assembly is reduced.

The interaction of anle138b with aS oligomers was identified by DNP-enhanced ssNMR, however incomplete assignments impaired the exact identification of a binding site but the residue type responsible for binding could be narrowed down to Lysine or Glutamate. The 
assignment of the cross-peaks of the interacting residues has not been achieved, yet, due to limited time. Completion of the assignments will unravel the binding site of the compound a posteriori. Improvement of crosslinking derivatives regarding their selectivity might also assist in the localization of interacting residues.

The structural perturbations in aS fibrils caused by anle138b are minor and concern mostly the C-terminal part of the fibrils, which forms at a later stage in the aggregation. Membrane inserted domains of aS aggregates involved in initial aggregation are not influenced with respect to the final fold, however the insertion into membranes might be impeded. This is suggested due to the chemical shift effect observed on T81, as determined by TALOS+ in the presence versus absence of anle138b. T81 is located in a turn region in the fibrils and at the end of the membrane inserting stretch $\mathrm{V} 70-\mathrm{K} 80$, and right at the transition to the Cterminal part of the fibril. T81 undergoes structural rearrangement when restructuring from oligomers towards fibrils occurs. The preferred conformation of anle138b in phospholipids, with the bromine facing the inside of the bilayer and the nitrogen atoms forming hydrogen bonds with the solvents, makes it predisposed for binding to those residues of the protein which insert in the membrane. In this way, anle138b might bind to the edge of the membrane inserted part of oligomers, stabilizing the membrane. Loop formation by membrane insertion does not seem to be influenced but binding to the adjacent residues might prevent further proliferation of the inserted structure and reduce its membrane disrupting effect.

The model of reduced insertion is supported by the observed reduction in amyloid pore insertion into phospholipid membranes, leading to reduced conductivity. ${ }^{[224]}$ Further, in a master thesis under my supervision, Simon Kohlmann found by AFM, that aS oligomers in the presence bilayers containing anle138b show increased height and width compared to controls, Indicating that aggregates had not fully penetrated the membrane. Although anle138b does not fully inhibit protein aggregation, it seems to lessen the toxic effects of the species, most probably oligomers, involved in the process and reduces aggregate load. This is in agreement in with the improved disease related symptoms and reduced amyloid load in mouse models of PD, as well as MSA. ${ }^{[132,134,220]}$

This thesis sheds light on the mechanism underlying fibril formation. Intermediates of aS were successfully isolated and characterized, revealing structural elements of early aggregates. Anle138b interferes with this process, influencing both fibril fold and amount. Although the exact disease modifying mechanism is yet to be revealed, binding sites for anle138b have been identified in both fibrils and oligomers. These results lay the basis for a more wholesome understanding of protein aggregation and its modulation in disease. 


\section{Abbreviations}

aS

- a-synuclein

$A \beta$

- Amyloid- $\beta$

AD

- Alhzeimer's disease

AFM

- Atomic Force Microscopy

ALS - amyotrophic lateral sclerosis

BLM - Black lipid membrane

BSA - Bovine Serum Albumin

CD - circular dichroism

CNS - central nervous system

COSY - Correlation Spectroscopy

DBS - deep brain stimulation

DLS - dynamic light scattering

DMPS - 1,2-dimyristoyl-sn-glycero-3-phospho-L-serine

DMSO - dimethyl sulfoxide

DSS - 4,4-dimethyl-4-silapentane-1-sulfonic acid

HMBC - Heteronuclear Multiple Bond Correlation

HR-MAS - High Resolution Magic Angle Spinning

HSQC - Heteronuclear Single Quantum Coherence

IDP $\quad$ - intrinsically disordered protein

L/P - lipid to protein ratio

MS - multiple sclerosis

NAC - non-amyloid-beta-component

NDD - neurodegenerative disease

NMR - Nuclear Magnetic Resonance

NOE - Nuclear Overhauser effect

NOESY - Nuclear Overhauser effect Spectroscopy

MS - Mass Spectrometry

MSA - Multiple System Atrophy

PD - Parkinson's Disease

PMCA - Protein Misfolding Cyclic Amplification

POPA - 1-palmitoyl-2-oleoyl-sn-glycero-3-phosphate

POPC - 1-palmitoyl-2-oleoyl-sn-glycero-3-phosphocholine 
DOPE - 1,2-dioleoyl-sn-glycero-3-phosphoethanolamine

DOPS - 1,2-dioleoyl-sn-glycero-3-phospho-L-serine

RT

- room temperature

SUV

- Small Unilamellar Vesicle

LUV

- Large Unilamellar vesicle

GUV

- Giant Unilamellar Vesicle

TEM

- transmission electron microscopy

ThT

- Thioflavin T 


\section{Materials and Methods}

\subsection{Instrumentation}

\begin{tabular}{|c|c|}
\hline Common Name & Identifier/Company \\
\hline$-80^{\circ} \mathrm{C}$ freezer & MDF-U71V Ultra-low temperature freezer, SANYO Electric \\
\hline Balances & A 200 S - Sartorius Göttingen, Germany \\
\hline Centrifuges & 54150 Eppendorf Hamburg, Germany \\
\hline DLS & Corporation DynaPro DLS Wyatt Technology Dernbach, Germany \\
\hline $\begin{array}{l}\text { Fluorescence } \\
\text { Spectroscopy }\end{array}$ & Cary Eclipse - Varian \\
\hline CD Spectroscopy & J-815-150S, Jasco Deutschland GmbH, Pfungstadt, Germany \\
\hline Lyophylisation & Biotech Christ Alpha 2-4 - B. Braun Melsungen, Germany \\
\hline NMR spectrometers & $\begin{array}{l}700 \mathrm{MHz} \text { Avance III HD with CP-TCI HCND probe with z-gradient } \\
900 \mathrm{MHz} \text { Avance I with CP-TCI HCND probe with z-gradient } \\
\text { all Bruker Karlsruhe, Germany }\end{array}$ \\
\hline NMR tubes & $\begin{array}{l}177.8 \text { × } 3 \text { mm NMR tubes typ standard - Hilgenberg Malsfeld, } \\
\text { Germany } \\
178 \times 5 \text { mm NMR tubes, Hilgenberg Malsfeld, Germany }\end{array}$ \\
\hline ssNMR spectrometers & $\begin{array}{l}800 \mathrm{MHz} \text { Avance III HD with } 1.3 \mathrm{~mm} \text { HRMAS HCN probe } \\
850 \mathrm{MHz} \text { Avance III with } 3.2 \mathrm{~mm} \text { HRMAS HCN probe } \\
\text { all Bruker Karlsruhe, Germany }\end{array}$ \\
\hline $\begin{array}{l}\text { DNP-enhanced } \\
\text { ssNMR }\end{array}$ & $\begin{array}{l}600 \mathrm{MHz} \text { Avance III with } 3.2 \mathrm{~mm} \mathrm{H} / \mathrm{C} / \mathrm{N} \text { DNP LT MAS probe and } 7.2 \\
\text { T cryogen free gyrotron } \\
\text { all Bruker Karlsruhe, Germany }\end{array}$ \\
\hline ssNMR rotors & $\begin{array}{l}\text { DNP: } \mathrm{HZ16916} \mathrm{BL3.2} \\
\text { PH MAS roto } \mathrm{ZrO}_{2} \\
\text { Both Bruker Karlsruhe, Germany }\end{array}$ \\
\hline pH-meter & PB-11 (Sartorius Göttingen, Germany) \\
\hline Sonicaton & $\begin{array}{l}\text { RK } 103 \text { H (Bandelin Sonorex Berlin, Germany) } \\
\text { Q700 (Qsonica Newtown CT, USA) }\end{array}$ \\
\hline Incubators & $\begin{array}{l}\text { Innova } 4000 \text { (New Brunswick Scientific, Edison NJ, USA) } \\
\text { Heratherm General Protocol (Thermo Fisher, Waltham MS, USA) }\end{array}$ \\
\hline AFM & Nanowizard 4 (JPK BioAFM/ Bruker Nano GmbH, Berin, Germany) \\
\hline TEM & $\begin{array}{l}\text { Phillips CM120 with TemCam } 224 \text { A slow scan camera (TVIPS, } \\
\text { Germany) }\end{array}$ \\
\hline
\end{tabular}

Table 7.1.1: List of instruments used in this work. 


\subsection{Software}

\begin{tabular}{|l|l|}
\hline Program & Reference \\
\hline Avogadro 1.0.3 & http://avogadro.cc/wiki/Main_Page \\
\hline CcpNmr Analysis 2.2 & http://www.ccpn.ac.uk/ \\
\hline TopSpin 3 pl. 3.5.7 & Bruker Biospin, Karlsruhe, Germany \\
\hline NMRPipe/NMRDraw & spin.niddk.nih.gov/NMRPipe \\
\hline Sparky 3.114 & www.cgl.ucsf.edu/home/sparky \\
\hline Origin 9.0.0 & OriginLab Corporation, Northampton USA \\
\hline UCSF Chimera & https://www.cgl.ucsf.edu/chimera/ \\
\hline TALOS+ & https://spin.niddk.nih.gov/NMRPipe/talos/ \\
\hline BestSel & http://bestsel.elte.hu/ \\
\hline CSI 3.0 & http://csi3.wishartlab.com/cgi-bin/index.php \\
\hline
\end{tabular}

Table 7.2.1: List of software used in this work.

\subsection{Materials}

All the substances used in this work are listed in Table 7.3.1.

Anle138b as well as ${ }^{15} \mathrm{~N}$ - and ${ }^{13} \mathrm{C}$-labelled anle138b were synthesized and kindly provided by Andrei Leonov and Sergey Ryazanov at the Max Planck Institute for Biophysical Chemistry Göttingen.

The crosslinking derivative krru133 was synthesized and kindly provided by Kris Runge at the Max Planck Institute for Biophysical Chemistry Göttingen.

$\alpha$-synuclein as well as $\mathrm{U}-{ }^{15} \mathrm{~N}, \quad \mathrm{U}-{ }^{13} \mathrm{C}, \quad \mathrm{U}-{ }^{15} \mathrm{~N}-{ }^{13} \mathrm{C}$ and $\mathrm{U}-{ }^{2} \mathrm{H}-{ }^{15} \mathrm{~N}-{ }^{13} \mathrm{C}$ synthesized by Karin Giller and Melanie Wegstroth kindly provided by Stefan Becker at Max Planck Institute for Biophysical Chemistry Göttingen as a $300 \mu \mathrm{M}$ solution in $50 \mathrm{mM}$ HEPES with $100 \mathrm{mM} \mathrm{NaCl}$ at $\mathrm{pH} 7.4$. 


\begin{tabular}{|c|c|}
\hline Substance/Solvent & Supplier \\
\hline $\begin{array}{l}\text { 4,4-Dimethyl-4-silapentane-1-sulfonic acid } \\
\text { (DSS) }\end{array}$ & WILMAD \\
\hline$\alpha$-synuclein & Karin Giller, Melanie Wegstroth \\
\hline anle138b & Andrei Leonov and Sergey Ryazanov \\
\hline krru133 & Kris Runge \\
\hline Chloroform & Merck \\
\hline $\mathrm{D}_{2} \mathrm{O}$ & Euriso-top \\
\hline DMSO & Merck \\
\hline HEPES & Roth \\
\hline Glyin & Merck \\
\hline $\mathrm{NaCl}$ & Merck \\
\hline $\mathrm{MgCl}_{2}{ }^{*} 6 \mathrm{H}_{2} \mathrm{O}$ & Merck \\
\hline POPA & Avanti Polar Lipids \\
\hline POPC & Avanti Polar Lipids \\
\hline$d_{64}-\mathrm{POPC}$ & Avanti Polar Lipids \\
\hline$d_{64}$-POPA & Avanti Polar Lipids \\
\hline DOPE & Avanti Polar Lipids \\
\hline DOPS & Avanti Polar Lipids \\
\hline Thioflavin T & Sigma \\
\hline
\end{tabular}

Table 7.3.1: Reagents used in this work

\subsection{Buffers}

The buffers used in this work are listed in Table 7.4.1.

Buffers were prepared by weighing in the desired amount of HEPES or Glyine, dilution in solvent and adjustment of the $\mathrm{pH}$ by addition of a saturated $\mathrm{NaOH}$ solution.

\begin{tabular}{|c|c|c|c|c|}
\hline No. & Buffer & $\mathrm{pH}$ & Solvent & Application \\
\hline 1 & $50 \mathrm{mM}$ HEPES, $100 \mathrm{mM} \mathrm{NaCl}$ & 7.4 & $\mathrm{H}_{2} \mathrm{O}$ & \\
\hline 2 & $50 \mathrm{mM}$ HEPES, $100 \mathrm{mM} \mathrm{NaCl}$ & 7.4 & $\mathrm{D}_{2} \mathrm{O}$ & NMR \\
\hline 3 & $10 \mathrm{mM}$ HEPES, $150 \mathrm{mM} \mathrm{NaCl}, 1 \mathrm{mM} \mathrm{MgCl} 2$ & 7.4 & $\mathrm{H}_{2} \mathrm{O}$ & AFM \\
\hline 4 & 5 mM HEPES & 7.4 & $\mathrm{H}_{2} \mathrm{O}$ & $\begin{array}{l}\text { CD-spectroscopy, } \\
\text { ssNMR packing }\end{array}$ \\
\hline 5 & 50 mM Glycine & 8.5 & $\mathrm{H}_{2} \mathrm{O}$ & ThT-fluorescence \\
\hline
\end{tabular}

Table 7.4.1 Buffers used in this work 


\subsection{Methods}

\subsubsection{Curve Fitting}

Curve Fitting was performed in Origin Pro 9.0 (OriginLab Corporation, Northampton, MA, USA)

\subsubsection{Preparation of Small Unilamellar Vesicles (SUVs)}

Phospholipids were obtained from Avanti Polar Lipids and used without further treatment. POPA and POPC were dissolved in $1.2 \mathrm{~mL}$ chloroform and $1 \mathrm{~mL}$ of each solution was added to obtain a molar ratio of $1: 1$ for the lipids. For the preparation of SUVs containing anle138b, the compound was dissolved in chloroform and added to the lipid solution, resulting in a lipid to compound ratio of 20 . The solvent was evaporated under a $\mathrm{N}_{2}$-beam and the sample was lyophilized overnight to remove residual solvent. The resulting lipid film was hydrated with buffer (50 mM HEPES, $100 \mathrm{mM}, \mathrm{pH}$ 7.4). Hydrated samples were sonicated at $37 \mathrm{kHz}$ (four times for 10 min with 15 min breaks or until the sample was translucent) in a glass tube. The resulting solution was and filtered through $0.22 \mu \mathrm{m}$ sterile filters to obtain SUVs with an average hydrodynamic diameter of $48 \pm 3 \mathrm{~nm}$ for vesicles containing anle138b and $41 \pm 2 \mathrm{~nm}$ for vesicles without the compound.

For photoaffinity studies, DOPC and DOPS were dissolved in Chloroform to yield solutions of $3 \mathrm{mM}$ and $3.5 \mathrm{mM}$ respectively. Phospholipid solutions were combined to obtain a molar ratio of 7:3 (DOPC/DOPS). Krru133 was dissolved in chloroform and added to the lipid solution at a molar ratio of 20:1 (lipid/krru133). Vesicles were prepared in an analogous to the procedure mention above.

\subsubsection{ThT Fluorescence Measurements}

For the quantification of amyloid fibrils, Thioflavin T (ThT) is commonly used as a stain in vitro. ${ }^{[227]}$

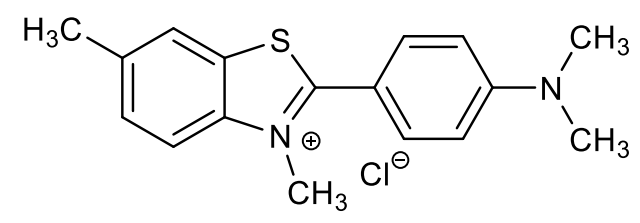

Figure 7.5.1: Chemical Structure of Thioflavin T.[227] 
Thioflavin $T$ is sensitive to the $\beta$-sheet structure in amyloid fibrils and upon binding exhibits a spectral shift of $100 \mathrm{~nm}$ for excitation $\left(\lambda_{\mathrm{ex}}=342 \mathrm{~nm}\right.$ to $442 \mathrm{~nm}$ ) and $50 \mathrm{~nm}$ for $\left(\lambda_{\mathrm{em}}=430 \mathrm{~nm}\right.$ to $482 \mathrm{~nm}$ ). This large fluorescence spectral shift allows selective excitation of amyloid fibrils bound to ThT. A solution of $1 \mathrm{mM}$ ThT in $50 \mathrm{mM}$ Glycine Buffer at pH 8.5 was prepared and stored at $4{ }^{\circ} \mathrm{C}$ under light exclusion until use. Solutions were freshly prepared if older than one week. On the day of use, $0.1 \mathrm{~mL}$ of the $1 \mathrm{mM}$ ThT solution were mixed with $1.9 \mathrm{~mL}$ of buffer to obtain $2 \mathrm{~mL}$ aliquots of working solution. $5 \mu \mathrm{L}$ of the aggregating solution were added to the working solution and fluorescence emission spectra of the resulting solution were measured using a Varian Cary Eclipse fluorescence spectrometer. Experimental parameters are listed in Tab. 5.9. For every spectrum three scans were performed.

$\begin{array}{ll}\text { Slid width: } & 1 \mathrm{~nm} \\ \text { Scan Range: } & 464-600 \mathrm{~nm} \\ \text { Excitation: } & 446 \mathrm{~nm} \\ \text { Excitation-bandwidth: } & 10 \mathrm{~nm} \\ \text { Excitation: } & 446 \mathrm{~nm} \\ \text { Excitation-bandwidth: } & 10 \mathrm{~nm} \\ \text { Integration time: } & 0.1 \mathrm{~s}\end{array}$

Table 7.5.1: Experimental parameters used for fluorescence spectroscopic measurements

\subsubsection{Solution NMR Spectroscopy}

NMR experiments were recorded on Bruker $700 \mathrm{MHz}$ (Avance III HD with CP-TCI HCND probe with z-gradient) and $900 \mathrm{MHz}$ (Avance I with CP-TCI HCND probe with z-gradient) spectrometers. Temperature during measurements was kept at $15^{\circ} \mathrm{C} .{ }^{1} \mathrm{H} 1 \mathrm{D}$ NMR spectra were acquired using presaturation, applying the "zgpr.dl" pulse sequence using 128 scans and a relaxation delay of $2.0 \mathrm{~s} .{ }^{1} \mathrm{H}-{ }^{15} \mathrm{~N}-\mathrm{HSQC}$ spectra were acquired using the hsqc $15 \mathrm{~N} . \mathrm{dl}$ pulse sequence using $2048 \times 256$ scans and a relaxation delay of $1.2 \mathrm{~s}$.

Processing and analysis of 1D data sets was carried out in TopSpin (Bruker). 2D data sets were processed in NMRPipe and analyzed in CcpNmr Analysis. Peaklists were exported and analyzed using a custom written python script. 


\subsubsection{Circular Dichroism Spectroscopy}

Spectra were acquired on a J-815-150S (JASCO), with a single position peltier cell holder (PTC-514) for temperature control. Samples were measured in 110-QX cuvette (HELLMA) with a path length of $1 \mathrm{~mm}$.

$\begin{array}{ll}\text { Measure range: } & 260-180 \mathrm{~nm} \\ \text { Data pitch: } & 1 \mathrm{~nm} \\ \text { D.I.T.: } & 1 \mathrm{sec} \\ \text { Bandwidth: } & 1.00 \mathrm{~nm} \\ \text { Scanning speed: } & 20 \mathrm{~nm} / \mathrm{min} \\ \text { Accumulations: } & 5 \\ \text { Temperature: } & 20{ }^{\circ} \mathrm{C}\end{array}$

Figure 7.5.2: Parameters used for CD measurements.

\subsubsection{Atomic Force Microscopy (AFM)}

Images were acquired on a Nanowizard 4 (JPK) with a Vortis SPMControl station. The instrument was mounted on an halcyonics_i4 vibration isolation unit (Accurion) and encased in a custom-made acoustic enclosure hood. Measurements were carried out using BioLever Mini cantilevers (Olympus, BL-AC40TS-C2) with a spring constant of $0.09 \mathrm{~N} / \mathrm{m}$ and a resonance frequency of $100 \mathrm{kHz}$, mounted on a supercut glass cantilever holder (JPK).

Samples of aS in the presence of phospholipids were incubated on freshly cleaved slabs of mica, fixed on stainless steel metal discs $(d=15 \mathrm{~mm}$ ) by 2 Ton Epoxy (Devcon). After incubation for $20 \mathrm{~min}$, samples were washed with $3 \times 70 \mu \mathrm{L}$ buffer (10 mM HEPES, $150 \mathrm{mM} \mathrm{NaCl}, 1 \mathrm{mM} \mathrm{MgCl}_{2}, \mathrm{pH}$ 7.4).

Height images were acquired in liquid using intermittent mode (AC mode).

\subsubsection{Aggregation Assays}

Monomeric aS was centrifuged for $1 \mathrm{~h}$ at $55.000 \mathrm{rpm}$ at $4{ }^{\circ} \mathrm{C}$ and the supernatant was added to a solution of phospholipid SUVs different L/P ratios and $\mathrm{NaN}_{3}(0.02 \mathrm{w}-\%)$ to obtain a final protein concentration of $70 \mu \mathrm{M}$. If not mentioned otherwise L/P ratio was $10(n / n)$. Buffer concentration was kept at $50 \mathrm{mM}$ HEPES, $100 \mathrm{mM} \mathrm{NaCl}$ at $\mathrm{pH}$ 7.4. For aggregation in the presence of BSA and compounds, BSA was prepared as a saturated solution by vortexing the protein for $2 \mathrm{~min}$ at RT and filtering through a $0.22 \mu \mathrm{m}$ sterile filter. Anle138b was dissolved in DMSO at a concentration of $165 \mathrm{mM}$. $1.5 \mu \mathrm{L}$ of this solution were added to 750 $\mu \mathrm{L}$ of BSA solution and the resulting solution was vortexed for $2 \mathrm{~min}$ at RT. Concentration of 
protein was determined by UV $\left(\varepsilon_{280}=43824 \mathrm{~cm}^{-1} \mathrm{M}^{-1}\right)$ to be appox. $400 \mu \mathrm{M}$, while concentration of anle138b in the complex was estimated to be $600 \mu \mathrm{M}$ $\left(\varepsilon_{270}(\mathrm{DMSO})=25000 \mathrm{~cm}^{-1} \mathrm{M}^{-1}\right)$ Concentration The resulting solution was centrifuged for $4 \mathrm{~min}$ at $16.5 \mathrm{rpm}$ and the supernatant was filtered through a $0.22 \mu \mathrm{m}$ filter. BSA was added to the aggregation sample, at a ratio of $\alpha S / B S A=2: 1$.

PMCA was performed using a Q700 (Q700-110 = sonication device by QSonica, with a Microplate Horn Assembly (431MPX) and a Compact Recirculating Chiller (4900-110). The samples were sonicated for $30 \mathrm{~s}(20 \mathrm{kHz})$ at $37{ }^{\circ} \mathrm{C}$ followed by an incubation period or 30 min. Average power output ranged from 250-300 W.

Orbital Shaking was performed using an Innova 4000 incubator, operated at $37^{\circ} \mathrm{C}$ and $300 \mathrm{rpm}$.

Aggregation under quiescent conditions was carried out by placing samples in a drying cabinet at $37^{\circ} \mathrm{C}$

Samples of the aggregating samples were taken at characteristic time points and analyzed with several methods. The samples and their preparation are listed in Table 7.5.2.

\begin{tabular}{|l|c|c|l|}
\hline Method & $\mathbf{V}_{\text {sample }}[\boldsymbol{\mu L}]$ & $\mathbf{V}_{\text {Buffer }}[\boldsymbol{\mu L}]$ & Buffer composition \\
\hline${ }^{1} \mathrm{H}-{ }^{15} \mathrm{~N}-\mathrm{HSQC}$ & 120 & 20 & $\begin{array}{l}\mathrm{D}_{2} \mathrm{O}, 100 \mu \mathrm{M} \text { DSS, } 50 \mathrm{mM} \text { HEPES, } \\
100 \mathrm{mM} \mathrm{NaCl}, \mathrm{pH} 7.4\end{array}$ \\
\hline CD & 15 & 165 & $5 \mathrm{mM}$ HEPES, pH 7.4 \\
\hline ThT & 5 & 2000 & $50 \mathrm{mM}$ Gly, pH 8.5 \\
\hline AFM & 70 & $\geq 210$ & $\begin{array}{l}10 \mathrm{mM} \text { HEPES, } 150 \mathrm{mM} \mathrm{NaCl}, 1 \mathrm{mM} \mathrm{MgCl}_{2}, \\
\text { pH } 7.4\end{array}$ \\
\hline EM & 20 & - & 5 mM HEPES, pH 7.4 \\
\hline
\end{tabular}

Table 7.5.2: Sample composition for subsequent analysis of the aggregating samples.

\subsubsection{Photoaffinity studies}

Sample preparation was carried out analogous to chapter Error! Reference source not found.. aS was added to a solution of vesicles consisting of DOPC, DOPS and krru (see Error! Reference source not found.) to yield a final protein concentration of $30 \mu \mathrm{M}$ and a $\mathrm{L} / \mathrm{P}$ ratio of 40 . The samples were subjected to PMCA treatment and samples were taken at characteristic time point and immediately stored on ice. Samples were subsequently irradiated by UV-light. After irradiation was finished, the protein was precipitated by Acetone and subjected to Tryptic digestion. The peptide fragments were then analyzed by ESI-MS. 
Tryptic digestion as well as MS-experiments and analysis were carried out in the lab of Prof. Dr. H. Urlaub at MPIBPC, Göttingen.

\subsubsection{Transition Electron Microscopy}

TEM measurements were performed by Gudrun Heim in the workgroup of Dietmar Riedel at the Max Planck Institute for Biophysical Chemistry in Göttingen. Samples were adsorbed onto 400 mesh carbon-coated copper grids and the buffer was removed using a filter paper. Subsequently, samples were stained by the addition of $1 \%$ uranyl acetate solution, which was subsequently dried with a filter paper. If images quality was low or micrographs were too crowded, samples were washed with water and diluted by a factor of 1:50 before measurement. Images were taken at RT in a Philips CM120 electron microscope (Philips Inc.) at a defocus of $2.3 \mathrm{~mm}$ using a TemCam 224 A slow scan CCD camera (TVIPS, Gauting, Germany).

\subsubsection{Estimation of oligomer concentration from kinetic data}
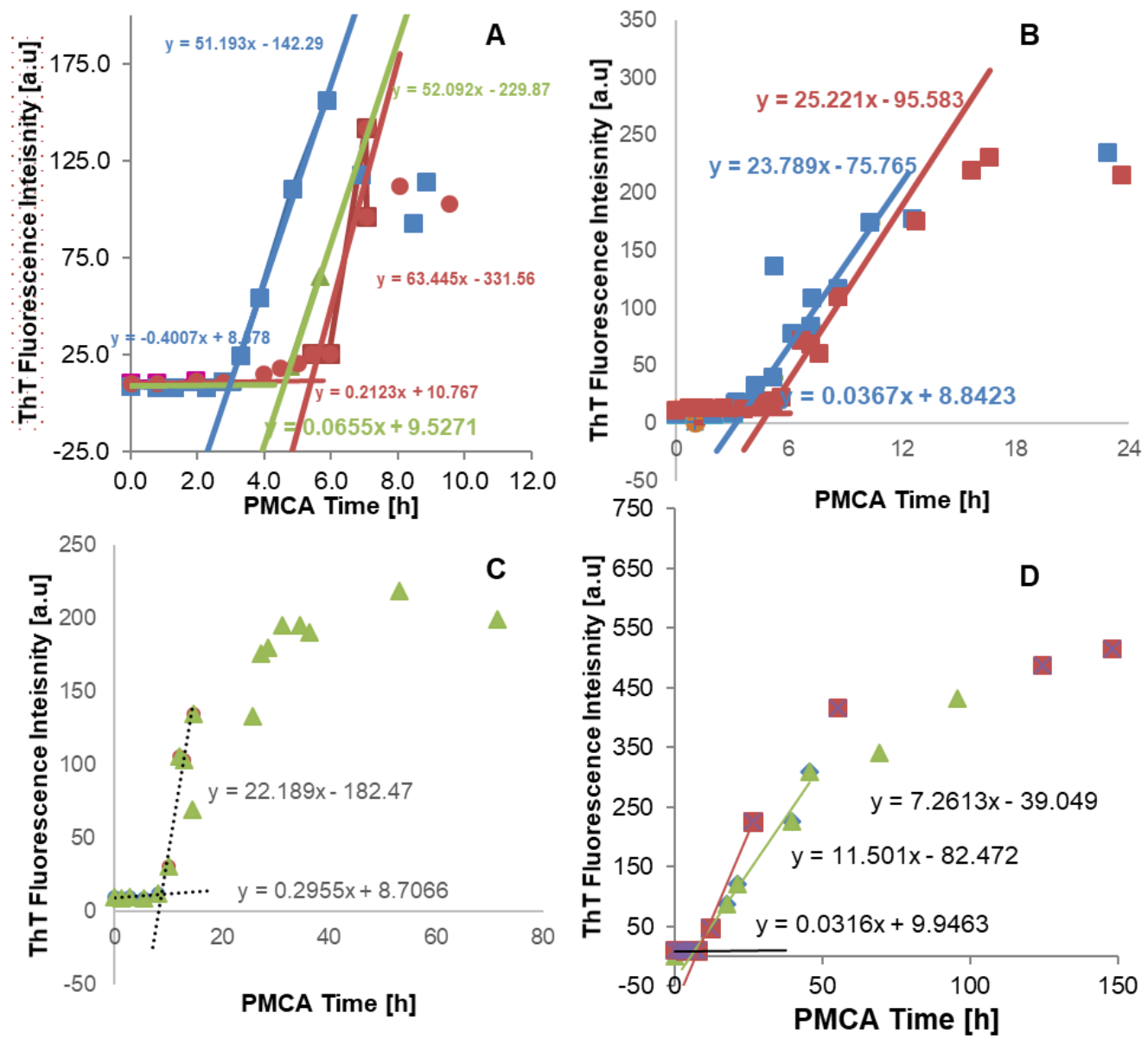

Figure 7.5.3 Linear fitting to experimental data of PMCA in the presence of phospholipids in the absence (A) and presence (B) of anle138b, as well as lipid free (C) and orbital shaking (D) experiments. 
Tangents of the points of maximum fibrilization rate were approximated fitting a linear function points to the steepest part of the Intensity of ThT fluorescence. This involved the first 4-5 datapoints of each series. The initial baseline was approximated by fitting a linear function to the first 4 datapoints of each series. The lag times were of the individual aggregation assays were estimated by equating the resulting functions and solving for $t$, resulting in the equation

$$
t_{\text {lag }}=\frac{c_{2}-c_{1}}{m_{1}-m_{2}}
$$

Where $m_{1}$ and $c_{1}$ are the slope and constant of the tangent fit and $m_{2}$ and $c_{2}$ are the slope and constant of the baseline fit. The function parameters and lag times determined by this procedure are shown in Table 7.5.3.

\begin{tabular}{l|lll|ll} 
& Control & & anle138b & \\
\hline $\begin{array}{l}\text { Assay } \\
\text { Code: }\end{array}$ & ASS10 & ASS13 & ASS21 & ASS10 & ASS13 \\
\hline $\mathbf{m}_{\mathbf{1}}$ & 51.193 & 63.445 & 52.092 & 23.789 & 25.221 \\
$\mathbf{c}_{1}$ & -142.29 & -331.56 & -229.87 & -75.765 & -95.583 \\
$\mathbf{m}_{\mathbf{2}}$ & -0.4007 & 0.2123 & 0.0655 & 0.367 & -0.3382 \\
$\mathbf{C}_{2}$ & 8.578 & 19.767 & 9.5271 & 8.8243 & 13.532 \\
\hline $\mathbf{t}_{\text {lag }}$ & $\mathbf{2 . 9 2}$ & $\mathbf{5 . 5 6}$ & $\mathbf{4 . 6 0}$ & $\mathbf{3 . 6 1}$ & $\mathbf{4 . 2 7}$
\end{tabular}

Table 7.5.3 Fit parameters and lag times estimated for aggregation assays of $\alpha S$ in the presence of POPA/POPC vesicles in the presence and absence of anle138b

The oligomer concentration was estimated under the assumption, that the total protein concentration $[P]_{t}$ is the sum of the concentrations of the two observable species monomer $[M]_{t}$ and fibril $[F]_{t}$ and the non-observable species $[O]_{t}$ :

$$
[P]_{\text {tot }}=[M]_{t}+[O]_{t}+[F]_{\mathrm{t}},
$$

which after rearrangement gives the oligomer concentration as

$$
[O]_{t}=[P]_{t o t}-[M]_{t}-[F]_{t} .
$$

Under the assumption of relative concentrations $[P]_{\text {tot }}$ becomes 1 and equation (7-3) simplifies to

$$
[0]_{t}=1-[M]_{t}-[F]_{t} .
$$

The relative monomer concentration can be readily measured by solution-state NMR, by dividing the measured intensity $I(N M R)_{t}$ by the initial intensity $I(N M R)_{0}$, giving the normalized NMR intensity as

$$
\mathrm{I}(\mathrm{NMR})_{\mathrm{n}}=\frac{\mathrm{I}(\mathrm{NMR})_{\mathrm{t}}}{\mathrm{I}(\mathrm{NMR})_{0}}
$$


Since the NMR experiments showed, that the monomeric protein is never fully consumed in the observed time window, the observed fibril concentration at the end of the reaction is expressed by the equation

$$
[\mathrm{F}]_{\infty}=[P]_{\text {tot }}-[M]_{\infty}=1-[\mathrm{M}]_{\infty}
$$

where $[F]_{\infty}$ and $[M]_{\infty}$ are the observed final concentrations of fibrillar and monomeric species, implying the reaction has reached equilibrium. The normalized maximum intensity $I(F)_{n}$ was then calculated by subtracting the initial intensity $I(F)_{0}$, then dividing the individual intensities at time $t$ by the maximum recorded intensity $I(F)$ max and multiplying by the observed fibril concentration $[F]_{\infty}$, resulting in the equation

Min-max normalization ${ }^{[228]}$

$$
\mathrm{I}(\mathrm{F})_{\mathrm{n}}=\frac{\mathrm{I}(\mathrm{F})_{\mathrm{t}}-\mathrm{I}(\mathrm{F})_{0}}{\mathrm{I}(\mathrm{F})_{\max }-\mathrm{I}(\mathrm{F})_{0}} *\left(1-\frac{\mathrm{I}(\mathrm{NMR})_{\infty}}{\mathrm{I}(\mathrm{NMR})_{0}}\right)
$$

This operation ensures, that the normalized fluorescence intensities, representing the fibril concentration $[F]_{t}$ cannot reach $[P]_{t o t}$, as expected from the NMR measurements. The obtained data were used to plot the normalized intensities $I(N M R)_{n}$ and $I(F)_{n}$ over $t / t_{\text {lag }}$ and fitted to a sigmoidal function. For all sigmoidal fits to the monomeric data, the top asymptote was fixed at a value of 1 , for fits to fluorescence data, bottom asymptote was fixed to baseline values and top asymptote was set to $[F]_{\infty}$. Fitting to control samples did not result in a physically sensible plot, so that fits were only applied to values for $t / t_{a g} \leq 2$. This way, at least the initial part of the graph showed reasonable fitting.

Assuming that the relative monomer and fibril concentrations are measured by the normalized intensities $[M]_{t}=I(N M R)_{n}$ and $[F]_{t}=I(F)_{n}$, the oligomer concentration can be calculated by substitution into equation (7-4) as

$$
[0]_{t}=1-\mathrm{I}(\mathrm{NMR})_{n}-\mathrm{I}(\mathrm{F})_{n} .
$$

This equation was used to calculate relative oligomer concentrations and their time dependent development.

\subsubsection{Secondary Structure Calculation by BeStSel}

Secondary Structure Calculation was performed using the single spectrum analysis tool of BeStSel. ${ }^{[156]}$ Measured ellipticity data (mdeg) were submitted to the platform, along with the protein sample concentration $(5.8 \mu \mathrm{M})$ and path length $(0.1 \mathrm{~cm})$. Elipticities were converted 
into $\Delta \varepsilon$ values by the platform and secondary structure calculation was performed without application of a scaling factor by fitting from 190-250 nm. Fold recognition of secondary structure content was performed for samples at $t_{\text {lag }}$, and only the CATH class with the highest probability was chosen for further analysis. Experimental, along with fitted and residual data are annotated below for all individual samples.
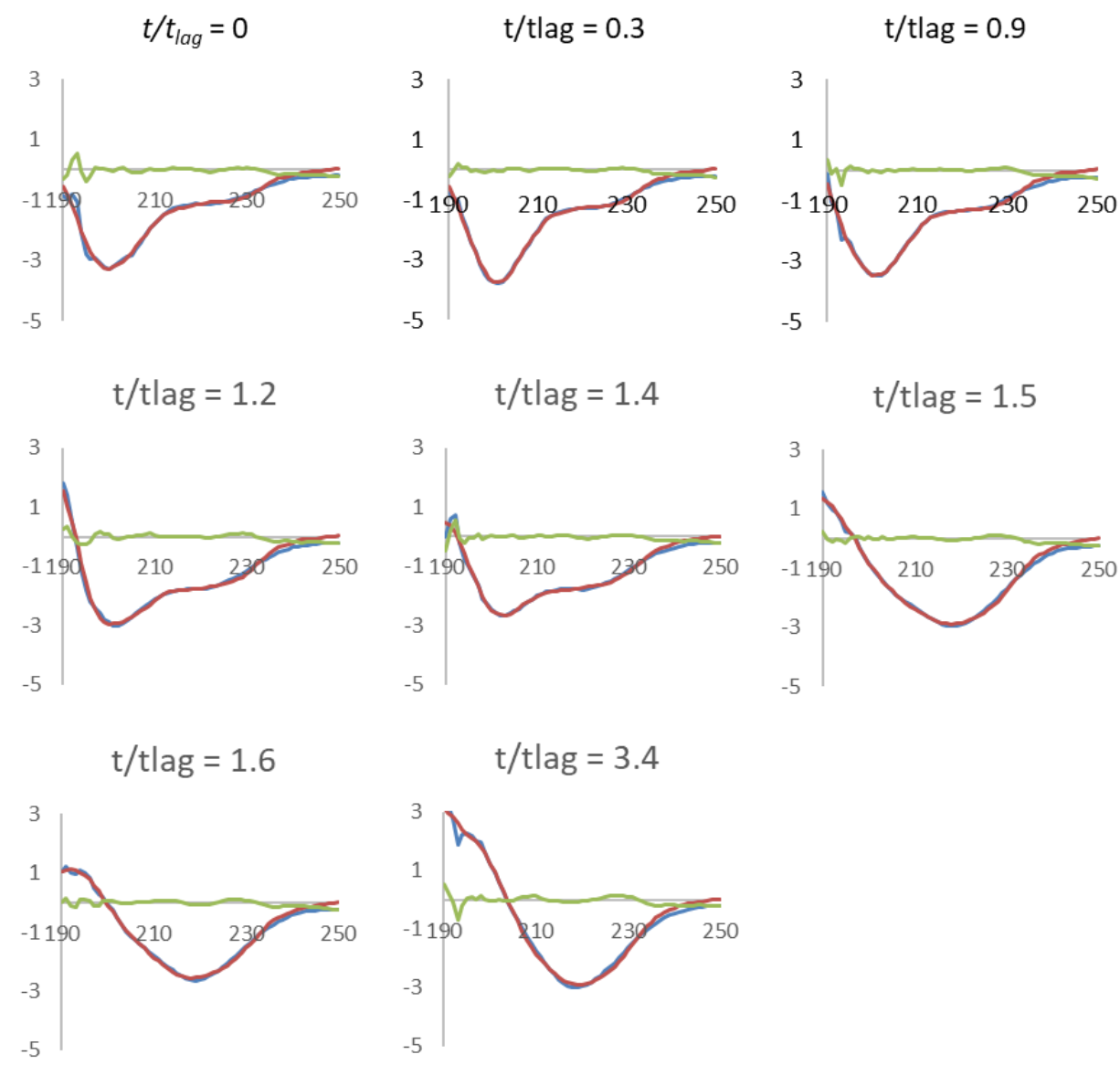

Figure 7.5.4 Experimental (blue), fitted (red) and residual data (green) returned by BeStSel for samples from PMCA experiments in the presence of phospholipids 

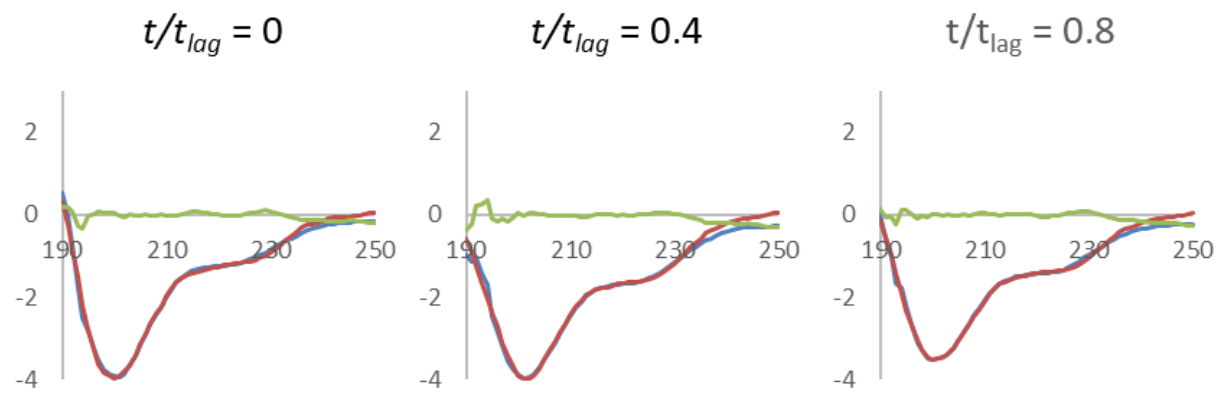

$$
\mathrm{t} / \mathrm{t}_{\text {lag }}=1.0
$$

$\mathrm{t} / \mathrm{t}_{\mathrm{lag}}=1.2$

$$
\mathrm{t} / \mathrm{t}_{\text {lag }}=1.3
$$
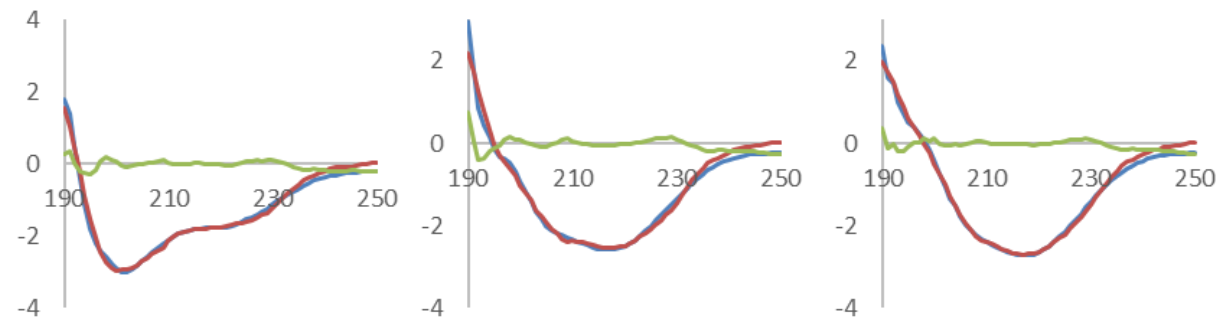

$$
\mathrm{t} / \mathrm{t}_{\text {lag }}=1.5
$$

$\mathrm{t} / \mathrm{t}_{\mathrm{lag}}=2.3$

$$
t / t_{\text {lag }}=6.7
$$
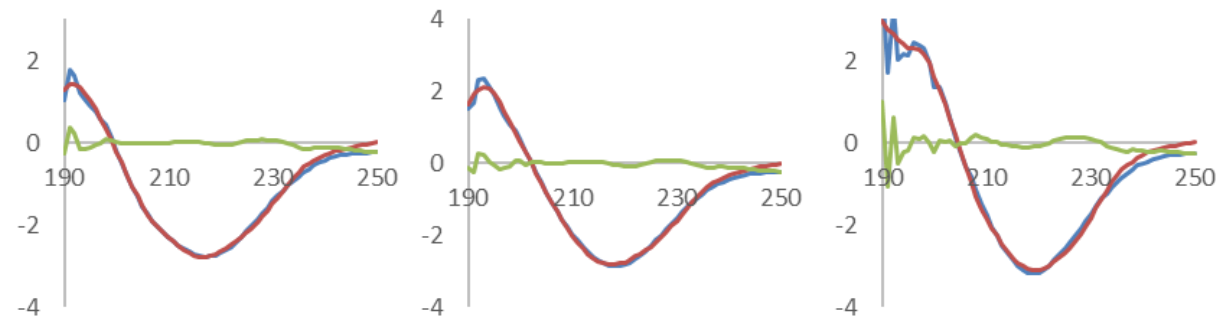

Figure 7.5.5 Experimental (blue), fitted (red) and residual data (green) returned by BeStSel for samples from PMCA experiments in the presence of phospholipids containing anle138b 


$$
t / t_{\text {lag }}=0
$$

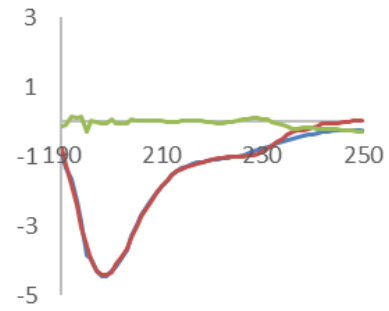

$t / t_{\text {lag }}=1.2$

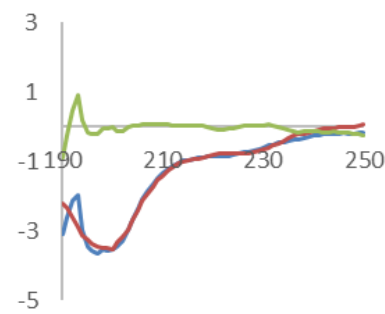

$t / t_{\text {lag }}=1.6$

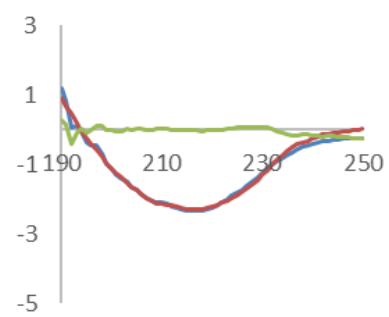

$t / t_{\text {lag }}=0.3$

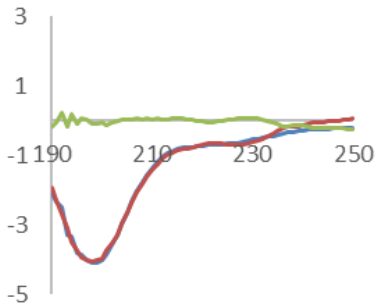

$t / t_{\text {lag }}=1.4$

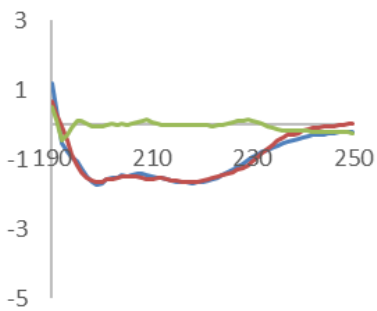

$t / t_{\text {lag }}=3.4$

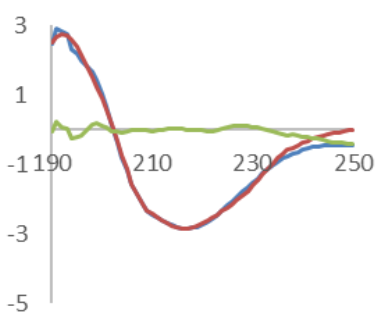

$t / t_{\text {lag }}=0.9$

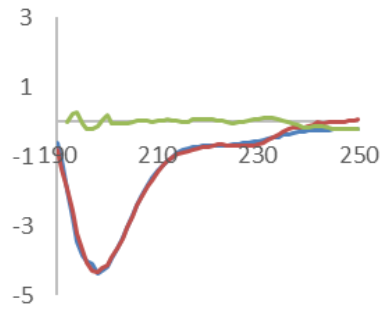

$t / t_{\text {lag }}=1.5$

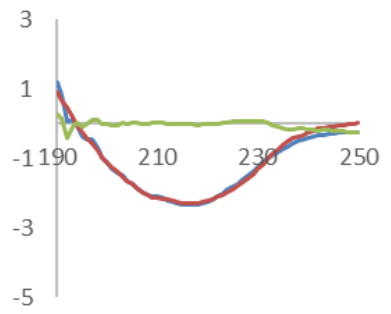

Figure 7.5.6 Experimental (blue), fitted (red) and residual data (green) returned by BeStSel for samples from PMCA experiments in the absence of phospholipids

\subsubsection{SsNMR}

For isolation of intermediates aggregation was monitored continuously by ThT fluorescence. As soon as an increase in fluorescence intensity was detected, incubation was halted by cooling samples on ice and immediately centrifuging at 55.000 rpm (TLA-100.3 rotor in an Optima $^{\mathrm{TM}} \mathrm{MAX}-\mathrm{TL}$ ) for $1 \mathrm{~h}$ at $4{ }^{\circ} \mathrm{C}$. For preparation of fibrils, aggregation was continued for $96 \mathrm{~h}$, followed by similar treatment. After removal of the supernatant, samples were washed with fresh buffer (5 mM HEPES, pH 7.4) and subsequently centrifuged (10 min, 65.000 rpm, $18^{\circ} \mathrm{C}$ ). Excess moisture was carefully removed, and samples were packed into ssNMR rotors by cutting off the bottom of the tube and directly centrifuging the pellet directly into the rotor of choice through a custom-made filling device. Finally, the rotor was then centrifuged for $30 \mathrm{~min}$ at $24.000 \mathrm{rpm}$ for packing (Beckman Coulter.....). For sequence assignment 
spectra [13C, 15N]- and [2H, 13C, 15N]-labelled aS were incubated with SUVs at a L/P ratio of 5 .

3D hCANH, hcoCAcoNH, hCONH, hCANH, hcaCBcaNH, hcaCBcacoNH experiments for protein sequence assignment were acquired using $[2 \mathrm{H}, 13 \mathrm{C}, 15 \mathrm{~N}]$-labelled $\mathrm{aS}$ on an 800 $\mathrm{MHz}$ Bruker Avance III spectrometer at a magnetic field of $18.8 \mathrm{~T}$ equipped with a $1.3 \mathrm{~mm}$ HRMAS HCN probe and MAS at $55 \mathrm{kHz}$. The temperature of the cooling gas was set to $250 \mathrm{~K}$, resulting in an estimated sample temperature of $20^{\circ} \mathrm{C}$.

Chemical Shift data for $13 \mathrm{CO}, 13 \mathrm{C} \alpha$ and $13 \mathrm{C} \beta$ obtained from sequence assignment spectra were used in TALOS+ to obtain predictions on secondary structure as well as dihedral backbone angles. ${ }^{[229]}$

All 2D 13C13C-DARR Spectra were acquired on an $850 \mathrm{MHz}$ Avance III with $3.2 \mathrm{~mm}$ HRMAS HCN probe at a magnetic field of $20.0 \mathrm{~T}$ and MAS at $17 \mathrm{kHz}$. DARR spectra for all samples were acquired with mixing times of $20 \mathrm{~ms}$. Further we acquired spectra with mixing times of $200 \mathrm{~ms}$ for fibrils and monomers as well as 5 and $50 \mathrm{~ms}$ for monomers.

Spectra were processed Spectra were acquired in short blocks, which were each corrected for linear drift of the static magnetic field using a script written by E. E. Najbauer and L. B. Andreas. ${ }^{[230]}$ Blocks for 2D spectra at $850 \mathrm{MHz}$ were either $3 \mathrm{~h}$ (for Intermediates) or $6 \mathrm{~h}$ (monomers and fibrils). Blocks for 3D spectra at 800 and $950 \mathrm{MHz}$ were either $24 \mathrm{~h}(\mathrm{hCONH}$, hCANH, hcaCBcaNH, hcaCBcacoNH) or $36 \mathrm{~h}$ (hCANH, hcoCAcoNH, HhNH). The drift corrected blocks were then averaged and processed as one spectrum. Spectra were analyzed using CcpNmr Analysis and NMRFAM-Sparky. ${ }^{[231]}$

In case of DNP measurements, the pellet was added by a solution of TEMTRIPOL in a solution of ${ }^{13} \mathrm{C}$-depleted $d_{8}$-glycerol and water (3:7), before centrifugation. Before injection into the ssNMR probe, rotors were plunge frozen in liquid $\mathrm{N}_{2}$ for glassification and thawed. This procedure was repeated twice.

\subsubsection{Estimation of $\alpha$ S per SUV}

The aggregation Number $\mathrm{N}$ represents the number of lipid molecules that make up an aggregated structure such as a micelle or an SUV. By estimation of the aggregation number trough simple geometric considerations, the local concentration of anle138b, depicting the concentration of the compound inside the bilayer was approximated. The calculations made in this work are based on considerations made in literature. ${ }^{[109]}$

Considering an SUV as a perfect sphere, its surface area can be expressed as the sum of the areas of the outer and the inner layer: 


$$
A_{\text {SUV }}=4 \pi r_{\text {out }}^{2}+4 \pi\left(r_{\text {out }}-d_{\text {bilayer }}\right)^{2}
$$

Here, $r_{\text {out }}$ is the hydrodynamic radius of the SUV, which is determined from DLS measurements. Knowing the surface area of the SUV, the aggregation number of the SUV can directly be obtained by

$$
N_{\text {aggr }}=\frac{A_{\text {SUV }}}{a_{0}},
$$

where $a_{0}$ is the area of a lipid head group. In the approach described here, it is assumed, that the headgroup area is represented by the average headgroup area for the two contributing lipids and that the head group area does not change depending on the position in the outer or inner leaflet. The head group area is thus derived as

$$
\mathrm{a}_{0}=\frac{\mathrm{a}_{0}(\operatorname{lipid} 1)+\mathrm{a}_{0}(\text { lipid } 2)}{2} .
$$

The total number of SUVs in a solution $\mathrm{N}_{\text {suv }}$ can be calculated, if all vesicles are assumed to be of the same size by

$$
N_{\text {SUV }}=\frac{n_{\text {lipid }}}{N_{\text {aggr }}},
$$

where $n_{\text {lipid }}$ is the total amount of substance for solution.

The number of aS molecules is then achieved by dividing the aS monomer concentration by the number of vesicles.

Area per lipid and bilayer thickness were adopted from comparable systems, giving $\mathrm{a}_{0}=0.56 \mathrm{~nm}^{2}$ and $d_{\text {bilayer }}=4.7 \mathrm{~nm}$. ${ }^{[232]}$ The membrane bound $\alpha \mathrm{S}$ fraction was estimated from the plateau regions in Figure 3.1.2 A.

\begin{tabular}{r|rrr} 
L/P & $\mathbf{5}$ & $\mathbf{1 0}$ & $\mathbf{2 0}$ \\
\hline [lipid] & 350 & 700 & 1400 \\
$\mathrm{~N}($ lipid $) / \mathrm{mL}$ & $2.1 \mathrm{E}+17$ & $4.2 \mathrm{E}+17$ & $8.4 \mathrm{E}+17$ \\
$\mathrm{~N}(\mathrm{SUV}) / \mathrm{ml}$ & $1.48 \mathrm{E}+13$ & $2.96 \mathrm{E}+13$ & $5.92 \mathrm{E}+13$ \\
Fraction of aS bound to lipid & 0.2 & 0.4 & 0.8 \\
\hline $\boldsymbol{\alpha S}$ per vesicle & $\mathbf{5 6 9}$ & $\mathbf{5 6 9}$ & $\mathbf{5 6 9}$
\end{tabular}




\section{Literature}

[1] P. A. Lewis, J. E. Spillane, in The Molecular and Clinical Pathology of Neurodegenerative Disease (Eds.: P. A. Lewis, J. E. Spillane), Academic Press, 2019, pp. 1-23.

[2] M.-T. Heemels, Nature 2016, 539, 179.

[3] G. Research, Global Neurodegenerative Diseases Drugs Market to 2023, https://www.researchandmarkets.com/research/sq4cpk/global?w=12, (accessed 23rd July 2019).

[4] J. Research, What Is Neurodegenerative Disease?, https://www.neurodegenerationresearch.eu/about/what/, (accessed 23rd July 2019).

[5] G. D. Cuny, Future Medicinal Chemistry 2012, 4, 1647-1649.

[6] K. A. Jellinger, J. Cell. Mol. Med. 2010, 14, 457-487.

[7] L. Migliore, F. Coppedè, Mutation Research/Fundamental and Molecular Mechanisms of Mutagenesis 2009, 667, 82-97.

[8] C. J. Chang, et al., Chem. Soc. Rev. 2014, 43, 6668-6671.

[9] K. S. Sheinerman, S. R. Umansky, Cell Cycle 2013, 12, 1-2.

[10] G. M. Sancesario, S. Bernardini, Annals of Translational Medicine 2018, 6.

[11] D. M. Skovronsky, et al., Annual Review of Pathology: Mechanisms of Disease 2006, 1, 151-170.

[12] J. Vaquer-Alicea, M. I. Diamond, Annu. Rev. Biochem 2019, 88, 785-810.

[13] M. Jucker, L. C. Walker, Nature 2013, 501, 45.

[14] J. Y. Lee, et al., Neurodegenerative diseases 2015, 15, 339-349.

[15] F. Chiti, C. M. Dobson, Annu. Rev. Biochem 2017, 86, 27-68.

[16] C. G. Chung, et al., Cell. Mol. Life Sci. 2018, 75, 3159-3180.

[17] P. Arosio, et al., PCCP 2015, 17, 7606-7618.

[18] J. Féger, E. C. Hirsch, Annales Pharmaceutiques Françaises 2015, 73, 3-12.

[19] Reuters, Pfizer ends research for new Alzheimer's, Parkinson's drugs, https://www.reuters.com/article/us-pfizer-alzheimers/pfizer-ends-research-for-newalzheimers-parkinsons-drugs-idUSKBN1EWOTN, (accessed 24th July 2019).

[20] J. Voss, et al., Future Medicinal Chemistry 2012, 4, 1661-1669.

[21] J. J. Mendoza-Velásquez, et al., Frontiers in Neurology 2019, 10, 363.

[22] R. M. Giráldez-Pérez, et al., Acta Neuropathologica Communications 2014, 2, 176.

[23] S. M. Goldman, Annu. Rev. Pharmacool. Toxicol. 2014, 54, 141-164.

[24] J. Jankovic, Journal of Neurology, Neurosurgery \&amp;amp; Psychiatry 2008, 79, 368.

[25] R. A. Barker, C. H. Williams-Gray, Neuropathol. Appl. Neurobiol. 2016, 42, 6-19.

[26] E. Dantuma, et al., Stem Cell Research \& Therapy 2010, 1, 37.

[27] H. McCann, et al., Parkinsonism \& Related Disorders 2014, 20, S62-S67.

[28] S. H. Shahmoradian, et al., Nat. Neurosci. 2019, 22, 1099-1109.

[29] M. G. Spillantini, et al., Nature 1997, 388, 839-840.

[30] D. B. Hogan, et al., Canadian Journal of Neurological Sciences / Journal Canadien des Sciences Neurologiques 2016, 43, S83-S95.

[31] M. C. Mayo, Y. Bordelon, Semin Neurol 2014, 34, 182-188.

[32] Z. Walker, et al., The Lancet 2015, 386, 1683-1697.

[33] D. Hansen, et al., Neuropathol. Appl. Neurobiol. 2019, 0.

[34] N. Stefanova, G. K. Wenning, Neuropathol. Appl. Neurobiol. 2016, 42, 20-32.

[35] G. K. Wenning, F. Krismer, in Handbook of Clinical Neurology, Vol. 117 (Eds.: R. M. Buijs, D. F. Swaab), Elsevier, 2013, pp. 229-241.

[36] S. Gilman, et al., Neurology 2008, 71, 670.

[37] A. J. Hughes, et al., Journal of neurology, neurosurgery, and psychiatry 1992, 55, 1009-1013.

[38] N. L.-G. Del Rey, et al., Frontiers in neuroanatomy 2018, 12, 113-113.

[39] S. D. Capouch, et al., Neurology and Therapy 2018, 7, 249-263.

[40] S. Maaß, et al., Current Treatment Options in Neurology 2016, 18, 51. 
[41] T. Gasser, Expert Reviews in Molecular Medicine 2009, 11, e22.

[42] M. L. Kringelbach, et al., Nature Reviews Neuroscience 2007, 8, 623.

[43] A. A. Kühn, J. Volkmann, Movement Disorders 2017, 32, 11-19.

[44] G. K. Wenning, et al., Journal of Neurology, Neurosurgery \&amp;amp; Psychiatry 2000, 68, 434.

[45] I. Alafuzoff, P. Hartikainen, in Handbook of Clinical Neurology, Vol. 145 (Eds.: G. G. Kovacs, I. Alafuzoff), Elsevier, 2018, pp. 339-353.

[46] H. Chen, B. Ritz, Journal of Parkinson's disease 2018, 8, S9-S17.

[47] E. Sturm, N. Stefanova, Exp Neurobiol 2014, 23, 277-291.

[48] K. Wirdefeldt, et al., Eur. J. Epidemiol. 2011, 26, 1.

[49] L. Tagliafierro, O. Chiba-Falek, Up-regulation of SNCA gene expression: implications to synucleinopathies 2016, 17, 145-157.

[50] M. G. Spillantini, M. Goedert, Neuropathol. Appl. Neurobiol. 2016, 42, 3-5.

[51] D. J. Irwin, et al., Nature Reviews Neuroscience 2013, 14, 626.

[52] T. Ozawa, et al., Brain 2004, 127, 2657-2671.

[53] G. Monzio Compagnoni, A. Di Fonzo, Acta Neuropathologica Communications 2019, 7, 113.

[54] L. Klingelhoefer, H. Reichmann, Nature Reviews Neurology 2015, 11, 625.

[55] D. Sulzer, R. H. Edwards, J. Neurochem. 2019, 0.

[56] R. F. Roberts, et al., Brain 2015.

[57] C. Soto, S. Pritzkow, Nat. Neurosci. 2018, 21, 1332-1340.

[58] C. Peng, et al., Nature 2018, 557, 558-563.

[59] T. R. Yamasaki, et al., J. Biol. Chem. 2019, 294, 1045-1058.

[60] R. S. Pagano, et al., Biophys. J. 2014, 107, 711-720.

[61] H. Flyvbjerg, et al., Proc. Natl. Acad. Sci. U.S.A. 1996, 93, 5975.

[62] G. T. Debelouchina, et al., PCCP 2010, 12, 5911-5919.

[63] W. R. Markesbery, et al., J. Neuropathol. Exp. Neurol. 2009, 68, 816-822.

[64] A. J. Espay, et al., Neurology 2019, 92, 329.

[65] P. Modrego, A. Lobo, Neurodegenerative Disease Management 2019, 9, 119-121.

[66] A. C. M. Ferreon, et al., Proceedings of the National Academy of Sciences 2009, 106, 5645.

[67] B. G. Wilhelm, et al., Science 2014, 344, 1023.

[68] B. Mollenhauer, et al., Exp. Neurol. 2008, 213, 315-325.

[69] L. Breydo, et al., Biochimica et Biophysica Acta (BBA) - Molecular Basis of Disease 2012, 1822, 261-285.

[70] J. T. Bendor, et al., Neuron 2013, 79, 1044-1066.

[71] A. Gámez-Valero, K. Beyer, Genes 2018, 9.

[72] D. F. Clayton, J. M. George, J. Neurosci. Res. 1999, 58, 120-129.

[73] K. Beyer, Acta Neuropathol. 2006, 112, 237-251.

[74] V. N. Uversky, J. Neurochem. 2007, 103, 17-37.

[75] D. F. Clayton, J. M. George, Trends Neurosci. 1998, 21, 249-254.

[76] V. N. Uversky, et al., Chem. Rev. 2014, 114, 6844-6879.

[77] P. Bernadó, et al., J. Am. Chem. Soc. 2005, 127, 17968-17969.

[78] T. Bartels, et al., Nature 2011, 477, 107.

[79] E. Coelho-Cerqueira, et al., The FEBS journal 2013, 280, 4915-4927.

[80] F.-X. Theillet, et al., Nature 2016, 530, 45.

[81] H. A. Lashuel, et al., Nature reviews. Neuroscience 2013, 14, 38-48.

[82] V. N. Uversky, et al., J. Biol. Chem. 2001, 276, 10737-10744.

[83] M.-K. Cho, et al., Protein science : a publication of the Protein Society 2009, 18, 1840-1846.

[84] H. Roberts, D. Brown, Biomolecules 2015, 5, 282.

[85] L. Breydo, V. N. Uversky, FEBS Lett. 2015, 589, 2640-2648.

[86] N. Cremades, et al., in International Review of Cell and Molecular Biology, Vol. 329 (Ed.: M. Sandal), Academic Press, 2017, pp. 79-143.

[87] F. van Diggelen, et al., Isr. J. Chem. 2017, 57, 699-723. 
[88] N. Lorenzen, et al., J. Am. Chem. Soc. 2014, 136, 3859-3868.

[89] S. W. Chen, et al., Proc. Natl. Acad. Sci. U.S.A. 2015, 112, E1994-E2003.

[90] G. Fusco, et al., Science 2017, 358, 1440-1443.

[91] G. Comellas, et al., J. Am. Chem. Soc. 2012, 134, 5090-5099.

[92] W. Paslawski, et al., Characterization of hydrophobic residue requirements for alphasynuclein fibrillization 2014, 53, 6252-6263.

[93] H.-Y. Kim, et al., J. Am. Chem. Soc. 2009, 131, $17482-17489$.

[94] M. T. Stöckl, et al., Mol. Neurobiol. 2013, 47, 613-621.

[95] H. Chaudhary, et al., Langmuir 2016, 32, 11827-11836.

[96] D. N. Bloch, Y. Miller, ACS Omega 2017, 2, 3363-3370.

[97] M. D. Tuttle, et al., Nat. Struct. Mol. Biol. 2016, 23, 409.

[98] R. Guerrero-Ferreira, et al., bioRxiv 2018.

[99] B. Li, et al., Nat. Comm. 2018, 9, 3609.

[100] R. Guerrero-Ferreira, et al., bioRxiv 2019, 654582.

[101] Y. Li, et al., Cell Res. 2018, 28, 897-903.

[102] A. W. P. Fitzpatrick, et al., Nature 2017, 547, 185.

[103] B. Falcon, et al., Nature 2018, 561, 137-140.

[104] D. P. Karpinar, et al., The EMBO Journal 2009, 28, 3256-3268.

[105] D. S. Eisenberg, M. R. Sawaya, Annu. Rev. Biochem 2017, 86, 69-95.

[106] N. Plotegher, et al., Q. Rev. Biophys. 2014, 47, 1-48.

[107] J. E. Gillam, C. E. MacPhee, J. Phys.: Condens. Matter 2013, 25, 373101.

[108] A. K. Buell, et al., Proc. Natl. Acad. Sci. 2014, 111, 7671-7676.

[109] C. Galvagnion, et al., Nat. Chem. Biol. 2015, 11, 229-234.

[110] C. Galvagnion, et al., Proc. Natl. Acad. Sci. 2016.

[111] H.-J. Lee, et al., J. Biol. Chem. 2002, 277, 671-678.

[112] E. R. Middleton, E. Rhoades, Biophys. J. 2010, 99, 2279-2288.

[113] T. Bartels, et al., Biophys. J. 2010, 99, 2116-2124.

[114] Y. Zarbiv, et al., Neurobiology of Disease 2014, 70, 90-98.

[115] G. Fusco, et al., 2014, 5, 3827.

[116] M. Drescher, et al., ChemBioChem 2008, 9, 2411-2416.

[117] E. R. Georgieva, et al., J. Am. Chem. Soc. 2008, 130, 12856-12857.

[118] T. Viennet, et al., Communications Biology 2018, 1, 44.

[119] G. P. Saborio, et al., Nature 2001, 411, 810-813.

[120] N. Salvadores, et al., Cell Reports 2014, 7, 261-268.

[121] V. Meyer, et al., Characterization of hydrophobic residue requirements for alphasynuclein fibrillization 2014, 53, 5804-5809.

[122] M. E. Herva, et al., J. Biol. Chem. 2014, 289, 11897-11905.

[123] S. Paciotti, et al., Frontiers in Neurology 2018, 9.

[124] M. A. Barria, et al., in Amyloid Proteins: Methods and Protocols (Eds.: E. M.

Sigurdsson, M. Calero, M. Gasset), Humana Press, Totowa, NJ, 2012, pp. 199-212.

[125] J. Masel, et al., Biophys. Chem. 1999, 77, 139-152.

[126] A. Fenyi, et al., Neurobiology of Disease 2019, 129, 38-43.

[127] U. J. Kang, et al., Movement Disorders 2019, 34, 536-544.

[128] J.-H. Park, et al., Mol. Neurobiol. 2018, 55, 1630-1638.

[129] R. Atarashi, et al., Real-time quaking-induced conversion: a highly sensitive assay for prion detection 2011, 5, 150-153.

[130] A. Inc., https://www.amprionme.com/breaking-news-amprion-receives-fdabreakthrough-device-designation-for-detection-of-alpha-synuclein/, (accessed 19th July 2019 2019).

[131] J. Wagner, et al., Acta Neuropathol. 2013, 125, 795-813.

[132] A. Heras-Garvin, et al., Movement Disorders 2019, 34, 255-263.

[133] L. Fellner, et al., Frontiers in Neuroscience 2016, 10.

[134] M. Wegrzynowicz, et al., Acta Neuropathol. 2019.

[135] A. Martinez Hernandez, et al., EMBO Molecular Medicine 2018, 10, 32-47.

[136] J. Wagner, et al., Acta Neuropathol. 2015, 130, 619-631. 
[137] A. A. Deeg, et al., Biochim. Biophys. Acta 2015, 1850, 1884-1890.

[138] A. M. Reiner, et al., Biochimica et Biophysica Acta (BBA) - General Subjects 2018, 1862, 800-807.

[139] D. Matthes, et al., ACS Chemical Neuroscience 2017, 8, 2791-2808.

[140] G. S. Nyamato, et al., Acta Crystallographica Section C 2014, 70, 780-783.

[141] H. A. Scheidt, D. Huster, Acta Pharmacol Sin 2008, 29, 35-49.

[142] M. Zhu, A. L. Fink, J. Biol. Chem. 2003, 278, 16873-16877.

[143] G. Liu, et al., ACS Medicinal Chemistry Letters 2012, 3, 856-859.

[144] C. R. Bodner, et al., J. Mol. Biol. 2009, 390, 775-790.

[145] S. Eugène, et al., The Journal of Chemical Physics 2016, 144, 175101.

[146] P. Arosio, et al., Trends Pharmacol. Sci. 2014, 35, 127-135.

[147] Rodrigo S. Pagano, et al., Biophys. J. 2014, 107, 711-720.

[148] M. Iljina, et al., Proc. Natl. Acad. Sci. 2016, 113, E1206-E1215.

[149] A. Quist, et al., Proc. Natl. Acad. Sci. U.S.A. 2005, 102, 10427-10432.

[150] H. Ruf, et al., in Methods Enzymol., Vol. 172, Academic Press, 1989, pp. 364-390.

[151] B. A. Cornell, et al., Biochimica et Biophysica Acta (BBA) - Biomembranes 1982, 690, 15-19.

[152] R. P. Richter, et al., Langmuir 2006, 22, 3497-3505.

[153] B. A. Wallace, Q. Rev. Biophys. 2010, 42, 317-370.

[154] A. Micsonai, et al., Nucleic Acids Res. 2018, 46, W315-W322.

[155] A. Micsonai, et al., Proc. Natl. Acad. Sci. 2015, 112, E3095.

[156] BeStSel, http://bestsel.elte.hu/index.php, (accessed 11th Sept 2019).

[157] J. R. Allison, et al., J. Am. Chem. Soc. 2009, 131, 18314-18326.

[158] O. Ullman, et al., J. Am. Chem. Soc. 2011, 133, 19536-19546.

[159] M. Schwalbe, et al., Structure 2014, 22, 238-249.

[160] María S. Celej, et al., Biochem. J 2012, 443, 719-726.

[161] G. Comellas, et al., J. Mol. Biol. 2011, 411, 881-895.

[162] W. Close, et al., Nat. Comm. 2018, 9, 699.

[163] J. Gath, et al., Biomolecular NMR Assignments 2014, 8, 395-404.

[164] J. Verasdonck, et al., Biomolecular NMR Assignments 2016, 10, 5-12.

[165] A. M. Barclay, et al., Biomolecular NMR Assignments 2018, 12, 195-199.

[166] J. Gath, et al., Biomolecular NMR Assignments 2012, 6, 51-55.

[167] D. Ami, et al., Microbial cell factories 2013, 12, 17-17.

[168] Y. Ni, et al., Analyst 2011, 136, 4794-4801.

[169] M. T. Larsen, et al., Molecular and cellular therapies 2016, 4, 3-3.

[170] N. Alsabeeh, et al., Biochimica et biophysica acta. Molecular and cell biology of lipids 2018, 1863, 143-151.

[171] D. Ray, et al., The Journal of Physical Chemistry B 2015, 119, 2168-2179.

[172] V. N. Uversky, et al., FEBS Lett. 2002, 515, 99-103.

[173] S. Schenkman, et al., Chem. Phys. Lipids 1981, 28, 165-180.

[174] F. Ruggeri, et al., Soft Matter 2013, 9, 4219-4226.

[175] C.-J. Hsieh, et al., ACS Chemical Neuroscience 2018, 9, 2521-2527.

[176] K.-N. Hu, et al., The Journal of Chemical Physics 2008, 128, 052302.

[177] E. Smith, I. Collins, Future medicinal chemistry 2015, 7, 159-183.

[178] B. Q. Tran, et al., Biochimica et Biophysica Acta (BBA) - Proteins and Proteomics 2016, 1864, 123-129.

[179] M. S. Platz, Photochem. Photobiol. 1997, 65, 193-194.

[180] G. Carlos Augusto, Curr. Med. Chem. 2003, 10, 671-689.

[181] H. Inui, et al., J. Am. Chem. Soc. 2013, 135, 10246-10249.

[182] Y. Xia, L. Peng, Chem. Rev. 2013, 113, 7880-7929.

[183] P. J. Salveson, et al., J. Am. Chem. Soc. 2016, 138, 4458-4467.

[184] T. S. Ulmer, et al., J. Biol. Chem. 2005, 280, 9595-9603.

[185] S. Nicot, et al., The FASEB Journal 2019, fj.201900354R.

[186] T. F. Outeiro, et al., Molecular Neurodegeneration 2019, 14, 5.

[187] Z. Yuan, et al., J. Neurochem. 2015, 133, 722-729. 
[188] L. Giehm, et al., Proc. Natl. Acad. Sci. 2011, 108, 3246.

[189] N. Cremades, et al., Cell 2012, 149, 1048-1059.

[190] H. A. Lashuel, et al., J. Mol. Biol. 2002, 322, 1089-1102.

[191] G. Bhak, et al., PLOS ONE 2009, 4, e4177.

[192] R. E. Apfel, in Methods in Experimental Physics, Vol. 19 (Ed.: P. D. Edmonds), Academic Press, 1981, pp. 355-411.

[193] M. So, et al., Curr. Opin. Struct. Biol. 2016, 36, 32-39.

[194] M. A. A. Fakhree, et al., Scientific Reports 2016, 6, 30658.

[195] A. R. Braun, et al., J. Am. Chem. Soc. 2012, 134, 2613-2620.

[196] R. M. Meade, et al., Molecular Neurodegeneration 2019, 14, 29.

[197] A. A. Doinikov, A. Bouakaz, The Journal of the Acoustical Society of America 2010, 128, 11-19.

[198] A. A. Karimi Zarchi, et al., Nanomedicine Journal 2018, 5, 127-137.

[199] A. lyer, et al., J. Biol. Chem. 2016, 291, 21110-21122.

[200] M. A. A. Fakhree, et al., Biomacromolecules 2019, 20, 1217-1223.

[201] Z. Shi, et al., Physical chemistry chemical physics : PCCP 2015, 17, 15561-15568.

[202] V. H. Man, et al., The Journal of Chemical Physics 2019, 150, 215101.

[203] J. Kubelka, et al., Curr. Opin. Struct. Biol. 2004, 14, 76-88.

[204] E. Chatani, N. Yamamoto, Biophysical reviews 2018, 10, 527-534.

[205] C. Xue, et al., Royal Society Open Science 2017, 4, 160696.

[206] B. O'Nuallain, et al., Characterization of hydrophobic residue requirements for alphasynuclein fibrillization 2005, 44, 12709-12718.

[207] T. Urbic, et al., Biophys. Chem. 2017, 231, 155-160.

[208] H. Heise, et al., Interplay of alpha-synuclein binding and conformational switching probed by single-molecule fluorescence 2005, 102, 15871.

[209] M. D. Tuttle, et al., in Protein Amyloid Aggregation: Methods and Protocols (Ed.: D. Eliezer), Springer New York, New York, NY, 2016, pp. 173-183.

[210] E. Hellstrand, et al., PLOS ONE 2013, 8, e77235.

[211] E. A. Waxman, et al., Characterization of hydrophobic residue requirements for alpha-synuclein fibrillization 2009, 48, 9427-9436.

[212] L. Bédard, et al., Characterization of hydrophobic residue requirements for alphasynuclein fibrillization 2014, 53, 6463-6472.

[213] B. I. Giasson, et al., J. Biol. Chem. 2001, 276, 2380-2386.

[214] J. W. P. Brown, et al., Scientific Reports 2016, 6, 36010.

[215] M. M. Ouberai, et al., J. Biol. Chem. 2013, 288, 20883-20895.

[216] I. F. Tsigelny, et al., The FEBS Journal 2012, 279, 1000-1013.

[217] Y. Singh, et al., Chemistry - A European Journal 2011, 17, 151-160.

[218] N. Zijlstra, et al., Angew. Chem. Int. Ed. 2012, 51, 8821-8824.

[219] W. Paslawski, et al., Angew. Chem. Int. Ed. 2014, 53, 7560-7563.

[220] J. Levin, et al., Acta Neuropathol. 2014, 127, 779-780.

[221] V. Vasquez, et al., Journal of Alzheimer's disease : JAD 2018, 66, 453-460.

[222] T. Skamris, et al., Scientific Reports 2019, 9, 1733.

[223] B. Mannini, et al., ACS Chemical Biology 2014, 9, 2309-2317.

[224] A. M. Hernandez, et al., EMBO Molecular Medicine 2018, 10, 32-47.

[225] A. Balupuri, et al., Scientific Reports 2019, 9, 59.

[226] H. Yu, et al., The Journal of Chemical Physics 2015, 143, 243142.

[227] S. A. Hudson, et al., The FEBS Journal 2009, 276, 5960-5972.

[228] X. H. Cao, et al., BMC Bioinformatics 2016, 17, 359-359.

[229] Y. Shen, et al., J. Biomol. NMR 2009, 44, 213-223.

[230] E. E. Najbauer, L. B. Andreas, J. Magn. Reson. 2019, 305, 1-4.

[231] W. Lee, et al., Bioinformatics 2014, 31, 1325-1327.

[232] U. Kwolek, et al., The Journal of Physical Chemistry B 2015, 119, 10042-10051. 


\section{Appendix}

Pulse Sequences used in this work:

\section{${ }^{1} \mathrm{H}-1 \mathrm{D}$ NMR}

;zgpr.eth

; 1D sequence with presaturation

; modified gsw0203

;d1 : relaxation delay and water suppression

;pl1 : power for $1 \mathrm{H}$

;p21 : $1 \mathrm{~ms}$ (Gradient before acquisition)

;gpz1 : $50 \%$

;pl9 : power level for presaturation

;p1 : 90 degree hard pulse 1H

\#include <Avance_dl.incl>

1 ze
2 10u pl9:f1
10u LOCKH_OFF
d1 cw:f1
10u do:f1
10u pl1:f1
10u LOCKH_ON
p21:gp1
10m
p1 ph1
go=2 ph0
wr \#0
10u LOCKH_OFF
exit

ph1=0123

ph0=0123

;\#\#/(\$P[1],\$PL[1])=\&SetPulse(f1,HP,90); 


\section{${ }^{1} \mathrm{H}-{ }^{15} \mathrm{~N}-\mathrm{HSQC}$}

;hsqc15N.new

;D. Lee, Nov. 2002

;15N-1H HSQC correlations without water saturation

;The delay for 3-9-19 watergate (d5) should be matched ; with $1 / \mathrm{d}$;d=distance of next null point (in $\mathrm{Hz}$ ).

;S. Mori et al, JMR B108, 94-98 (1995)

;pl1 : power for $\mathbf{1 H}$

;pl2 : power for 13C

;pl3 : power for $15 \mathrm{~N}$

;pl13 : power for $15 \mathrm{~N}$ waltz16 decoupling

;p1 : 90 degree hard pulse $1 \mathrm{H}$

;p3 : 90 degree hard pulse 13C

;p4 : 180 degree hard $13 \mathrm{C}$ pulse (225d for $5 / 600)$

;p5 : 90 degree hard pulse 15N

;pcpd3 : 90 deg cpd-pulse15N(waltz16,160u)

;d1 : relaxation delay

;d2 : INEPT delay ( 2.7m)

;d5 : delay for 3-9-19=1/( $\mathrm{Hz}$ between nulls)

;in0 : 1/(2 SW) (Hz)

;p21 : 500u (Gradient in first INEPT)

;p22 : 500u (Gradient for z-filter)

;p23 : 1m (Gradient for second INEPT)

;gpz1 : 19\%

;gpz2 : $30 \%$

;gpz3 : 65\%

\#include <Avance kw.incl>

define delay INEPT_W

define delay INEPT_D 
\#define GRADIENT1 10u p21:gp1 200u

\#define GRADIENT2 10u p22:gp2 200u

\#define GRADIENT3 10u p23:gp3 200u

"p2=2*p1"

"p6=2*p5"

"in0=inf1/2"

"d0=in0/2-p5`2/3.14159-p1"

"d3=d5/2-p5"

"INEPT_D=d2-p21-210u"

"INEPT_W=d2-(p23+210u+p1 $\left.{ }^{\star 2} 2.3846+d 5^{\star} 2.5\right) "$

1 10u ze

2 1m do:f3

d1 pl1:f1

20u pl3:f3

20u LOCKH_ON

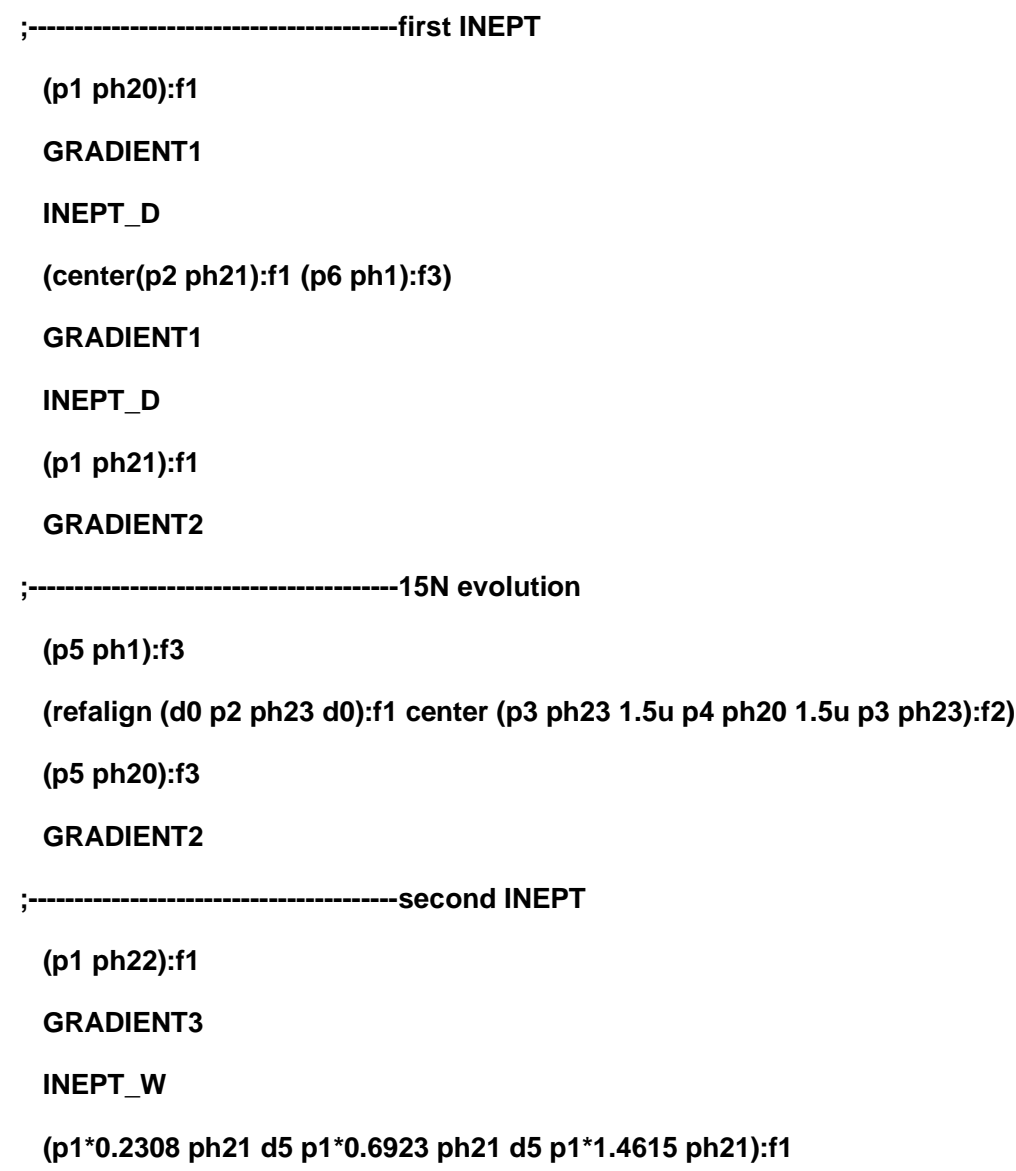




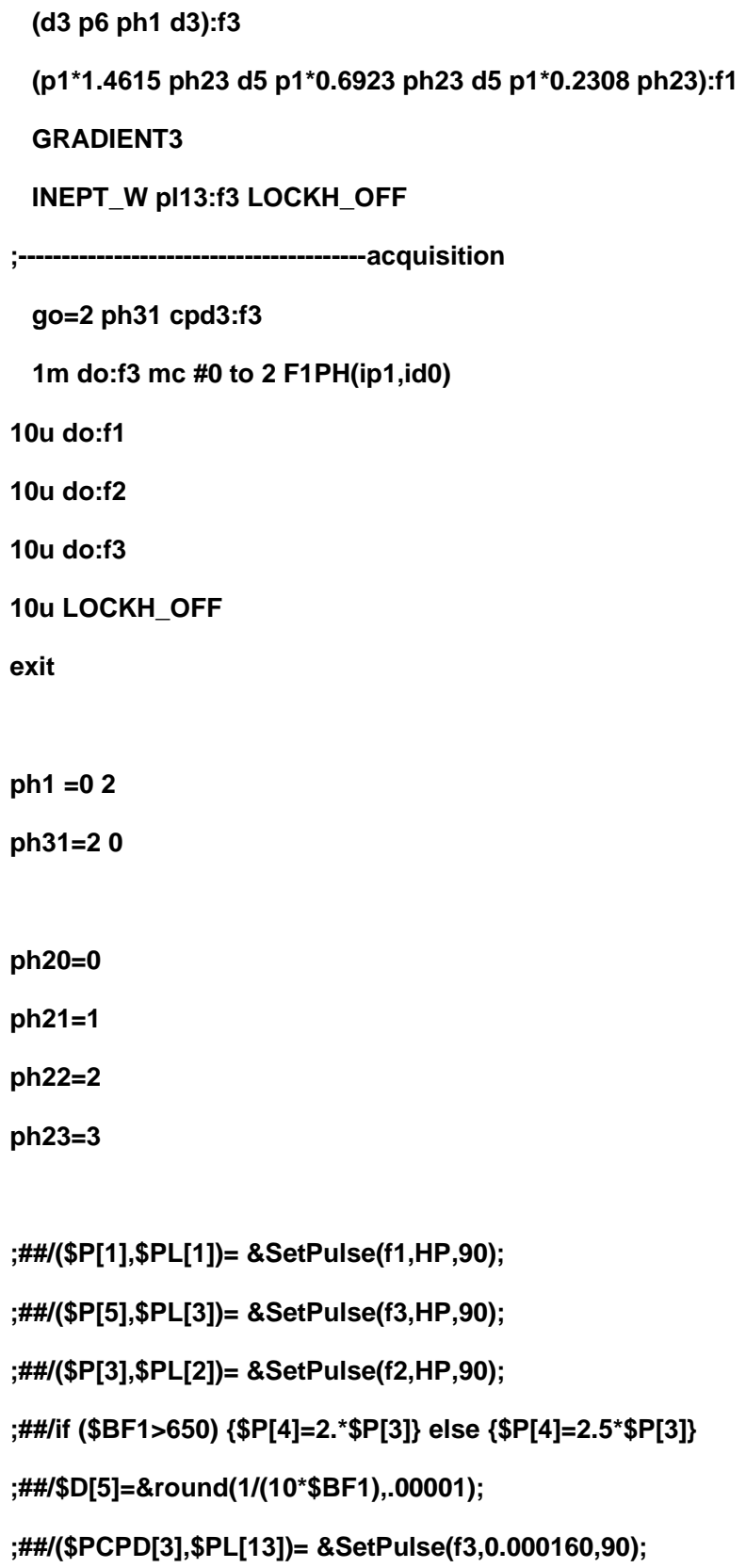

\section{$\underline{1 \mathrm{H}-13 \mathrm{C}-\mathrm{CP}}$}

;сp

;TS3 / 03.06.2011

;basic cp experiment

;written by HF 1.3.2001

;changed by JOS 05/06/03

;comments added by STE 4.4.2006 and HF 16.06.2010

;checked by SEWE 03.06.2011

;Avance III version

;parameters: 


\section{Appendix}

;p3 : proton 90 at power level PLW12

;p15: contact time at PLW1(f1) and SPW0(f2)

;pl1 : X power level during contact

;pl2 : =0W, not used

;pl12 : decoupling power level (if not PLW13)

;pl13 : special decoupling power level

;sp0 : proton power level during contact

;cnst21: on resonance, usually $=0$

;cpdprg2 : e.g. cw, spinal64 (at PLW12)

;d1 : recycle delay

;pcpd2 : pulse length in decoupling sequence (e.g. 180deg)

;spnam0 : use e.g. ramp.100 or ramp90100.100 for variable amplitude CP

;zgoptns : -Dfslg, -Dlacq, -Dlcp15, or blank

;\$CLASS=Solids

;\$IM=1D

\$TYPE=cross polarisation

;\$SUBTYPE=simple 1D

;\$COMMENT=basic $\mathrm{cp}$ experiment, arbitrary contact and decoupling schemes

prosol relations $=<$ solids_ $\mathrm{cp}>$

\#include <Avancesolids.incl $>$

\#ifdef fslg

\#include <lgcalc.incl>

;cnst20 : RF field achieved at pl13

;cnst21: on resonance, usually $=0$

;cnst22 : positive LG offset

;cnst23 : negative LG offset

;cnst24 : additional LG-offset

\#endif / ${ }^{*}$ fslg */

"acqt $0=0$; defines $\mathrm{t}=0$ for baseopt

1 ze

2 d1 do:f2

\#ifndef Icp15

\#include <p15_prot.incl>

;make sure p15 does not exceed $10 \mathrm{msec}$ ;let supervisor change this pulseprogram if ;more is needed

\#endif

\#ifndef lacq

;disable protection file for long acquisition change decoupling power !!! or you risk probe damage ;if you set the label lacq (ZGOPTNS -Dlacq), the protection is disabled

\#include <aq_prot.incl>

;allows max. 50 msec acquisition time, supervisor ;may change to max. $1 \mathrm{~s}$ at less than $5 \%$ duty cycle

\#endif ;and reduced decoupling field

$1 \mathrm{u} f \mathrm{q}=\mathrm{cnst} 21: \mathrm{f} 2$

(p3 pl12 ph1):f2

(p15 pl1 ph2):f1 (p15:sp0 ph10):f2

1u cpds2:f2 ;pl12 is used here with tppm, spinal, pl13 with cwlg, cwlgs

go=2 ph31

$1 \mathrm{~m}$ do:f2 
wr \#0

HaltAcqu, $1 \mathrm{~m}$

exit

$\mathrm{ph} 0=0$

ph1 $=13$

ph2= 00221133

ph10 $=0$

ph31= 02201331

;\$id: \$

\section{${ }^{13} \mathrm{C}^{13} \mathrm{C}$ RDFR}

; cpXdec.suva

; written on 120531

; modified on 120601

;ramp/tangential Cross-polarization

;decoulpling during acquisition

\section{; COMPILED FOR TOPSPIN 3.2}

$;================+=$
; Variables introduction

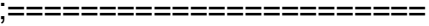

;d8 : pdsd mixing

;pl2 H 90 pulse power

;p2 H 90 pulse length

;P15 CP build up time

;pl5 (=sp1) CP power on X

;sp1 (=pl5) shape power o $X$

;pl6 (=sp0) CP power on $\mathrm{H}$

;sp0 (=pl6) shape power o $\mathrm{H}$

;cnst10 CP offset on $X$

;cnst20 CP offset on $\mathrm{H}$

;I31 (=2) ramp on $\mathrm{H}(=1)$ ramp on $\mathrm{X}$

;pl1 X 90 calibration pulse power

;p1 X 90 calibration pulse length

;d21 RFDR mixing time

;pl12 $\mathrm{H}$ decoupling during acquisition

;cpd2 decoupling program

;pcpd2 decoupling pulse length

;echo T2 echo time

;==============

; Set variables

;=========-=="

"cnst63 = plw12"

"acqt0 = 0"

"d9 = (1s/(2*cnst31)) - p11"

"d21 = I1 *(1s/cnst31)"

;"d0=l1*(1/cnst31)"

;"d10=d0-de"

;define delay echo 


\section{Appendix}

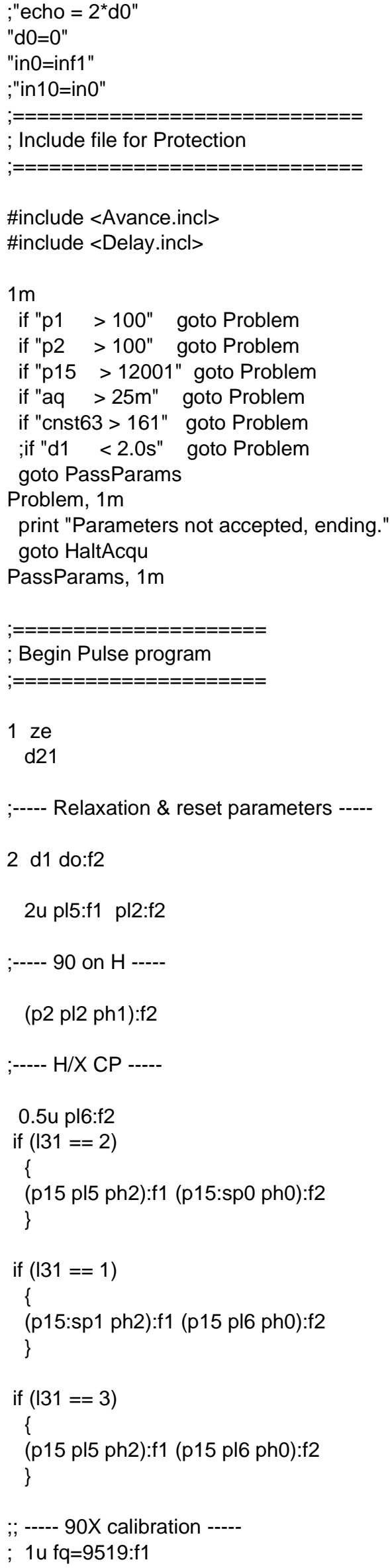

2 d1 do:f2

2u pl5:f1 pl2:f2 


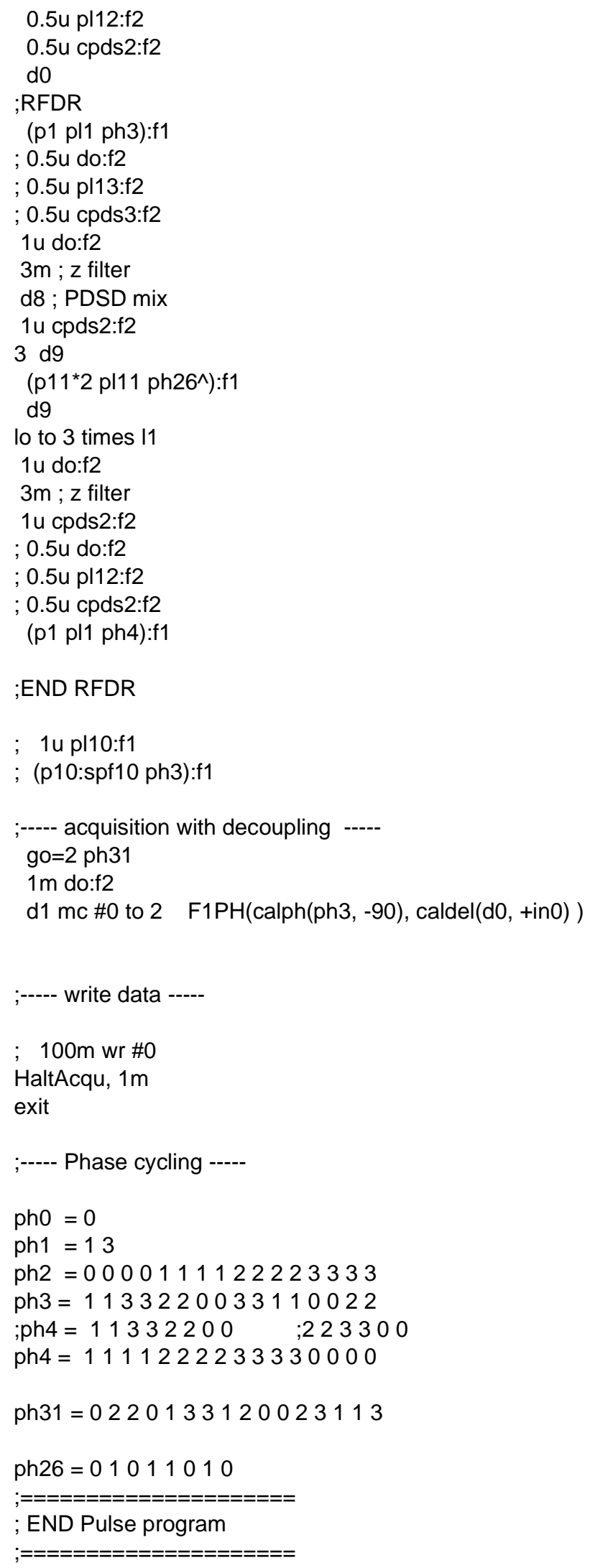




\section{Appendix}

\section{${ }^{15} N^{13} C$ TEDOR}

;Ztedor

;version: 2.0 TS3 / 7/14/2011

;transverse echo double resonance experiment

;Tested 12/06 in jos1206 on AV III console

;updated and tested JOS 07/14/2011

;Reference:

;C.P. Jaroniec, C. Filip, and R.G. Griffin, JACS 124, 2002, 10728-10742

;Avance III version

;parameters:

;d1 : recycle delay

;d31 : =1s/cnst31, 1 rotor period, to check correct cnst31 value

;p2 : X 180 degree pulse

;p3 : 90 degree $1 \mathrm{H}$ pulse at pl12

;p5 : 90 pulse on Y channel

;p12 : 180 deg. Pulse on the $Y$ channel

;p15 : contact pulse

;cnst31 : must be set to the spinning speed (in $\mathrm{Hz}$ ).

;spnam0 : file name for variable amplitude CP

;pl1 : to drive HP-X transmitter (CP power)

;pl3 : Y rf-power for pi pulse

;pl11 : X rf-power for pi pulse

;pl12 : 1H power for decoupling

;pl13 : 1H power for decoupling during recoupling (for instance using lgcw)

;pl14 : rf-power for z-filter use f_nutation= $f$ _rotation for recoupling of protons to $13 \mathrm{C}$ fast quenching of transverse magnetization

;sp0 : rf-power for proton contact pulse

;cpdprg2 : decoupling sequence for REDOR

;pcpd2 : decoupling pulse width

;cpdprg2 : decoupling during recoupling block us cw13 or lgcw

;FnMODE: use States-TPPI, States or TPPI

;ZGOPTNS : -DsmallSW for rotor synchronization for spectral width in F1 < sample rotation rate

;19 : fraction of rotation rate for 11 increment for choice of smallSW else multiple of rotor frequency for larger SW 
;ns : $=n^{\star} 16=n^{\star} 32$ with $t d=$ phase cycle

;CLASS=Solids

;\$DIM=2D

;\$TYPE=cross 117hird117sing117n

;\$SUBTYPE=TEDOR

;\$COMMENT=heteronuclear correlation

"d25=0.25s/cnst31-1u"

"d26=0.25s/cnst31-(p12/2)" ; one-quarter rotor cycle ...

"d27=0.25s/cnst31-(p1)"

"d28=0.25s/cnst31-5u"

“d29=0.75s/cnst31-(p2/2)"

"d31=1s/cnst31"

define pulse tauz

“tauz $=18^{*} 1 \mathrm{~s} / \mathrm{cnst} 31-\mathrm{p} 1 "$

\#include <trigg.incl>

"d0=1s/(19*cnst31)"

"acqt $0=1 u^{*}$ cnst $11 "$

define delay t1incr

"10=0"

\#ifdef smallsW

define delay tau

"tau=1s*|9/cnst31"

“d2=1s/cnst31-p5-p1"

"t1incr $=\left(1 s^{\star} \mid 9\right) /($ cnst31)"

"in $0=t 1$ incr"

"inf1=ino"

\#else

define delay tau

"tau=1s*(2*19-1)/(19*cnst31)"

" $\mid 10=1 "$

“|11=|10 \%19"

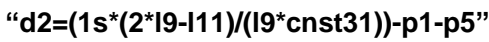




\title{
8 Appendix
}

\author{
"t1 incr=1s/(19*cnst31)" \\ "in0=t1incr" \\ “inf1=in0" \\ \#endif \\ define delay mix \\ “mix=4*2* $11 /$ cnst $31 "$ \\ $1 \mathrm{ze}$ \\ t1incr \\ tau \\ mix
}

d2

$230 \mathrm{~m}$

d1 do:f2

\#ifndef smallSW

"I11=|10 \% I9"

“d2=1s*(2*I9-I11)/(19*cnst31)"

\#endif

10u pl1:f1

;set power level to drive HP amplifier

10u pl12:f2

;set decoupler power

10u pl3:f3

\#include <p15_prot.incl>

;make sure p15 does not exceed $10 \mathrm{msec}$

;let supervisor change this pulseprogram if

;more is needed

\#ifndef lacq $\quad l^{\star}$ disable protection file for long acquisition change decoupling power !!! or you risk probe damage *I

$l^{*}$ if you set the label lacq (ZGOPTNS -Dlacq), the protection is disabled */

\#include <aq_prot.incl>

;allows max. 50 msec acquisition time, supervisor

;may change to max. $1 \mathrm{~s}$ at less than $5 \%$ duty cycle

;and reduced decoupling field

\#endif

1m rpp8
$1 \mathrm{~m}$ rpp9
$1 \mathrm{~m}$ rpp10
$1 \mathrm{~m}$ rpp11




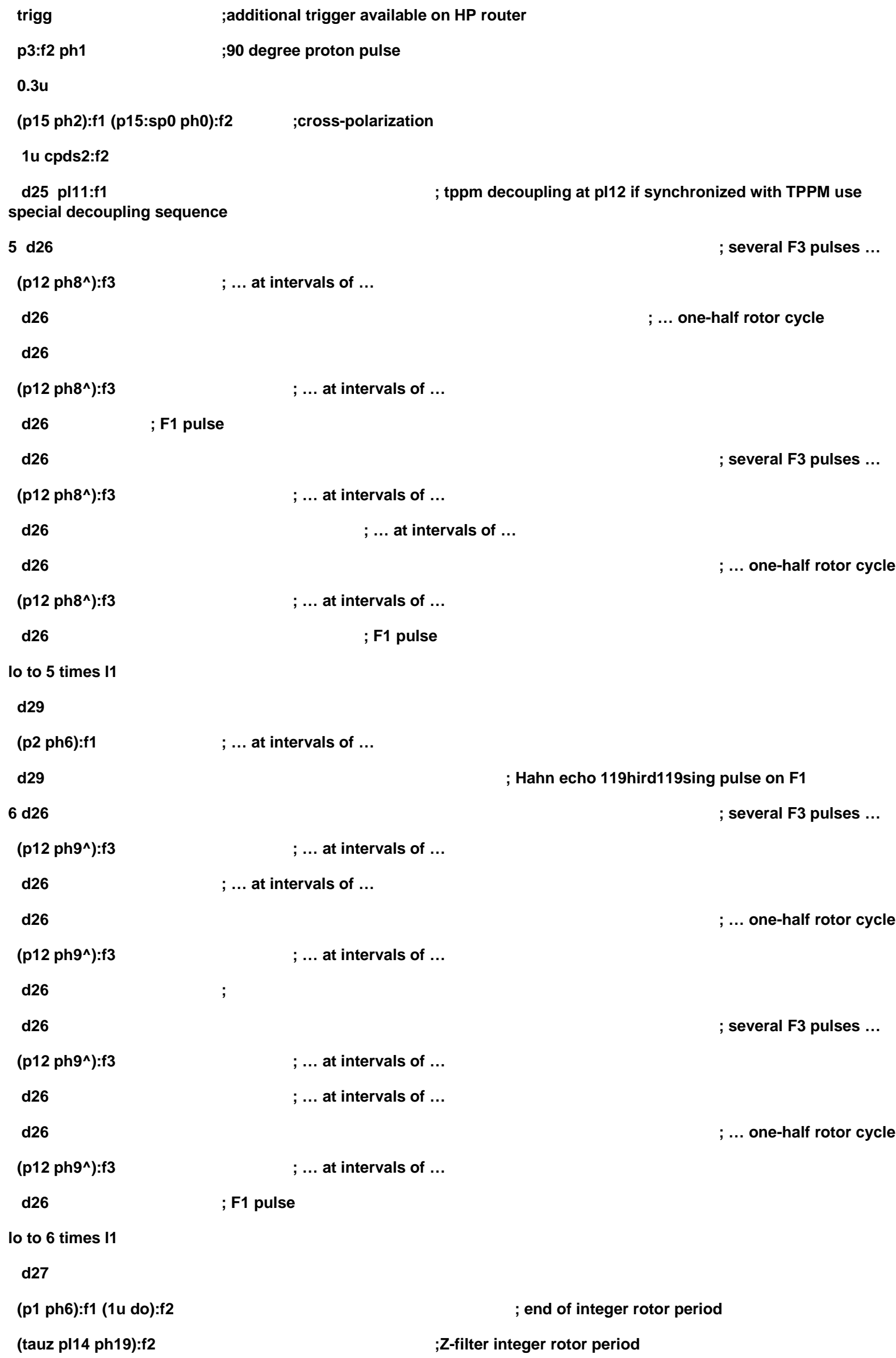

lo to 5 times I1

d29

(p2 ph6):f1

; $\ldots$ at intervals of ...

d29

; Hahn echo 119hird119sing pulse on F1

$6 \mathrm{~d} 26$ ; several F3 pulses ...

(p12 ph9^):f3 $; \ldots$ at intervals of ...

d26 ; ... at intervals of ...

d26 ; ... one-half rotor cycle

$(p 12$ ph9^):f3 , ... at intervals of ... d26 d26 ; several F3 pulses ...

(p12 ph9^):f3 $; \ldots$ at intervals of $\ldots$ d26 $; \ldots$ at intervals of ... d26 $; \ldots$ at intervals of ...

(p12 ph9^):f3 ; F1 pulse

d26

lo to 6 times 11

d27

(p1 ph6):f1 (1u do):f2 ; end of integer rotor period

(tauz pl14 ph19):f2 ;Z-filter integer rotor period 
8 Appendix

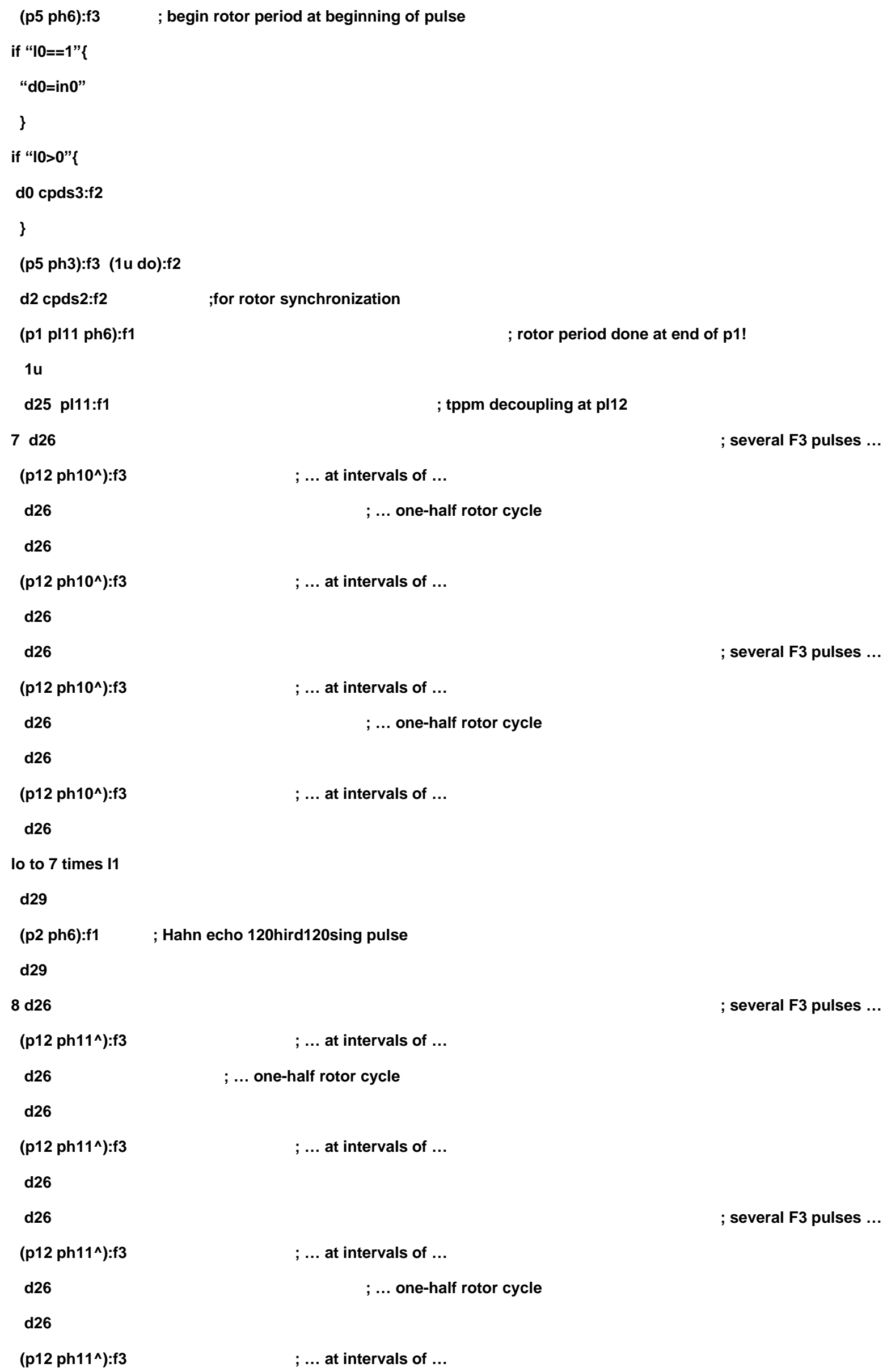


d26

lo to 8 times 11

d27

(p1 ph4):f1 (1u do):f2

(tauz pl14 ph19):f2

(p1 ph5):f1 (1u cpds3):f2

go=2 ph31

$1 \mathrm{~m}$ do:f2

$30 \mathrm{~m} \mathrm{mc} \# 0$ to $2 \mathrm{~F} 1 \mathrm{PH}(\mathrm{ip} 3$,id0 \& iu10 \& iu0)

HaltAcqu, 1m

exit

ph1 $=\{1\}^{\star} 16\{3\}^{\star} 16$

pho $=0$

ph2 $=0$

ph3 $=02$

ph4= 1133

ph5= 0000111122223333

ph6 $=0$

ph8= 0101101023233232

;xy16

ph9= 0101101023233232

ph10= 0101101023233232

$p h 11=0101101023233232$

ph19= 0

$p h 31=31130220$

13312002

13312002

31130220

;fixed inversion of $15 \mathrm{~N}$ dim -lba 6/2017

;XHHY.crmn NHHC-type experiment with Y/X decoupling during t1/t2 ;written by AL\&GP\&SL 9.12.2008

;modified by land from NHHC.ebm 6.2017

;p3 : proton 90 at power level pl2

;pl2 : proton $\mathrm{Pi} / 2$

\section{; Hahn echo occurs about now}




\section{Appendix}

;p15 : long contact time at pl1 (f3) and sp20 (f2)

;p16 : short contact time at pl1 (f3) and sp20 (f2)

;pl5 : X power level during contact

;sp20 : proton power level during first CP

;sp21 : proton power level during second CP

;p17 : short contact time at pl4 (f1) and sp0 (f2)

;pl4 : 122hird CP (Y power level during contact)

;sp0 : proton power level during third CP

;pl12 : decoupling power level (if not pl13)

;pl13 : special decoupling power level

;pl16 WALTZ 15N decoupling power

;pl1 13C power level for pulses

;pl3 15N hard pulse

;p7 15N hard pulse

;p2 13C Pi/2

;d8 : mixing time

;d1 : recycle delay

;d21 : z filter time (no 1H power)

;cnst21 : on resonance, usually $=0$

;pcpd2 : pulse length in decoupling sequence

;cpdprg2 : cw, tppm (at pl12), or Igs, cwlg. Cwlgs (LG-decoupling

;spnam0 : use e.g. ramp.100 for variable amplitude CP

;here pl13 is used instead of pl12)

\section{$\underline{\mathrm{hNHHC}}$}

\#include <Avancesolids.incl>

;\#ifdef fslg

;\#include <lgcalc.incl>

;cnst20 : RF field achieved at pl13

;cnst21 : on resonance, usually $=0$

;cnst22 : positive LG offset

;cnst23 : negative LG offset

;cnst24 : additional LG-offset

;\#endif $/{ }^{*}$ fslg */

;cnst11 : to adjust t=0 for acquisition, if digmod = baseopt 
"acqt $0=1 u^{*}$ cnst11"

"d0=0"

"in0=inf1"

1 ze

2 d1 do:f2

;1m do:f3

1u pl1:f1 pl2:f2 pl3:f3

\#include <p15_prot.incl>

;make sure p15 does not exceed $10 \mathrm{msec}$

;let supervisor change this pulseprogram if

;more is needed

;\#ifndef lacq

damage

;disable protection file for long acquisition change decoupling power !!! or you risk probe

;if you set the label lacq (ZGOPTNS -Dlacq), the protection is disabled

\#include <aq_prot.incl>

;allows max. 50 msec acquisition time, supervisor

;may change to max. $1 \mathrm{~s}$ at less than $5 \%$ duty cycle

;and reduced decoupling field

;\#endif

$1 \mathrm{u} f q=\mathrm{cnst} 21: \mathrm{f} 2$

(p3 ph1 pl2):f2 ;(ralign (p3 ph1 pl2):f2 (p7 ph13 pl3):f3)

(p15 ph2 pl5):f3 (p15:sp20 ph10):f2 ;1H to 15N long contact pulse with square or ramp

(p7 ph11 pl3):f3

;add shaped 180 pulse for selective seq.

d21 ;z filter

1u cpds2:f2

(p7 ph12 pl3):f3

;(center (d0) (p2² pl1 ph0):f1) ;13C refocusing pulse during t1

do

$0.5 u$ do:f2

(p16 pl5 ph3):f3 (p16:sp21 ph4):f2 ;15N to 1H short contact pulse with square or ramp

(p3 ph5 pl2):f2 ;1H flip-back pulse

d8 
8 Appendix

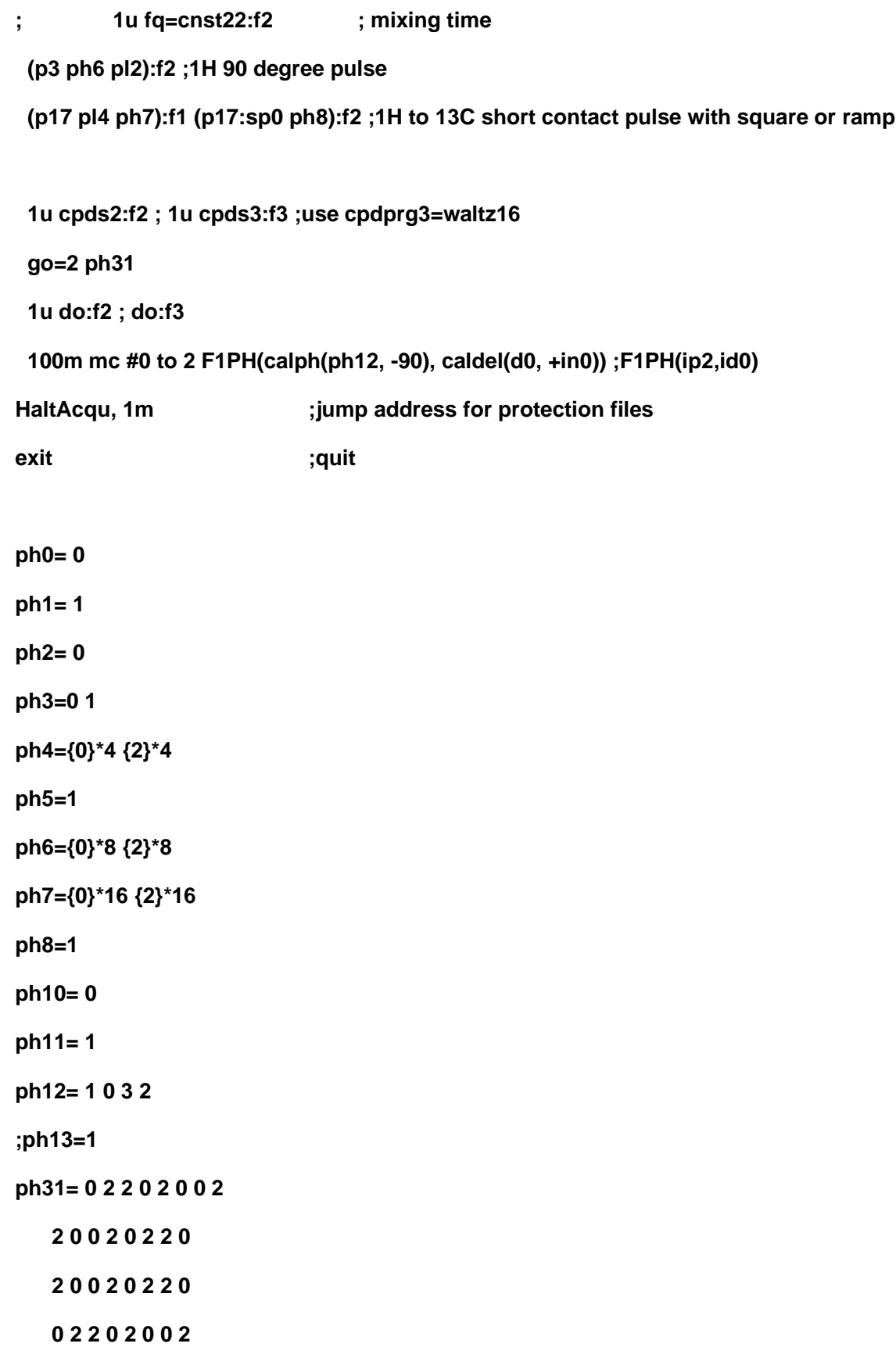

\section{HcaCBcaNH}

; 3D $(\mathrm{H})(\mathrm{CA}) \mathrm{CB}(\mathrm{CA}) \mathrm{NH}$ developed at $\mathrm{CRMN}$ in the group of G. Pintacuda and modified at the MPIBPC ; Recent notes:

; this version does not require $13 \mathrm{C}$ axis inversion

;Avance III version ;parameters:

;p1: $1 \mathrm{H} 90$ pulse duration

;p3 : 13C 90 pulse duration 
;p7 : 15N 90 pulse duration

;p30 : water suppression time (30-100 ms)

;cnst20 : CB offset in ppm (39.7)

;cnst21 : CO offset in ppm (173.7)

;cnst22 : CA offset in ppm (53.7)

;cnst23 : CO/CO offset in ppm (113.7)

;d1 : recycle delay

;d0 : 15N initial evolution time

;d10 : 1/2 of $13 \mathrm{CB}$ initial evolution time

;in0 : increment for $15 \mathrm{~N}$ evolution

;in10 : 1/2 increment for 13CA evolution

;cpdprg1 : tppm (at pl13) or waltz (at pl13)

;cpdprg4 : cwY (at pl12)

;cpdprg5 : cwX (at pl12)

;pcpd1 : pulse length in decoupling sequence (2xtau_r for tppm, 25us for $10 \mathrm{kHz}$ waltz)

;pl1 : power level of $1 \mathrm{H}$ hard pulse

;pl12 : decoupling $(10-15 \mathrm{kHz})$

;pl13 : water suppression

;spnam1 : $1 \mathrm{H}$ shape for $1 \mathrm{H}->13 \mathrm{CA}$ CP (ramp 10-20\%)

;spoal1 : N/A

;spoff1 : [ON/RES]

;sp1 : $1 \mathrm{H}$ power level during $1 \mathrm{H}->13 \mathrm{CA} \mathrm{CP}$

;spnam10 : $1 \mathrm{H}$ shape for $15 \mathrm{~N}->1 \mathrm{H}(\mathrm{N}) \mathrm{CP}$ (ramp 10-20\%)

;spoal10 : N/A

;spoff10 : [ON/RES]

;sp10 : $1 \mathrm{H}$ power level during $15 \mathrm{~N}->1 \mathrm{H} \mathrm{CP}$

;cpdprg2 : 15N decoupling pattern during acq (waltz-16)

;p17 : contact time 15N->1H(N) CP (300-500 us)

;pcpd2 : pulse length in $15 \mathrm{~N}$ decoupling sequence (25 us)

;pl7 : power level for $15 \mathrm{~N}$ hard pulse

;pl16 : power level for $15 \mathrm{~N}$ decoupling (corr. to $10 \mathrm{kHz}$ )

;pl2 : power level for $15 \mathrm{~N}$ hard pulse

;pl20 : 15N power level for $15 \mathrm{~N}->1 \mathrm{H} \mathrm{CP}$

;spnam2 : 15N shape for 13CA->15N CP (tan-c100-w10pct)

;sp2 : 15N power level for 13CA->15N CP

;spoal2 : N/A

;spoff2 : [ON/RES]

;p15 : contact time $1 \mathrm{H}->13 \mathrm{CA}$ CP $(5 \mathrm{~ms})$

;p16 : contact time 13CA->15N CP (10 ms)

;p19 : Q3 CA-CB pulse duration

;pcpd3 : pulse length in 13C decoupling sequence (25 us)

;cpdprg3 : 13CO/CA decoupling pattern during 15N evol (waltz-16)

;pl3 : power level of 13C hard pulse [REFERENCE]

;pl11 : power level of 13C hard pulse [REFERENCE]

;pl17 : power level for 13CO/CA decoupling $(10 \mathrm{kHz})$

;spnam9 : 13C shape for 13CA->15N CP (rectangle)

;sp9 : 13C power for 13CA->15N CP

;spoal9 : 0 (align at the beginning)

;spoff9 : offset from CB to CA

;spnam19:13CA+CB selective pulse shape (Q3)

;spoal19: N/A

;spoff19 : [ON/RES CA-CB]

;spnam29 : 13C shape for $1 \mathrm{H}->13 \mathrm{CA} \mathrm{CP}$ (rectangle)

;sp29 : 13C power level for $1 \mathrm{H}->13 \mathrm{CA}$ CP

;d28 : Half delay for the CA-CB transfer (max $7 \mathrm{~ms}$ )

;cnst10 : estimated bulk T2 of CA [sec]

;spoal29:10 (align at the end)

;spoff29 : offset from CB to CA

;zgoptns : -Dfslg, -Dlacq, or blank

;\$COMMENT=Inverse Cp with INEPT CBCA mixing

;\$CLASS=Solids 


\section{Appendix}

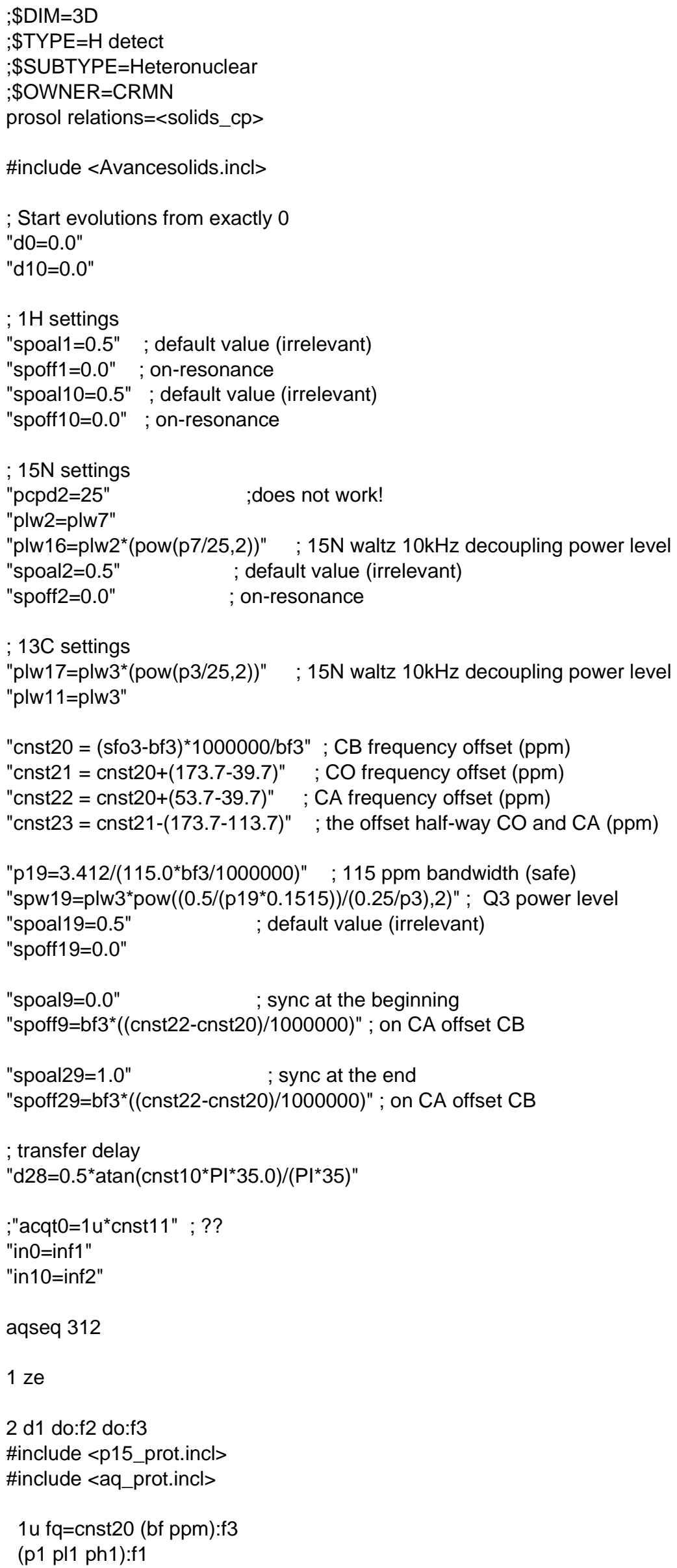




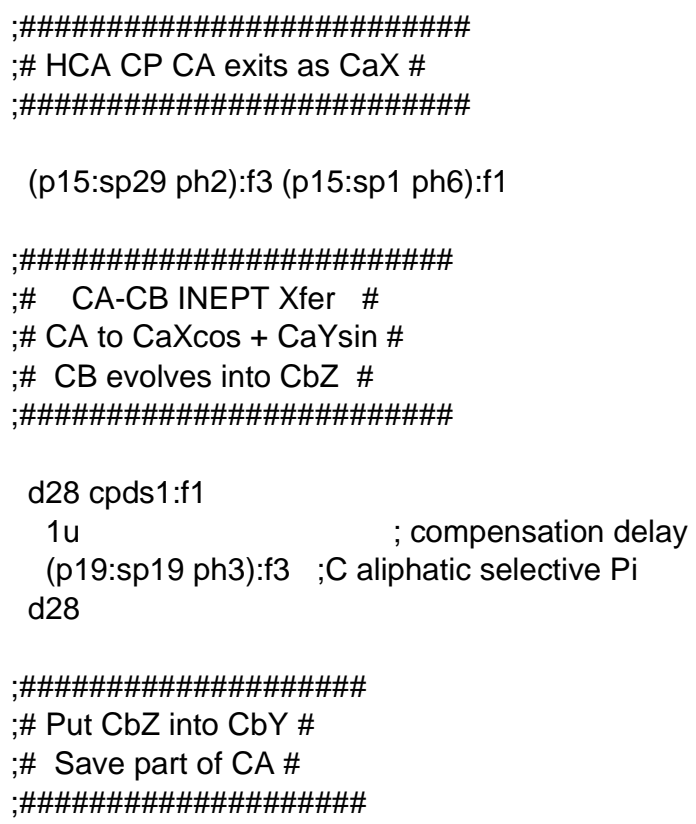

1u cpds2:f2

; one does not need $15 \mathrm{~N}$ decoupling for $13 \mathrm{CB}$ evolution

; however (!) there is no way to eliminate CA signal (when d28 is shorter than for a full transfer)

; and CA peaks appear in the spectrum with the opposite phase

; then anyway $15 \mathrm{~N}$ decoupling is useful to narrow their lines (to decrease overlap)

(p3 pl11 ph4):f3

;\# Time Evolution (CB and the remaining CA)

d10

;\#\#\#\#\#\#\#\#\#\#\#\#\#\#\#

;\# Put them back \#

;\#\#\#\#\#\#\#\#\#\#\#\#\#\#\#

(p3 pl11 ph5):f3 ;C ali transverse selective 90

$1 \mathrm{u}$ do:f2

d28

(p19:sp19 ph20):f3 ;C aliphatic selective Pi d28

1u do:f1

;\#\#\#\#\#\#\#\#

; CN CP \#

;\#\#\#\#\#\#\#\#

; (p3 pl11 ph6):f3

d8 do:f1

; (p3 pl11 ph6):f3

iu

(p16:sp9 ph7):f3 (p16:sp2 ph8):f2

$1 \mathrm{ufq}=\mathrm{cnst} 23$ (bf ppm):f3; go back to in between of $\mathrm{CO}$ and $\mathrm{CA}$ for efficient decoupling

1u cpds1:f1 cpds3:f3

d0

1u do:f1 do:f3 


\section{Appendix}

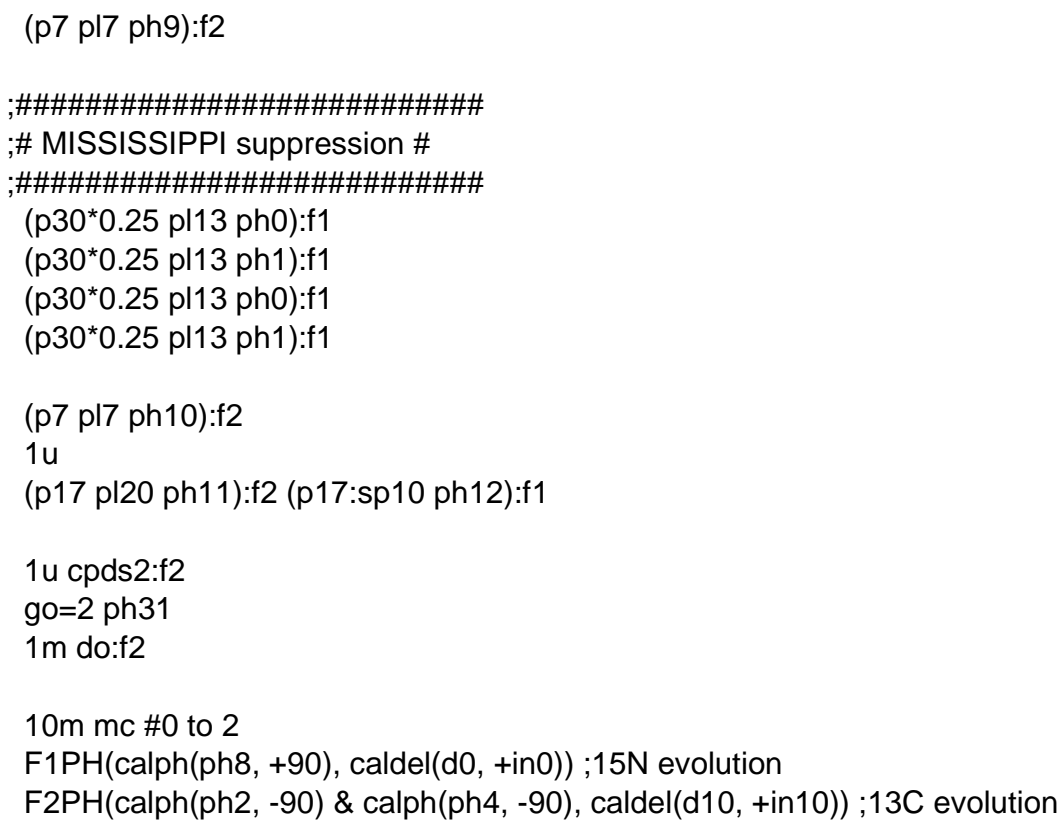

\section{hCONH}

; 3D $(\mathrm{H}) \mathrm{CONH}$ developed at CRMN in the group of G. Pintacuda

; Recent notes:

; this version does not require $13 \mathrm{C}$ axis inversion

;Avance III version ;parameters:

;p1 : $1 \mathrm{H} 90$ pulse duration

;p3 : 13C 90 pulse duration

;p7 : 15N 90 pulse duration

;d30 : water suppression time (30-100 ms)

;cnst21 : CO offset in ppm (173.7)

;cnst22 : CA offset in ppm (53.7) 
;cnst23 : CO/CO offset in ppm (113.7)

$; \mathrm{d} 1$ : recycle delay

$; \mathrm{d} 0 \quad: 15 \mathrm{~N}$ initial evolution time

;d10 : 1/2 of $13 \mathrm{CO}$ initial evolution time

;in0 : increment for $15 \mathrm{~N}$ evolution

;in10 : 1/2 increment for $13 \mathrm{CO}$ evolution

;cpdprg1 : tppm (at pl13) or waltz (at pl13)

;cpdprg4 : cwY (at pl12)

;cpdprg5 : cwX (at pl12)

;pcpd1 : pulse length in decoupling sequence (2xtau_r for tppm, 25us for $10 \mathrm{kHz}$ waltz)

;pl1 : power level of $1 \mathrm{H}$ hard pulse

;pl12 : power level for $1 \mathrm{H}$ decoupling (usually swTPPM at MAS/4 -1 or $-2 \mathrm{kHz}$ )

;pl13 : power level of MISSISSIPI water suppression (10-15 kHz)

;spnam1 : $1 \mathrm{H}$ shape for $1 \mathrm{H}->13 \mathrm{CO} \mathrm{CP}$ (ramp 10-20\%)

;spoal1 : N/A

;spoff1 : [ON/RES]

;sp1 : $1 \mathrm{H}$ power level during $1 \mathrm{H}->13 \mathrm{CO} \mathrm{CP}$

;spnam10 : $1 \mathrm{H}$ shape for $15 \mathrm{~N}->1 \mathrm{H}(\mathrm{N}) \mathrm{CP}$ (ramp 10-20\%)

;spoal10 : N/A

;spoff10 : [ON/RES]

;sp10 : 1H power level during 15N->1H CP

;cpdprg2 : 15N decoupling pattern during acq (waltz-16)

;p17 : contact time 15N->1H(N) CP (300-500 us)

;pcpd2 : pulse length in $15 \mathrm{~N}$ decoupling sequence (25 us)

;pl7 : power level for $15 \mathrm{~N}$ hard pulse

;pl16 : power level for $15 \mathrm{~N}$ decoupling (corr. to $10 \mathrm{kHz}$ )

;pl2 : power level for $15 \mathrm{~N}$ hard pulse

;pl20 : 15N power level for $15 \mathrm{~N}->1 \mathrm{H} \mathrm{CP}$

;spnam2 : $15 \mathrm{~N}$ shape for 13CO->15N CP (tan-c100-w10pct)

;sp2 : 15N power level for 13CO->15N CP

;spoal2 : N/A

;spoff2 : [ON/RES]

;p15 : contact time $1 \mathrm{H}->13 \mathrm{CO} \mathrm{CP}(5 \mathrm{~ms})$

;p16 : contact time 13CO->15N CP (10 ms)

;p18 : Q3 CO pulse duration

;p19 : Q3 CA pulse duration

;p30 : water suppression duration

;pcpd3 : pulse length in 13C decoupling sequence (25 us)

;cpdprg3 : 13CO/CA decoupling pattern during 15N evol (waltz-16)

;pl3 : power level of 13C hard pulse [REFERENCE]

;pl17 : power level for 13CO/CA decoupling (10 kHz)

;spnam8 : 13C shape for 13CO->15N CP (rectangle)

;sp8 : 13C power for 13CO->15N CP

;spoal8 : N/A

;spoff8 : [ON/RES CO]

;spnam18 : 13CO selective pulse shape (Q3)

;spoal18: N/A

;spoff18 : [ON/RES CO]

;spnam19 : 13CA selective pulse shape (Q3)

;spoal19: N/A

;spoff19 : [ON/RES CA]

;spnam28 : 13C shape for $1 \mathrm{H}->13 \mathrm{CO} \mathrm{CP}$ (rectangle)

;sp28 : 13C power level for $1 \mathrm{H}->13 \mathrm{CO} \mathrm{CP}$

;spoal28 : N/A

;spoff28 : [ON/RES CO]

;zgoptns : -Dfslg, -Dlacq, or blank

;\$COMMENT=Inverse Cp with INEPT CBCA mixing

; $\$$ CLASS=Solids

;\$DIM=3D

;\$YPE=H detect 


\section{Appendix}

;\$SUBTYPE=Heteronuclea ;\$OWNER=CRMN

\#include <Avancesolids.incl>

; Start evolutions from exactly 0

"d0=0.0"

"d10=0.0"

; $1 \mathrm{H}$ settings

"spoal1=0.5" ; default value (irrelevant)

"spoff1=0.0" ; on-resonance

"spoal10=0.5" ; default value (irrelevant)

"spoff10=0.0" ; on-resonance

; $15 \mathrm{~N}$ settings

"pcpd2=25"

;does not work

"plw2=plw7"

"plw16=plw2*(pow(p7/25,2))" ; 15N waltz 10kHz decoupling power level

"spoal $2=0.5 "$; default value (irrelevant)

"spoff2=0.0" ; on-resonance

; 13C settings

"plw17=plw3*(pow(p3/25,2))" ; 15N waltz 10kHz decoupling power level

"cnst21 = (sfo3-bf3)*1000000/bf3" ;CO frequency offset (ppm)

"cnst22 = cnst21-(173.7-53.7)" ; CA frequency offset $(\mathrm{ppm})$

"cnst23 = cnst21-(173.7-113.7)" ; the offset half-way CO and CA (ppm)

"p18=3.412/(95.0*bf3/1000000)" ; 95 ppm bandwidth (safe)

"spw18=plw3*pow $((0.5 /($ p18*0.1515) $) /(0.25 / p 3), 2) "$; Q3 power level

"spoal18=0.5" ; default value (irrelevant)

"spoff $18=0.0$ "

"p19=3.412/(105.0*bf3/1000000)" ; 105 ppm bandwidth (safe)

"spw19=plw3*pow $((0.5 /($ p19*0.1515) $) /(0.25 /$ p3 $), 2) "$; Q3 power level

"spoal19=0.5" ; default value (irrelevant)

"spoff19=bf3* $(($ cnst22-cnst21)/1000000)" ; CA frequency

"spoal8=0.5"

"spoff8=0.0"

; default value (irrelevant)

; on-resonance

"spoal28=0.5"

"spoff28=0.0"

; default value (irrelevant)

; on-resonance

;"acqt $0=1 \mathrm{u}^{*} \mathrm{cnst} 11$ " ??

"in0=inf1"

"in10=inf2/2"

aqseq 312

;aqseq 321

$1 \mathrm{ze}$

2 d1 do:f2 do:f3

\#include <p15_prot.incl>

\#include <aq_prot.incl>

$1 \mathrm{u} \mathrm{fq}=\mathrm{cnst} 21(\mathrm{bf} \mathrm{ppm}): \mathrm{f3} \quad$;go back to the CO frequency

(p1 pl1 ph3):f1

(p15:sp28 ph15):f3 (p15:sp1 ph20):f1

1u cpds $1: f 1$ 
(p19:sp19 ph19):f3 ;CA selective Pi

$1 \mathrm{u}$

(p18:sp18 ph18):f3 ;CO selective Pi

$1 \mathrm{u}$

d10

(center (p7² ph0 pl7):f2 (p19:sp19 ph19):f3) ;CA selective Pi

d10

1u do:f1

(p16:sp8 ph10):f3 (p16:sp2 ph2):f2

$1 \mathrm{u} f \mathrm{q}=\mathrm{cnst} 23(\mathrm{bf} \mathrm{ppm}): \mathrm{f3}$

1u cpds $1: f 1$ cpds3:f3

d0

1u do:f1 do:f3

(p7 pl7 ph5):f2

(p30*0.25 pl13 ph0):f1

(p30*0.25 pl13 ph1):f1

(p30*0.25 pl13 ph0):f1

(p30*0.25 pl13 ph1):f1

(p7 pl7 ph6):f2

(p17 pl20 ph7):f2 (p17:sp10 ph11):f1

1u cpds2:f2

$\mathrm{go}=2 \mathrm{ph} 31$

$1 \mathrm{~m}$ do:f2

$10 \mathrm{~m} \mathrm{mc} \# 0$ to 2

$\mathrm{F} 1 \mathrm{PH}(\mathrm{calph}(\mathrm{ph} 2,+90)$, caldel $(\mathrm{d} 0,+\mathrm{in} 0)) ; 15 \mathrm{~N}$ evolution

$\mathrm{F} 2 \mathrm{PH}(\mathrm{calph}(\mathrm{ph} 15,-90)$, caldel(d10, +in10)) ; $; 3 \mathrm{C}$ evolution

HaltAcqu, 1m ;jump address for protection files

exit ;quit

ph0 $=0$

ph1 $=1$

ph3 $=0$

ph20 = 11333311

ph15 $=0$

ph10 $=02$

ph2 = $11331133 ; 15 \mathrm{~N} \mathrm{CP}(15 \mathrm{~N}$ pulse $)$

ph5 $=0$

ph6 $=0$

ph18 = 0

ph19 $=0$

ph7 $=1$

ph11 = 1

ph31 = 13133131 


\section{hcaCBcacoNH}

; 3D $(\mathrm{H})(\mathrm{CA}) \mathrm{CB}(\mathrm{CA})(\mathrm{CO}) \mathrm{NH}$ developed at $\mathrm{CRMN}$ in the group of $\mathrm{G}$. Pintacuda

; Recent notes:

; this version does not require $13 \mathrm{C}$ axis inversion

;Avance III version

;parameters:

;p1 : $1 \mathrm{H} 90$ pulse duration

;p3 : 13C 90 pulse duration

;p7 : 15N 90 pulse duration

;d30 : water suppression time (30-100 ms)

;cnst20 : CB offset in ppm (37.7)

;cnst21: CO offset in ppm (173.7)

;cnst22 : CA offset in ppm (53.7)

;cnst23 : CO/CO offset in ppm (113.7)

$; \mathrm{d} 1$ : recycle delay

;d0 : $15 \mathrm{~N}$ initial evolution time

;d10 : 1/2 of 13CB initial evolution time

;in0 : increment for $15 \mathrm{~N}$ evolution

;in10 : 1/2 increment for 13CA evolution

;cpdprg1 : tppm (at pl13) or waltz (at pl13)

;cpdprg4 : cwY (at pl12)

;cpdprg5 : cwX (at pl12)

;pcpd1 : pulse length in decoupling sequence (2xtau_r for tppm, 25us for $10 \mathrm{kHz}$ waltz)

;pl1 : power level of $1 \mathrm{H}$ hard pulse

;pl12 : power tppm or waltz decoupling

;pl13 : power level water suppression

;spnam1 : $1 \mathrm{H}$ shape for $1 \mathrm{H}->13 \mathrm{CA}$ CP (ramp 10-20\%)

;spoal1 : N/A

;spoffs $1:$ [ON/RES]

;sp1 : $1 \mathrm{H}$ power level during $1 \mathrm{H}->13 \mathrm{CA} \mathrm{CP}$

;spnam10 : $1 \mathrm{H}$ shape for $15 \mathrm{~N}->1 \mathrm{H}(\mathrm{N}) \mathrm{CP}$ (ramp 10-20\%)

;spoal10 : N/A

;spoffs $10:$ [ON/RES]

;sp10 : $1 \mathrm{H}$ power level during $15 \mathrm{~N}->1 \mathrm{H} \mathrm{CP}$

;cpdprg2 : 15N decoupling pattern during acq (waltz-16)

;p17 : contact time $15 \mathrm{~N}->1 \mathrm{H}(\mathrm{N}) \mathrm{CP}$ (300-500 us)

;pcpd2 : pulse length in $15 \mathrm{~N}$ decoupling sequence (25 us)

;pl7 : power level for $15 \mathrm{~N}$ hard pulse

;pl16 : power level for $15 \mathrm{~N}$ decoupling (corr. to $10 \mathrm{kHz}$ )

;pl2 : power level for $15 \mathrm{~N}$ hard pulse

;pl20 : 15N power level for $15 \mathrm{~N}->1 \mathrm{H} \mathrm{CP}$

;spnam2 : 15N shape for 13CO->15N CP (tan-c100-w10pct)

;sp2 : 15N power level for 13CO->15N CP

;spoal2 : N/A

;spoffs2 : [ON/RES]

;p15 : contact time $1 \mathrm{H}->13 \mathrm{CA} \mathrm{CP}(5 \mathrm{~ms})$

;p16 : contact time 13CO->15N CP (10 ms)

;pcpd3 : pulse length in 13C decoupling sequence (25 us)

;cpdprg3 : 13CO/CA decoupling pattern during 15N evol (waltz-16)

;pl3 : power level of $13 \mathrm{C}$ hard pulse [REFERENCE]

;plw11 : power level of 13C hard pulse [REFERENCE]

;plw17 : power level for 13CO/CA decoupling $(10 \mathrm{kHz})$

;spnam8 : 13C shape for 13CO->15N CP (rectangle)

;sp8 : 13C power for 13CO->15N CP

;spoal8 : N/A

;spoffs8 : [ON/RES]

;spnam29: 13C shape for $1 \mathrm{H}->13 \mathrm{CA}$ CP (rectangle)

;sp29 : 13C power level for $1 \mathrm{H}->13 \mathrm{CA} \mathrm{CP}$

;spoal29: 1 (align at the end) 


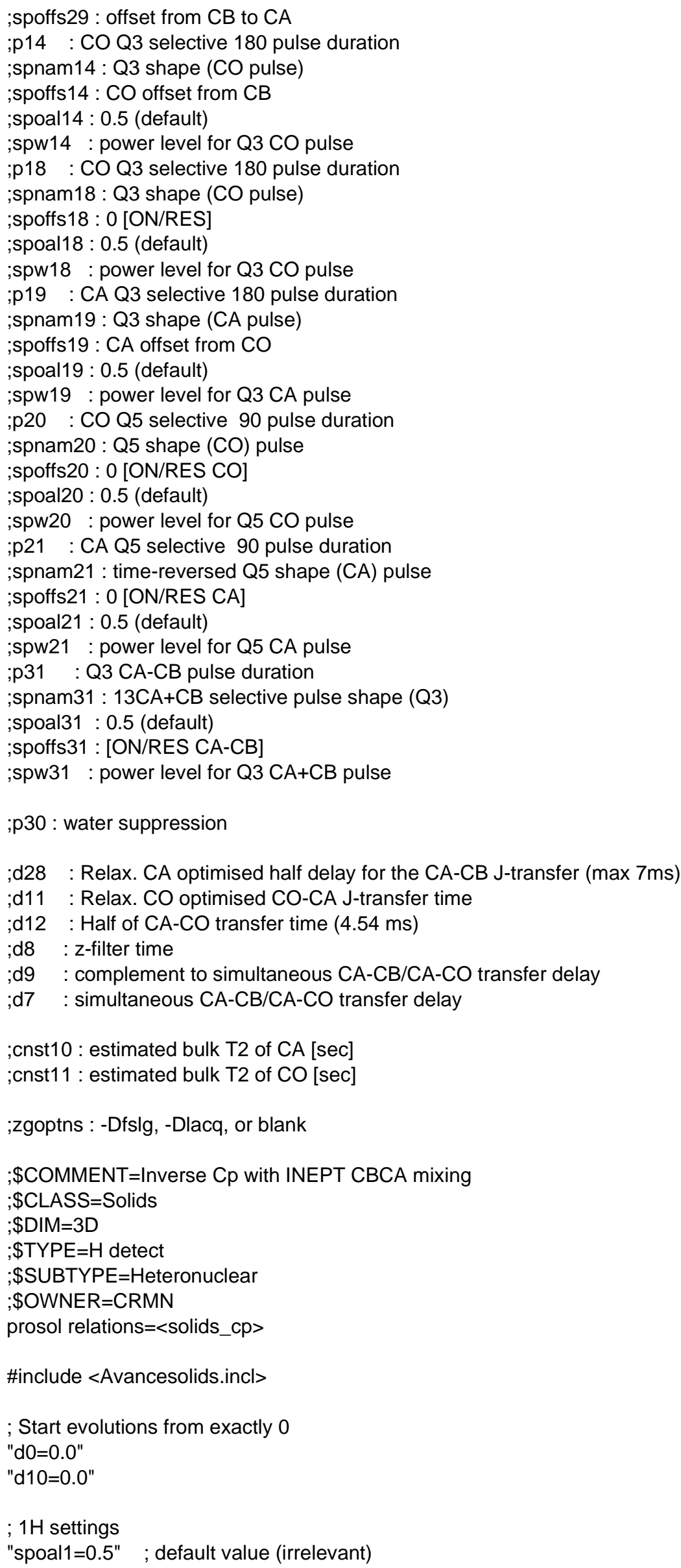




\section{Appendix}

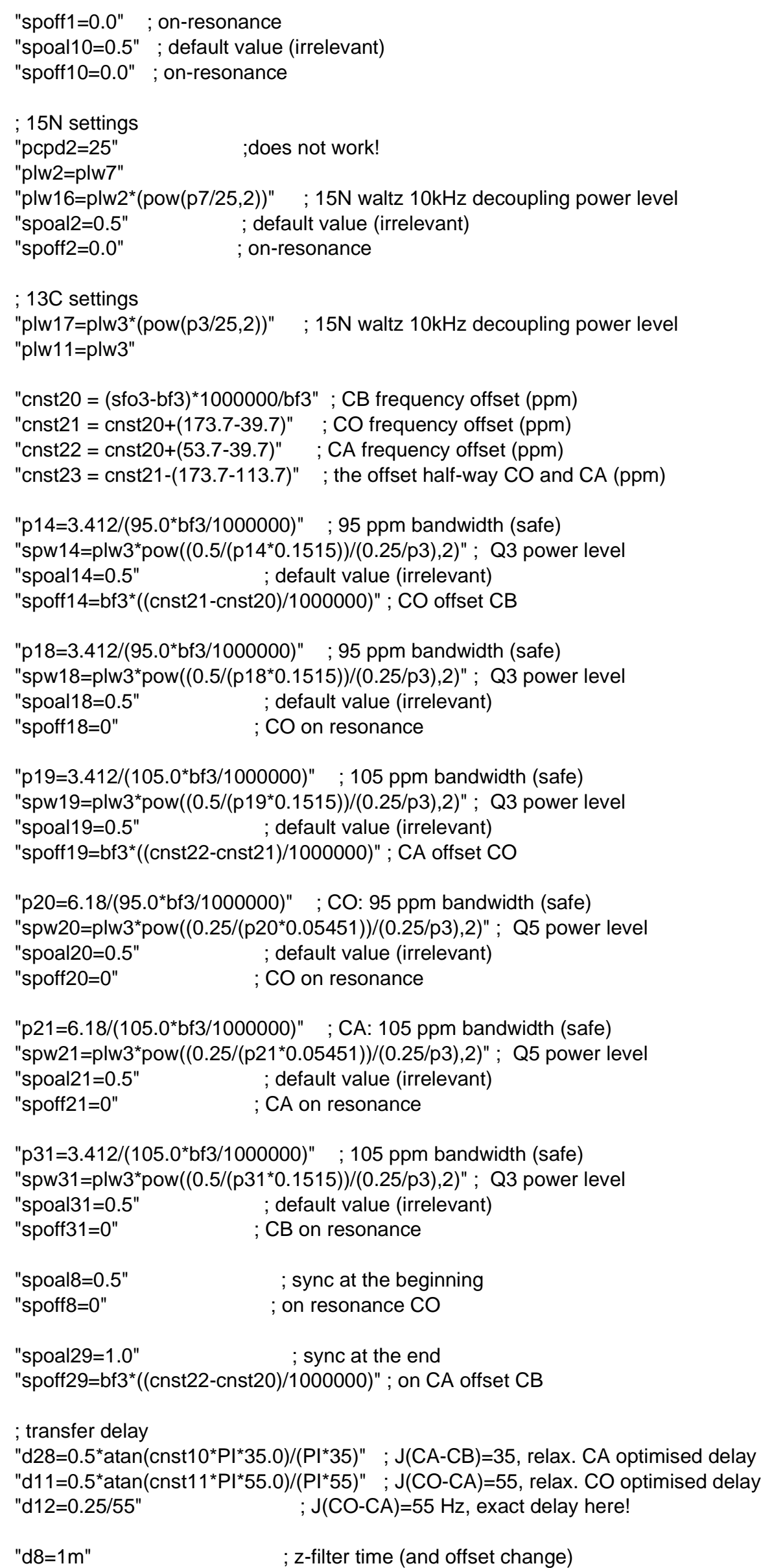


"d7=larger(d12+p18,d28)-p18"

"d9=larger(d12+p18,d28)-p18-d12"

"acqt $0=0 "$

"in0=inf1"

"in30=inf1"

"in10=inf2"

aqseq 312

1 ze

2 d1 do:f2 do:f3

\#include <p15_prot.incl $>$

\#include <aq_prot.incl>

$1 \mathrm{u} f q=\mathrm{cnst} 20(\mathrm{bf} p \mathrm{pm}): \mathrm{f3}$

(p1 pl1 ph1):f1

;\#\#\#\#\#\#\#\#\#\#\#\#\#\#\#\#\#\#\#\#\#\#

;\# HCA CP CA exits as CaX \#

;\#\#\#\#\#\#\#\#\#\#\#\#\#\#\#\#\#\#\#\#\#

(p15:sp29 ph2):f3 (p15:sp1 ph13):f1

;\#\#\#\#\#\#\#\#\#\#\#\#\#\#\#\#\#\#\#\#\#

;\# CA-CB INEPT Xfer \#

;\# CA to CaXcos + CaYsin \#

;\# CB evolves into CbZ \#

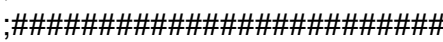

d28 cpds $1: f 1$

$1 \mathrm{u}$

(p31:sp31 ph3):f3 ;C aliphatic selective Pi

d28

;\#\#\#\#\#\#\#\#\#\#\#\#\#\#\#\#\#

;\# Put CbZ into CbY \#

;\# Save part of CA \#

;\#\#\#\#\#\#\#\#\#\#\#\#\#\#\#\#

1u cpds2:f2

(p3 pl11 ph4):f3 ；C ali transverse nonselective 90

;\# Time Evolution (CB and the rest of $\mathrm{CA}$ )

d10

;\#\#\#\#\#\#\#\#\#\#\#\#\#\#\#

;\# Put them back \#

;\#\#\#\#\#\#\#\#\#\#\#\#\#\#\#

(p3 pl11 ph5):f3 (1u do):f2 ～; C ali transverse nonselective 90

(p14:sp14 ph14):f3 ; CO selective Pi

d7

$(p 31: s p 31$ ph30):f3 $\quad ; C$ aliphatic selective $\mathrm{Pi}$
; simultaneous CA-CB/CA-CO transfer delay ; simultaneous CA-CB/CA-CO transfer delay 


\section{Appendix}

d9

(p14:sp14 ph14):f3 ; CO selective Pi

$\mathrm{d} 12$

(p21:sp21 ph21):f3 ; CA selective $\mathrm{Pi} / 2$

$\mathrm{d} 8 \mathrm{fq}=\mathrm{cnst} 21$ (bf ppm):f3 ; move offset from CA to CO

(p20:sp20 ph20):f3 ; CO selective $\mathrm{Pi} / 2$

;;; CO-CA J-evolution

$1 \mathrm{u}$

d11 ;first tau evolution period

(p19:sp19 ph19):f3 ;CA selective Pi

$2 \mathrm{u}$

(p18:sp18 ph18):f3 ;CO selective Pi

d11 ;second tau evolution period

(p19:sp19 ph19):f3 ;CA selective Pi

3u do:f1

;\#\#\#\#\#\#\#\#\#
;\# CN CP \#
;\#\#\#\#\#\#\#\#\#

(p16:sp8 ph7):f3 (p16:sp2 ph8):f2

$1 \mathrm{ufq}=\mathrm{cnst} 23(\mathrm{bf} p \mathrm{pm}): \mathrm{f} 3$; go back to in between of $\mathrm{CO}$ and $\mathrm{CA}$ for efficient decoupling $1 \mathrm{u}$ cpds $1: \mathrm{f} 1 \mathrm{cpds} 3: \mathrm{f3}$

d0

$1 \mathrm{u}$ do:f1 do:f3

(p7 pl7 ph9):f2

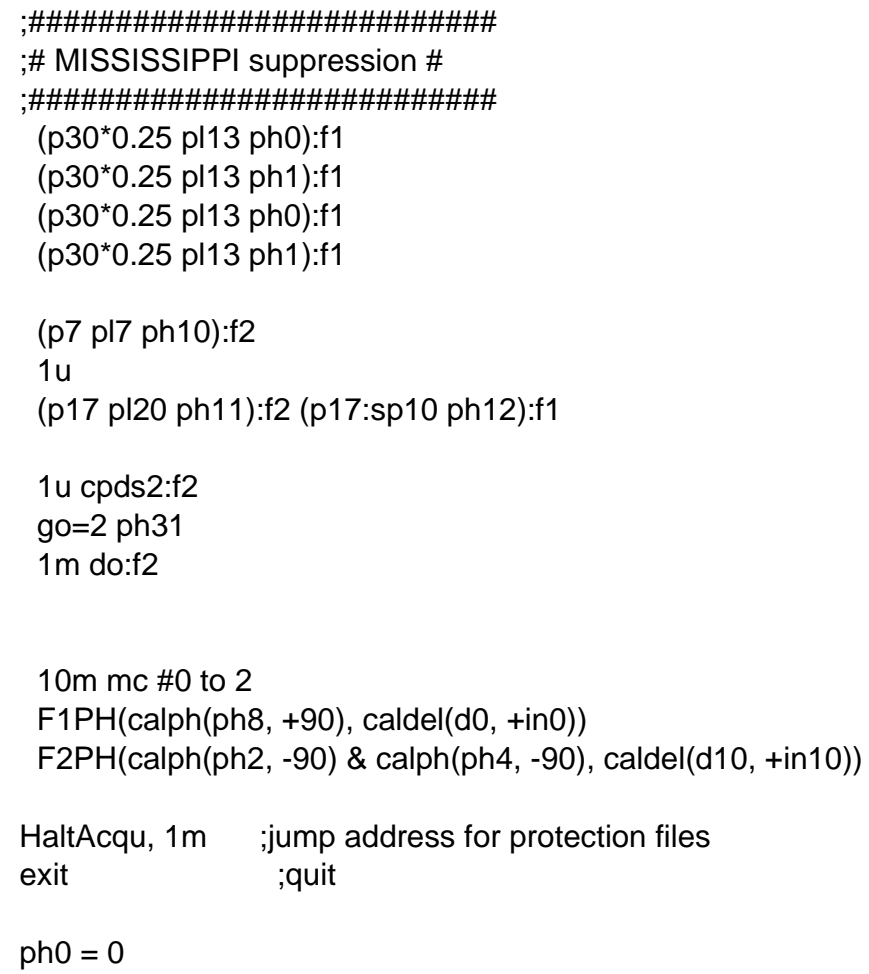




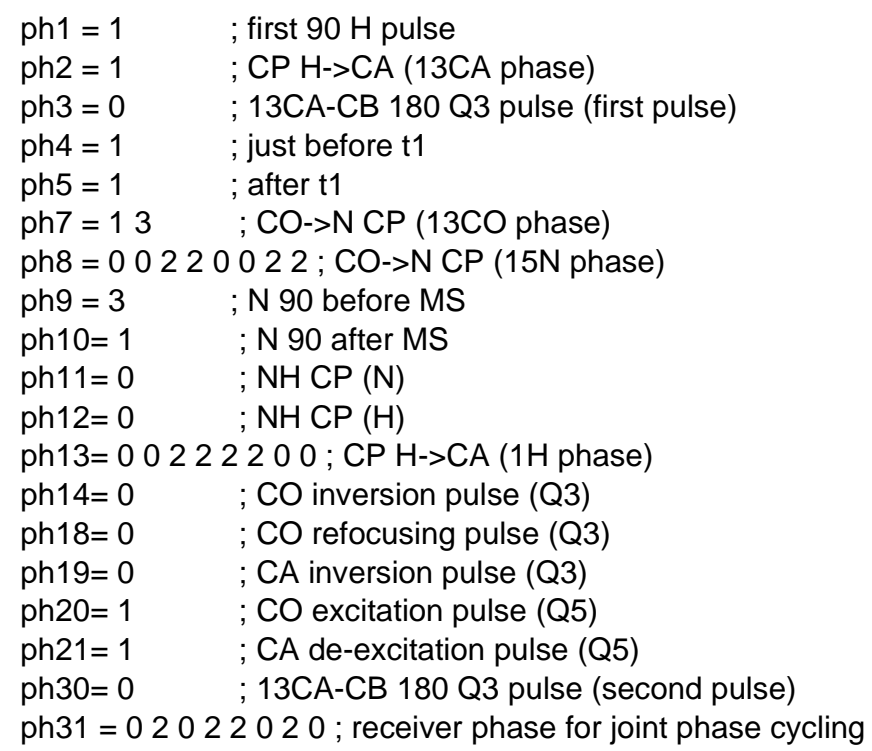

\title{
hCANH
}

; 3D (H)CANH developed at CRMN in the group of G. Pintacuda and modified at the MPIBPC

\author{
; Recent notes: \\ ; this version does not require $13 \mathrm{C}$ axis inversion
}

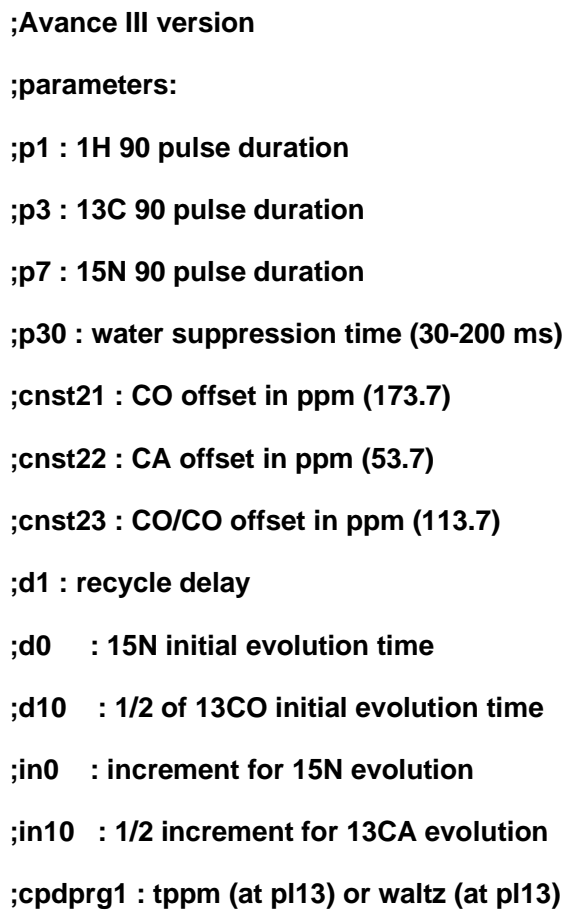




\section{Appendix}

;cpdprg4 : cwY (at pl12)

;cpdprg5 : cwX (at pl12)

;pcpd1 : pulse length in decoupling sequence (2xtau_r for tppm, 25us for 10kHz waltz)

;pl1 : power level of $1 \mathrm{H}$ hard pulse

;pl12 : power level of decoupling (10-15 kHz)

;pl13 : power level for water suppression

;spnam1 : 1H shape for 1H->13CA CP (ramp 10-20\%)

;spoal1 : N/A

;spoff1 : [ON/RES]

;sp1 : $1 \mathrm{H}$ power level during $1 \mathrm{H}->13 \mathrm{CA} \mathrm{CP}$

;spnam10 : 1H shape for $15 \mathrm{~N}->1 \mathrm{H}(\mathrm{N}) \mathrm{CP}$ (ramp 10-20\%)

;spoal10 : N/A

;spoff10 : [ON/RES]

;sp10 : $1 \mathrm{H}$ power level during $15 \mathrm{~N}->1 \mathrm{H}$ CP

;cpdprg2 : 15N decoupling pattern during acq (waltz-16)

;p17 : contact time 15N->1H(N) CP (300-500 us)

;pcpd2 : pulse length in 15N decoupling sequence (25 us)

;pl7 : power level for $15 \mathrm{~N}$ hard pulse

;pl16 : power level for $15 \mathrm{~N}$ decoupling (corr. to $10 \mathrm{kHz}$ )

;pl2 : power level for $15 \mathrm{~N}$ hard pulse

;pl20 : 15N power level for $15 \mathrm{~N}->1 \mathrm{H} \mathrm{CP}$

;spnam2 : 15N shape for 13CA->15N CP (tan-c100-w10pct)

;sp2 : 15N power level for 13CA->15N CP

;spoal2 : N/A

;spoff2 : [ON/RES]

;p15 : contact time 1H->13CA CP (5 ms)

;p16 : contact time 13CA->15N CP (10 ms)

;p18 : Q3 CO pulse duration

;p19 : Q3 CA pulse duration

;pcpd3 : pulse length in $13 \mathrm{C}$ decoupling sequence (25 us)

;cpdprg3 : 13CO/CA decoupling pattern during 15N evol (waltz-16)

;pl3 : power level of 13C hard pulse [REFERENCE]

;pl17 : power level for 13CO/CA decoupling (10 kHz)

;spnam9 : 13C shape for 13CA->15N CP (rectangle)

;sp9 : 13C power for 13CA->15N CP

;spoal9 : N/A

;spoff9 : [ON/RES CO]

;spnam18 : $13 \mathrm{CO}$ selective pulse shape (Q3)

;spoal18 : N/A 
;spoff18 : [ON/RES CO]

;spnam19 : 13CA selective pulse shape (Q3)

;spoal19 : N/A

;spoff19 : [ON/RES CA]

;spnam29 : 13C shape for $1 \mathrm{H}->13 \mathrm{CO}$ CP (rectangle)

;sp29 : 13C power level for $1 \mathrm{H}->13 \mathrm{CO} \mathrm{CP}$

;spoal29 : N/A

;spoff29 : [ON/RES CO]

;zgoptns : -Dfslg, -Dlacq, or blank

;\$COMMENT=Inverse Cp with INEPT CBCA mixing

;\$CLASS=Solids

;\$IM=3D

;\$TYPE=H detect

;\$SUBTYPE=Heteronuclear

;\$OWNER=CRMN

\#include <Avancesolids.incl>

; Start evolutions from exactly 0

"d0=0.0"

"d10=0.0"

; $1 \mathrm{H}$ settings

"spoal1=0.5" ; default value (irrelevant)

"spoff1=0.0" ; on-resonance

"spoal10=0.5" ; default value (irrelevant)

"spoff10 $=0.0 "$; on-resonance

; $15 \mathrm{~N}$ settings

"pcpd2=25" ; ;oes not work!

"plw2=plw7"

"plw16=plw2*(pow(p7/25,2))" ；15N waltz 10kHz decoupling power level

"spoal2=0.5" ; default value (irrelevant)

"spoff2=0.0" ; on-resonance

; $13 \mathrm{C}$ settings

"plw17=plw3*(pow(p3/25,2))" $\quad ; 13 \mathrm{C}$ waltz $10 \mathrm{kHz}$ decoupling power level 
"cnst22 = (sfo3-bf3)*1000000/bf3" ;CA frequency offset (ppm)

"cnst21 = cnst22+(173.7-53.7)" ；CO frequency offset (ppm)

"cnst23 = cnst22+(113.7-53.7)" ; the offset half-way CO and CA (ppm)

"p18=3.412/(95.0*bf3/1000000)" ; 95 ppm bandwidth (safe)

"spw18=plw3*pow((0.5/(p18*0.1515))/(0.25/p3),2)" ; Q3 power level

"spoal18=0.5" ; default value (irrelevant)

"spoff18=bf3*((cnst21-cnst22)/1000000)"

"p19=3.412/(105.0*bf3/1000000)" ; 95 ppm bandwidth (safe)

"spw19=plw3*pow((0.5/(p19*0.1515))/(0.25/p3),2)" ; Q3 power level

"spoal19=0.5" ; default value (irrelevant)

"spoff19=0.0" ; ; $\quad$ CA frequency

"spoal9=0.0" ; needed for offset on C

"spoff9=0.0" ; on-resonance

"spoal29=1.0" ; needed for offset on C

"spoff29=0.0" ; on-resonance CA

;"acqt $0=1 u^{\star}$ cnst11" ??

"in0=inf1"

"in10=inf2/2"

aqseq 312

1 ze

2 d1 do:f2

\#include <p15_prot.incl>

\#include <aq_prot.incl>

;1u fq=0:f3

$1 \mathrm{u} f q=c n s t 22(b f p p m): f 3$

(p1 pl1 ph3):f1

(p15:sp29 ph15):f3 (p15:sp1 ph20):f1

1u cpds1:f1

(p18:sp18 ph18):f3 ;CO selective Pi

$1 \mathrm{u}$ 


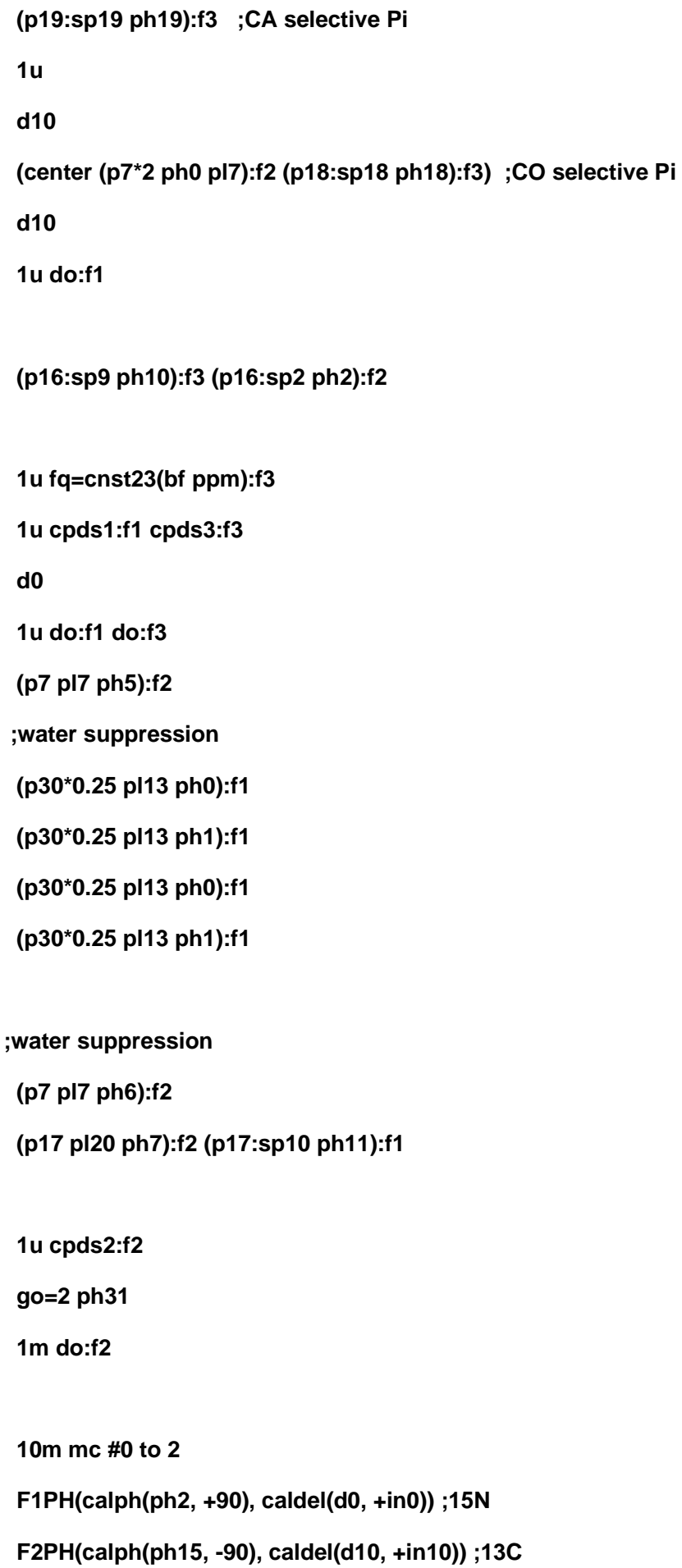




\section{Appendix}

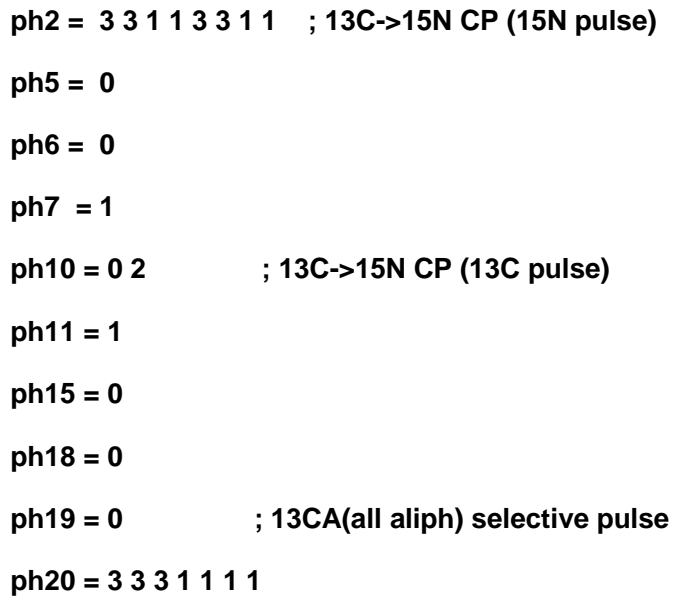

